\title{
FINITE VOLUME MODELLING OF DIFFUSIVE-VISCOUS WAVES IN A FLUID-SATURATED MEDIUM
}

\author{
by \\ VICTOR MENSAH \\ (MSc. Oil and Gas Engineering) \\ (MPhil. Geophysics) \\ (BSc. Geomatic Engineering)
}
A Thesis Submitted to the Departamento de Ingeniería Geológica y Minera, Universidad Politécnica de Madrid in partial fulfillment of the requirements for the degree of

\section{DOCTOR OF PHILOSOPHY}

Escuela Técnica Superior de Ingenieros de Minas y Energía

Supervised by

Prof. Arturo Hidalgo-Lopez

E.T.S.I. Minas y Energía

Saturday $24^{\text {th }}$ October, 2020 

Tribunal nombrado por el Sr. Rector Magfco. de la Universidad Politécnica de Madrid, el día ........ de ............... de $20 . .$.

Presidente:

Vocal:

Vocal:

Vocal:

Secretario:

Suplente:

Suplente:

Realizado el acto de defensa y lectura de la Tesis el día $\ldots \ldots \ldots$ de $\ldots \ldots \ldots \ldots \ldots \ldots$ de 20...en E.T.S.I. /Facultad......................

Calificación.

EL PRESIDENTE

LOS VOCALES

EL SECRETARIO 



\section{Resumen}

Los métodos en volúmenes finitos son técnicas bien conocidas usualmente aplicadas a la simulación de problemas basados en leyes de conservación. Presentan, además, particular interés para el tratamiento de geometrías no triviales y condiciones de contorno complejas. Además se pueden aplicar tanto a mallados no estructurales como a mallados regulares, como los que se utilizan habitualmente en el ámbito de las diferencias finitas.

Estas técnicas numéricas son además particularmente eficientes para la resolución de las ecuaciones difusivo-viscosas (DVW por sus siglas en inglés), de interés en esta tesis, dado que permiten resolver de manera muy precisa los fenómenos de propagación de ondas en un medio concreto con condiciones de contorno complejas.

En esta tesis se explora la aplicación de la aproximación por volúmenes finitos a la simulación de fenómenos basados en ondas difusivo-viscosas. Se analiza, además la estabilidad numérica en dominios tridimensionales mediante la técnica de von Neumann.

La simulación de las ondas sísmicas se realiza, en esta tesis, mediante modelos de ondas difusivo-viscosas en dos y tres dimensiones, que se resuelven mediante métodos en volúmenes finitos, en un medio fluido saturado.

Los resultados de esta simulación se comparan con los obtenidos con modelos basados en ondas acústicas, así como con modelos visco-acústicos. La simulación numérica realizada pone de manifiesto que se produce una mayor atenuación en la propagación de ondas que cuando se emplean modelos basados en ondas acústicas y en ondas visco-acústicas. También se emplea el denominado factor de calidad, $Q$, con la finalidad de obtener los Parámetros de Atenuación Activa (AAP por sus siglas en inglés), que básicamente describen la atenuación de las DVWs y se comparan con los que corresponden a las ondas visco-acústicas. 


\section{Abstract}

Finite volume methods have currently been demonstrated to be a practicable solution to the problem of complex boundary conditions over non-trivial geometries, and they also permit the application of energy methods for numerical stability analyses. Finite volume methods accommodate themselves naturally to fully unstructured grids and they can simplify to the types of grids usually employed in finite difference methods. This allows for diffusive-viscous wave (DVW) simulation models that balance the simplicity of finite difference methods for wave propagation in a medium with the detail of finite volume methods for complex wave phenomena as well as complex boundaries.

This thesis is an exploration of the finite volume approach to the simulation of the diffusiveviscous wave equation. The underlying theme in this research is to balance between numerical simulation, accuracy, and numerical stability. Second-order space and time optimised schemes in two and three spatial dimensions are developed towards the objective of discovering accurate and efficient finite volume schemes. Numerical stability is analysed in three dimensions using von Neumann's stability analyses, allowing for stable numerical scheme appropriate for three-dimensional diffusive-viscous wave modelling. Also, the accuracy of the numerical scheme is investigated for the two-dimensional and threedimensional diffusive-viscous wave models. Seismic waves are then simulated in a twodimensional and three-dimensional fluid-saturated medium and the results compared with those of the acoustic and visco-acoustic cases, respectively. The numerical results indicate stronger attenuation when compared to the acoustic and visco-acoustic case accordingly. The Quality Factor, $Q$ is used to obtain the Active Attenuation Parameters (AAP), which basically describes the attenuation of DVWs, and they are compared to those of the viscoacoustic wave.

Keywords - Numerical modelling - Seismic attenuation - Wave propagation 


\section{Dedication}

I dedicate this doctoral thesis to God Almighty and my entire Family. 
2020 by Victor Mensah 
The Book of Nature is written in the language of mathematics.

— Galileo Galilei 


\section{Declaration}

I hereby declare that this submission is my own work towards the award of Doctor of Philosophy $(\mathrm{PhD})$ degree and that, to the best of my knowledge, it contains no material previously published by another person or material which has been accepted for the award of any degree of the university, except where due acknowledgement has been made in the text.

Victor Mensah

Student Name

Signature

Date

Certified by:

Prof. Arturo Hidalgo-Lopez

Supervisor Name

Signature

Date 


\section{Related Publications}

Some of the research leading to this thesis has appeared previously in the following publications.

1. Victor Mensah, Arturo Hidalgo and Rafael Medina Ferro: Numerical modelling of the propagation of diffusive-viscous waves in a fluid-saturated reservoir using finite volume method. - Geophysical Journal International, 2019, 218, 1, 33-44.

2. Victor Mensah and Arturo Hidalgo: Modelling the effects of diffusive-viscous waves in a 3-D fluid-saturated media using two numerical methods. - Geophysical Journal International, DOI: 10.1093/gji/ggaa457. 


\section{Acknowledgements}

I would like to firstly express my deepest gratitude to God Almighty and also to my supervisor Prof. Arturo Hidalgo-Lopez, whose continuous interest, encouragement, contributions and support during my $\mathrm{PhD}$ journey I greatly appreciate. Prof. Arturo HidalgoLopez, I say thank you for your guidance throughout every phase of this research; for the knowledge shared in all aspects related to numerical methods and FORTRAN programming; as well as your great sense of humour.

I am also indebted to my former co-supervisor Prof. Rafael Medina Ferro from whom I have learned so much, and who played a vital role in the successful publication of our first article. Prof. Rafael Medina Ferro, kindly take a bow for your remarkable contributions.

My PhD journey would not have been possible without the financial support from my dearest parents, Mr. Victor Mensah and Mrs. Ethel Mensah. Dad, Mom, I say thank you for giving me the necessary tools to succeed in life and God richly bless you. The financial support is greatly acknowledged.

I am grateful to my lovely and supportive brothers for all their sacrifices and support throughout these years. I ask the Almighty God to make you my siblings in the next life. God richly bless you all.

Lastly, I wish to deeply thank and dedicate this research to my pretty wife, Wilhelmina, my adorable kids, Ethel, Victor, Jason, and Jaden, for all their sacrifices and support. My darling Wilhelmina, I love you and forever will. My adorable angels, I love you all and forever remain indebted to you for your sacrifices. 


\title{
Table of Contents
}

\author{
Resumen
}

Abstract

Dedication

Declaration

Related Publications

Acknowledgements

$\begin{array}{lll}\text { Contents } & \mathbf{v}\end{array}$

List of Figures $\quad$ ix

$\begin{array}{ll}\text { List of Tables } & \mathbf{x} \\ \end{array}$

List of Abbreviations $\quad$ xi

List of Symbols $\quad$ xiii

1 Introduction 1

1.1 Research Motivation . . . . . . . . . . . . . . . . 1

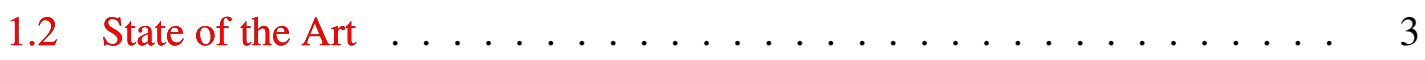

1.3 Practical Application of the Diffusive-Viscous Theory . . . . . . . . . 8

1.4 Research Objectives . . . . . . . . . . . . . . . . . . . . . 9

1.5 Thesis Outline . . . . . . . . . . . . . . . . 10 
2.1 The diffusive-viscous theory . . . . . . . . . . . . . . . . 12

2.1.1 The diffusive-viscous wave equation . . . . . . . . . . 13

2.1.2 The derivation and discussion of the diffusive-viscous wave equations ................................. 14

2.1.3 The propagating wavenumber and attenuation coefficient of the diffusive-viscous wave . . . . . . . . . . . . . 16

2.1.4 The Quality Factor, $Q$ and Active Attenuation Properties (AAP), $\zeta$ and $\eta \ldots \ldots \ldots \ldots \ldots \ldots$

2.1.5 Source implementation . . . . . . . . . . . . . . . 21

2.1.6 Boundary conditions . . . . . . . . . . . . 23

2.1.7 Seismic impulse responses . . . . . . . . . . . . . 25

2.2 Computational methods for diffusive-viscous waves in the time domain . 27

2.2.1 Geometric methods . . . . . . . . . . . . . . . 27

2.2.1.1 Ray-tracing method . . . . . . . . . . 28

2.2.1.2 Image-source approach . . . . . . . . . . . . 29

2.2.2 Finite-difference method . . . . . . . . . . . . . 30

2.2.2.1 Computational costs for the wave equation with finite difference methods ............ 31

2.2.3 Finite-element and finite-volume methods . . . . . . . . . . . 33

2.2.3.1 Finite element methods . . . . . . . . . . . 34

2.2.3.2 Finite volume methods . . . . . . . . . . 34

2.2.3.3 Standard cell-centred method . . . . . . . . . . 34

2.2.3.4 Staggered-grid methods . . . . . . . . . . . 36

2.2.3.5 Vertex-centred method . . . . . . . . . . 37

2.2.3.6 Godunov-type methods . . . . . . . . . . . . 38

2.2.3.7 Finite element and finite volume methods for the wave equation: computational costs . . . . . . . . . . 40

2.2.4 Boundary element methods . . . . . . . . . . . . . . . 40

2.3 Summary .............................. 41 
3 Methodology $\quad 42$

3.1 Finite volume method . . . . . . . . . . . . . . . . . 42

3.1 .1 Discretization . . . . . . . . . . . . . . 46

3.2 Integration . . . . . . . . . . . . . . . 48

3.2.1 Midpoint rule . . . . . . . . . . . . . . . . 49

3.2 .2 Gaussian quadrature . . . . . . . . . . . . . 50 50

3.3 Interpolation . . . . . . . . . . . . . . 51

3.3 .1 Function reconstruction $\ldots \ldots \ldots 52$

3.4 Reconstruction from cell averages. General ideas . . . . . . . . . . . . 53

3.4.0.1 Piecewise constant reconstruction . . . . . . . . . 56

3.4.0.2 Piecewise linear reconstruction . . . . . . . . 57

3.4.0.3 Piecewise parabolic reconstruction . . . . . . . 59

3.4 .1 Shape functions . . . . . . . . . . . . . . 60

3.4.2 Assuring flux consistency ............... 61

3.5 Time integration . . . . . . . . . . . . . . 62

3.5.1 Runge-Kutta TVD time integration . . . . . . . . . . . 63

3.6 Summary . . . . . . . . . . . . . . . . . . . . . 64

4 Two-dimensional considerations $\quad \mathbf{6 5}$

4.1 Meshing .............................. 65

4.2 Designing control volumes . . . . . . . . . . . . . . 66

4.2.1 Sub-control volumes $(\mathrm{SCV}) \ldots \ldots . \ldots 68$

4.3 Conventions .......................... 70

4.4 Integration . . . . . . . . . . . . . . 72

4.4.1 Midpoint rule . . . . . . . . . . . . . . 73

4.4 .2 Gaussian quadrature . . . . . . . . . . . . . 74

4.5 Interpolation method $\ldots \ldots \ldots \ldots$. . . . . . . . . 81

4.5.1 Piecewise constant reconstruction . . . . . . . . . . 81

4.5.2 Piecewise linear reconstruction . . . . . . . . . . . . . 81

4.5.3 Shape functions . . . . . . . . . . . . . . 83

4.6 Complete finite volume discretization . . . . . . . . . . . . . 84 
4.7 Summary $\ldots \ldots \ldots \ldots \ldots \ldots \ldots \ldots$

5 Diffusive-viscous wave modelling in two-dimensions with the simple Carte$\begin{array}{ll}\text { sian scheme } & \mathbf{8 6}\end{array}$

$5.1 \quad$ Numerical Model . . . . . . . . . . . . . . . . . . . . . 86

5.1.1 Meshing and function reconstruction . . . . . . . . . . 87

5.1.2 Flux approximations and time integration . . . . . . . . . . 89

5.2 Error norm computation . . . . . . . . . . . . . . 91

5.3 Accuracy of the numerical scheme . . . . . . . . . . . . . . 93

5.4 Numerical Results . . . . . . . . . . . . . . . . . . . . . . . . . . . . . . 94

5.4.1 Source and receiver implementation . . . . . . . . . . . . 96

5.5 Discussion . . . . . . . . . . . . . . . . . . . . . 99

5.6 Conclusion . . . . . . . . . . . . . . . . 101

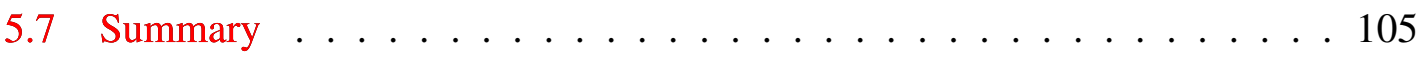

6 Three-dimensional considerations $\quad 106$

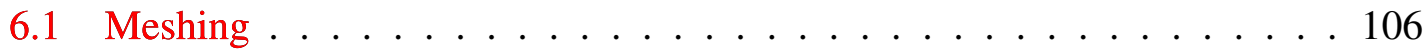

6.2 Designing control volumes . . . . . . . . . . . . . . 107

6.3 Conventions . . . . . . . . . . . . . . . . . . . 109

6.4 Integration . . . . . . . . . . . . . . . . . 110

6.4.1 Midpoint rule (one-point Gaussian quadrature) . . . . . . . . 111

6.4 .2 Gaussian quadrature . . . . . . . . . . . . . . 112

6.5 Interpolation . . . . . . . . . . . . . . . . 115

6.5.1 Piecewise constant reconstruction . . . . . . . . . . 115

6.5.2 Piecewise linear reconstruction . . . . . . . . . . . 115

6.5.3 Shape function . . . . . . . . . . . . . 117

6.6 Complete finite volume discretization . . . . . . . . . . . . . 117

6.7 Summary . . . . . . . . . . . . . . . . . . 118

7 Diffusive-viscous wave modelling in three-dimensions with the simple Carte$\begin{array}{lr}\text { sian scheme } & 119\end{array}$

$7.1 \quad$ Numerical Model . . . . . . . . . . . . . . . . . . . . . . . . . . . 119 
7.2 Validation of the numerical scheme . . . . . . . . . . . . . . 124

7.3 Von Neumann Stability Analysis . . . . . . . . . . . . . . . . 128

7.4 Numerical Results . . . . . . . . . . . . . . . . . . . . 133

7.5 Discussion . . . . . . . . . . . . . . . . . 139

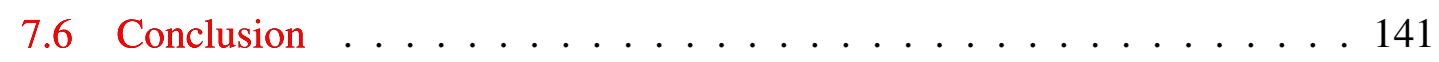

7.7 Summary . . . . . . . . . . . . . . . . . . . 142

8 Conclusions and final remarks $\quad 143$

8.1 Summary . . . . . . . . . . . . . . . . . . . 143

8.2 Recommendations for future research . . . . . . . . . . . . . . 144

$\begin{array}{ll}\text { References } & 146\end{array}$ 


\section{List of Figures}

2.1 The Ricker wavelet $s(t)$, and the frequency spectrum, $\Re(\omega)$, with the dominant angular frequency of $60 \pi \mathrm{rad} / \mathrm{s} \ldots \ldots . \ldots 23$

2.2 Description of a domain as a time-domain filter . . . . . . . . . . 26

2.3 Description of the method to acquire the impulse response . . . . . . . 26

3.1 Finite volume method showing (a) cell-centered and (b) vertex-centered control volumes. . . . . . . . . . . . . . . . . . . 43

3.2 Control volume of the vertex-centred finite volume method (a). 2-D triangle cell (b). 3-D tetrahedron cell . . . . . . . . . . . . . . . . . . 44

3.3 The "two-point" quadrature methods in (a) 1-D; (b) 2-D; (c) 3-D . . . . . 50

3.4 Discretization of a one-dimensional domain into computational cells(grid) of width, $\delta x$. The cell centers are illustrated by dark-filled circles and the vertical lines indicate the cell edges; the cell number is illustrated above the cells while the cell edges are indicated by the numbers below. . . . . . 56

3.5 Piecewise Constant Approximation. . . . . . . . . . . . . . . 56

3.6 Piecewise Linear Approximation (Red lines). The green-dashed lines are cell averages given by a piecewise constant function. . . . . . . . . . 58

3.7 Piecewise Parabolic Approximation (Purple lines). The green-dashed lines are cell averages given by a piecewise constant function.. . . . . . . 59

3.8 Computing fluxes using grid-wise interpolation. Red lines represent the control volume faces. . . . . . . . . . . . . . . . . . 63

4.1 Finite volume meshes: (a) structured; (b) unstructured. . . . . . . . . . . 66

4.2 Designing control volumes: (a) cell-centered control volume; (b) vertexcentered control volume. . . . . . . . . . . . . . . 67 
4.3 Correct, anticlockwise ordering. . . . . . . . . . . . . . 68

4.4 A sub-control volume (a) as one of four comprising a control volume; (b) on its own. . . . . . . . . . . . . . . . . . 69

4.5 A sub-control volume (a) with a control volume; (b) on its own. . . . . . 70

4.6 Numbering convention for nodes and control volume faces. . . . . . . . . 71

4.7 Outer normal for a boundary element: (a) cell-centered boundary element; (b) vertex-centered boundary element . . . . . . . . . . . . . . 72

4.8 Line integral tracks for control volume methods: (a) cell-centered; (b) vertex-centered. .................. 73

4.9 Gaussian quadrature points in a control volume: (a) four-point Gaussian quadrature; (b) nine-point Gaussian quadrature. . . . . . . . . . . . . . 76

4.10 Accuracy of line integral methods. . . . . . . . . . . . . . . . . . . 80

4.11 Evolution of fluxes in time at control volume faces. . . . . . . . . . . . . 82

4.12 Piecewise Linear Reconstruction method applied to variable values, $u$ and $v$, adopted from Mensah, Hidalgo, and Ferro (2019). . . . . . . . . . . 83

5.1 The Discretisation of a two-dimensional domain into Cartesian finite volumes adopted from Mensah et al. (2019). . . . . . . . . . . . . . . . 88

5.2 The fluid-saturated model with an $80 \mathrm{~m}$ thick (1)oil-, (2)water- and (3)gassaturated layer embedded in a homogeneous half space, adopted from Mensah et al. (2019). . . . . . . . . . . . . . . . . . . . . . 9

5.3 Comparison of the exact solution (5.16) of the problem (5.15) with the numerical solutions obtained on three different meshes for output time $t=0.25$ cutting the surface with the plane $\mathrm{x}=0.5$. Top: Displacement. Bottom: Velocity. Adopted from Mensah et al. (2019). . . . . . . . . . . 95

5.4 Snapshots of diffusive-viscous wavefields (a), (b), (c) and acoustic wavefields (d), (e), (f) in a lateral heterogeneous medium for Particle Displacement (notice varying color scaling), adopted from Mensah et al. (2019).

5.5 Snapshots of diffusive-viscous wavefields (a), (b), (c) and acoustic wavefields (d), (e), (f) in a lateral heterogeneous medium for Particle Velocity (notice varying color scaling), adopted from Mensah et al. (2019). . . . . 98 
5.6 Seismograms generated from Particle motions (Displacement and Velocity) for diffusive-viscous wave (continuous line) compared with acoustic wave (dashed-line) for three receivers placed on the surface of the model with multiple reflections indicated, adopted from Mensah et al. (2019). . . 102

5.7 Seismograms generated from Particle Displacement for diffusive-viscous wave (continuous line) compared with acoustic wave (dashed-line) for the set receivers with multiple reflections indicated, adopted from Mensah et al. (2019) . . . . . . . . . . . . . . . . . . 103

5.8 Seismograms generated from Particle Velocity for diffusive-viscous wave (continuous line) compared with acoustic wave (dashed-line) for the set receivers with multiple reflections indicated, adopted from Mensah et al. (2019). . . . . . . . . . . . . . . . . . . 104

6.1 A three-dimensional sub-control volume within a control volume. . . . . 108

6.2 Three-dimensional control volumes. . . . . . . . . . . . . . . . . 108

6.3 Notation convention for nodes, faces, and sub-control volumes. . . . . . . 109

6.4 A single hexahedral control volumes. . . . . . . . . . . . . . . 111

7.1 The discretisation of a 3-D domain into Cartesian finite volumes. . . . . . 121

7.2 The fluid-saturated model with an $80 \mathrm{~m}$ thick (1)oil-, (2)water- and (3)gassaturated layer embedded in a homogeneous half space. . . . . . . . . . . 123

7.3 Representation of the displacement, $u(x, 0, z, 0.1)$. Cut with the plane

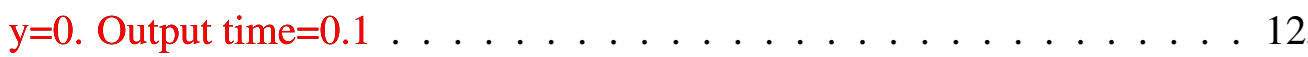

7.4 Comparison of the numerical solution of the problem Eq.(7.8) for the displacement $u(0,0, z, 0.1)$ with the exact solution given by Eq.(7.10) for different meshes. Cut with the planes $\mathrm{x}=0 ., \mathrm{y}=0 . \ldots \ldots 12$

7.5 Comparison of the numerical solution of the problem equation (7.8) for the velocity $v(0,0, z, 0.1)$ with the exact solution given by equation (7.10) for different meshes. Cut with the planes $\mathrm{x}=0 ., \mathrm{y}=0 . \ldots \ldots 127$ 
7.6 Region in the plane $\mu-\alpha$ where the spectral radius satisfy $\rho(\mathbf{A}) \leq 1$. The stability region is also identified. The results have been obtained using $\beta=0$. (top frame) and $\beta=1.5$ (bottom frame) . . . . . . . . . . . . 134

7.7 Numerical solution of the problem Eq.(7.25) using $\alpha=0.1, \mu=0.1$, $\beta=1.5$, output time $t_{\max }=60.0$. The plot is a cut with the plane $y=5.0 .135$

7.8 Snapshots of diffusive-viscous wave-fields (a), (b) and visco-acoustic wavefields (c), (d) in a 3-D fluid-saturated medium for Particle Displacement (notice varying color scaling) . . . . . . . . . . . . . 137

7.9 Snapshots of diffusive-viscous wave-fields (a), (b) and visco-acoustic wavefields (c), (d) in a 3-D fluid-saturated medium for Particle Velocity (notice varying color scaling) . . . . . . . . . . . . . . . . 138

7.10 Seismograms generated from Particle Displacement and Particle Velocity for diffusive-viscous wave (solid line) compared with visco-acoustic wave (dashed-line) for three receivers (A, B and C) placed on the surface of the model with multiple reflections indicated . . . . . . . . . . . . . . . 140 


\section{List of Tables}

5.1 Convergence rates for problem (Eq. 5.15). Using $\zeta=65.4 \mathrm{~Hz}, \eta=$ $0.0147 \mathrm{~m}^{2} / \mathrm{s}$ and $\nu=10 \mathrm{~m} / \mathrm{s}$. Output time $t=0.25$. Adopted from Mensah et al. (2019). . . . . . . . . . . . . . . . . . 94

5.2 Physical Parameters of the Model, adopted from Mensah et al. (2019) . 96

7.1 $L_{2}$ norm of the error and order of accuracy for the displacement (unknown

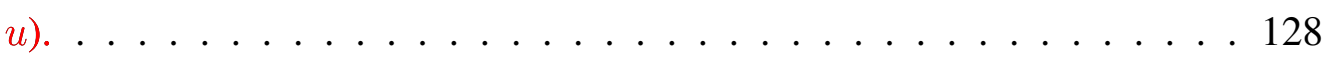

7.2 $L_{2}$ norm of the error and order of accuracy for the velocity (unknown $v$ ). . 128 


\section{List of Abbreviations}

FVM ........ Finite Volume Method

FV .......... Finite Volume

DVWE ....... Diffusive-Viscous Wave Equation

1-D .......... One-Dimension

2-D ......... Two-Dimensions

3-D .......... Three-Dimensions

PDEs ........ Partial Differential Equations

ODEs $\ldots . \ldots$. Ordinary Differential Equations

FDM ......... Finite Difference Method

FEM $\ldots . . \ldots \ldots$ Finite Element Method

SEM $\ldots . . \ldots$.... Spectral Element Method

BEM ......... Boundary Element Method

LFS ......... Low Frequency Shadows

ADER-DGM .. Arbitrary High-Order Derivatives Discontinuous Galerkin Method TVD ........ Total Variation Diminishing

AAP $\ldots \ldots \ldots$ Active Attenuation Properties

ABC $\ldots \ldots \ldots$ Absorbing Boundary Condition

SIR ........ Seismic Impulse Response

GA $\ldots \ldots \ldots$ Geometrical Acoustics 
CFL ........ Courant-Friedrichs-Lewy

KIE ........ Kirchhoff Integral Equation

KHIE ....... Kirchhoff-Helmholtz Integral Equation

CV ......... Control Volume

LHS ......... Left Hand Side

RHS ........ Right Hand Side

CV-FEM ..... Control Volume Finite Element Method

RK ........ Runge-Kutta

SCV ......... Sub Control Volume

MUSCL ....... Monotonic Upstream-Centered Scheme for Conservation Laws

AVO $\ldots \ldots \ldots$ Amplitude Versus Offset

AVA .......... Amplitude Versus Angle

RK2 ........ Second order Runge-Kutta 


\section{List of Symbols}

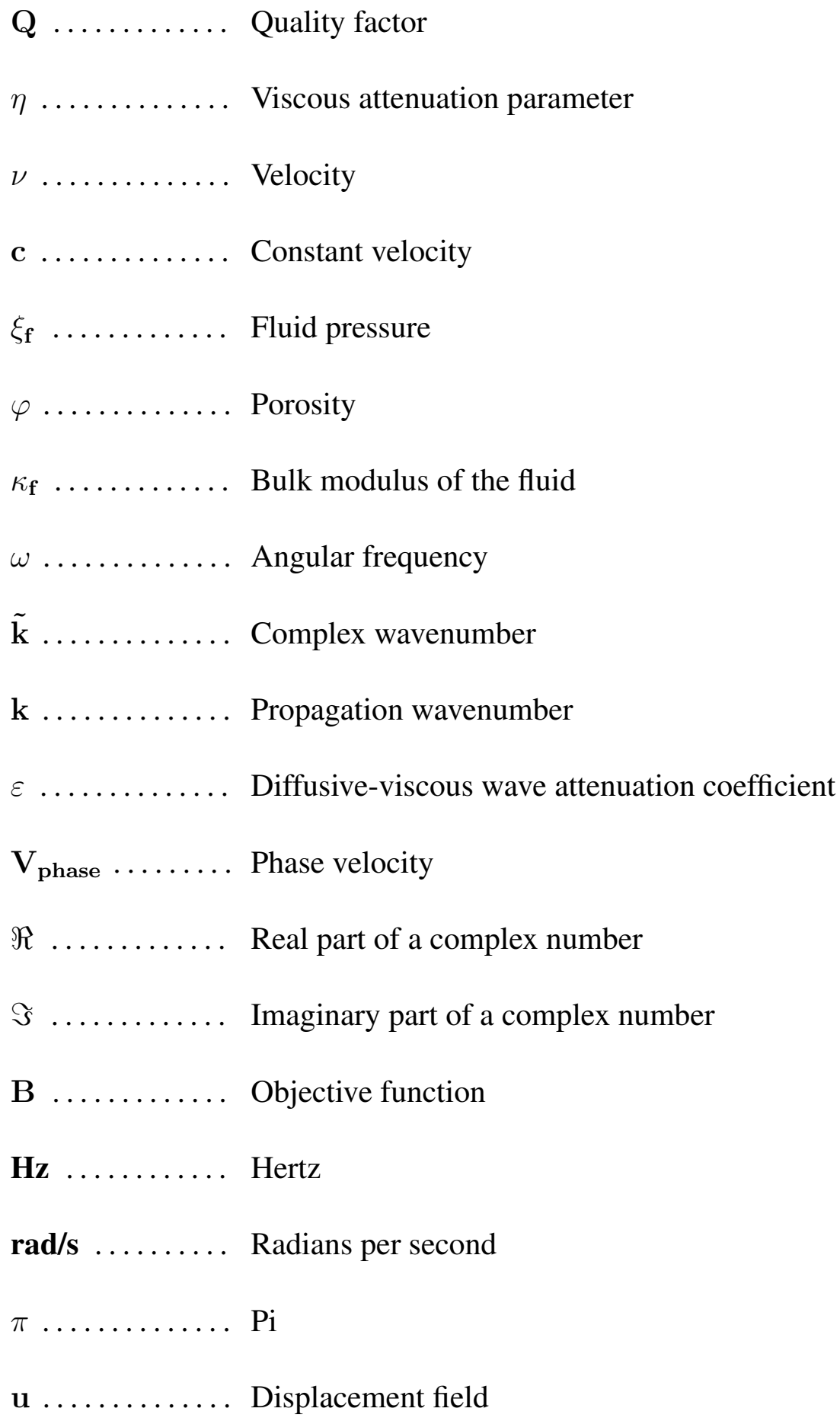


$\mathbf{v} \ldots \ldots \ldots \ldots$ Velocity field

f $\ldots \ldots \ldots \ldots$ Frequency

$Q_{j} \ldots \ldots \ldots$ shape function

$\min \ldots \ldots \ldots . \quad$ Minimum 


\section{Chapter 1}

\section{Introduction}

\subsection{Research Motivation}

Interest in extensive research of numerical seismology, focused on the development of methods to approximate the propagation of seismic waves in the Earth has increased over the last four decades. The motivation of this is that numerical simulation based on seismic wave equations is an efficient approach to investigate the seismic response of a reservoir. Numerical simulation also tends to be the foundation of seismic data interpretation including migration and inversion. In order to simulate wave propagation and implement numerical simulation methods in practical seismic exploration, the wave equations should be selected suitably since exact analytical solutions are non-existent for subsurface models of interest in exploration and global seismology. Numerical methods possess certain advantages which are that they are based on seismic wave equations (acoustic, elastic, visco-acoustic, visco-elastic, Biots, diffusive-viscous, etc.) for different kinds of media (homogeneous, heterogeneous, fluid-filled, etc.). Therefore, direct waves, primary and multiple reflected and transmitted waves, surface waves, diffracted waves, converted waves, and critically diffracted waves can be simulated within the physical model whenever present.

Real subsurface structures are complex and often multi-phase media due to fluid saturation, as a result, the frequently applied models such as acoustic, elastic media, among others, are unable to characterize the information of real subsurface structures. The anelastic attenuation is know to occur when the wave propagate in fluid saturated media. 
The relationship between the frequency-dependent reflections and fluid saturation in a reservoir is quite complex, but there is a direct connection between the character of porous layer saturation and seismic response.

The wave propagation problem is computationally and mathematically challenging. The reason is due to the complexity and scale of the geophysical models in exploration and global seismology. A cross-disciplinary approach involving geophysics, applied mathematics and large-scale computing is required to effectively deal with this problem.

In recent years, the Finite-Volume Methods (FVM) has gained popularity in the field of seismic numerical wave propagation. The FVM has some fascinating advantages, among which are the flexibility with which they deal with surface topography. They also have that versatility when dealing with discontinuities in the subsurface model, boundary conditions and not limited to media parameter but also wavefields (velocity, displacement, stress, pressure, depending on the formulation). They even can approximate the wavefield with arbitrarily high order polynomials. An intriguing advantage of the FVM is also that they obey the law of conservation of energy. They can deal with more general meshes, and they possess that suitability for local time stepping and parallel implementations.

Numerical stability analysis and the grid dispersion criteria are the primary tools for determining the applicability of a numerical method to the seismic wave propagation problem. For the numerical solution to remain bounded, the stability analysis is required to determine the largest time-step for the time-marching scheme. The grid dispersion criterion, on the other hand, is responsible for determining the largest sampling ratio for the spatial discretisation such that the numerical solution converges to an acceptable accuracy.

This dissertation aims to implement FVM for the diffusive-viscous wave propagation and to investigate its stability. In addition, we will compute wavefields (velocity and displacement) on realistic geophysical models with this method. We initially derive a FV scheme for the diffusive and viscous wave equation (DVWE) and then simulate the propagation of waves based on the diffusive and viscous wave theory in a fluid-saturated media using FV methods. To the best of our knowledge, this is the first time FV methods have been applied to the DVWE. We compare our results with those from Zhao, Gao, and Zhao 
(2014) and the numerical results show that waves clearly attenuate as they propagate into the oil-, water-, and gas-saturated layers when compared with the acoustic scenario. We extend the 2-D diffusive-viscous wave equation presented in He, Xiong, and Bian (2008) and Mensah et al. (2019) to 3-D. We also estimate the active attenuation parameters in the diffusive-viscous wave equation, as an attempt to further appreciate the properties of diffusive-viscous waves, using the Quality factor, $Q$ approach in the visco-acoustic medium. We then extend the FV scheme developed by Mensah et al. (2019) to 3-D. We finally synthesise the seismograms of diffusive-viscous waves in a fluid-saturated media and make comparisons of the results from the diffusive-viscous waves with those from the visco-acoustic waves in the time domain.

\subsection{State of the Art}

Seismic wave propagation problem, in the context of numerical simulations of seismic events, is realized by solving numerically, the partial differential equations (PDEs) of elastic wave propagation. Finite Difference methods (FDM), Finite Element methods (FEM), Finite Volume methods (FVM) and Spectral Element methods (SEM) are methods that can be used to numerically approximate PDEs over the spatial domain (Agreste \& Ricciardello, 2011). The ability to compute synthetic seismogram has always been very crucial in geophysics since accurate forward modeling can be worthwhile in seismic interpretation and inversion. An improved forward modeling is therefore a very beneficial tool for seismic interpretation and also an initial step to inverse problems.

Finite-difference (FD) methods have proved their efficiency and usefulness in solving and modelling seismic wave propagation in complex media (Kelly, Ward, Treitel, \& Alford, 1976), and have been successfully applied to numerous physical problems over the past decades. However, there are certain significant issues fundamental to the underlying Cartesian grid which the FD methods encounters, such as parasite reflections in the case of boundaries with complex topography. Computation power is consistently on the rise, and this has enabled the development of other methods, which focus a lot on this same interest. However, the fact that they are implemented using regular grids gives rise to sev- 
eral numerical problems, including numerical anisotropy related to the anisotropy of the grid. A more general alternative is the Finite Volume scheme since it may deal directly with irregular grids. The finite-volume (FV) method (Dormy \& Tarantola, 1995) and the Discontinuous Galerkin method (Dumbser \& Käser, 2006) have played a significant role in simulating 3D elastic seismic wave equation. Meshes fitting quite complex topographies can be implemented with the aforementioned methods, thus reducing the staircase pattern related to FD methods. The Finite Volume Method (FVM) was introduced into the field of computational fluid dynamics in the beginning of the seventies (McDonald, 1971; MacCormack \& Paullay, 1972). In Hermeline (1993), the FVM was implemented in dealing with a dynamical problem concerned with Maxwell's equations. In Dormy and Tarantola (1995), the FVM was applied as a general approach for numerical estimation of derivatives when they studied elastic wave propagation. Several authors have as well investigated the acoustic problems using finite volume methods.

There has, currently, been an increasing agreement on the successful application of lowfrequency seismic signals to accurately map out hydrocarbon reservoirs. According to Brown (2009), Chapman, Chesnokov, Sandhya Devi, and Grechka (2009), and Geertsma and Smit (1961), seismic reflection properties have, in most cases, been discovered to be frequency-dependent. Various geologic environments and data-sets reveal this frequency dependence of seismic reflections from the fluid-saturated porous media, and many recent laboratory and field examples as well, reveal the potential advantages of seismic lowfrequency anomalies in hydrocarbon discovery. In Taner, Koehler, and Sheriff (1979) it was once indicated, whilst analyzing complex seismic traces, that low-frequency seismic (LFS) signals often occurred in reflections immediately beneath oil layers, gas sands, condensate layers and fractured zones in tight strata. In Ebrom (2004), ten probable factors likely to generate low-frequency shadows (LFS) were outlined and they intended to precisely apply LFS in the identification and delineation of gas zones and to distinguish commercial gas-bearing reservoirs from those that are non-commercial. Their findings are, nonetheless, empirical with unexplained physical mechanisms (e.g., Chapman, Liu, \& Li, 2006; V. A. Korneev, Goloshubin, Daley, \& Silin, 2004; Tai, Puryear, \& Castagna, 2009; Tisato \& Madonna, 2012) even though LFS signals are important indicators of 
hydrocarbon reservoirs.

The revolutionary developments in computing technology have made the fundamental but yet tricky modelling of seismic wave propagation in a complex three-dimensional(3D) media nowadays achievable by numerical computations. Modelling the propagation of waves numerically, in the context of seismic exploration, has become undeniably a critical research area, primarily as a result of the increasingly geological complexity of regions in which seismic acquisition experiments are carried out. The advancement of seismology and its computational hardware has led to the development of many methods. These include the finite difference method (Käser, Igel, Sambridge, \& Braun, 2001), the finite element method (Aagaard, Hall, \& Heaton, 2001; Bao et al., 1998; H. Zhang et al., 2007), the finite volume method (e.g., Benjemaa, Glinsky-Olivier, Cruz-Atienza, Virieux, \& Piperno, 2007; Brossier, Virieux, \& Operto, 2008), the spectral element method (e.g., Chaljub et al., 2007; Cohen, 2013; Faccioli, Maggio, Paolucci, \& Quarteroni, 1997; Komatitsch \& Vilotte, 1998; Komatitsch \& Tromp, 1999), the discontinuous Galerkin Method (e.g., Etienne, Chaljub, Virieux, \& Glinsky, 2010) and the arbitrary high-order derivatives discontinuous-Galerkin method (ADER-DG) (e.g., de la Puente, Käser, Dumbser, \& Igel, 2007; de la Puente, Dumbser, Käser, \& Igel, 2008; de la Puente, Ampuero, \& Käser, 2009; Dumbser \& Käser, 2006; Käser \& Dumbser, 2006). The discontinuous Galerkin Method is a high-order finite element method that employs orthogonal Chebyshev or Legendre polynomial as the basis function. The ADER-DG also falls under the class of high-order finite element method but makes use of numerical fluxes rather than the basis functions to link elements. More often than not, the finite difference method is employed to achieve this objective (Etgen, 2007; Virieux, 1986).

Igel, Nissen-Meyer, and Jahnke (2002) applied a high-order staggered-grid FD scheme to the elastic wave equation in spherical coordinates. They indicate that the physical space is limited to spherical sections which do not include the axis $\theta=0$ when using regular grid spacing in a single domain. Accurate modelling of 3-D wave propagation is essential to understand details in the seismic wavefield which are observed on regional and global scales on the Earth's surface. The 3-D technique will permit to study the wavefield due to laterally heterogeneous structures, such as subduction zones, plumes or oceanic ridges 
(Igel et al., 2002). Even though ray-theory based methods offer the reconstruction of the long-wavelength structures describing predominantly phase effects, it is interesting to be able to study frequency-dependent waveform effects of 3-D structures due to scattering. Stephen (1983) proposed a finite-difference (FD) scheme that systematically specifies the problem in terms of displacements in both elastic and acoustic regions. His FD scheme also considers that the elastic parameters are constant within a spatial interval on both sides of the flat fluid-solid interface and defines as well an imaginary elastic boundary immediately above the fluid-solid interface.

Additionally, there is the need for an initial solution on this imaginary elastic boundary before solving for the displacement fields on the interface mentioned above, which results in additional computational cost. Both the spectral-element and finite-element methods deal with the velocity potential rather than the pressure in the fluid regions. As a result, both methods assume a homogeneous density throughout the fluid. Even though applying the velocity potential in the spectral-element approach results in a symmetric discrete system, the coupling between elements located on both sides of the fluid-solid interface produces a non-diagonal matrix. Komatitsch, Barnes, and Tromp (2000) indicate that the introduction of a staggered iterative scheme into the spectral-element system helps avoid the matrix inversion, which as well results in additional computational cost. The finiteelement approach tends to be computationally expensive and requires large amounts of computer memory in modelling the propagation of seismic waves. Both the finite-element and spectral-element methods have the advantage of dealing with a distorted fluid-solid interface by implementing curved or irregular elements. Komatitsch and Tromp (1999), indicate that the spectral-element process requires extra nodes to describe the fluid-solid interface topography correctly.

The high computational efficiency and good adaptability of the finite-volume method (FVM) to complex geometry make it an efficient alternative tool for modelling wave propagation. The Finite Volume Method (FVM) was initially introduced into the field of computational fluid dynamics in the beginning of the seventies (McDonald, 1971; MacCormack \& Paullay, 1972). The FVM was also used as a general approach for the numerical estimation of derivatives by (Dormy \& Tarantola, 1995) when they investigated 
elastic wave propagation. Oil and gas exploration development requires a better description of underground structures. It is for this and many other reasons that many methods have developed to improve the accuracy or stability of FVM (e.g., Dumbser \& Käser, 2006).

At low seismic frequency, laboratory analysis presents vital authentication of attenuation and dispersion of the frequency (Batzle, Han, \& Hofmann, 2006; V. A. Korneev et al., 2004; Tisato \& Madonna, 2012). An attempt was made by Y. Liu (2004) to numerically explain LFS in seismic reflection and they applied an alternating fluid/solid frame layered model. In Goloshubin and Korneev (2000), Goloshubin, Korneev, and Vingalov (2002) and, V. A. Korneev et al. (2004), the seismic response of fluid-saturated porous media by physical model experiments and field data was investigated. They indicate from their laboratory analysis that reflections emanating from a fluid-saturated layer possess rather higher amplitude and delayed travel time at low-frequencies, in addition to loss of high-frequency energy. They introduced a new 1D scalar wave-propagation equation which considers the diffusive and viscous attenuation parameters and also explains the frequency-dependent reflection effects in the porous layer. Nevertheless, the numerical implementation of the equation was not further investigated. Attempts by certain models based on Biot's theory (Goloshubin \& Bakulin, 1998; Biot, 1956, 1962; Dvorkin, Mavko, \& Nur, 1995), squirt flow, the reflection properties of an elastic layer (V. A. Korneev et al., 2004) and patchy saturation models (Mavko \& Mukerji, 1998) to justify the characteristics of low-frequency effects were defeated due to the limit of low-frequencies. Numerical simulations of LFS based on the diffusive and viscous wave equation were performed in a work by Xiong, He, and Huang (2008), and pragmatic suggestions to theories and procedures for identifying the physical mechanism of LFS were outlined.

The physical phenomenon governing low-frequency seismic anomalies related to hydrocarbon reservoirs has not yet been thoroughly understood. Phase shifts and energy redistribution amidst various frequencies was observed by Goloshubin, Verkhovsky, and Kaurov (1996); Goloshubin and Bakulin (1998), by comparing water- and gas-saturated rocks. According to discoveries made by V. A. Korneev et al. (2004), reflections generated from a fluid-saturated layer possess an increased amplitude and delayed travel-time 
at low frequencies when compared to observations from a dry rock in both laboratory and field data. The diffusive-viscous wave equation was proposed by empirical methods to describe the frequency-dependent phenomena. To achieve this, the scalar-wave equation was modified by including a diffusive dissipation and a viscous damping term, which was further applied to investigate the characteristics of dispersion and attenuation of waves in fluid-saturated media (Goloshubin \& Korneev, 2000; He et al., 2008; V. A. Korneev et al., 2004). Nonetheless, obtaining the attenuation parameters in the diffusive-viscous wave equation is strenuous, if not impossible. Therefore Zhao, Gao, and Liu (2014) investigated the variations of frequency-dependent reflection coefficient concerning incident angles based on the diffusive-viscous wave equation. Zhao, Gao, and Zhao (2014) further studied the propagation of diffusive-viscous waves using flux-corrected transport FDM. It is therefore important to study the propagation properties of the diffusive and viscous waves since this theory can also be used to identify oil and gas reservoirs by way of isolating the LFS anomaly from seismic data.

Furthermore, Mensah et al. (2019) studied the propagation of diffusive-viscous waves in a fluid-saturated media using FVM. In their work, they derive a finite volume scheme for the 2-D diffusive-viscous wave equation. They then simulate the propagation of waves based on the diffusive and viscous wave theory in a fluid-saturated medium using their proposed FV scheme. They tested the accuracy of their numerical scheme, and their scheme proves to be stable even for a time step larger than that given in Zhao, Gao, and Zhao (2014) for the higher-order finite difference scheme.

\subsection{Practical Application of the Diffusive-Viscous Theory}

Seismic wave numerical modeling plays a vital role in exploration seismology. It is a useful tool for seismic interpretation and works as the initial step to the inversion process. Another important application of seismic modelling is the evaluation and design of seismic surveys.

The diffusive-viscous theory can be used to identify oil and gas reservoirs through abstracting the low-frequency shadow phenomenon from seismic data. Therefore, it is es- 
sential to investigate the propagation properties of the diffusive-viscous waves.

\subsection{Research Objectives}

The objective of this research is to develop an efficient framework that permits for the accurate and numerically stable modelling of the diffusive-viscous wave behaviour in a fluid-saturated media, while incorporating the following details:

- Derive a FV scheme for the diffusive-viscous wave equation (DVWE) and then simulate the propagation of waves based on the diffusive-viscous wave theory in a fluid-saturated medium.

- Synthesise the seismograms of diffusive-viscous waves in a 2-D fluid-saturated medium and make comparisons of the results from the diffusive-viscous waves with those from the acoustic waves in the time domain.

- Extend the 2-D diffusive-viscous wave equation presented in $\mathrm{He}$ et al. (2008); Mensah et al. (2019) to 3-D and derive a finite volume scheme for the diffusiveviscous wave equation (DVWE) in 3-D.

- Estimate the active attenuation parameters in the diffusive-viscous wave equation, as an attempt to further appreciate the properties of diffusive-viscous waves, using the Quality factor Q approach in the visco-acoustic medium.

- Synthesise the seismograms of diffusive-viscous waves in a 3-D fluid-saturated medium and make comparisons of the results from the diffusive-viscous waves with those from the visco-acoustic waves in the time domain.

To achieve these objectives, the finite volume method (FVM) will be mainly employed, operating over structured grids to cover the interior of the domain, which is principally governed, by the diffusive-viscous wave equation. An absorbing type boundary condition will be employed to address concerns at the boundaries. Von Neumann stability analysis will also be carried out to resolve the issue relating to the stability of the scheme. 


\subsection{Thesis Outline}

The outline for the rest of the thesis is as follows;

Chapter two is a background chapter. It presents the equations of the model and boundary condition that we intend to discretize in the subsequent chapters. It also presents a concise description of the diffusive-viscous wave behaviour, including details on a simple point source, quality factor and active attenuation properties, and the concept of seismic impulse response. This chapter as well includes a discussion of computational methods that are usually used for seismic wave modelling, examining (concisely) geometric methods, and dealing with some other wave-based methods that have been used in the simulation of seismic waves. A detailed review of related work in the area of finite volume methods, not limited to, seismic waves is also presented in this chapter.

Chapter three is an introduction to the application of finite volume methods for diffusiveviscous wave modelling. We provide a detailed discussion of the discretization process, and consider the implications of its accuracy on the quality of the final computed solution. It also presents methods for the control volume integration, which includes the midpoint rule (one-point Gaussian quadrature) and the Gaussian quadrature. A review of the function reconstruction methods and the shape function method for interpolation are also presented in this chapter. This concludes with a detailed review of high-order time integration method applied to the finite volume scheme and focuses on the Runge-Kutta total variation diminishing (TVD) time integration method.

Chapter four considers the implementation phase of the proposed finite volume schemes in two dimensions. It initially provides an introduction to two dimensional structured meshes, and subsequently reviews the process of designing control volumes and subcontrol volumes in Cartesian meshes. Some conventions that guarantee the appropriate and efficient processing of every element are also reviewed. This chapter as well discusses integration and interpolation for two-dimensional problems. The final finite volume discretization in two dimensions are finally provided in this chapter.

Chapter five presents results of the numerical experiment in two-dimensions. Its reviews 
the modelling of diffusive-viscous waves in two dimensions with a simple Cartesian mesh. Flux approximations using the function reconstruction methods and time integration using the Runge-Kutta TVD time integration method for the scheme are discussed in details, including source and receiver implementation. This chapter further reviews the accuracy of the numerical scheme presented.

Chapter six considers the implementation phase of the proposed finite volume schemes in three dimensions. It initially provides an introduction to three dimensional structured meshes, and subsequently reviews the process of designing control volumes and subcontrol volumes in Cartesian meshes. Some conventions that guarantee the appropriate and efficient processing of every element are also reviewed. This chapter as well discusses integration and interpolation for three-dimensional problems. The final finite volume discretization in three dimensions are finally provided in this chapter.

Chapter seven presents results of the numerical experiment in three-dimensions. Its reviews the modelling of diffusive-viscous waves in three dimensions with a simple Cartesian mesh. Flux approximations using the function reconstruction methods and time integration using the Runge-Kutta TVD time integration method for the scheme are discussed in details, including source and receiver implementation. This chapter further presents stability investigations of the numerical scheme.

In Chapter eight, we provide concluding remarks and some recommendations and insights into future research in this area. 


\section{Chapter 2}

\section{Background}

In this chapter, we review the mathematical equations describing the diffusive-viscous wave model, including the primary partial differential equations of interest, that is, the 1D, 2-D and 3-D diffusive-viscous wave equations, as well as, models that are appropriate for the diffusive-viscous wave equation, and models for boundary conditions. We also discuss plane waves, reflection coefficients and seismic sources. Finally, we review some of the computational methods (geometric and wave-based) that are commonly used for seismic simulation and modelling.

\subsection{The diffusive-viscous theory}

In this section, we present some equations related to the diffusive-viscous wave models. We begin with the diffusive-viscous wave equations and their derivation. We then discuss propagating wavenumber and attenuation coefficient of the diffusive-viscous waves. The Quality Factor, $Q$ and Active Attenuation Properties (AAP), $\zeta$ and $\eta$ that are relevant to diffusive-viscous waves are then discussed, followed by a brief description of seismic sources relevant to seismic numerical modelling. The subject of boundary conditions is covered next, with models of absorbing type. The aforementioned begins with the classical type boundary conditions and ends with a special kind of absorbing type boundary condition. Finally, we discuss the concept of impulse responses in the context of seismic wave PDE models. 


\subsubsection{The diffusive-viscous wave equation}

The diffusive-viscous theory was initially proposed by Goloshubin and Korneev (2000); V. A. Korneev et al. (2004). The aim of introducing the theory is to help explain the relationship that coexists between the frequency dependence of the reflections and the fluid saturation in a reservoir. The diffusive-viscous wave equation, which considers the diffusivity and viscosity of porous fluid-saturated layers is mathematically illustrated as (Goloshubin \& Korneev, 2000; V. A. Korneev et al., 2004; Mensah et al., 2019)

$$
\frac{\partial^{2} u(x, t)}{\partial t^{2}}+\zeta \frac{\partial u(x, t)}{\partial t}-\eta \frac{\partial^{3} u(x, t)}{\partial x^{2} \partial t}-\nu^{2} \frac{\partial^{2} u(x, t)}{\partial x^{2}}=0
$$

where $u$ is the wavefield; $\zeta$ and $\eta$ are diffusive and viscous attenuation parameters, respectively, which are functions of porosity and permeability of reservoir rocks, the viscosity, and density of the fluid; $\nu$ is the wave propagation velocity in a non-dispersive medium; $t$ is the time; and $x$ is the space variables. The second term in equation (2.1) characterizes a diffusional dissipative force, whereas the third term describes the viscosity. It should be noted that equation (2.1) is an empirical equation that aims to couple low-frequency diffusion with high-frequency wave phenomenon.

He et al. (2008); Mensah et al. (2019) provide an extension of equation (2.1) to 2-D as

$$
\begin{aligned}
\frac{\partial^{2} u(x, y, t)}{\partial t^{2}} & +\zeta \frac{\partial u(x, y, t)}{\partial t} \\
& -\eta\left[\frac{\partial^{3} u(x, y, t)}{\partial x^{2} \partial t}+\frac{\partial^{3} u(x, y, t)}{\partial y^{2} \partial t}\right] \\
& -\nu^{2}\left[\frac{\partial^{2} u(x, y, t)}{\partial x^{2}}+\frac{\partial^{2} u(x, y, t)}{\partial y^{2}}\right]=0 .
\end{aligned}
$$

In order to extend equation (2.2) to 3-D, we include a third spatial variable, $z$ to equation 
(2.2). It can then be written for the 3-D case as

$$
\begin{aligned}
& \frac{\partial^{2} u(x, y, z, t)}{\partial t^{2}}+\zeta \frac{\partial u(x, y, z, t)}{\partial t} \\
& -\eta\left[\frac{\partial^{3} u(x, y, z, t)}{\partial x^{2} \partial t}+\frac{\partial^{3} u(x, y, z, t)}{\partial y^{2} \partial t}+\frac{\partial^{3} u(x, y, z, t)}{\partial z^{2} \partial t}\right] \\
& -\nu^{2}\left[\frac{\partial^{2} u(x, y, z, t)}{\partial x^{2}}+\frac{\partial^{2} u(x, y, z, t)}{\partial y^{2}}+\frac{\partial^{2} u(x, y, z, t)}{\partial z^{2}}\right]=0 .
\end{aligned}
$$

In equation (2.1), equation (2.2), and equation (2.3), the first terms represent inertia. The second and the third terms characterize a diffusional dissipative force and viscous damping, respectively. The fourth and final term describes the elastic portion of the wave propagation.

It is evident that equations (2.1), (2.2), and (2.3), possess the properties of low-frequency seismic effects and time-delay. All three equations can be employed in characterizing the attenuation of compressional waves in a fluid-filled media. This is because they contain the diffusive and viscous terms. It is also essential to state that equations (2.1), (2.2) and (2.3) couple the low-frequency diffusion with the high-frequency wave phenomenon.

Actually, equations (2.1), (2.2) and (2.3) can be derived from the continuum equations and the basic laws of physics. The discussion and detailed derivation of equations (2.1), (2.2) and 2.3) follows next.

\subsubsection{The derivation and discussion of the diffusive-viscous wave equa- tions}

Spanos (2001) derived, based on the continuum theory, the equations for porosity, fluid pressure, and the equations of motion for the fluid and the solid when he proposed a general equation for dilational waves in fluid-saturated porous media. He obtained the resulting equations by taking the divergence of the equations of motion and substituting 
the equations for pressure and porosity into the resulting equations to give

$$
\begin{array}{r}
\frac{\rho_{\eta f}}{\varphi_{0}}\left(\frac{\partial^{2} \varphi}{\partial t^{2}}+2 a_{\eta f} \frac{\partial \varphi}{\partial t}-2 b_{\eta f} \nabla^{2} \frac{\partial \varphi}{\partial t}\right)= \\
-\frac{\rho_{\xi_{f}}}{\kappa_{f}}\left(\frac{\partial^{2} \xi_{f}}{\partial t^{2}}+2 a_{\xi_{f}} \frac{\partial \xi_{f}}{\partial t}-2 b_{\xi_{f}} \nabla^{2} \frac{\partial \xi_{f}}{\partial t}-\nu_{\xi_{f}}^{2} \nabla^{2} \xi_{f}\right)
\end{array}
$$

where $\xi_{f}$ represents the fluid pressure, $\varphi$ is the porosity and $\kappa_{f}$ represents the bulk modulus of the fluid. Refer to Spanos (2001, pages 194-195), for an understanding of the physical meaning of the variables applied in equation (2.4) above.

If an assumption is made that the porosity is constant even with the evolution in time, equation (2.4) then becomes

$$
\frac{\partial^{2} \xi_{f}}{\partial t^{2}}+2 a_{\xi_{f}} \frac{\partial \xi_{f}}{\partial t}-2 b_{\xi_{f}} \nabla^{2} \frac{\partial \xi_{f}}{\partial t}-\nu_{\xi_{f}}^{2} \nabla^{2} \xi_{f}=0
$$

where $a_{\xi_{f}}$ has a dependence on the shear modulus of the fluid, the porosity and permeability of the solid, the fluid density, and the solid-fluid diffusion ratios, which corresponds to $\zeta$ in equations (2.1), (2.2) and (2.3). $b_{\xi_{f}}$ also represents the bulk modulus of the solid, the shear modulus of the fluid, the fluid viscosity, the porosity of the solid, and the solid-fluid diffusion ratios, which corresponds to $\eta$ in equations (2.1), (2.2) and (2.3). $\nu_{\xi_{f}}^{2}$ represents the fluid density, the porosity, the bulk modulus of the fluid, and the solid-fluid diffusion ratios, which is equivalent to $\nu^{2}$ in equations (2.1), (2.2) and (2.3) when there is zero coupling mass parameter between the fluid and solid.

Based on the mathematical volume averaging theory, and the governing equations for each phase at the pore scale, the equation of megascopic description in a fluid-filled porous media, through fluid flow and elastic deformations of the matrix, is described by the coupled porosity and pressure equation in equation (2.4). In this case, the mathematical averaging theory holds when the porosity is introduced as a dynamic variable in a porous medium. Additionally, the diffusive-viscous wave equations (2.1), (2.2), and (2.3) can be said to be coherent with the 1-D, 2-D and 3-D models for equation (2.5) and the diffusive and viscous attenuation parameters $\zeta$ and $\eta$ can be theoretically computed from equation (2.5). Accordingly, the fluid pressure properties in fluid-filled porous media are 
characterized by the diffusive-viscous wave equation.

\subsubsection{The propagating wavenumber and attenuation coefficient of the diffusive-viscous wave}

A relevant wave-like solution is a plane wave, which is, a wave that is defined over the whole space, and has a steady rate of oscillation that changes, and propagates along a single direction (Carcione, 2007). Equation (2.2) assumes the viscosity along the $x$ and $y$ directions are the same. Equation 2.3 also assumes the viscosity along the $x, y$, and $z$ directions are also the same. These conditions are always true in homogeneous layered media. We derive a harmonic plane wave solution for equation (2.3) in 3-D, which is of the form described below

$$
u=e^{i\left(\tilde{k}_{x} x-\tilde{k}_{y} y-\tilde{k}_{z} z\right)} e^{i \omega t}
$$

where $\omega$ represents the angular frequency and $\tilde{k_{x}}, \tilde{k_{y}}$, and $\tilde{k}_{z}$ representing the wavenumbers in the $x-, y-$, and $z$-directions respectively in units of $1 / m$. It is worth mentioning that $\tilde{k}^{2}=\tilde{k_{x}^{2}}+\tilde{k_{y}^{2}}+\tilde{k_{z}^{2}}$. The complex wavenumber is given by $\tilde{k}$, and it can be written in its real and imaginary parts as

$$
\tilde{k}=k+i \varepsilon
$$

where $k$ describes the propagation wavenumber, $\varepsilon$ represents the diffusive-viscous wave attenuation coefficient and $i=\sqrt{-1}$.

We then substitute equation (2.6) into equation (2.3) to give

$$
(i \omega)^{2}+\zeta(i \omega)-\eta\left[\left(i \tilde{k}_{z}\right)^{2}+\left(i \tilde{k}_{y}\right)^{2}+\left(i \tilde{k}_{x}\right)^{2}\right](i \omega)-\nu\left[\left(i \tilde{k}_{z}\right)^{2}+\left(i \tilde{k}_{y}\right)^{2}+\left(i \tilde{k}_{x}\right)^{2}\right]=0
$$

Let,

$$
\tilde{k}_{x y}=\tilde{k}_{y}+\tilde{k}_{x}
$$


Then, from equation (2.8) and equation (2.9)

$\tilde{k}_{z}^{2}=\frac{\left[\left(\omega^{2}-\nu^{2}\left(\tilde{k}_{x y}^{2}\right)\right) \nu^{2}-\left(\omega \zeta+\left(\tilde{k}_{x y}^{2}\right) \omega \eta\right) \omega \eta\right]-i\left[\left(\omega^{2}-\nu^{2}\left(\tilde{k}_{x y}^{2}\right)\right) \omega \eta+\nu^{2}\left(\omega \zeta+\left(\tilde{k}_{x y}^{2}\right) \omega \eta\right)\right]}{\nu^{4}+\eta^{2} \omega^{2}}$

Taking the form of complex value, $\tilde{k}_{z}$ can be written as

$$
\tilde{k}_{z}=k+i \varepsilon
$$

where $k$ and $\varepsilon$ are real values, $\varepsilon$ is the attenuation coefficient, and $k=\omega / \nu$.

It is imperative to represent $\tilde{k}^{2}$ as

$$
\tilde{k}^{2}=\frac{\omega^{2}-i \zeta \omega}{\nu^{2}+i \eta \omega}=\frac{\left(\nu^{2} \omega^{2}-\zeta \eta \omega^{2}\right)-i\left(\eta \omega^{3}+\zeta \omega \nu^{2}\right)}{\nu^{4}+\eta^{2} \omega^{2}}=\tilde{K}_{\Re}+i \tilde{K}_{\Im}
$$

where, $\tilde{K}_{\Re}=\frac{\nu^{2} \omega^{2}-\zeta \eta \omega^{2}}{\nu^{4}+\eta^{2} \omega^{2}}, \tilde{K}_{\Im}=-\frac{\eta \omega^{3}+\zeta \omega \nu^{2}}{\nu^{4}+\eta^{2} \omega^{2}}$ denote the real and imaginary parts of $\tilde{k}^{2}$.

Equation (2.11) also give the following

$$
\tilde{k}_{z}^{2}=k^{2}+i 2 k \varepsilon-\varepsilon^{2}
$$

Dividing through by $k^{2}$, equation (2.13) can be expressed as

$$
\left(\tilde{k}_{z} / k\right)^{2}=1+i 2(\varepsilon / k)-(\varepsilon / k)^{2} .
$$

We know that

$$
Q=\frac{\omega}{2 \varepsilon \nu}=\frac{k}{2 \varepsilon}
$$

Then from equation (2.15),

$$
\frac{\varepsilon}{k}=\frac{1}{2 Q}
$$

In most instances, the value of $Q$ is between 10 and $10^{3}$. Then, $\frac{1}{2 Q}<<1$. In equation 
(2.14), we can ignore the influence of $\left(\frac{\varepsilon}{k}\right)^{2}$. Then,

$$
\tilde{k}_{z}^{2}=k^{2}+i 2 k \varepsilon
$$

From equations (2.10) and (2.17),

$$
\begin{gathered}
k= \pm \sqrt{\frac{\left(\omega^{2}-\nu^{2}\left(\tilde{k}_{x y}^{2}\right)\right) \nu^{2}-\left(\omega \zeta+\left(\tilde{k}_{x y}^{2}\right) \omega \eta\right) \omega \eta}{\nu^{4}+\eta^{2} \omega^{2}}} \\
\varepsilon=\frac{-\left[\left(\omega^{2}-\nu^{2}\left(\tilde{k}_{x y}^{2}\right)\right) \omega \eta+\nu^{2}\left(\omega \zeta+\left(\tilde{k}_{x y}^{2}\right) \omega \eta\right)\right]}{2 k\left[\nu^{4}+\eta^{2} \omega^{2}\right]} \\
=\frac{-\omega^{3} \eta-\nu^{2} \omega \zeta}{2 k\left[\nu^{4}+\eta^{2} \omega^{2}\right]} \\
=\frac{\omega^{3} \eta-\nu^{2} \omega \zeta}{2\left[\nu^{4}+\eta^{2} \omega^{2}\right]} \cdot\left(\frac{\left(\omega^{2}-\nu^{2}\left(\tilde{k}_{x y}^{2}\right)\right) \nu^{2}-\left(\omega \zeta+\left(\tilde{k}_{x y}^{2}\right) \omega \eta\right) \omega \eta}{\nu^{4}+\eta^{2} \omega^{2}}\right)^{-\frac{1}{2}}
\end{gathered}
$$

In the case of downward continuation, $k$ is negative in equation (2.18)

The attenuation parameters of the diffusive-viscous waves determine the magnitude of the attenuation coefficient, $\varepsilon$ in equation (2.15), that is, either positive or negative. Not only do both the propagation wavenumber, $k$, and the attenuation coefficient, $\varepsilon$, depend on the parameters of the medium, but they also rely significantly on frequency.

We take a step further to obtain the complex velocity from equation (2.8), and it is given as

$$
V \equiv \frac{\omega}{\tilde{k}}=\frac{\omega}{\Re(\tilde{k})}=\sqrt{\frac{\nu^{2}+i \eta \omega}{1-[i(\zeta / \omega)]}}
$$

In Carcione (2007), the phase velocity, $V_{\text {phase }}$ and quality factor, $Q$ were both studied extensively, and were described as

$$
\begin{array}{r}
V_{\text {phase }}=\left[\Re\left(\frac{1}{V}\right)\right]^{-1}, \\
Q=\frac{\Re\left(V^{2}\right)}{\Im\left(V^{2}\right)}=\frac{\left(\nu^{2}-\eta \zeta\right) \omega}{\eta \omega^{2}+\zeta \nu^{2}}
\end{array}
$$


where $\Re$ and $\Im$ respectively denote the real and imaginary parts of a complex number. Equations (2.20) and (2.21) show that not only does the phase velocity, $V_{\text {phase }}$ and the quality factor, $Q$ depend on the diffusive and viscous attenuation properties, $\zeta$ and $\eta$, but they as well rely on frequency. We can then obtain the acoustic wave equation from the phase velocity and wavenumber. To achieve this, we simply set both diffusive and viscous attenuation parameters, $\zeta$ and $\eta$, to zero. In doing so, the quality factor, $Q$, approaches infinity, describing a non-attenuative medium.

\subsubsection{The Quality Factor, $Q$ and Active Attenuation Properties (AAP), $\zeta$ and $\eta$}

Understanding the characteristics of equation (2.3) in a 3-D scenario is essential; hence, a comparison of numerical results obtained from diffusive-viscous waves to those obtained from visco-acoustic waves in the subsequent section. Thus, it is crucial to establish a connection between the active attenuation properties in equation (2.3) and the Quality Factor, $Q$ in a visco-acoustic medium since the attenuation parameters of diffusive-viscous waves depend primarily on the AAP, $\zeta$ and $\eta$.

We obtain a corresponding pseudo quality factor, $Q_{d v w}$ to the diffusive-viscous wave equation (2.3) by the complex velocity as described in Carcione (2007)

$$
Q_{d v w}(f ; \zeta, \eta)=\frac{\Re\left(V^{2}\right)}{\Im\left(V^{2}\right)}
$$

where, $f$ is the frequency, $\Re$ and $\Im$ represent the real and imaginary parts of a complex number, respectively.

One way to obtain the complex velocity of equation (2.3) is by making use of plane harmonic wave $\exp [i(\tilde{\mathbf{k}} \cdot x-\omega t)]$ with angular frequency, $\omega$, wave number, $\tilde{\mathbf{k}}$, and spatial coordinate, $x$ (refer to Zhao, Gao, \& Zhao, 2014). An observation from equations (2.20) and (2.22) is that the complex velocity is frequency-dependent; hence, $Q_{d v w}$ also depends on frequency. Assuming the density and velocity of the medium are known, the unknown model effective properties, that is, the diffusive and viscous attenuation properties, $\zeta$ and 
$\eta$ can be estimated using an inversion method. The calculated and observed values, $Q_{\text {calc }}$ and $Q_{o b s}$, respectively, denote $Q_{d v w}$ and $Q_{v i s}$ accordingly as

$$
\begin{gathered}
Q_{c a l c}=Q_{d v w}(f ; \mathbf{h}) \\
Q_{o b s}=Q_{v i s}(f)
\end{gathered}
$$

where $\mathbf{h}=\left[\begin{array}{ll}\zeta & \eta\end{array}\right]^{\top} ; Q_{v i s}$ represents the Quality Factor of the visco-acoustic medium (see Futterman, 1962; Ganley, 1981) and is defined as

$$
Q_{v i s}=Q_{0}\left[1-\frac{1}{\pi Q_{0}} \ln \epsilon \frac{f}{f_{0}}\right]
$$

where $Q_{0}$ is the quality value, $f_{0}$ represents the reference frequency and $\ln \epsilon=0.5772$. $Q_{c a l c}$ and $Q_{o b s}$, which are also referred to as sampling values, are expressed respectively as

$$
\begin{aligned}
Q_{\text {calc }} & =\left[Q_{\text {calc }}\left(f_{i}\right)\right]^{\top} \\
Q_{\text {obs }} & =\left[Q_{\text {obs }}\left(f_{i}\right)\right]^{\top}
\end{aligned}
$$

where $i=1, \ldots, N$; and $f_{i}$ in equation (2.25) represent the sampling frequencies. The error, $Q_{\text {error }}$ are obtained from observed and calculated values as

$$
Q_{\text {error }}=Q_{o b s}-Q_{c a l c}
$$

The so-called Objective Function, $B$, is then described as

$$
B(\mathbf{h})=\frac{1}{2} Q_{\text {error }}^{*} Q_{\text {error }}
$$

where * represents the conjugated operation.

The AAP, $\zeta$ and $\eta$ in the diffusive-viscous medium can then be inverted using frequently used inversion methods such as the least square method. 


\subsubsection{Source implementation}

There exist numerous ways by which the numerical grid can be excited by the source term. The most basic is the direct inclusion of the source term in the discrete partial differential equation. As an example, after each time step, we may increment by the source term or the right-hand side of the source term may be considered for solving the linear system in the frequency domain. One has to consider the specific effects caused by the discretisation, depending on the numerical approach implemented.

Alterman and Karal Jr (1968) proposed in their pioneering work, the so-called injection technique, whereby a specific square around the source is defined. This technique helps prevent singularities of solutions around the source since the only field computed within the square is the scattering field. The second field, called the incident field, is approximated at the edges of the square. The latter is subtracted when approximations of the propagations are within the square. It is added when the propagations are estimated outside the square.

In this research, the source model implemented will be based on a primary point source. The so-called Ricker wavelet is applied for this study. A Ricker source is simply a zerophase wavelet, that is represented by the second derivative of the Gaussian function. It can also be represented by the third derivative of the normal probability density function and is often applied in numerical modelling and synthetic seismogram production as a zero-phase embedded wavelet. According to Ricker $(1943,1944)$, it is theoretically a solution of the Stokes differential equation with the Newtonian viscosity effect. It is implemented to the Voigt model, which is the propagation of seismic waves through a viscoelastic homogeneous media. The experimental seismic signals are generally similar to the first derivative of a Gaussian, or even the one-and-a-half derivative of a Gaussian that is not symmetric than to the second derivative, which is symmetric in the temporal domain (Hosken, 1988). Still, in reality, this purely Newtonian viscosity appears to be too simple. However, the so-called Ricker wavelet is still prevalent in the field of seismic investigations, since the various derivatives of a Gaussian function have identical spectra. One can use it at the start of the seismic chain to describe a symmetrical source wavelet 
produced, for example, by the correlation of vibrator sweeps. At the finish of the chain, it very well may be an ideal wavelet displayed on a processed seismic profile, with the goal that the peak of the wave can relate well to a subsurface reflector depth or, in the case of geological interpretation, the time of a target reflection. Therefore, the Ricker source is generally a refined wavelet.

Also in a Gaussian distribution is the amplitude spectrum of a Gaussian function. We can describe the very much same spectra of the first, one-and-a-half and second derivatives mathematically as a Gaussian spectrum which is multiplied by factors that are related to frequency, $\omega, \omega^{3 / 2}$, and $\omega^{2}$, where $\omega$ denotes the angular frequency. Aforementioned frequency-dependent multiplications change the amplitude spectra of the Gaussian function from symmetric to asymmetric. Nevertheless, these asymmetric spectra can physically suggest the frequency-dependent attenuation characteristic of seismic waves propagated through a visco-elastic media.

In the time domain, the Ricker wavelet is characterised by a single property which is the dominant frequency. This dominant frequency is the peak frequency in the amplitude spectrum. The peak frequency, which is also the geometric centre of the frequency band happens not to be the central frequency since the amplitude spectrum of the Ricker wavelet is asymmetric. The central frequency and the frequency band of the Ricker wavelet are still not analytically resolved.

Ricker (1953) defined the Ricker wavelet in time domain as

$$
s(t)=\left(1-\frac{1}{2} \omega_{d}^{2} t^{2}\right) \exp \left(-\frac{1}{4} \omega_{d}^{2} t^{2}\right)
$$

where $t$ is the time, and $\omega_{d}$ is the dominant angular frequency (in radians per second). The Fourier transform of the Ricker wavelet can be given as

$$
\Re(\omega)=\frac{2 \omega^{2}}{\sqrt{\pi \omega_{d}^{3}}} \exp \left(-\frac{\omega^{2}}{\omega_{d}^{2}}\right)
$$

$|\Re(\omega)|=\Re(\omega)$ is the real frequency spectrum which has a non-negative value. It is therefore merely the component of the Fourier transform of the even part of the Ricker wavelet. 


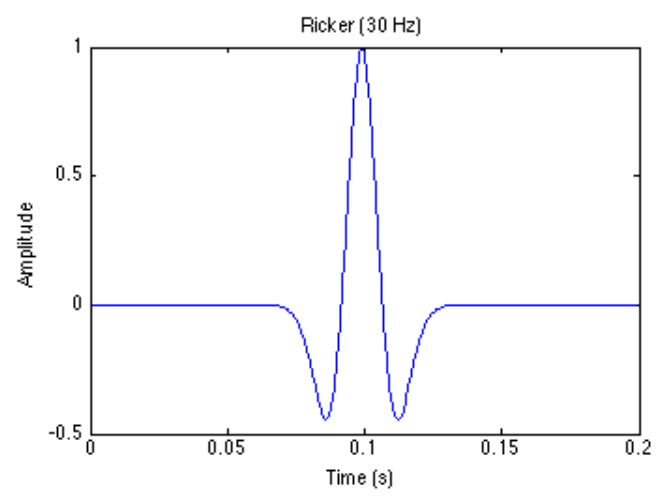

(a) The Ricker wavelet $s(t)$

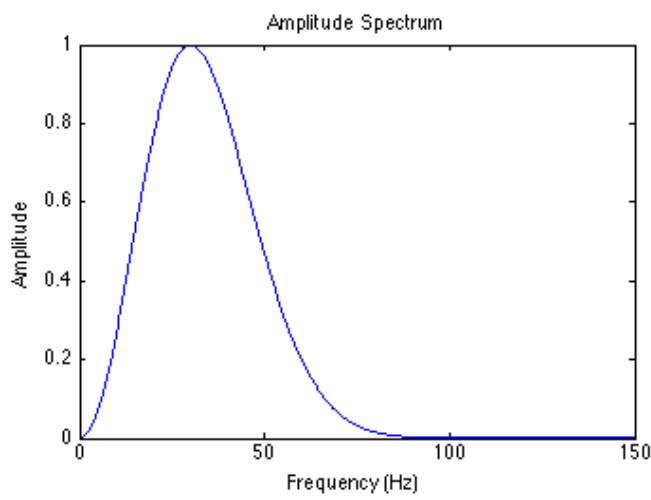

(b) The frequency spectrum, $\Re(\omega)$

Figure 2.1: The Ricker wavelet $s(t)$, and the frequency spectrum, $\Re(\omega)$, with the dominant angular frequency of $60 \pi \mathrm{rad} / \mathrm{s}$

Any delay in the time domain is likely to influence the phase only, and not the magnitude. Thus, we may refer to equation (2.29) as the amplitude spectrum of the Ricker wavelet. To verify that the dominant angular frequency, $\omega_{d}$ is the same as the peak frequency with the highest spectral content, we only set the derivative of the amplitude spectrum to zero, that is, $d \Re / d \omega=0$. Figure (2.1) shows that the Ricker wavelet is symmetrical in the time domain and asymmetrical in the frequency domain with a dominant angular frequency of $60 \pi \mathrm{rad} / \mathrm{s}$ (corresponding to $30 \mathrm{~Hz}$ of regular frequency).

\subsubsection{Boundary conditions}

A boundary condition represents the set of equations applied along a domain boundary to achieve a particular solution to the problem. It is vital to create a distinction between boundary conditions at the domain boundary, physical requirements, and geometric constraints. A physical requirement can be thought off as an "Inlet", a "Wall", or an "Outlet", while geometric constraints can be related to "Symmetry" and "Periodicity". The boundary condition, for any given physical requirement, relies on the equation that is solved and the known variables in the equation solved, with other possible options. A range of boundary conditions will apply to different physical conditions. For example, it is likely to impose a specified pressure and velocity direction at an inlet or to specify the velocity components without allocating any value for the pressure component. One boundary condition, otherwise, illustrates a geometric requirement, often imposed to minimise the 
size of the computational domain. For example, a zero normal flux is the only boundary condition implementable along a line of symmetry.

In most cases, the boundary conditions implemented fall under on the following three fundamental types:

- a Dirichlet boundary condition, where the unknown variable is given at the boundary;

- a Neumann boundary condition, where the flux given in the conservative equation is described at the boundary faces.

- a Robin-type (or mixed) boundary condition, where a constitutive relation expresses the unknown variable and the flux at the boundary.

There exists a fourth type of boundary condition, often referred to as the absorbing boundary condition $(\mathrm{ABC})$. This type of boundary conditions is widely applicable in the field of numerical methods. Most seismic wave propagation problems involve unbounded regions, which are difficult to model computationally. An alternative is to employ the boundary condition mentioned above and to make the simulation region large enough. The simulation must be terminated before reflections from the boundary begin to occur in the area of interest. The disadvantage of this method is an increase in the computational cost of the simulation concerning the region of simulation. Resorting to a boundary condition that absorbs waves and reflects the smallest energy as possible is the best approach. An absorbing boundary condition can be achieved by discretising the first-order wave equation at the end-points of the region and is referred to as the Mur boundary condition (see Clayton \& Engquist, 1977; Zheng, Kishk, Glisson, \& Yakovlev, 2006, for details on Mur boundary condition). We impose the following partial differential equation (PDE), in $1-\mathrm{D}$, at the domain boundaries

$$
\frac{\partial u}{\partial x}=-\frac{1}{c} \frac{\partial u}{\partial t}
$$

The solutions of equation (2.30) is of the form $u(x, t)=u_{0}(x-c t)$, which represents a wave of arbitrary shape propagating to the right as time evolves and this causes waves to 
propagate out of the simulation region without reflection. One has to be mindful when discretising the equation (2.30) since the approximations for the temporal and spatial derivatives in the first-order wave equation must be evaluated at the same point. To achieve this, we must resort to averaging of sample values of $u$, that is,

$$
\frac{1}{2}\left(\frac{u_{N}^{k}-u_{N-1}^{k}}{\Delta x}+\frac{u_{N}^{k+1}-u_{N-1}^{k+1}}{\Delta x}\right)=-\frac{1}{2 c}\left(\frac{u_{N}^{k+1}-u_{N}^{k}}{\Delta t}+\frac{u_{N-1}^{k+1}-u_{N-1}^{k}}{\Delta t}\right)
$$

where $N$ is the number of nodes, $t$ represents time, $x$ represents the $x-$ direction and $k$ is the number of time steps. By resorting to the averaging of the derivatives at two different positions, both approximations of the derivatives in equation 2.31 are evaluated at the location $\left(x_{N}-\Delta x / 2, t_{k}+\Delta t / 2\right)$. Solving for $u_{N}^{k+1}$ results to

$$
u_{N}^{k+1}=u_{N-1}^{k}+\frac{\beta-1}{\beta+1}\left(u_{N-1}^{k+1}-u_{N}^{k}\right)
$$

where $\beta=c \Delta t / \Delta x$ and $c$ is the velocity in $m / s^{2}$. Similarly, the boundary condition at $x=0$ can be determined. The Mur ABC is a perfect absorber for 1-D problems. Readers are adviced to refer to Zheng et al. (2006) for a detailed study on the implementation of first and second-order Mur ABC for 2-D problems.

\subsubsection{Seismic impulse responses}

The key topic in the investigation and reproduction of seismic studies is the concept of a seismic impulse response (SIR). A seismic impulse response suggests a signal that would be recorded at some offset position (using seismic receivers such as geophones) in reaction to an impulsive point-source situated at some other source position. This serves as a partial signature of the seismic source and can be applied straightforwardly to generate artificial vibrations through the convolution operation in time.

To illustrate this concept, we consider equation (2.1) on a domain, $\Omega$ with some related boundary conditions over the boundary surface, and with the initial conditions on the displacement field such that $u=u_{t}=0$ for $t=0$.

We consider the input to the linear system to be $x_{i n}(t)=\Upsilon_{s}(t)$ at the source location, $x_{0}$, 
and $x_{\text {out }}(t)=\Phi\left(x_{1}, t\right)$ as the output of the linear system, where $x_{1}$ denotes the receiver position. With the diffusive, viscous, and velocity parameters constants, it is possible to consider the input/output system as a signal processing block diagram as given in figure (2.2). In this regard, the block that represents the domain can be regarded as a filter, and the transformation from $x_{i n}(t)$ to $x_{\text {out }}(t)$ is referred to as a filtering operation. Because

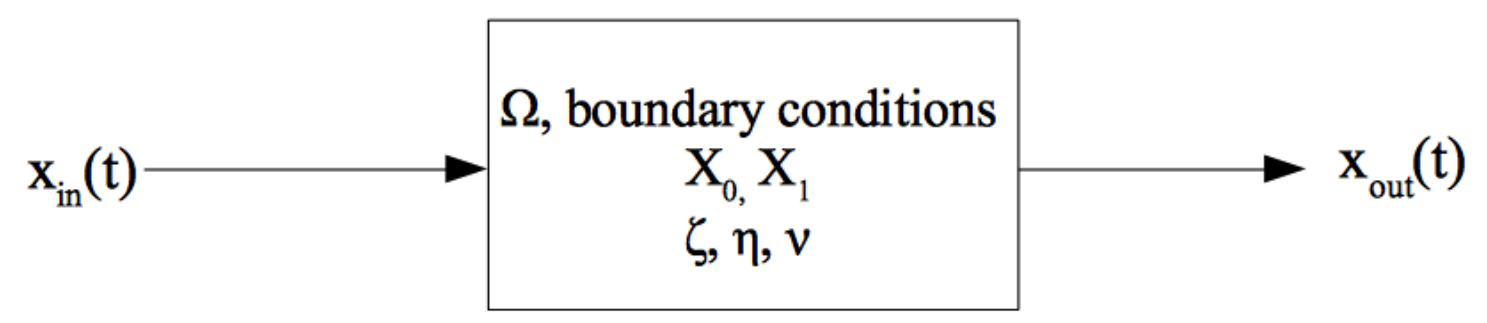

Figure 2.2: Description of a domain as a time-domain filter

equation (2.1) is both linear and time-dependent, this filter can be completely represented by its impulse response. To obtain the impulse response of the system, a Dirac delta is used as an input and $\hbar(t)$, which denotes the output is the impulse response of the filter. The acquisition of the impulse response is illustrated in figure (2.3). The convolution



Figure 2.3: Description of the method to acquire the impulse response

operation in equation (2.33) can be employed for the calculation of the output $x_{\text {out }}(t)$ to the filtering operation given in figure (2.2), provided this impulse response can be obtained.

$$
x_{\text {out }}(t)=\left(x_{\text {in }} * \hbar\right)(t)=\int_{-\infty}^{\infty} x_{i n}(\tau) \hbar(\tau-t) d \tau,
$$

where $*$ is the convolution operator. It is fairly easy to express the convolution operation in equation (2.33) in the frequency domain using multiplication as

$$
\hat{X}_{\text {out }}(\omega)=\hat{X}_{\text {in }}(\omega) \hat{\lambda}(\omega)
$$


where

$$
\hat{X}_{i n}(\omega)=\int_{-\infty}^{\infty} x_{i n}(t) e^{-j \omega t} d t
$$

and essentially, $\hat{\lambda}(\omega)$ represents a frequency-domain signature of the domain in question. Ultimately, it is worth noting that the limits of integration in the convolution operations in equations (2.33) and (2.35) can be decreased to $[0, t]$ if only $x_{i n}(t)=0$ for $t=0$.

\subsection{Computational methods for diffusive-viscous waves in the time domain}

In this section, we present an overview of some computational methods that can be employed in the simulation of diffusive-viscous waves. We begin with the standard geometric methods, then numerical methods for solving the diffusive-viscous wave equation and related partial differential equations (PDEs), like the finite-difference, finite-element, finite-volume, and boundary element methods follow next.

\subsubsection{Geometric methods}

These methods are based on geometrical acoustics (GA), which is a term for methods extensively applied in room acoustics, underwater acoustics and outdoor sound propagation. GA are based on the premise that the amplitude of a wavefront differs little over a distance, along the wavefront, that is akin to the wavelength, and the radii of curvature of the wavefront are considerably more significant than the wavelength (Pierce \& Beyer, 1990). Most substantially, this theory can not model the diffraction phenomenon, for example, at a sharp edge where there is an abrupt transition in the direction of a diffracted wave. GA perceives a sound wave, in a homogeneous media, as propagating with a constant speed along straight lines or rays discharged from a source. With speed of sound gradients in a non-homogeneous media, such rays become curved and by that, model refraction through a medium.

Based on this geometrical scheme, there has been the development of various simulation techniques over the years. Still, this research only deals with the two main methods: the 
ray-tracing method (Borish, 1984; Krokstad, Strom, \& Sørsdal, 1968) and the imagesourcing approach (Allen \& Berkley, 1979; Borish, 1984). Readers are advised to refer to Savioja and Svensson (2015) for an exhaustive review of the other different types of geometric methods available presently.

The primary objective of both ray-tracing and image-source methods is to reconstruct some or all possible paths taken by a ray originating from a point source location and arriving at some receiver position, which is treated as a small volume. These methods usually vary in the approach by which the path reconstruction is carried out, taking into account wall reflections. Once the right path between source and receiver is reconstructed by either method, the distance travelled by ray, combined with the velocity, and the undertaken reflections defined by absorption and probably scattering coefficients, determine the measure of energy that enters from that ray during some period.

It is possible to incorporate frequency-dependent characteristics in the following methods by assuming that the energy that is related with each ray applies to some frequency band of a given width. This kind of frequency-dependency demands numerous simulations for each frequency band of interest. A bank of digital filters can later be used to obtain the individual recombined responses. Another approach to include frequency-dependence into geometric methods is to define the ray as a migrating Dirac delta impulse and to describe a wall reflection as a convolution of the Dirac delta with an impulse response of the frequency response of the wall. New convolutions can evaluate reflections from numerous wall materials with detailed wall impulse responses (Aretz, 2012).

\subsubsection{Ray-tracing method}

In the simple ray-tracing process (Sheaffer, Fazenda, et al., 2014; Krokstad et al., 1968), the usual way of tracing ray-paths from the source to the receiver is by emitting numerous rays in different directions and verifying if they arrive at the receiver with time. Since the surety of the propagation direction of the right path cannot be guaranteed, it is logical to spread the propagation directions consistently over a sphere or to randomise the tracks by some probability distribution (Vorländer, 2007). Because the probability of recognising 
the right path between the source and pointwise receiver position will usually be quite limited, it is logical for the receiver to take up some volume (instead of representing a point in space), to expand the possibilities of "gathering" a ray. Rays can continue being traced; they do not automatically discontinue upon arriving at the receiver. The quantity of energy gathered by the receiver can as well rely on the distance of propagation through the receiver volume (Vorländer, 2007).

The ray-tracing method supports non-specular reflections, either by randomly diverting ray reflections or by dividing them into specular and diffuse rays. Once rays decay adequately in energy, they finally "abate" and stop being traced. One can model the direction of a source by weighting the distribution of discharged rays appropriately. Ray-tracing cost, consisting primarily of ray triangle intersection tests, measures with the number of polygons or surface triangles. Surface meshes, per se, are usually abridged before undertaking ray-tracing, depending on previous theories about the essential geometrical specifics of a model that will be intuitively important to acoustic responses (Vorländer, 2013).

Ray-tracing has a disadvantage, which is that not all initial reflections are necessarily traced, and such initial observations are intuitively relevant (Kuttruff, 2016). Thus, the initial reflections are usually estimated with the image-source approach, which is detailed next.

\subsubsection{Image-source approach}

The image-source approach and the ray-tracing method have a similar objective, that is, to gather and temporarily store energy originating from a source. The fundamental distinction is in the tracing of the path for the source-receiver. In the Image-source method, a deterministic approach is a way to calculate the source-receiver paths. Every path is located in the limit of the algorithm that is being processed for an infinite time. A mirror image of the domain is created by reflecting the domain, which is assumed to be polyhedral, through one boundary face. This results in a virtual domain having its virtual source that traces a path to the receiver going through the reflecting wall. The energy of the ray 
arriving at a given time is driven by the distance from the virtual source to the receiver, and the intersection of the path with the wall. The set of the first-order reflections are computed the moment the process is undertaken for all boundary faces.

We refer to each of those virtual domains as the first-order virtual domain. To compute the second-order reflections, we cause the first-order virtual domains to reflect through their boundary faces, resulting in second-order (new) virtual domains. Second-order reflections from second-order virtual sources are then traced to the receivers, considering all recurrent orders of observations. The process is recursive until otherwise terminated by the user. However, it is usually executed to low-orders since the exponential growth of the computational complexity grows with the order of reflection. In this case, Beam tracing does a better work (Noisternig, Katz, Siltanen, \& Savioja, 2008; Laine, Siltanen, Lokki, \& Savioja, 2009). In the case that adjacent walls do not have acute angles, the need for verification of the validity of the path is required (Borish, 1984). The image-source method is not designed to deal with non-specular reflections.

It is worth noting that an image-source approach is a distinct form of the reflection method (Saarelma \& Savioja, 2014). This method was demonstrated to solve the wave equation in the particular instance of a 3-D box with rigid walls in the extent of infinite-order reflections (Allen \& Berkley, 1979).

The image-source method is usually considered to produce early reflections precisely, and it is frequently used in conjunction with the ray-tracing technique to obtain the later observations (Savioja \& Svensson, 2015).

\subsubsection{Finite-difference method}

The finite difference method (FDM) is possibly the earliest numerical method for solving partial differential equations (PDEs) (e.g., Boole, 1880; Sheppard, 1899). It has been employed in addressing numerous problems such as linear and non-linear problems as well as time-dependent and time-independent problems. The FDM is suitable for problems with various types of boundary shapes and conditions as well as for homogeneous and inhomogeneous media. Richardson earlier recognized the mathematical fundamentals 
of the technique (Richardson, 1911), and numerous mathematical books published, discussed the FDM (e.g., Forsythe, Wasow, et al., 1960; G. D. Smith, Smith, \& Smith, 1985). With the advancement of computer technology, FDM established initial applications in geophysics (Alterman \& Karal Jr, 1968), electromagnetics (Yee, 1966), sound synthesis techniques (Ruiz, 1970), as well as electric and magnetic field applications (Binns \& Lawrenson, 2013). The implementation of FDM is not tricky since it involves simple computations in the derivation of discretized equations and in coding the corresponding equations. FDM was the most vital numerical method applied in dealing with real problems (Stoll, 1967; Ryff, Biringer, \& Burke, 1970; Sarma, 1970). With the development and advancement of high-speed computers with considerably high storage capacity, numerous numerical techniques have been developed for solving PDEs.

Nonetheless, since the finite difference method is easily implemented, it is still widely used for solving numerical problems (Mitchell \& WAIT, 1977; G. D. Smith et al., 1985; Minkowycz, Sparrow, Schneider, \& Pletcher, 1988; Schweig \& Bridges, 1984). Comparable to other numerical methods, finite differences intends to succeed in a continuous field method with infinite degrees of freedom by a discretized field with limited regular nodes. The difference quotients estimate the partial derivatives of the unknown functions at a set of finite discrete points. This transforms the original PDE into a set of algebraic equations. The approximate solution of the initial boundary value problem represents the solution of these simultaneous equations.

\subsubsection{Computational costs for the wave equation with finite difference methods}

Before dealing with other numerical methods, it is essential to briefly throw some light on the computational cost of finite difference methods for the wave equation. The computational costs of finite difference schemes for seismic propagation problems, in particular acoustics, can seem heavy, however, it is essential to put these costs into context with the underlying PDE model, in this case, the acoustic model.

We deal with only the second-order form of the PDE since it is often highly computationally expensive than the first-order form assumption of a constant velocity, $c$ (Botts 
\& Savioja, 2013; Webb, 2014). Nearly all of the second-order finite difference methods employed in seismic wave propagation investigations can be expressed as the subsequent matrix recursion

$$
\mathbf{E} \mathbf{u}^{k+1}=\mathbf{F} \mathbf{u}^{k}+\mathbf{G} \mathbf{u}^{k-1}
$$

where $\mathbf{u}^{\mathbf{k}}$ is an $N \times 1$ column vector, describing the whole spatially-discretized acoustic field of any kind at time $t=k$, where $k$ represents the time-step, and $\mathbf{E}, \mathbf{F}, \mathbf{G}$ are $N \times N$ matrices. $N$ is the number of nodes in which the domain is discretized. Usually, E, F, G are represented as sparse matrices, and $\mathbf{E}$ and $\mathbf{G}$ are generally diagonal. Furthermore, $\mathbf{E}$, F, G do not require explicit storage, as in the case of regular grid finite-difference schemes such as the Cartesian scheme, since they are relatively uniform. In such instances, only one row needs to be stored, and a few variations of that row for grid points near the boundary of the domain.

Explicit schemes are commonly used in seismic wave investigations, at which point $\mathbf{E}$ and $\mathbf{G}$ are diagonal matrices. Thus, they can be described as follows

$$
\mathbf{u}^{k+1}=\overline{\mathbf{F}} \mathbf{u}^{k}+\overline{\mathbf{G}} \mathbf{u}^{\mathbf{k}-\mathbf{1}}
$$

where $\overline{\mathbf{F}}=\mathbf{E}^{-\mathbf{1}} \mathbf{F}$ and $\overline{\mathbf{G}}=\mathbf{E}^{-\mathbf{1}} \mathbf{F}$ are rewritten in order to reflect the fact that $\mathbf{E}^{-\mathbf{1}}$ is trivially calculated to represent new sparse matrices, and in addition, $\overline{\mathbf{G}}$ is still diagonal. In such instances, $2 N$ becomes the storage specification for the scheme, because $\mathbf{u}^{k+1}$ can override $\mathbf{u}^{k-1}$ in position. Since the matrices are sparse, and because the primary operation at each time-step is a vector multiplied by a sparse matrix, the complexity of this explicit scheme per time-step is given as $\mathcal{O}(N)$.

Now we consider the specific case of acoustic wave propagation in three spatial dimensions.

$$
\nabla^{2} P=\frac{1}{c} \frac{\partial^{2} P}{\partial t^{2}}
$$

where $\nabla^{2}$ is the Laplace operator, $p$ is the acoustic pressure (the local deviation from the ambient pressure), and $c$ is the speed of sound. By taking into account 3-D spatial sampling as well as the wave equation dispersion relation (associating temporal frequencies 
to spatial frequencies), we discover that $N \propto f^{3}$, where $f$ represents the maximum temporal frequency of interest. Therefore, the required storage is $\mathcal{O}\left(f^{3}\right)$, and $\mathcal{O}\left(f^{3}\right)$ is also the computation per time-step. It is worth noting that for any 3-D domain, the modal density, or the degrees of freedom will be $\mathcal{O}\left(f^{3}\right)$. Notably, the complexity of the continuous case measures with that of the finite difference method, and the computational costs of the finite difference method demonstrate the complexity of the fundamental problem, that is, without taking into account approximation errors. In the event of extended time duration, the computational cost will result in $\mathcal{O}\left(f^{4}\right)$, since the number of time-steps needed for a required time duration will be proportional to $f$, that is, by merely considering temporal sampling (Anné, Tran, \& Symes, 1997).

\subsubsection{Finite-element and finite-volume methods}

Finite Element (Courant et al., 1943; Zienkiewicz, 1977; Ihlenburg, 2006) and finite volume methods (Varga, 1962; Baliga \& Patankar, 1980; Heinrich, 1987; LeVeque, 2002) can both be regarded as an intimately connected extension of the finite difference method (Zienkiewicz \& Oñate, 1992; Peiró \& Sherwin, 2005) (also refer to Thomée, 2001). These schemes can as well be represented in the form of equation (2.36) when used in solving the wave equation, even though the form and structure of their matrices will differ from the finite difference scheme. We discuss these differences in the matrix structures below.

The primary advantage these schemes have over the finite difference approach is the ease in the treatment of unstructured grids, permitting for the adaptation to boundary surfaces which do not align smoothly with axes of the grids. Their demerit over the finite difference method is the complexity they present in practical implementation since they generally need a pre-processing meshing step, hence additional mesh data storage. This extra storage implies that most, if not all, the rows of the matrices $\mathbf{E}, \mathbf{F}, \mathbf{G}$ (see equation 2.36), must be stored. Thus, it as well usually the fact that $\mathbf{E}$ and $\mathbf{G}$ are diagonal. 


\subsubsection{Finite element methods}

In the finite element schemes, the spatial domain is discretized into elements that are polygons or polyhedra in shape, usually tetrahedra or triangles. And so-called local basis functions are selected to connect these elements (Zienkiewicz, 1977). The basis elements are used to estimate the solution, and some assumed chosen residual is reduced, producing a linear system of equation whose weighting coefficients result in an approximation of the unknown variable of interest (e.g., the acoustic field). An essential pre-processing procedure is the matrix construction related to the linear system of equations, as is the preference of the finite element mesh. Two matrices are usually obtained from this process. These are referred to as the mass matrix and the stiffness matrix, which are both sparse as a result of the localized structure of the selected finite element basis functions. Finite element schemes for seismic wave investigations can usually be applied in either the frequency domain (e.g., Shuku \& Ishihara, 1973; Craggs, 1994; Aretz, 2012) or the time domain (e.g., Svensson \& Kristiansen, 2002; Cohen, 2013; Okuzono, Yoshida, Sakagami, \& Otsuru, 2016). In the time domain formulation, $\mathbf{E}$ represents the mass matrix, and the stiffness matrix forms part of $\mathbf{F}$ (see equation 2.36). According to Cohen (2013), the time recursion is implicit when the mass matrix is not diagonal. It can be made explicit with a diagonal mass lumping approach. It is worth noting that a linear system of solution is required when the time recursion is implicit.

\subsubsection{Finite volume methods}

Finite volume methods are based on the weak formulation of the problem, as in with the finite element method. Since the finite volume method is the primary focus of this thesis; it is worthwhile to give a short history of the various types of finite volume methods with their more comprehensive applications in other fields.

\subsubsection{Standard cell-centred method}

The first implementation of the cell-centred finite volume method was proposed about thirty-two years ago, in its contemporary form, to solid mechanics by Demirdžic, Martinovic, and Ivankovic (1988). The cell-centred method obtains its name from the de- 
pendent variable located at the control volume centroids (cell centres); the technique has similarly been referred to as the collocated finite volume method since the dependent variables share their position at the cell centres. In the earlier approach of Demirdžic et al. (1988), he assumed that the displacement would locally differ linearly. A differentiating characteristic of the suggested solution algorithm was the segregation of the surface force term into a compact-stencil implicit term and a broader-stencil explicit term. Subsequently, this resulted in a temporal decoupling of the linear momentum vector equation into three scalar component equations that were separately solved, where the needed coupling was provided by outer fixed-point/Gauss-Seidel/Picard iterations. This kind of solution methodology is referred to as a segregated method since the governing conservation of linear momentum equation is partitioned into three scalar equation in the event of the solution.

The initial 2-D structured grid approach of Demirdžic et al. was later extended to 3-D (Demirdžić \& Muzaferija, 1994), where substantial freedom in accurate mesh description was implemented by assuming general convex polyhedral cells. The cell-centred technique has since been expanded in solving a wide range of solid and multi-physics problems, which include, poroelasticity (Bryant, Hwang, Sharma, et al., 2015; Cardiff et al., 2015; D. Lee et al., 2015; T. Tang, Hededal, \& Cardiff, 2015; Asadollahi, 2017; Manchanda, Bryant, Bhardwaj, Sharma, et al., 2016), anisotropy (Fainberg \& Leister, 1996; Cardiff \& Demirdžić, 2018; Demirdžić, Horman, \& Martinović, 2000; Martinović, Horman, \& Demirdžić, 2001; Martinović, Horman, \& Hajdarević, 2008; Horman, Hajdarević, Martinović, \& Vukas, 2010; Horman, Martinović, Bijelonja, \& Hajdarević, 2012; Cardiff, Karač, \& Ivanković, 2014) and heterogeneous material characteristics (Cardiff, 2012; Tuković, Ivanković, \& Karač, 2013; Carolan, Tuković, Murphy, \& Ivanković, 2013; Bryant et al., 2015; Manchanda et al., 2016), elastoplasticity (Demirdžić \& Martinović, 1993; Dioh, Ivankovic, Leevers, \& Williams, 1994; Maneeratana, 2000; Rente \& Oliveira, 2000; Bašić, Demirdžić, \& Muzaferija, 2005; J. Bressan, Martins, \& Vaz Jr, 2010; Martins, Bressan, Button, \& Ivankovic, 2011; Leonard, Murphy, Karač, \& Ivanković, 2012; Cardiff, Tuković, Jasak, \& Ivanković, 2016; Matos Martins, Button, \& Bressan, 2016; J. D. Bressan, Martins, \& Button, 2017; Cardiff et al., 2017; Martins, Bressan, \& 
Button, 2017) and viscoelasticity (Zarrabi \& Basu, 1999, 2000; Demirdžić, Džaferović, \& Ivanković, 2005; Safari et al., 2016; Das, Mathur, \& Murthy, 2012), fluid-solid interaction (Henry \& Collins, 1993, 1970; Demirdžić \& Muzaferija, 1995; Greenshields, Weller, \& Ivanković, 1999a, 1999b; Greenshields, Venizelos, \& Ivanković, 2000; Ivanković, Karač, Dendrinos, \& Parker, 2001; Schäfer \& Teschauer, 2001; Ivanković, Karač, Dendrinos, \& Parker, 2002; Schäfer, Teschauer, Kadinski, \& Selder, 2002; Cardiff \& Demirdžić, 2018; Torlak, Muzaferija, \& Peric, 2002; Kovacevic, Stosic, \& Smith, 2004; Greenshields \& Weller, 2005; Papadakis \& Giannopapa, 2006; Kovačević, Stošić, \& Smith, 2006; Stosic, Smith, \& Kovacevic, 2005; Shaw, Stone, et al., 2005; Torlak, 2006; Jasak, Jemcov, Tukovic, et al., 2007; Kovacevic, Stosic, Mujic, \& Smith, 2007; Kovacevic, Stosic, \& Smith, 2007; Cardiff \& Demirdžić, 2018; Papadakis, 2008; Kanyanta, Ivankovic, \& Karac, 2009; Jagad, Puranik, \& Date, 2011; Wiedemair, Tuković, Jasak, Poulikakos, \& Kurtcuoglu, 2012; Habchi et al., 2013; I. K. Smith, Stosic, \& Kovacevic, 2014; Tukovic, Cardiff, Karac, Jasak, \& Ivankovic, 2014; Jagad, 2016; Šekutkovski, Kostić, Simonović, Cardiff, \& Jazarević, 2016; Jagad, Puranik, \& Date, 2017, 2018), among others.

Separated from the lineage of Demirdžic et al. (1988) and that of other cell-centred methods, numerous alternatives to the finite volume methods have been developed, and we discuss these next.

\subsubsection{Staggered-grid methods}

The staggered-grid technique was initially proposed by Harlow and Welch (1965) for computational fluid dynamics (CFD). The distinguishing feature of this approach is its grid design: the components of the fundamental solution variable (e.g., $x$ and $y$ components of displacement) are stored at various locations. Additionally, varied sets of control volumes may be employed when the discretized governing equations are integrated into each Cartesian orientation, for example, the $x$-momentum component equation may utilize a distinct grid to the $y$-momentum component equation. The main reason for such staggered-grid methods is the prevention of the "checker-boarding" numerical anomaly, by which there is the appearance of high-frequency discrepancies in the solution variables that are not observed during discretization. It is, however, not trivial to extend these 
staggered-grid methods to general unstructured 3-D meshes. As a result, such approaches experience a decline in popularity.

As presented by Hattel, Hansen and collaborators (Hattet \& Hansen, 1990; J. Hattel, Hansen, \& Hansen, 1993; J. Hattel, Hansen, \& Andersen, 1993; J. Hattel, 1993b, 1993a; J. H. Hattel, 1997; Pryds \& Hattel, 1997; J. H. Hattel, Thorborg, \& Andersen, 1998; J. Hattel \& Pryds, 2001; Thorborg, 2001; J. H. Hattel \& Thorborg, 2003; Thorborg \& Hattel, 2003) where the only underlying variable was displacement, the staggered-grid techniques do not require the implementation of mixed displacement-pressure methods. Hattet and Hansen (1990) originally proposed their staggered-grid technique for thermally induced stress analysis in problems related to casting, and later extended it for dealing with other issues such as thermal stresses in concrete ageing and problems for thermo-elastoplasticity. They further indicated the close relationship of their scheme with the finite difference methods and thus, referred to their technique as "a control volume-based finite difference method". The authors recognized that their approach produced an exquisite formulation for non-constant material characteristics, an advantage of the staggered-grid scheme. Conversely, Tuković et al. (2013) initially extended the approach of Demirdžic et al. (1988) to non-constant material characteristics by a somewhat tedious process. Several substitute staggered-grid schemes have as well been proposed: L. X. Wang and Melnik (2007) presented a staggered finite volume scheme for shape memory alloy analysis operated on 2-D structured rectangular grids. Furthermore, Rajagopal, Srinivasa, and Ponnalagu (2014) studied a layered viscoelastic plate response, applying a single-step explicit staggered-grid finite volume scheme on structured meshes.

\subsubsection{Vertex-centred method}

Fryer, Bailey, Cross, and Lai proposed a vertex-based finite volume scheme for static elastic stress investigations, in addition to the cell-centred and staggered techniques earlier discussed (Fryer et al., 1991). In the vertex-centred methods, the primary unknowns are stored at the mesh vertices, and the governing equation is integrated over the control volumes surrounding each mesh vertex. Fryer et al. initial approach, which was originally referred to as a control volume-unstructured mesh method, could evaluate complex 2-D 
geometry applying both triangular and quadrilateral cells. The approach of Fryer et al. was an improvement of the technique proposed a decade earlier by Baliga and Patankar (1980), who presented a supposed control-volume-based finite element method on unstructured triangular grids for the convection-diffusion equations. They observed the property of local conservation as a characteristic of the control-volume formulation and this property allowed for straightforward physical interpretation.

Numerous authors have investigated in detail, the vertex-based finite volume method and have as well taken a step further to compare this technique with others such as the finite difference, finite element, cell-centred finite volume methods (Filippini, Maliska, \& Vaz Jr, 2014; Vaz Jr, Muñoz-Rojas, \& Filippini, 2009; Hejranfar \& Azampour, 2015; Perre \& Passard, 1995; Selmin, 1993). Voller (2009) provides extensive information on the investigation of control volume methods.

\subsubsection{Godunov-type methods}

Godunov-type finite volume schemes were initially introduced to deal with the solution of hyperbolic differential equations represented by shocks, waves, and solution discontinuities (LeVeque, 2002; Godunov, 1959, 1962; Toro, 2012). Since then, the methods have been extensively applied and is famous for the solution of Euler compressible gas flow equations. The classic technique is applied to systems of conservation laws which are written as systems of first-order hyperbolic PDEs, such as the Euler equations of gas . It determines fluxes at the control volume faces by representing the approximate solution as a Riemann problem at each inter-cell boundary. The resulting discretizations compute the flux at each inter-cell boundary (control volume face) as a weighted average of the flux calculated at each inter-cell boundary.

Trangenstein and Colella initially implemented the Godunov-type schemes to structural problems when the 1-D wave propagation in elastoplastic solids was modelled (Trangenstein \& Colella, 1991; Trangenstein \& Pember, 1992; Trangenstein, 1994). In their technique, the linear momentum vector and the deformation gradient tensor where the original conservation variables considered. 
The approach has later been extended to 3-D unstructured grids in numerous forms, with the discretizations, grid arrangements, and primitive variables being the only distinctions (Miller \& Puckett, 1996; H. Tang \& Sotiropoulos, 1999; Berezovski \& Maugin, 2001; Howell \& Ball, 2002; Berezovski \& Maugin, 2003; LeVeque, 2002; Kluth \& Després, 2008, 2010; C. H. Lee, Gil, \& Bonet, 2013; Maire, Abgrall, Breil, Loubère, \& Rebourcet, 2013; Sambasivan, Shashkov, \& Burton, 2013; Aguirre, Gil, Bonet, \& Carreño, 2014; Aguirre, Gil, Bonet, \& Lee, 2015; Cheng, Toro, Jiang, Yu, \& Tang, 2015; Després \& Labourasse, 2015; Ndanou, Favrie, \& Gavrilyuk, 2015; Sijoy \& Chaturvedi, 2015; Boscheri, Dumbser, \& Loubère, 2016; Loubère, Maire, \& Rebourcet, 2016; Cheng, Jia, Jiang, Toro, \& Yu, 2017; Cheng, Huang, Jiang, \& Tian, 2017; Fridrich, Liska, \& Wendroff, 2017; Georges, Breil, \& Maire, 2017; Castañar, Baiges, \& Codina, 2019; Heuzé, 2017; Haider, Lee, Gil, Huerta, \& Bonet, 2018; Heuzé, 2018; Sevilla, Giacomini, \& Huerta, 2018a, 2018b). A unique attribute of Godunov-type schemes is its ability to adopt to fully explicit solution algorithms, where the standard Courant-Friedrichs-Lewy constraint controls the time increment size (Courant, 1928). Even though the most prevalent type of Godunov-type method is the cell-centred formulations, vertex-centred (Aguirre et al., 2014, 2015) and, currently, face-centred formulations (Sevilla et al., 2018a, 2018b) have as well been investigated. Thus, the scheme is useful for time-dependent hyperbolictype schemes and considerably less efficient for elliptic methods.

Readers are advised to further research on the other methods of finite volumes as it is impossible to acknowledge all approaches. Interested readers can refer to Bašić et al. (2005); Williams, Croft, and Cross (2002); Williams, Slone, Croft, and Cross (2010); Chen, Lou, and Ruan (2007); Jafari, Zebarjad, and Kolahan (2007); Lou, Zhao, Wang, and Wu (2008); Al-Athel and Gadala (2011); R. Wang and Li (2011); R. Wang (2012); De Brauer, Iollo, and Milcent (2016); C. Zhang, Chen, Zhao, Zhang, and Lou (2016); De Brauer, Iollo, and Milcent (2017), just to mention a few. 


\subsubsection{Finite element and finite volume methods for the wave equation: computa- tional costs}

Similar computational complexities as finite difference schemes exist for both finite element and finite volume methods, that is, $\mathcal{O}\left(f^{3}\right)$ and $\mathcal{O}\left(f^{4}\right)$ for storage and operations respectively, for the 3-D scalar wave equation. Nevertheless, the substantive memory costs may surpass those of finite-difference schemes when applying fully unstructured grids, because $\mathbf{E}$ and $\mathbf{F}$ (refer to equation (2.36)) must be stored in full, even though still as a sparse matrix. It is worth emphasizing that this is only valid for approximately the same cell size and ignores accuracy considerations which may permit the implementation of a coarser grid by one approach. When applied as a complement to conventional finite difference methods, on the other hand, extra storage is only required near the domain boundary.

\subsubsection{Boundary element methods}

Boundary element methods (BEM) are elegant means for the analysis of boundary value problems for partial differential equations. They are based on the fact that the solution to an inhomogeneous wave equation boundary-value problem can be expressed in terms of the free-space Green's function, the initial conditions, the boundary conditions and the known source term (if applicable) (Duffy, 2015). This general solution requires a numerical approximation technique since it is represented as an integral equation, which can not be conveniently evaluated. The boundary element methods discretize a general solution to the wave equation, and as such, they do not discretize the wave equation itself. The matrix recursion (equation (2.36)) do not represent these methods.

The integral equation is referred to as the "Kirchhoff integral equation" (KIE). For a time-dependent solution, it is referred to as the "Kirchhoff-Helmholtz integral equation" (KHIE), neglecting source terms and initial conditions. BEM usually applies KIE in the time-domain and KHIE in the frequency-domain. These integral equations indicate that when the wavefield reacts to a point source somewhere within the boundary, as long as the pressure and normal velocity are known ubiquitously on the surface of the boundary, this 
wavefield (pressure or acoustic velocity potential) can be predicted anywhere within the domain. The pressure over the surface of the boundary represents the unknown variable in this problem.

To solve for the unknown variable, any type of element technique can be implemented over the boundary. The boundary surface is discretized into surface elements, and then a set of basis functions are chosen over the elements. The linear system of equation that solves for the wavefield at the boundary, or more precisely, the required weighting coefficients for the reconstruction of the wavefield (pressure) from the basis functions, is obtained when the error is set to zero either at sampling points in the elements or over the whole elements (Galerkin technique). One can get the pressure in the internal domain when the known free-space Green's function is used to transmit the approximation over the boundary back to the central points of interest, once this system is computed over the boundary.

The BEM has the main merit of decreasing the problem by one dimension, signifying a significant reduction in the number of degrees of freedom in the numerical approximation. Nevertheless, the resulting system of equation usually features a full matrix; thus, the solution of the linear system can be computationally expensive, and the storage of this matrix depends much on memory costs. In 3-D problems, the number of degrees of freedom measures as $\mathcal{O}\left(f^{2}\right)$ for BEM, for some maximum frequency of interest, $f$, which is an improvement of the $\mathcal{O}\left(f^{3}\right)$ needed for other grid-based methods. The storage costs are however $\mathcal{O}\left(f^{4}\right)$, due to the typically full matrix required.

\subsection{Summary}

In this chapter, we have presented an overview of PDEs that can be applied in timedomain diffusive-viscous wave modelling, integrating a source term and a boundary condition of absorbing type. Further in this chapter, various techniques to diffusive-viscous wave simulations have also been reviewed. These included geometric methods, and some conventional wave-based methods such as finite difference, finite element, finite volume, and boundary element methods, in addition to the merits and demerits of each technique. 


\section{Chapter 3}

\section{Methodology}

In this chapter, we describe the finite volume method (FVM) adopted in this thesis for solving the diffusive-viscous wave equation (DVWE) described in the previous chapter. We present a second-order spatial discretization scheme which accommodates both structured and unstructured meshes on a Cartesian stencil or with cells of arbitrary shape. Finally, we review time integration schemes applied to the solution of time-dependent diffusive-viscous wave problems.

\subsection{Finite volume method}

There are numerous methods for the numerical solution of the governing equations. A vast majority achieve results with the following three main procedures:

- temporal discretization: this involves the evolution of the solution in time by dividing the whole time interval into a finite number of sub-intervals, referred to as time-steps.

- Spatial discretization: this has to do with the definition of a numerical grid to succeed the regular space with a finite number of discrete elements with computational points at their centroids.

- Equation discretization: this stage replaces the individual components of the governing equations with algebraic expressions which link the variable values at computational points in the grid. 


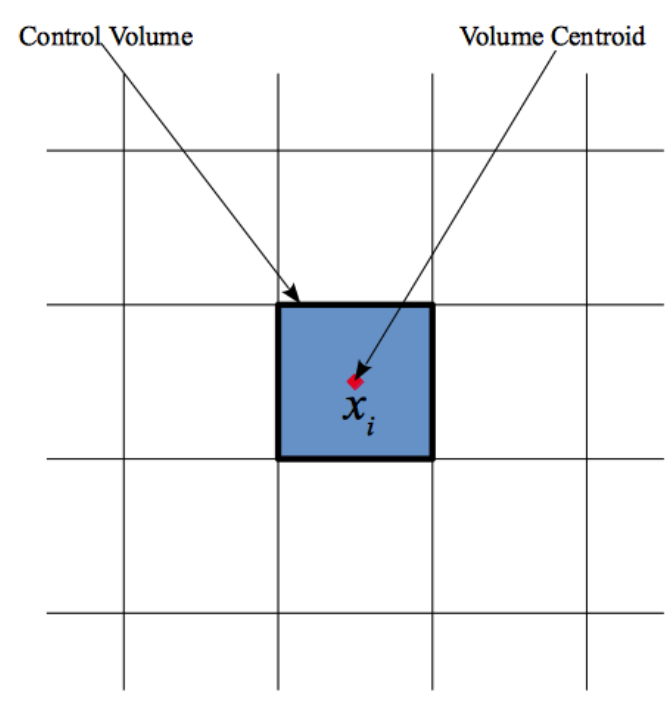

(a)

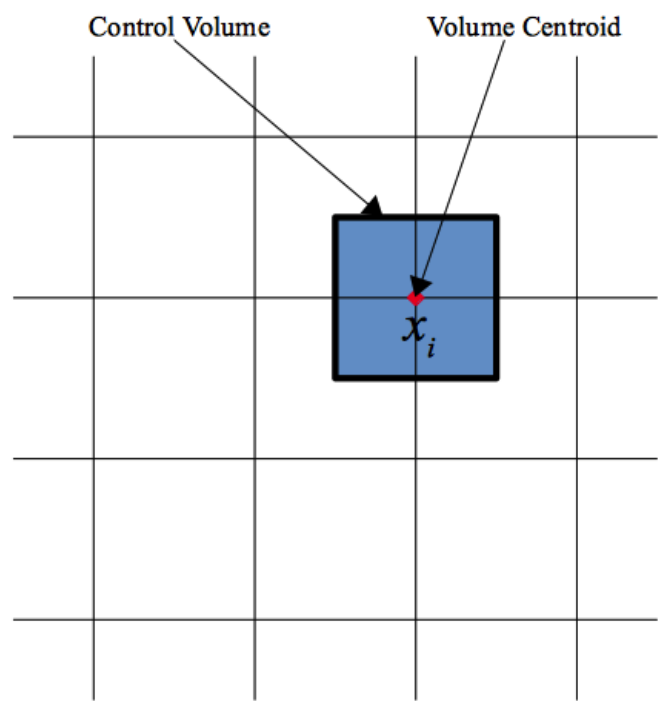

(b)

Figure 3.1: Finite volume method showing (a) cell-centered and (b) vertex-centered control volumes.

We adopt the finite volume method of discretization in the current research. It applies directly the conservation laws, which initially transforms the governing equations into their integral forms as part of the discretization process. We discretize the solution domain by a structured mesh consisting of a finite number of contiguous control volumes (CVs) or cells. The computational points are situated at the centre of each control volume (CV), and each $\mathrm{CV}$ is bounded by several cell faces which forms the control volume surface. A control volume may take any shape as there is no limitation to the structure of a control volume (see figures 3.1, 3.2). A unique data structure based on cell faces is used to achieve this, which provides the connectivity of the data between the cells distributed at the same cell face. It gives the solver the flexibility of dealing with meshes which consist of cells with different topology since the number of cell faces per control volume can differ arbitrarily from one control volume to another. An invariable data structure supports the cell-wise (local) grid refinement as only the list of cell faces require an update. The interface that exists between the refined and non-refined cells can, accordingly, be fully conservatively evaluated. All this presents excellent flexibility concerning the numerical mesh that can be utilized.

A suitable arrangement for the arbitrary structured meshes is employed, which is referred 

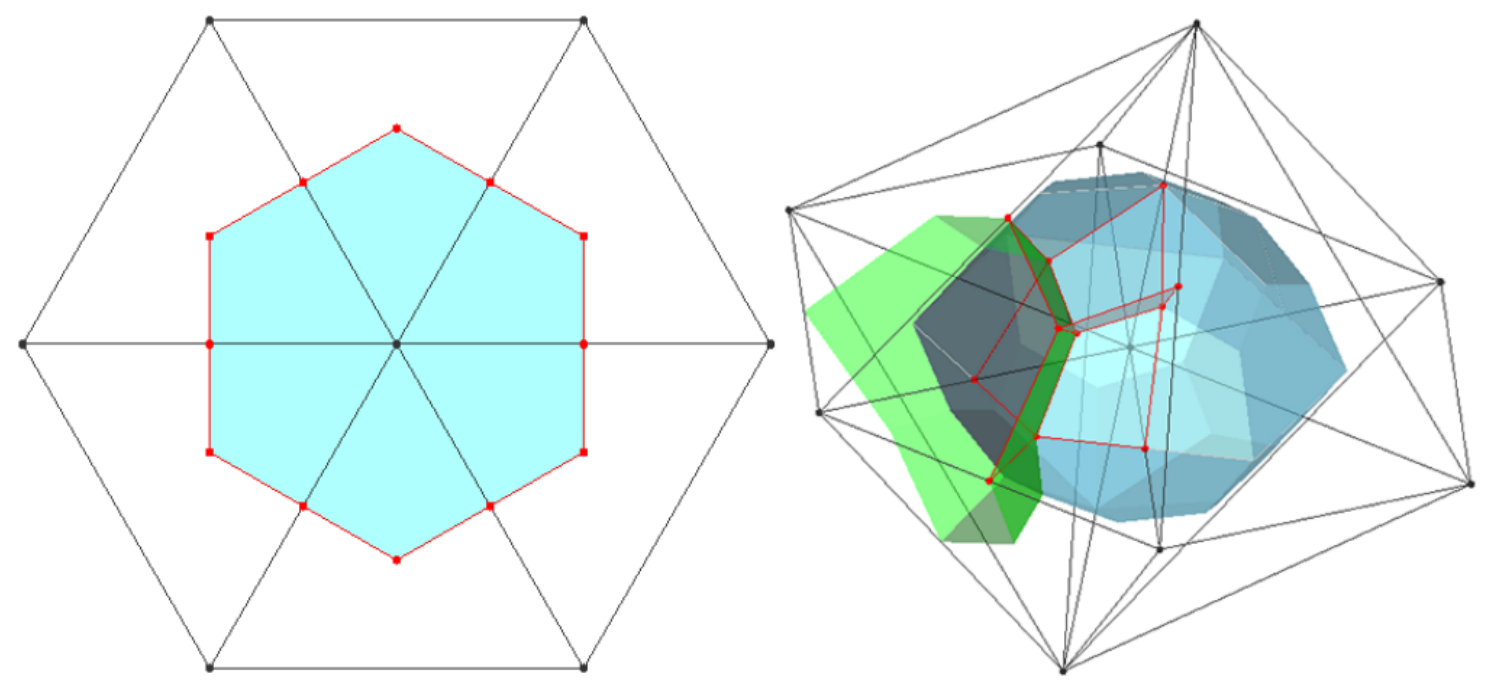

Figure 3.2: Control volume of the vertex-centred finite volume method (a). 2-D triangle cell (b). 3-D tetrahedron cell

to as the non-staggered (collocated) variable distribution and considers all dependent variables as sharing the same control volume. The strong conservation form of the diffusiveviscous wave equations (DVWE) is provided as equations that are solved in a Cartesian coordinate system, which makes the technique insensitive to the non-smoothness nature of the grid (Niceno, 2003). The system of conservation equations obtained from the DVWE is dealt with in a segregated manner, implying that these equations are solved one after the other, and explicitly treating the inter-equation coupling.

For the meantime, assume that there exist $N$ nodes in the mesh and that there exists a control volume $V_{i}$ around each node $\mathbf{x}_{\mathbf{i}}$, with surface $\varsigma_{i}$ and volume $\Delta V_{i}$, such that $V_{i}$ does not overlap and the total mesh volume $V_{\text {total-mesh }}$ is described as

$$
V_{\text {total-mesh }}=\sum_{i=1}^{N} \Delta V_{i} .
$$

Now consider the time-dependent diffusive-viscous wave equation in the velocity-displacement formulation (Mensah et al., 2019)

$$
\begin{gathered}
\frac{\partial u}{\partial t}=v \\
\frac{\partial v}{\partial t}+\zeta \cdot v-\nabla \cdot(\eta \nabla v)-\nabla \cdot\left(\nu^{2} \nabla u\right)=s(t),
\end{gathered}
$$


where $u$ and $v$ are displacement and velocity quantities (wavefields), respectively.

The finite volume method progresses by integrating equations (3.2) and (3.3) over each control volume $V_{i}$ as

$$
\begin{gathered}
\iiint_{V_{i}} \frac{\partial u}{\partial t} d V=\iiint_{V_{i}} v d V \\
\iiint_{V_{i}} \frac{\partial v}{\partial t} d V=-\iiint_{V_{i}} \zeta \cdot v d V+\iiint_{V_{i}} \nabla \cdot(\eta \nabla v) d V \\
+\iiint_{V_{i}} \nabla \cdot\left(\nu^{2} \nabla u\right) d V+\iiint_{V_{i}} s(t) d V
\end{gathered}
$$

The Gauss' Divergence Theorem subsequently allows the second and third right hand side terms in equation (3.5), representing the viscous and velocity fluxes, to be expressed as integrals over the surface $\varsigma_{i}$ given as

$$
\begin{gathered}
\iiint_{V_{i}} \nabla \cdot(\eta \nabla v) d V=\iint_{\varsigma_{i}}(\eta \nabla v) \cdot \hat{\mathbf{n}} d \varsigma, \\
\iiint_{V_{i}} \nabla \cdot\left(\nu^{2} \nabla u\right) d V=\iint_{\varsigma_{i}}\left(\nu^{2} \nabla u\right) \cdot \hat{\mathbf{n}} d \varsigma,
\end{gathered}
$$

where $\hat{\mathbf{n}}$ is the vector normal to the boundary of the control volume. Defining the control volume (CV) averaged values of $u$ and $v$ in equation (3.4), and $v$ and $s$ in equation (3.5) as $\bar{u}_{i}, \bar{v}_{i}$ and $\bar{s}_{i}$, since $u$ and $v$ represent the same quantities, and are given as

$$
\begin{gathered}
\bar{u}_{i}=\frac{1}{\Delta V_{i}} \iiint_{V_{i}} u d V, \\
\bar{v}_{i}=\frac{1}{\Delta V_{i}} \iiint_{V_{i}} v d V, \\
\bar{s}_{i}=\frac{1}{\Delta V_{i}} \iiint_{V_{i}} s d V,
\end{gathered}
$$

Equations (3.4) and (3.5) may be rewritten as

$$
\underbrace{\frac{d \bar{u}_{i}}{d t} \Delta V_{i}}_{\text {temporal }}=\underbrace{\bar{v}_{i} \Delta V_{i}}_{\text {velocity component }}
$$




$$
\begin{aligned}
\underbrace{\frac{d \bar{v}_{i}}{d t} \Delta V_{i}}_{\text {temporal }}=- & \underbrace{\zeta \cdot \bar{v}_{i} \Delta V_{i}}_{\text {diffusiveflux }}+\underbrace{\iint_{\varsigma_{i}}(\eta \nabla v) \cdot \hat{\mathbf{n}} d \varsigma}_{\text {viscousflux }} \\
& +\underbrace{\iint_{\varsigma_{i}}\left(\nu^{2} \nabla u\right) \cdot \hat{\mathbf{n}} d \varsigma}_{\text {velocityflux }}+\underbrace{\bar{s}_{i} \Delta V_{i}}_{\text {source }} .
\end{aligned}
$$

No approximations have been made to equations (3.11) and (3.12) at this phase, thus (3.11) and (3.12) are an exact reformulation of equations (3.2) and (3.3) (Turkel, 1986). It is this formulation that generates the finite volume discretisation of (3.2) and (3.3).

\subsubsection{Discretization}

We write the equation (2.1) as a system of two first order PDEs, as in Mensah et al. (2019). Mensah et al. (2019) referred to this system of equations (3.2 and 3.3) as a velocitydisplacement diffusive-viscous wave equation. These are cell averages $\bar{u}_{i}$ and $\bar{v}_{i}$ at each control volume $\mathbf{x}_{\mathbf{i}}$, often written in vector form as

$$
\begin{aligned}
& \overline{\mathbf{u}}=\left(\bar{u}_{1}, \bar{u}_{2}, \ldots, \bar{u}_{N}\right)^{\top}, \\
& \overline{\mathbf{v}}=\left(\bar{v}_{1}, \bar{v}_{2}, \ldots, \bar{v}_{N}\right)^{\top} .
\end{aligned}
$$

The discretization of equation (2.1) depends on solving approximations of temporal, diffusive flux, viscous flux, velocity flux and source terms that include only the (unknown) variables $\bar{u}_{i}$ and $\bar{v}_{i}$, where $i=1, \ldots, N$, and $N$ is the number of control volumes. The overall accuracy of the FV method is effectively determined by the accuracy of this discretization. Time integration methods will be discussed later in this thesis.

It is worth noting that there has been some suppression of some dependencies on $u, v$ and $\mathbf{x}$ in equation (3.12) for readability sake. $\mathbf{x}=(x, y, z)$, in $3 D$ and in Cartesian coordinates. In this thesis, $\zeta$ and $\eta$ are considered to depend on $v, \nu$ is considered to depend on $u, s$ is also considered to depend on $t$, while the solution $u$ and $v$ themselves, are considered to depend on $\mathbf{x}$. Consequently, equation (3.12) could be described more 
comprehensively as

$$
\begin{array}{r}
\frac{d \bar{v}_{i}}{d t} \Delta V_{i}=-\zeta(v(\mathbf{x})) \\
\cdot \bar{v}_{i}(\mathbf{x}) \Delta V_{i}+\iint_{\varsigma_{i}}(\eta(v(\mathbf{x})) \nabla v(\mathbf{x})) \cdot \hat{\mathbf{n}} d \varsigma \\
+\iint_{\varsigma_{i}}\left(\nu^{2}(u(\mathbf{x})) \nabla u(\mathbf{x})\right) \cdot \hat{\mathbf{n}} d \varsigma+\bar{s}_{i} \Delta V_{i} .
\end{array}
$$

This type of explicit dependency will be incorporated in chapters $4,5,6$, and 7 when the complete discretization schemes in two and three dimensions are reviewed. The suppressed form of these dependencies will be maintained for now, to ease readability.

Going back to equation (3.12), if appropriate approximations for the diffusive, viscous and velocity fluxes, as well as the source term, that is, the RHS of equation (3.12), can be obtained, and if these approximations encompass only the values of $u$ and $v$ at the nodes, then equation (3.12) can be transformed into its discrete, algebraic equivalent

$$
\frac{d \bar{v}_{i}}{d t} \Delta V_{i}+f_{i}(\mathbf{u}, \mathbf{v})=0
$$

The scalar function, $f_{i}$ in equation (3.16), is represented as being dependent on the vectors $\mathbf{u}$ and $\mathbf{v}$. In reality, the interpolation scheme often guarantees that each $f_{i}$ is essentially dependent only on "local" sets of the $u_{j}$ and $v_{j}$, but generally it is however on the whole vectors $\mathbf{u}$ and $\mathbf{v}$. Every of such equation for control volume $\mathbf{x}_{\mathbf{i}}$ constitutes part of an algebraic system of equations

$$
\frac{d \bar{v}_{i}}{d t} \Delta V_{i}+\mathbf{L}(\mathbf{u}, \mathbf{v})=\mathbf{0}
$$

where

$$
\mathbf{L}=\left(f_{1}, f_{2}, f_{3}, \ldots, f_{N}\right)^{\top}
$$

represents the solution that produces the finite volume solution vectors $\mathbf{u}$ and $\mathbf{v}$. Generally, due to the dependence of the diffusivity, $\zeta$, viscosity, $\eta$ and velocity, $\nu$ on $u$ and $v$, equation (3.17) will be a non-linear system of algebraic equations.

In Sections 3.2 and 3.3, a review of the two fundamental components of the discretization of the RHS of equation (3.12) is provided: integration and interpolation. Subsequently, 
in Section 3.4, the time integration methods applied to the LHS of equation (3.12) is presented to produce the final numerical solution.

\subsection{Integration}

In order to implement equation (3.12), discrete approximations of the diffusive, viscous, and velocity fluxes, as well as the source term must be evaluated. These terms entail spatial integration of quantities whose analytical description may be arbitrarily complex or entail the unknown functions $u$ and $v$. Hence, it is justifiable to resort to numerical methods of integration in formulating these discretizations.

Firstly consider the viscous and velocity flux components

$$
\begin{gathered}
\iint_{\varsigma_{i}}(\eta \nabla v \cdot \hat{\mathbf{n}}) d \varsigma, \\
\iint_{\varsigma_{i}}\left(\nu^{2} \nabla u \cdot \hat{\mathbf{n}}\right) d \varsigma .
\end{gathered}
$$

from equation (3.12), respectively. Since $u$ and $v$ have an unknown analytical expression, correspondingly the integrands of equations (3.19) and (3.20) are unknowns. Hence, numerical methods must be relied upon to approximate them. Luckily, the control volumes, $V_{i}$, and their surfaces, $\varsigma_{i}$, are, by framework, rather easy geometric figures. Specifically, the $\mathrm{CV}$-surfaces, $\varsigma_{i}$, are comprised, in two dimensions, of line segments, and, in three dimensions, quadrilateral (see figure 3.2), allowing equations (3.19) and (3.20) to be represented as

$$
\begin{gathered}
\iint_{\varsigma_{i}}(\eta \nabla v \cdot \hat{\mathbf{n}}) d \varsigma=\sum_{\kappa=1}^{N f_{i}} \iint_{\varsigma_{i \kappa}}\left(\eta \nabla v \cdot \hat{\mathbf{n}}_{\mathbf{i k}}\right) d \varsigma, \\
\iint_{\varsigma_{i}}\left(\nu^{2} \nabla u \cdot \hat{\mathbf{n}}\right) d \varsigma=\sum_{\kappa=1}^{N f_{i}} \iint_{\varsigma_{i \kappa}}\left(\nu^{2} \nabla u \cdot \hat{\mathbf{n}}_{\mathbf{i k}}\right) d \varsigma,
\end{gathered}
$$

respectively, where $\varsigma_{i}$ is composed of $N f_{i}$ faces, $\varsigma_{i \kappa}, \kappa=1, \ldots, N f_{i}$ and $\hat{\mathbf{n}}$ is the unit normal vector to the boundary of the control volume surface. The challenge in approximating equations (3.19) and (3.20) is therefore simplified to the approximation of each 
term

$$
\begin{gathered}
\iint_{\varsigma_{i} \kappa}\left(\eta \nabla v \cdot \hat{\mathbf{n}}_{\mathbf{i k}}\right) d \varsigma, \\
\iint_{\varsigma_{i} \kappa}\left(\nu^{2} \nabla u \cdot \hat{\mathbf{n}}_{\mathbf{i k}}\right) d \varsigma,
\end{gathered}
$$

of equations (3.21) and (3.22), respectively.

\subsubsection{Midpoint rule}

A conventional approach to approximate equations (3.23) and (3.24) is to apply the midpoint rule

$$
\begin{gathered}
\iint_{\varsigma_{i \kappa}}(\eta \nabla v \cdot \hat{\mathbf{n}}) d \varsigma \\
\iint_{\varsigma_{i \kappa}}\left(\nu^{2} \nabla u \cdot \hat{\mathbf{n}}\right) d \varsigma \approx\left[\nu^{2} \nabla u \cdot \hat{\mathbf{n}}\right]_{\mathbf{m}_{\mathbf{i} \kappa}} A_{i \kappa},
\end{gathered}
$$

respectively, where $\mathbf{m}_{\mathbf{i} \kappa}$ represents the midpoint of face, $\varsigma_{i \kappa}$, and $A_{i \kappa}$ represents its area. The midpoint rule is second-order accurate.

Now consider the diffusive flux and source component on the RHS of equation 3.12. The description of these quantities in terms of the CV averaged values $\bar{v}_{i}$ and $\bar{s}_{i}$ specified in equations (3.9) and (3.10) represent the general method of approximation.

In several instances, the accuracy provided by quadrature methods in equations (3.25) and (3.26) is adequate, and the schemes are extensively implemented. However, there are certain instances where a higher order scheme of quadrature is required. Equations (3.25) and (3.26) can be considered as one-point quadrature schemes. Multiple-point quadrature schemes may, instead, be applied to attain higher accuracy.

Many prominent numerical quadrature schemes exist in one dimension which can as well be extended to implement in two and three dimensions. Typically however, there is an exponential increase in the cost of such schemes with dimension. For instance, the twopoint one dimensional quadrature method depicted in figure (3.3a) depends on four points when extended to two dimensions, and eight points when extended to three dimensions (Figures $3.3 \mathrm{~b}$ and $3.3 \mathrm{c}$ accordingly). Thus, it is useful to apply a method of quadrature 


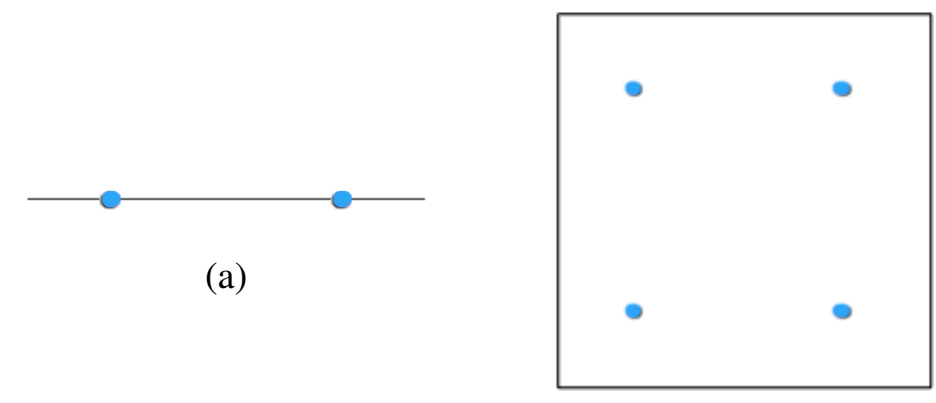

(b)



(c)

Figure 3.3: The "two-point" quadrature methods in (a) 1-D; (b) 2-D; (c) 3-D

that can obtain higher accuracy without needing an exaggerated amount of integrand estimations, since these can be significantly expensive in the finite volume approach. One such method that has been effectively applied as part of the finite volume approach is the one-point Gaussian quadrature (e.g., Ollivier-Gooch \& Van Altena, 2002; Song \& Chen, 2002) and this is the method used in this thesis.

\subsubsection{Gaussian quadrature}

A one dimensional Gaussian quadrature (Burden \& Faires, 2001; Epperson, 2013) is a technique of numerical quadrature that can be employed with an arbitrary amount of integration points or abscissa. Given a function, $f(x)$ and a weighting function, $W(x)$, over an interval $[a, b], n$-point Gaussian quadrature applies the approximation

$$
\int_{a}^{b} f(x) d x \approx \sum_{j=1}^{n} w_{j} f\left(x_{j}\right)
$$

The Gaussian weights, $w_{j}$, and the abscissas, $x_{j}$, are selected such that the approximation is exact for polynomials up to degree $2 n+1$. Particularly, the roots of the equivalent orthogonal polynomial, $P_{n}(x)$ over the interval $[a, b]$ are precisely the abscissas. Tables of Gaussian weights and abscissas for several forms of Gaussian quadrature are presented in most numerical analysis literature (e.g., see Epperson, 2013). 


\subsection{Interpolation}

In the previous section, the numerical quadrature approach for approximating equation 3.12 was discussed. The schemes reviewed in that section entail the estimation of the integrand at specific integration points. For the viscous and velocity flux components, these points are located on the control volume faces. For the diffusive flux and source term components, these points lie within the control volume itself. In the case of the viscous and velocity flux components, the integrands are dependent on $v$ and $u$ respectively.

This dependence on $u$ and $v$ is not so trivial, since when implementing the finite volume method, the points exclusively at which values of $u$ and $v$ are accessible, are the control volumes themselves. As earlier discussed in Section 3.1, the technique is based on formulating control volumes around these nodes, and computing each discretized equation, 3.12 , for the values $\bar{u}_{i}$ and $\bar{v}_{i}$.

The viscous and velocity flux components, regardless of whether they are evaluated employing the midpoint rule, higher-order Gaussian quadrature, or a different quadrature method, not only require values for $u$ and $v$, but its gradients, $\nabla u$ and $\nabla v$, as well. In this instance, some kind of interpolation must be applied that takes the cell averages $\bar{u}_{i}$ and $\bar{v}_{i}$ and generates interpolated values of $u, v, \nabla u$, and $\nabla v$ at specific positions on the $\mathrm{CV}$ surfaces.

We assume throughout this Section that all the interpolation methods discussed are based on control volumes. Particularly, each control volume in the mesh, $\xi_{i}$, has an associated interpolation function, $\tau_{i}$, that approximates $u$ and $v$ all over that control volume. Moreover, local enumeration of the control volume vertices is employed throughout, for notation simplification sake. Specifically, the vertices of $\xi_{i}$ are indicated as $\mathbf{x}_{\mathbf{1}}, \mathbf{x}_{\mathbf{2}}, \ldots, \mathbf{x}_{\mathbf{N}}$, regardless of their global position in terms of the vectors $\mathbf{u}$ and $\mathbf{v}$ (equations 3.13 and 3.14)

The objective of $\tau_{i}$ is to approximate $u$ and $v$ over the control volume $\xi_{i}$, irrespective of 
the approach applied in the design of $\tau_{i}$ :

$$
\begin{aligned}
& \tau_{i}^{u}(\mathbf{x}) \approx u(\mathbf{x}), \quad \mathbf{x} \in \xi_{i} \\
& \tau_{i}^{v}(\mathbf{x}) \approx v(\mathbf{x}), \quad \mathbf{x} \in \xi_{i}
\end{aligned}
$$

Moreover, if $\tau$ is differentiable, then the gradients, $\nabla u$ and $\nabla v$ may also be approximated using $\tau$ :

$$
\begin{aligned}
& \nabla \tau_{i}^{u}(\mathbf{x}) \approx \nabla u(\mathbf{x}), \quad \mathbf{x} \in \xi_{i}, \\
& \nabla \tau_{i}^{v}(\mathbf{x}) \approx \nabla v(\mathbf{x}), \quad \mathbf{x} \in \xi_{i} .
\end{aligned}
$$

Having now established the interpolating function, $\tau_{i}$ for $u, v$ and their gradients, all developments of $u(\mathbf{x})$ and $v(\mathbf{x})$ and their gradients, $\nabla u(\mathbf{x})$ and $\nabla v(\mathbf{x})$, all through the integration formulas in Section 3.2 may be substituted within control volume $\xi_{i}$ by $\tau_{i}^{u}$, $\tau_{i}^{v}, \nabla \tau_{i}^{u}$, and $\nabla \tau_{i}^{v}$, respectively. What is left is to determine which precise methods of interpolation might be appropriate within the finite volume scheme. Only a few of such methods will be discussed in this thesis.

\subsubsection{Function reconstruction}

In finite volume schemes, the volume averages of solution variables over local mesh cells are considered as unknowns that are integrated based on integral conservation laws with respect to time (X.-D. Liu, Osher, Chan, et al., 1994; Buchmüller, Dreher, \& Helzel, 2016; Hyman, Knapp, \& Scovel, 1992; SICOT, 2006; White \& Adcroft, 2008; Hernández, 2002). To evaluate fluxes through cell faces, a polynomial reconstruction of a local function field is usually achieved by applying cell averages, and based on the function reconstruction model, the variable values and variable gradients at cell faces are interpolated. The accuracy of a finite volume scheme is fundamentally ascertained by the accuracy of the enforced reconstruction model. To obtain a second-order accurate finite volume scheme, a linear or higher-order function reconstruction model is required to evaluate cell-face values. Some well-developed schemes are available for high-order accurate finite volume schemes, such as the WENO classification schemes (see Shu, 2003; Dumbser, 
Hidalgo, \& Zanotti, 2014; Dumbser, Zanotti, Hidalgo, \& Balsara, 2013; Titarev \& Toro, 2004; Zhou, Li, \& Shu, 2001; Xing \& Shu, 2006; Coralic \& Colonius, 2014; Noelle, Xing, \& Shu, 2007; X.-D. Liu et al., 1994; Buchmüller et al., 2016, for examples), among other (also see Toro, Hidalgo, \& Dumbser, 2009; Dumbser, Hidalgo, Castro, Parés, \& Toro, 2010; Hidalgo \& Dumbser, 2011; Hyman et al., 1992; Hernández, 2002, for examples).

\subsection{Reconstruction from cell averages. General ideas}

When applying the FV method, cell averages of the solution are obtained at each time step. Due to this, in most cases a reconstruction process must be applied. In this section, we describe the main features of the reconstruction procedure followed in this thesis. We remark that this description is quite general, while the particular reconstruction technique implemented in this thesis is based on a piecewise linear function. Here, we explained for the $1 D$ case, but it can be easily extended to $2 D$ and $3 D$ regular Cartesian meshes.

We use the notation $\left[x_{i-\frac{1}{2}}, x_{i+\frac{1}{2}}\right]$ to refer to the coordinates of the edges of the control volume $i$ whose central point has a coordinate $x_{i}$, with the index $i$ ranging from $i=1$ to $i=N$ where $N$ denotes the number of control volumes introduced during the discretization process (see Figure 3.4). It is useful to introduce a mapping using a reference coordinate, denoted here as $\Psi$, where there has been a shift of the scale and origin of the coordinate system to the center of cell, $i$, and for convenience, it has been scaled by its width such that the left and right edges are established at a scaled length of $-\frac{1}{2}$ and $\frac{1}{2}$. If the reconstruction polynomial is of certain degree $M$, it is built over $M+1$ cells. We remark that, the usual thing that is done in the FV method is to build a piecewise reconstruction function, whose restriction to each particular cell of the stencil is a polynomial. This function must be conservative, verifying that the cell average of the reconstruction function at each cell interface must be equal to the cell average of the solution. However, we act in a different way in this thesis, which consists of designing a reconstruction function which is a polynomial for each cell interface, in order to have a unique value and a unique derivative here. Going by this procedure, there are no jumps either in the solution or in the gradients at cell interfaces. We notice that this is a valid way to perform 
the reconstruction function in the context of diffusive problems, with the second derivative in space involved, but it must be avoided when dealing with first order hyperbolic problems, such as Euler equations for gas dynamics, shallow water models or the simple linear advection equation. In these situations, it is necessary to resort to the reconstruction function with jumps at cell interfaces, hence, a flux reconstruction procedure (Rusanov, Lax-Wendroff, Lax-Friedrichs, Force, ...) or the implementation of Riemann problem solvers are necessary (HLL, HLLC, Osher,...).

Let $\Psi$ be a reference variable. We define the following polynomial of degree $M$

$$
p(\Psi)=\sum_{n=0}^{M} a_{n} \Psi^{n}
$$

where $a_{n}$ are the $M+1$ coefficients to be determined from $M+1$ conditions.

We focus our attention on the right face of the control volume, denoted as $i+\frac{1}{2}$, which is the intersection of control volumes $i$ and $i+1$. We consider the $M$ candidate stencils available that contain $M+1$ cells, all of them including the control volumes enclosing the interface $i+\frac{1}{2}$, that is $i$ and $i+1$. These stencils are

$$
S_{\alpha}=\{i+\alpha, i+\alpha+1, \cdots \ldots, i+\alpha+M\},(\alpha=-M+1,-M+2, \cdots, 0)
$$

In order to get the expression of such polynomial we have to apply the conservation property on each particular control volume of the stencil and considering that, in reference coordinates, the length of each cell is the unity, and the following systems of equations arises

$$
C_{j, l}^{(\alpha)} a_{l}=\bar{F}_{i+j+\alpha}(j=0, \cdots, M ; l=0, \cdots, M)
$$

where the elements of the matrix $\mathbf{C}^{(\alpha)}$, for each particular stencil $S_{\alpha}$ can be computed as

$$
C_{j, l}^{(\alpha)}=\int_{j+h}^{j+h+1} \Psi^{l} d \Psi(j=0, \cdots, M ; l=0, \cdots, M)
$$

with $h=\alpha-\frac{1}{2}, \alpha=-M+1,-M+2, \cdots, 0$. Hence, we have $M$ matrices of the 
form (3.35) with each of them for a particular candidate stencil, $S_{\alpha}$. After performing the integration in (3.35) we attain

$$
C_{j, l}^{(\alpha)}=\frac{1}{l+1}\left((j+h+1)^{l+1}-(j+h)^{l+1}\right)(j=0, \cdots, M ; l=0, \cdots, M)
$$

Proceeding in this way, we obtain $M$ different values of the reconstruction function at the interface $x_{i+\frac{1}{2}}$. We can use some kind of weighted averaging to have a unique value there. In usual weighted averaging processes, the less biased stencils receive a larger weight, for stability reasons.

For clarity sake, we are going to consider the particular case where $M=3$, that is a third degree piecewise polynomial function. In this case, we have three candidate stencils $S_{\alpha}$ for the values $\alpha=-2,-1,0$, each one with four cells. These stencils, according to the formula (3.33) are $\{i-2, i-1, i, i+1\},\{i-1, i, i+1, i+2\}$ and $\{i, i+1, i+2, i+3\}$. Hence, for each particular stencil $S_{\alpha}$, we apply the conditions given in (3.34) with the coefficients (3.36) getting, for the matrices of coefficients, $\mathbf{C}^{(\alpha)}$, and the RHS vector, $\mathbf{F}^{(\alpha)}$

- For $\alpha=-2$, that is stencil $\{i-2, i-1, i, i+1\}$

$$
\mathbf{C}^{(-2)}=\left(\begin{array}{cccc}
1 & -2 & \frac{49}{12} & -\frac{17}{2} \\
1 & -1 & \frac{13}{12} & -\frac{5}{4} \\
1 & 0 & \frac{1}{12} & 0 \\
1 & 1 & \frac{13}{12} & \frac{5}{4}
\end{array}\right) ; \mathbf{F}^{(-2)}=\left(\begin{array}{c}
\bar{F}_{i-2} \\
\bar{F}_{i-1} \\
\bar{F}_{i} \\
\bar{F}_{i+1}
\end{array}\right)
$$

- For $\alpha=-1$, that is $\{i-1, i, i+1, i+2\}$

$$
\mathbf{C}^{(-1)}=\left(\begin{array}{cccc}
1 & -1 & \frac{13}{12} & -\frac{5}{4} \\
1 & 0 & \frac{1}{12} & 0 \\
1 & 1 & \frac{13}{12} & \frac{5}{4} \\
1 & 2 & \frac{49}{12} & \frac{17}{2}
\end{array}\right) ; \mathbf{F}^{(-1)}=\left(\begin{array}{c}
\bar{F}_{i-1} \\
\bar{F}_{i} \\
\bar{F}_{i+1} \\
\bar{F}_{i+2}
\end{array}\right)
$$

- For $\alpha=0$, that is $\{i, i+1, i+2, i+3\}$ 


$$
\begin{aligned}
& \mathbf{C}^{(0)}=\left(\begin{array}{cccc}
1 & 0 & \frac{1}{12} & 0 \\
1 & 1 & \frac{13}{12} & \frac{5}{4} \\
1 & 2 & \frac{49}{12} & \frac{17}{2} \\
1 & 3 & \frac{109}{12} & \frac{111}{4}
\end{array}\right) ; \mathbf{F}^{(0)}=\left(\begin{array}{c}
\bar{F}_{i} \\
\bar{F}_{i+1} \\
\bar{F}_{i+2} \\
\bar{F}_{i+3}
\end{array}\right)
\end{aligned}
$$

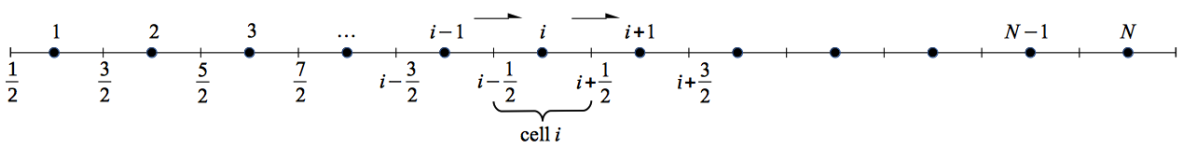

Figure 3.4: Discretization of a one-dimensional domain into computational cells(grid) of width, $\delta x$. The cell centers are illustrated by dark-filled circles and the vertical lines indicate the cell edges; the cell number is illustrated above the cells while the cell edges are indicated by the numbers below.

Filling the entries of the matrix now becomes straightforward since

$$
\begin{aligned}
A_{j, n} & =\int_{x_{j-\frac{1}{2}}}^{x_{j+\frac{1}{2}}}\left(\frac{x-x_{i-\frac{1}{2}}}{\delta x_{i}}\right)^{n-1} \mathrm{~d} x, \quad \text { for } n=1,2, \ldots, N+1 \\
& =\frac{\delta x_{i}}{n}\left[\left(\frac{x_{j+\frac{1}{2}}-x_{i-\frac{1}{2}}}{\delta x_{i}}\right)^{n}-\left(\frac{x_{j-\frac{1}{2}}-x_{i-\frac{1}{2}}}{\delta x_{i}}\right)^{n}\right]
\end{aligned}
$$

\subsubsection{Piecewise constant reconstruction}

The simplest possible reconstruction algorithm is to consider the function to be constant within each cell (suggesting that the conserved variables are as well constant) as illustrated in figure 3.5. The approximation of the function becomes

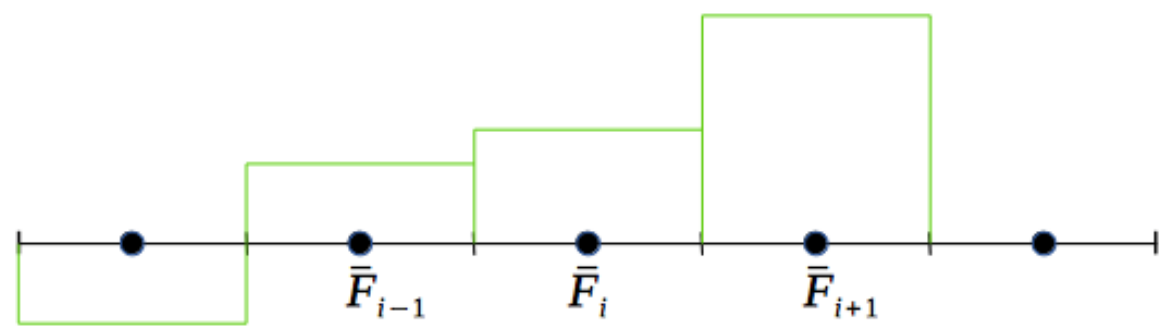

Figure 3.5: Piecewise Constant Approximation.

$$
F=a_{0}
$$


Only a single constraint can be imposed, after all there is only one unknown coefficient, $a_{0}$, indicating that the integral of $F$ over cell $i$ yields

$$
\int_{i-\frac{1}{2}}^{i+\frac{1}{2}} a_{0} \mathrm{~d} x=\delta x_{i} \bar{F}_{i}
$$

The solution is then naturally $a_{0}=\bar{F}_{i}$. The reconstruction of the function at $x_{i+\frac{1}{2}}$ can be accomplished. A few points are worthy of note regarding the implementation of the solution algorithm, and they are that:

- It is possible to carry out two approximations at the edge point, that is, one at the left cell, $i$, and another at the right cell, $i+1$. The physical intuitive justification is that the flux arriving at the cell edges must arrive from the so-called upstream cell (the cell in the flux propagation direction). Hence we have

$$
F_{i+\frac{1}{2}}= \begin{cases}\bar{F}_{i} & \text { if } u_{i+\frac{1}{2}} \geq 0 \\ \bar{F}_{i+1} & \text { if } u_{i+\frac{1}{2}}<0\end{cases}
$$

- Piecewise constant functions tend to have a zero spatial derivation, and therefore piecewise constants are ineffective in evaluating the function derivation for any order of derivation.

The piecewise constant functions lead to a first-order reconstruction which is significantly highly diffusive for applications. Nevertheless, it is appropriate for testing purposes only in those situations where accuracy is not necessarily required.

\subsubsection{Piecewise linear reconstruction}

We consider the particular case of piecewise linear reconstruction (see figure 3.6). Its restriction to the cell interface $i+\frac{1}{2}$ is a first degree polynomial given by

$$
p(x)=\bar{F}_{i}+s\left(x-x_{i}\right)
$$


where $s$ is the slope of the straight line defining such polynomial whose value is

$$
s=\frac{2\left(\bar{F}_{i+1}-\bar{F}_{i}\right)}{\delta x_{i}+\delta x_{i+1}}\left(x-x_{i}\right)
$$

where $x_{i}=x_{i+\frac{1}{2}}+\frac{\delta x_{i}}{2}$. The polynomial defined by the formula (3.45) is conservative in the control volumes that share the interface $x_{i+\frac{1}{2}}$, hence it verifies the conditions

$$
\begin{aligned}
& \frac{1}{\delta x_{i}} \int_{x_{i-\frac{1}{2}}}^{x_{i+\frac{1}{2}}} p(x) d x=\bar{F}_{i} \\
& \frac{1}{\delta x_{i+1}} \int_{x_{i+\frac{1}{2}}}^{x_{i+\frac{3}{2}}} p(x) d x=\bar{F}_{i+1}
\end{aligned}
$$

If we carry out the change of variable proposed in the previous section, that is $\Psi=\frac{x-x_{i}}{\delta x_{i}}$, where again $x_{i}=x_{i+\frac{1}{2}}+\frac{\delta x_{i}}{2}$ the expression of the polynomial reads

$$
p(x)=\bar{F}_{i}+\left(\bar{F}_{i+1}-\bar{F}_{i}\right) \Psi
$$

According to the transformation followed, the control volumes $\left[i-\frac{1}{2}, i+\frac{1}{2}\right]$ and $\left[i+\frac{1}{2}, i+\frac{3}{2}\right]$ converts into $\left[-\frac{1}{2}, \frac{1}{2}\right]$ and $\left[\frac{1}{2}, \frac{3}{2}\right]$ respectively. The approximation is then given as

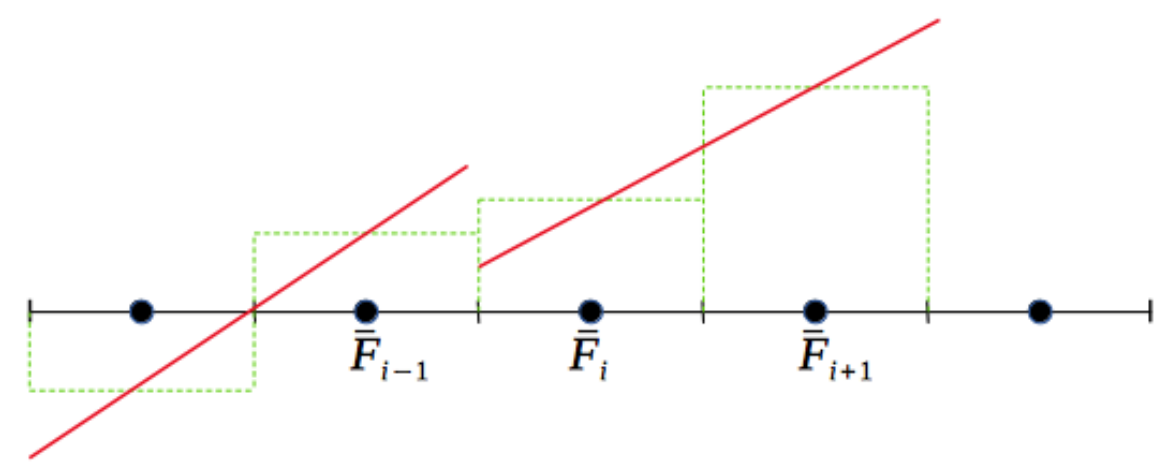

Figure 3.6: Piecewise Linear Approximation (Red lines). The green-dashed lines are cell averages given by a piecewise constant function.

$$
F=a_{0}+a_{1} \Psi
$$


The piecewise linear reconstruction leads to a second-order accurate scheme. The piecewise linear reconstruction scheme is implemented in this thesis and will further be reviewed in two and three dimensions in subsequent Chapters.

\subsubsection{Piecewise parabolic reconstruction}

It has been established that applying third-order accurate spatial reconstruction, such as the piecewise parabolic reconstruction, has the ability of lowering the amplitude truncation error whilst increasing the accuracy of the numerical solution (Harten, Lax, \& Leer, 1983; Toro, 2013; Van Leer, 2006; Stone, Gardiner, Teuben, Hawley, \& Simon, 2008). The polynomial of a parabolic interpolation centered on the cells, $i-1, i$, and $i+1$, will

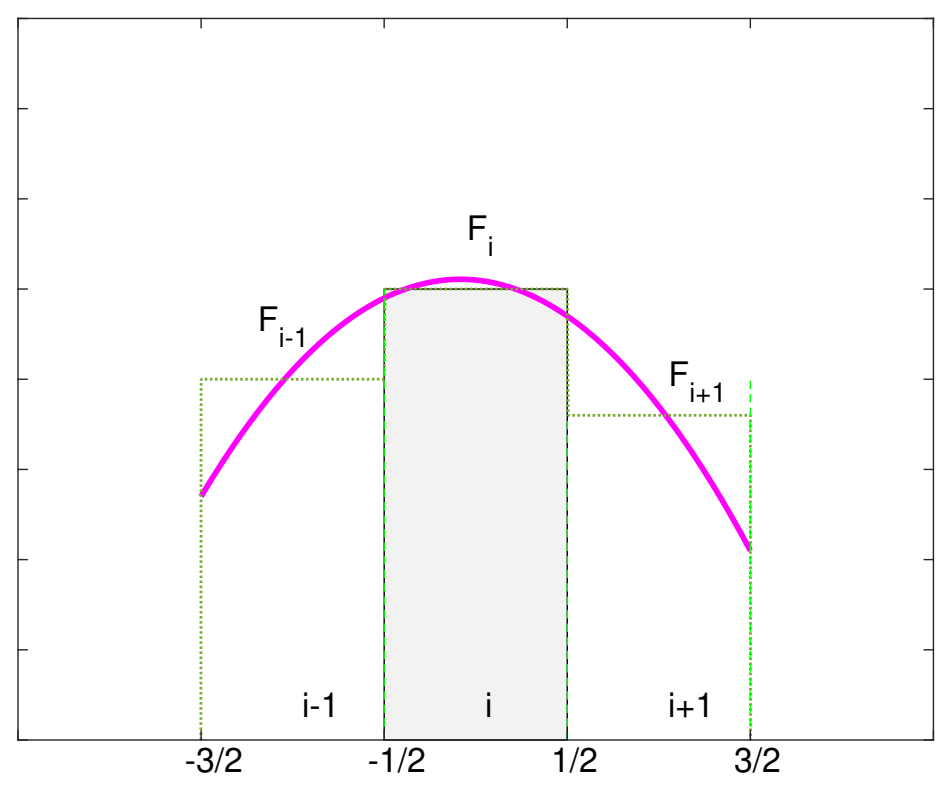

Figure 3.7: Piecewise Parabolic Approximation (Purple lines). The green-dashed lines are cell averages given by a piecewise constant function..

take the form

$$
\begin{aligned}
F= & {\left[\frac{-\bar{F}_{i+1}+26 \bar{F}_{i}-\bar{F}_{i-1}}{24}\right]+\left[\frac{\bar{F}_{i+1}-\bar{F}_{i-1}}{2}\right] \Psi+\left[\frac{\bar{F}_{i+1}-2 \bar{F}_{i}+\bar{F}_{i-1}}{2}\right] \Psi^{2}, } \\
& \text { for }-\frac{3}{2} \leq \Psi \leq \frac{3}{2}, \quad \text { where } \Psi=\frac{x-x_{i}}{\delta x}
\end{aligned}
$$


The value at the cell edge, $x_{i+\frac{1}{2}}$, can now be evaluated by substituting $\Psi=\frac{1}{2}$ into equation 3.50 above to obtain

$$
F_{i+\frac{1}{2}}=\frac{-\bar{F}_{i-1}+5 \bar{F}_{i}+2 \bar{F}_{i+1}}{6}
$$

This type of reconstruction is third order since you consider a fixed stencil. If you apply WENO methodology, which is a nonlinear reconstruction, the order of accuracy is five. Refer, for instance, to Shu (2003); Titarev and Toro (2004).

\subsubsection{Shape functions}

The finite volume method borrows a famous method of interpolation from the finite element concept (Bailey, Taylor, Cross, \& Chow, 1999; F. Liu, Turner, \& Anh, 2002; Ferguson \& Turner, 1996; Pasdunkorale Arachchige, 2003; A. Jayantha, 2001; Patankar, 1980). The shape function technique employs the vertices of a triangle, or tetrahedral element, to estimate a linear interpolation of $u$ and $v$, fulfilling

$$
\begin{aligned}
& \tau_{i}^{u}\left(\mathbf{x}_{j}\right) \approx u_{j}, \quad j=1, \ldots, m, \\
& \tau_{i}^{v}\left(\mathbf{x}_{j}\right) \approx v_{j}, \quad j=1, \ldots, m,
\end{aligned}
$$

where $m$ represents the number of vertices of the element. When $m$ is three, it represent two-dimensional, triangular elements and when $m$ is four, the elements are threedimensional, tetrahedral. Because $\tau_{i}$ is linear, the shape function interpolants for element, $\xi_{i}$ are uniquely determined by equations 3.52 and 3.53 .

In the finite element theory, the interpolant, $\tau_{i}$ is generally described as a linear combination of so-called shape functions, $Q_{j}(\mathbf{x})$ (Cook et al., 2007):

$$
\begin{array}{r}
\tau_{i}^{u}(\mathbf{x})=\sum_{j=1}^{m} u_{j} Q_{j}(\mathbf{x}), \\
\tau_{i}^{v}(\mathbf{x})=\sum_{j=1}^{m} v_{j} Q_{j}(\mathbf{x}),
\end{array}
$$

where $Q_{j}$ is only dependent on the geometric characteristics of element, $\xi_{i}$, and not on 
the values, $u_{j}$ and $v_{j}$. Formulas for $Q_{j}$ in two and three dimensions are provided in subsequent chapters.

Shape function interpolations as well permit the approximation of the gradients, with $\nabla \tau_{i}^{u}$ and $\nabla \tau_{i}^{v}$ given by

$$
\begin{aligned}
& \nabla \tau_{i}^{u}(\mathbf{x})=\sum_{j=1}^{m} u_{j} \nabla Q_{j}(\mathbf{x}), \\
& \nabla \tau_{i}^{v}(\mathbf{x})=\sum_{j=1}^{m} v_{j} \nabla Q_{j}(\mathbf{x}),
\end{aligned}
$$

which is constant all through the element.

Finite volume schemes that utilize the shape functions approach as a method of interpolation are usually referred to as the control volume-finite element methods (CV-FE). The most apparent disadvantage of the method is the application of a constant gradient approximation within every element. For problems where there is no significant variation in the gradients, this method can be sufficient. Nevertheless, in various problems involving regions with significant gradient variation, applying a constant gradient approximation may not be sufficient representing this behaviour (Pasdunkorale Arachchige, 2003; A. Jayantha, 2001).

In such instances, mesh refinement is the only option to enhance the accuracy of this method, which can be computationally expensive in relation to computational time and memory requirements. What is ideally required in such instances is a method of interpolation, more sophisticated to provide accurate function and gradient approximations even on course mesh.

\subsubsection{Assuring flux consistency}

The conservative nature of the finite volume method remains its main advantage over other numerical method like the finite element and finite difference methods. Nearly all real-world applications of partial differential equations (PDEs) are developed from fundamental physical conservation laws. Omitting the temporal components of equations 3.11 and 3.12, the RHS of 3.11 and 3.12 represents the stead-state DVWE. Without the 
LHS, equations 3.11 and 3.12 are mathematical statements indicating that, at steady state, the flux propagating through the surface of any control volume is equivalent to the net output within it. The finite volume method will maintain this conservation law, possibly at the discrete level, if formulated correctly.

The discretization method must guarantee the consistency of the flow through any two neighbouring control volume faces for this to be so. In particular, the flow measured, through the adjacent control volume faces, from the current control volume to the next must equal the negative of the flow as measured from the next control volume to the current.

Figure 3.8 depicts how this condition is fulfilled when applying grid-wise interpolation. The flux between the two adjacent control volumes (on the control volume faces) is estimated as an integral over their mutual control volume faces. All interpolated values of $u$ and $v$ and their gradients, $\nabla u$ and $\nabla v$ are equal, irrespective of the orientation with which they are computed. The rationale for this is that the points computed on either side of the control volume face, lie inside the same grid (control volume) and as such the same interpolant is used.

\subsection{Time integration}

For time dependent problems such as equations 3.11 and 3.12, integration must be performed in time as well. Time stepping schemes may be categorized into explicit and implicit schemes. Explicit schemes are straightforward, since no system of equations are solved, and they allow for an arbitrary order accuracy in time. The limitation of the explicit methods is that they often require smaller time steps, usually much smaller than is needed for reasons related to the accuracy, due to stability concerns. As such, they are inefficient in dealing with various practical computations. Conversely, implicit methods tend to be unconditionally stable and permit arbitrary time stepping, which is only controlled by the accuracy conditions. In this thesis, the time stepping method employed is the second-order Runge-Kutta TVD method (Gottlieb \& Shu, 1998; Shu \& Osher, 1988). 


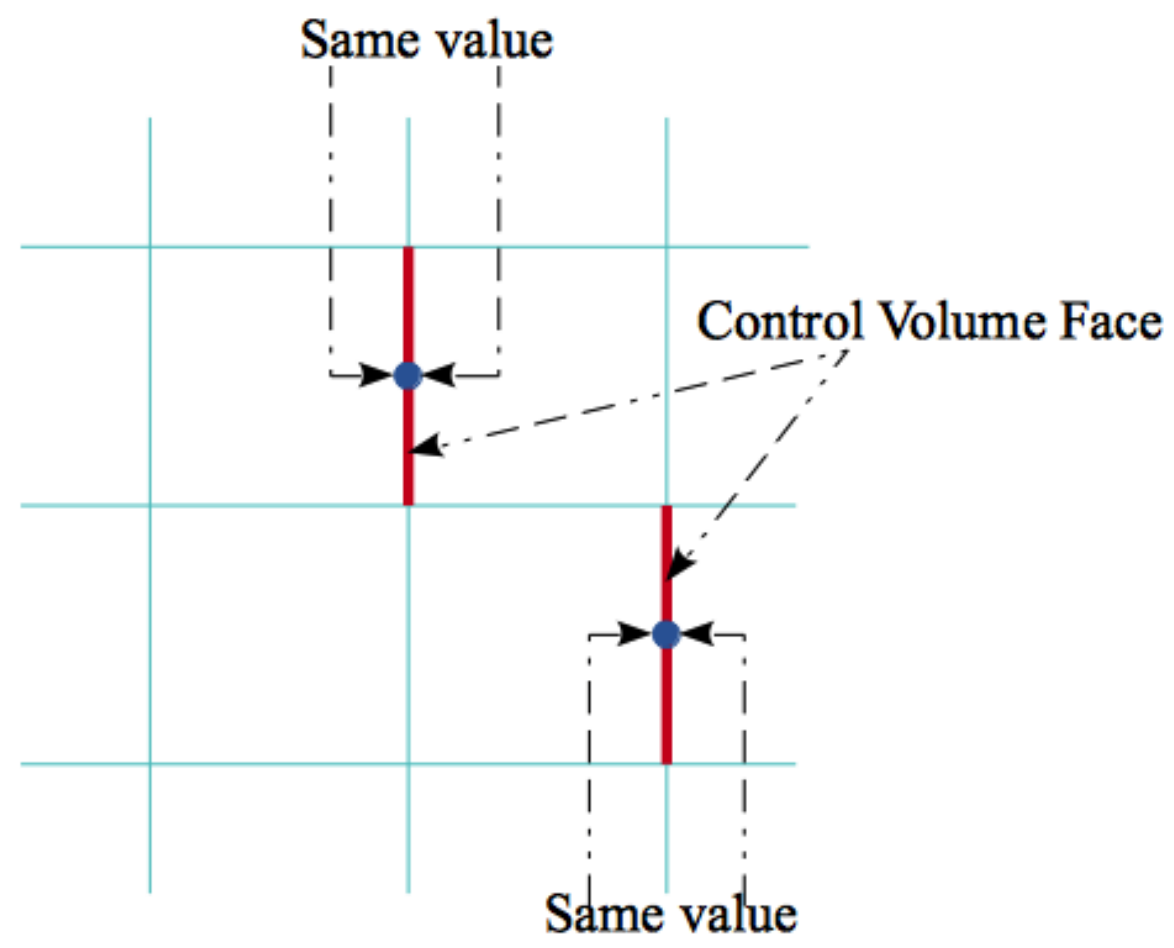

Figure 3.8: Computing fluxes using grid-wise interpolation. Red lines represent the control volume faces.

\subsubsection{Runge-Kutta TVD time integration}

A higher-order scheme is required, in some instances, to improve the accuracy of the numerical scheme (see Mensah et al., 2019; Hidalgo \& Dumbser, 2011; Toro et al., 2009; Dumbser et al., 2010, 2014, 2013; Lilek, Muzaferija, Perić, \& Seidl, 1997; VázquezCendón, Hidalgo, Navarro, \& Cea, 2012; de la Puente et al., 2007, 2008, 2009). In this thesis, the second-order Runge-Kutta TVD scheme is implemented to improve upon the accuracy of our numerical scheme. In the explicit Euler method, the slope information or derivatives at a given time step were used to extrapolate the solution to the next time step. Runge-Kutta schemes are a class of schemes which carefully applies the 'slope' information at multiple points to extrapolate the solution to the future time level. The expression for the second-order Runge-Kutta TVD method (Shu \& Osher, 1988), required 
to integrate the LHS of equations 3.11 and 3.12 is given by

$$
\begin{aligned}
Q_{P}^{(1)} & =Q_{P}^{n}+\Delta t \cdot \mathbf{L}_{P}\left(Q_{P}^{n}\right), \\
Q_{P}^{n+1} & =\frac{1}{2} Q_{P}^{n}+\frac{1}{2} Q_{P}^{(1)}+\frac{1}{2} \Delta t \cdot \mathbf{L}_{P}\left(Q_{P}^{(1)}\right),
\end{aligned}
$$

where $\mathbf{Q}_{P}$ represents the cell average of the vector of unknowns $\mathbf{Q}$ in the control volume, $P$ and $\mathbf{L}_{P}(u(t), v(t))$ represents the cell average of the operator appearing on the RHS of equations 3.11 and 3.12 over the control volume, $P$. It is worth mentioning that, to implement the second-order Runge-Kutta TVD scheme, the temporal component (LHS) of equations 3.11 and 3.12 are maintained as ODEs. The local truncation error for this method is $\mathcal{O}\left(h^{3}\right)$. Refer to Gottlieb \& Shu, 1998; Shu \& Osher, 1988, for further details on Runge-Kutta TVD schemes.

\subsection{Summary}

In this chapter, we have presented an overview of the finite volume implementation of the DVWE. Further in this chapter, various spatial discretization techniques to the DVWE have also been reviewed. These included several integration and interpolation methods such as midpoint, Gaussian quadrature and shape function methods. In additions, we have discussed the time integration schemes applied to the DVWE, which are, the RungeKutta TVD schemes. 


\section{Chapter 4}

\section{Two-dimensional considerations}

In this chapter, we present details of applying the finite volume method in two dimensions. It is worth noting that three-dimensional terminologies such as "volume", "face", and "area" will usually be employed when inferring to the corresponding two-dimensional notations of "length", "edge", and "area". The aim of this is to establish uniformity with the review in Chapter three, and since these are standard terminologies in the finite volume literature. Conversely, mathematical equations will always be written in two-dimensional notations whenever they are presented. It is also worth mentioning that the term "element" and "grid" will be used interchangeably.

\subsection{Meshing}

In Section 3.1, we discussed the mesh-based characteristics of the finite volume method. There are several alternatives of connecting a set of nodes to design the control volumes of a mesh. Common mesh types in two-dimensions can be categorized into structured and unstructured meshes (figure 4.1). At least one of these two categories of meshes can be generated by several mesh generation softwares. Less common is the capability to produce mixed meshes, whereby a combination of different control volume categories are applied. In this chapter, the premise is that all meshes are structured, even though one could easily generalize the concepts.

A structured mesh is designed with a regular grid of control volumes. An example is presented in figure 4.1a. Structural meshes tend to be most beneficial when the fundamental 

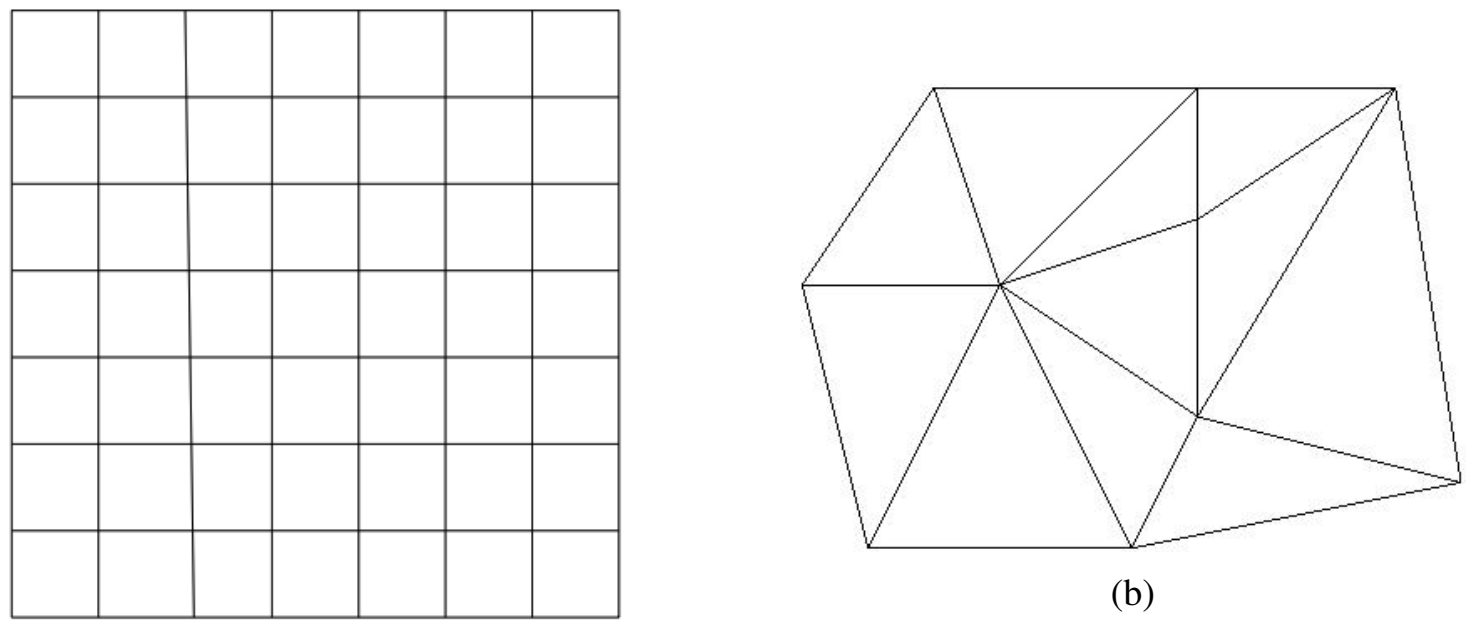

(b)

(a)

Figure 4.1: Finite volume meshes: (a) structured; (b) unstructured.

domain is made up of simple geometric regions that are adaptable to a regular grid. Certain numerical PDE solution techniques, for instance, the finite difference method, are almost particularly implemented over structured meshes. These methods can be laborious to implement, for problems where structured mesh generation is cumbersome.

Alternatively, an unstructured mesh can be applied, where there is no underlying grid. An example is presented in figure $4.1 \mathrm{~b}$. Unstructured meshes have the ability to closely fit complex and irregular boundaries, which are usually experienced in real world problems due to the freedom it offers. Furthermore, it allows for mesh refinement in regions where the fundamental physical problem requires careful scrutiny.

There is an additional complexity in the cost of implementing unstructured meshes. These additional complexities result from both the mesh generation software and the mesh-based PDE solver. It is often tedious to generate an unstructured mesh of good quality, and it is absolutely a task best left to dedicated software.

\subsection{Designing control volumes}

Finite volume discretization depends on designing control volumes (CVs) around each individual node of a mesh. There are various possibilities of specifying the shape and 




(a)

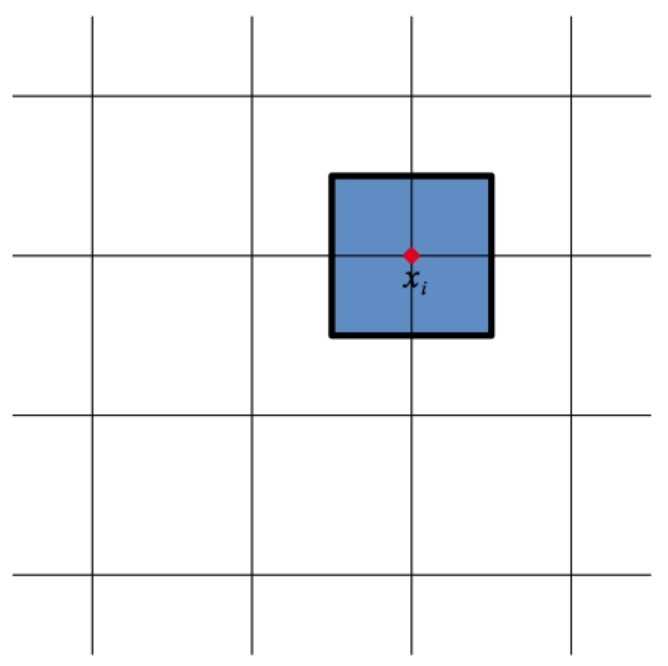

(b)

Figure 4.2: Designing control volumes: (a) cell-centered control volume; (b) vertexcentered control volume.

position of the control volume corresponding to the grid. In this section, an overview of the construction of $\mathrm{CVs}$ is presented.

Figure 4.2 depicts a section of a mesh, with two different construction methods of control volumes on a Cartesian mesh. In figure $4.2 \mathrm{a}$, the vertices of an element have been connected to each other to form a control volume whose centroid is located exactly in the middle of this element. The connection of the element vertices forms the control volume faces. The solutions are stored at the centroids of the grid cells. Hence the control volumes are equivalent to the grid cells. In figure $4.2 \mathrm{~b}$, the control volume is formed around the vertex of an element, with that vertex representing the centroid of the control volume. The control volume faces are located within the elements. The solutions are stored at the element vertices or grid points themselves. The control volume can thus, either represent the union of all cells that share the element vertex (grid point), or some volume centered around the element vertex. In the former scenario we refer to overlapping control volumes, and in the latter scenario we refer to dual control volumes. 


\subsubsection{Sub-control volumes (SCV)}

The sum of the inputs from every element that shares the node of a control volume defines the volume of a control volume. Each of these inputs is described as a sub-control volume (SCV). Figure 4.5 illustrates a control volume composed of four sub-control volumes.

An element (grid) can as well be considered as being made up of sub-control volumes. However, the number of sub-control volumes comprising a quadrilateral element does not vary, unlike in the case of control volumes: it is always four in number. Figure 4.5a shows a control volume with four sub-control volumes. Figure $4.5 \mathrm{~b}$ also illustrates a single subcontrol volume.

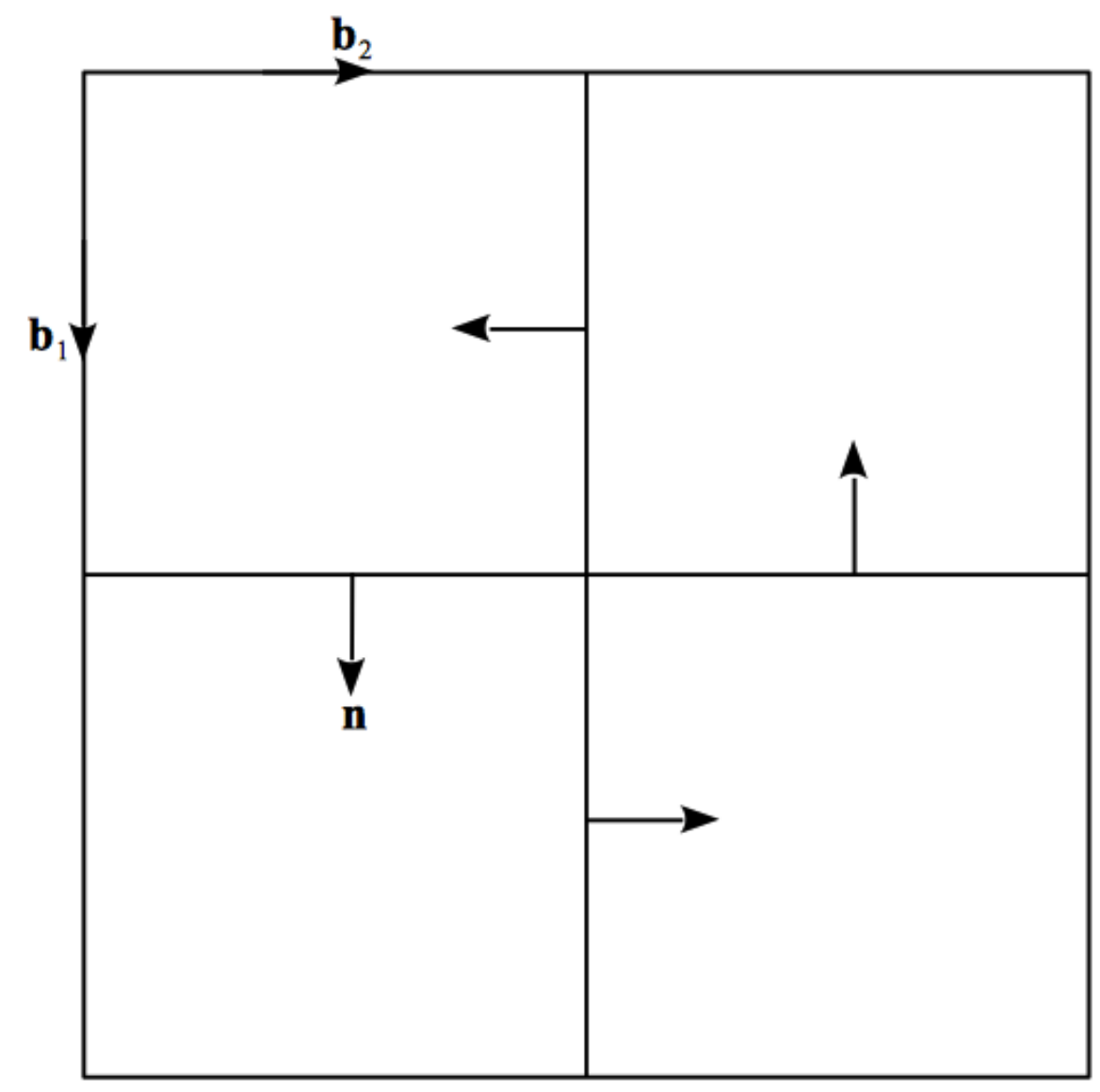

Figure 4.3: Correct, anticlockwise ordering.

It is significant to take into consideration, the four faces of a SCV as being split into pairs. The first pair of faces are those that are internal to a non-boundary control volume, and are 


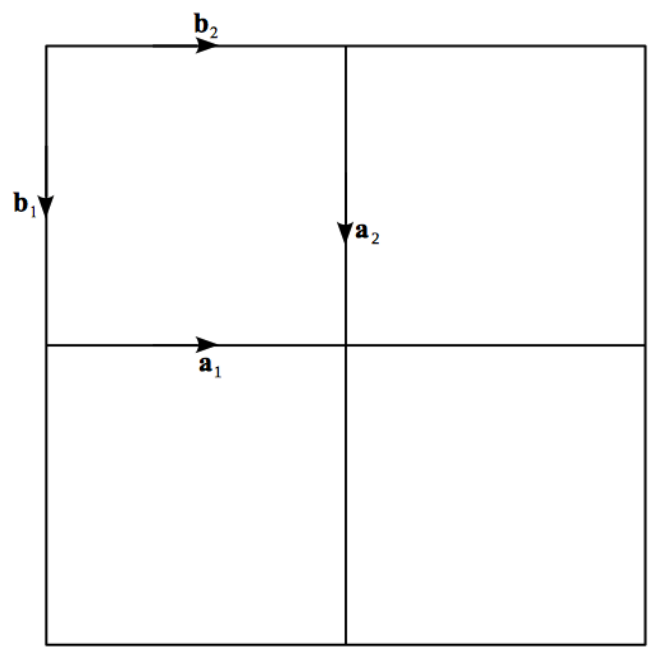

(a)

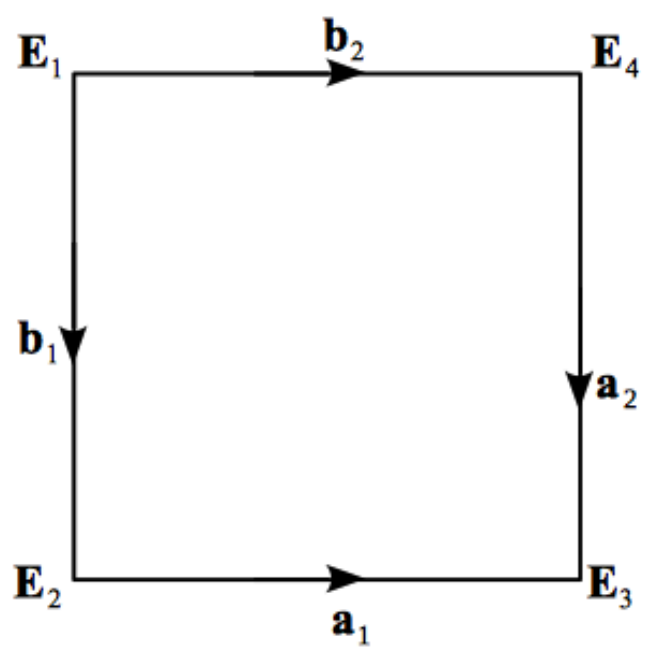

(b)

Figure 4.4: A sub-control volume (a) as one of four comprising a control volume; (b) on its own.

referred to as "inner faces" in this thesis. They are indicated in figure 4.4 by the vectors $\mathbf{b}_{1}$ and $\mathbf{b}_{2}$, with the convention that these vectors point away from the control volume vertex. Figures 4.3 and $4.5 \mathrm{~b}$ illustrate the surface normal vectors as well as their orientation of propagation, with the convention that these vectors point outwards and away from the vertex of the element. The second pair of faces, which form part of each control volume surface, is referred to as the "outer faces" in this thesis. They are indicated in figure 4.4 by the vectors $\mathbf{a}_{1}$ and $\mathbf{a}_{2}$, with the convention that these vectors point to the control volume centroid. The outward unit normal vector is expressed as:

$$
\hat{\mathbf{n}}=\mathbf{n}_{\mathbf{x}} \mathbf{i}+\mathbf{n}_{\mathbf{y}} \mathbf{j}
$$

Here $\mathbf{n}_{\mathbf{x}}$ and $\mathbf{n}_{\mathbf{y}}$ represents vectors of the outer faces. The numbering of the $\mathbf{a}_{i}$ and $\mathbf{b}_{i}$ is such that the vector

$$
\mathbf{n}=\left(\mathbf{a}_{\mathbf{1}} \mathbf{j}\right) \mathbf{i}-\left(\mathbf{a}_{\mathbf{1}} \mathbf{i}\right) \mathbf{j}
$$

when positioned as illustrated in figures 4.3 and $4.5 \mathrm{~b}$ (black circle with arrowhead), and points anticlockwise around the control volume. It is worth noting that $\mathbf{n}$ is the outward normal vector, $\hat{\mathbf{n}}$ is the outward unit normal vector, $\mathbf{a}_{i}$ is the control volume outer face vector, and $\mathbf{b}_{i}$ is the control volume inner face vector. 


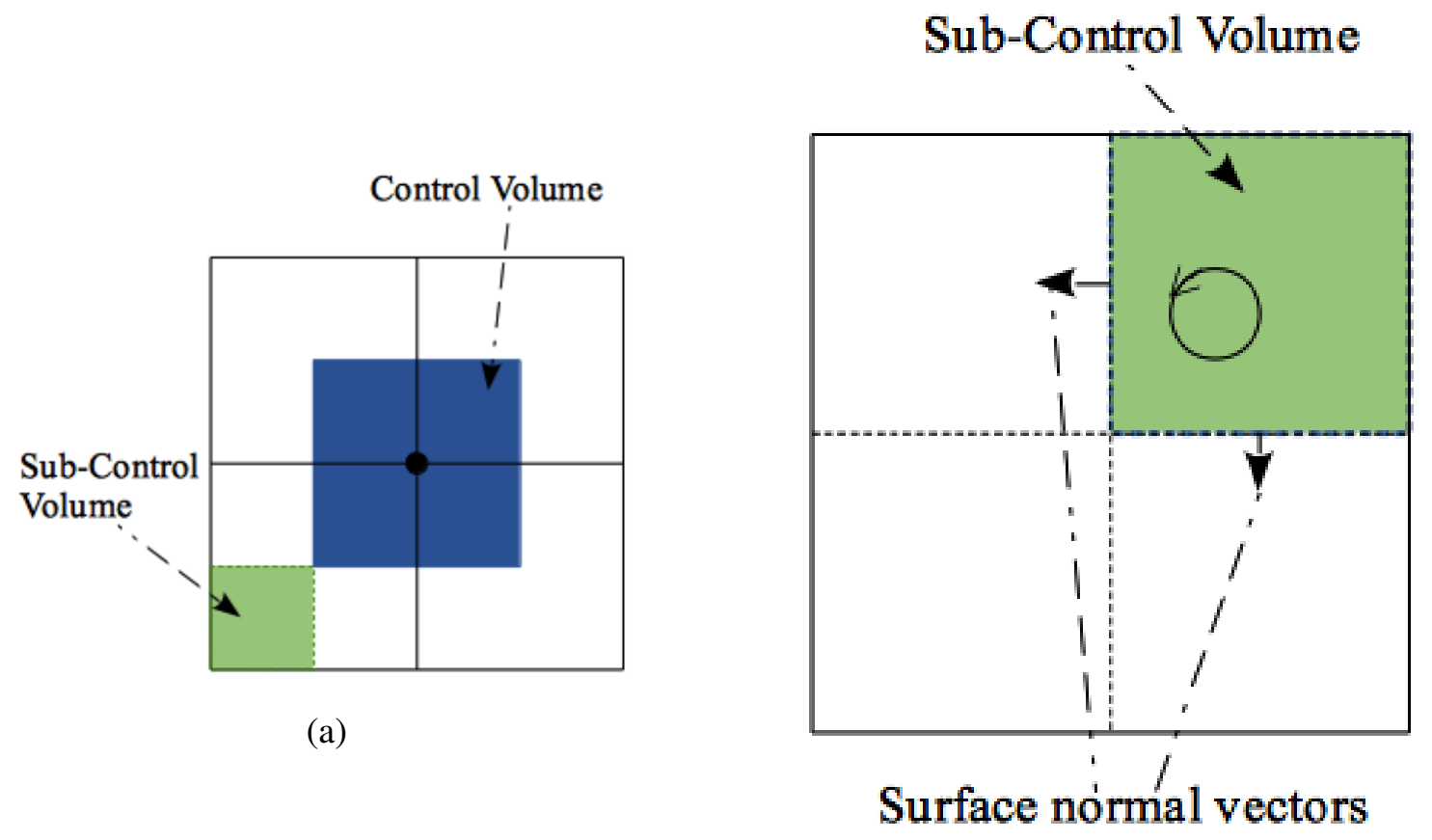

(b)

Figure 4.5: A sub-control volume (a) with a control volume; (b) on its own.

\subsection{Conventions}

In Section 3.3.2, we reviewed the fundamental conservation laws that govern several physical processes that are modelled by PDEs. It is worth recalling from that discussion that, the fluxes evaluated through the control volume faces must be consistent in both directions for the laws of conservation to be valid at the discrete level. In this section, the twodimensional implementations to ensure this consistency are reviewed, as well as other numbering conventions.

A square or rectangular control volume (grid) consists of four nodes and four faces. A simple numbering convention is applied for easy correlation of nodes with corresponding faces. Each node and control volume face is numbered from 1 to 4, in an anti-clockwise direction as illustrated in figure 4.6. Each control volume has four nodes, with two degrees of freedom per node, that is, each node can move in the $x-$ and $y$ - directions only. This implies that, every control volume has eight degrees of freedom. Face 1 corresponds to nodes 1 and 2, face 2 corresponds to nodes 2 and 3, and so on. It is worthy of remark 
that this is a local numbering arrangement peculiar to a particular control volume. On a global scale, each node and face within the mesh has a distinct identifier, with no specific connection between them.

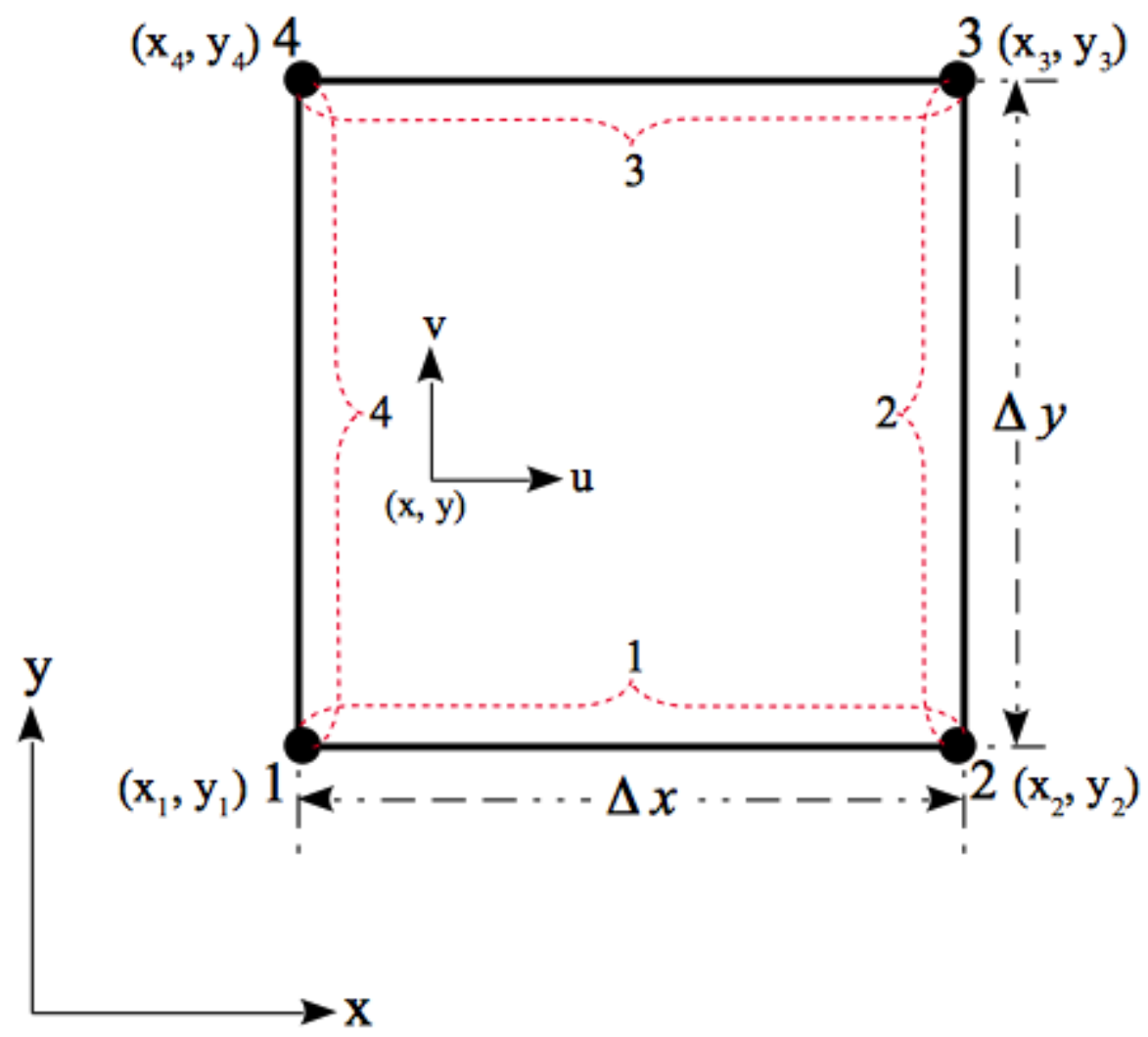

Figure 4.6: Numbering convention for nodes and control volume faces.

A grid-wise (control volume-wise) processing of the entire mesh is implemented in this thesis for the finite volume method. The flux propagating through each control volume face, within a given control volume, is computed only once, in the normal plane, $\mathbf{n}$. This common value is used in updating control volumes sharing that face, with the right modification in the sign.

Supplementary fluxes should be processed for boundary control volumes. Figure 4.7 illustrates the supplementary normal (gold arrows) on the "inner" control volume faces for a specific boundary control volume (boundary grid). These inner control volume faces are only processed within the boundary control volumes. The inner faces, as the name implies, are internal to the control volumes, therefore fluxes that flow through these 


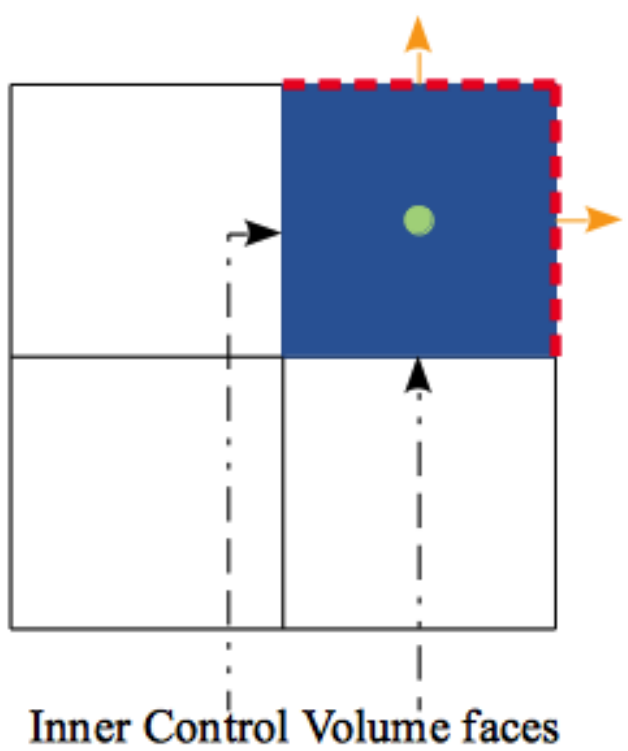

(a)

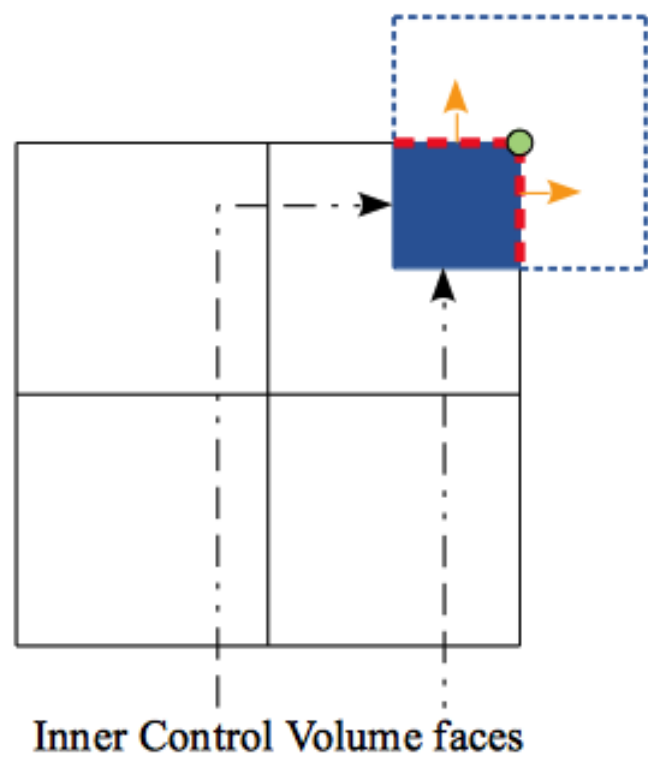

(b)

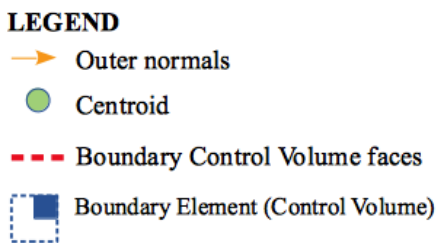

Figure 4.7: Outer normal for a boundary element: (a) cell-centered boundary element; (b) vertex-centered boundary element

faces must not be accounted for in non-boundary control volumes.

\subsection{Integration}

This Section focuses on the two dimensional application of the integration methods reviewed in Section 3.2. In equations 3.21 and 3.22, the integral over a control volume surface is given as the sum of integrals over each control volume face. These integrals are more aptly represented as line integrals, in two dimensions (refer to figure 4.8):

$$
\begin{aligned}
\int_{C_{i}}(\eta \nabla v \cdot \hat{\mathbf{n}}) d s & =\sum_{\kappa=1}^{N f_{i}} \int_{C_{i \kappa}}(\eta \nabla v \cdot \hat{\mathbf{n}}) d s \\
& =\sum_{\kappa=1}^{N f_{i}} \int_{\mathbf{x}_{\mathbf{i} \kappa}}^{\mathbf{x}_{\mathbf{i} \kappa+1}}(\eta \nabla v \cdot \hat{\mathbf{n}}) d s
\end{aligned}
$$




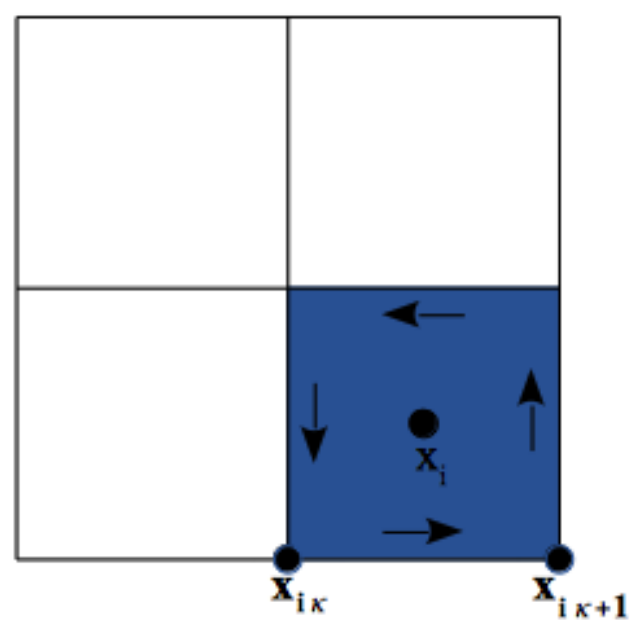

(a)



(b)

Figure 4.8: Line integral tracks for control volume methods: (a) cell-centered; (b) vertexcentered.

$$
\begin{aligned}
\int_{C_{i}}\left(\nu^{2} \nabla u \cdot \hat{\mathbf{n}}\right) d s & =\sum_{\kappa=1}^{N f_{i}} \int_{C_{i \kappa}}\left(\nu^{2} \nabla u \cdot \hat{\mathbf{n}}\right) d s \\
& =\sum_{\kappa=1}^{N f_{i}} \int_{\mathbf{x}_{\mathbf{i} \kappa}}^{\mathbf{x}_{\mathbf{i} \kappa+1}}\left(\nu^{2} \nabla u \cdot \hat{\mathbf{n}}\right) d s .
\end{aligned}
$$

\subsubsection{Midpoint rule}

The midpoint rule employed in computing equations 4.3 and 4.4 are given by

$$
\begin{gathered}
\sum_{\kappa=1}^{N f_{i}} \int_{\mathbf{x}_{\mathbf{i} \kappa}}^{\mathbf{x}_{\mathbf{i} \kappa+1}}(\eta \nabla v \cdot \hat{\mathbf{n}}) d s \approx\left\|\mathbf{x}_{\mathbf{i} \kappa+\mathbf{1}}-\mathbf{x}_{\mathbf{i} \kappa}\right\|[\eta \nabla v \cdot \hat{\mathbf{n}}]_{\mathbf{m}_{\mathbf{i} \kappa}}, \\
\sum_{\kappa=1}^{N f_{i}} \int_{\mathbf{x}_{\mathbf{i} \kappa}}^{\mathbf{x}_{\mathbf{i} \kappa+1}}\left(\nu^{2} \nabla u \cdot \hat{\mathbf{n}}\right) d s \approx\left\|\mathbf{x}_{\mathbf{i} \kappa+\mathbf{1}}-\mathbf{x}_{\mathbf{i} \kappa}\right\|\left[\nu^{2} \nabla u \cdot \hat{\mathbf{n}}\right]_{\mathbf{m}_{\mathbf{i} \kappa}},
\end{gathered}
$$

which results in the approximation

$$
\int_{C_{i}}(\eta \nabla v \cdot \hat{\mathbf{n}}) d s \approx \sum_{\kappa=1}^{N f_{i}}\left\|\mathbf{x}_{\mathbf{i} \kappa+\mathbf{1}}-\mathbf{x}_{\mathbf{i} \kappa}\right\|[\eta \nabla v \cdot \hat{\mathbf{n}}]_{\mathbf{m}_{\mathbf{i} \kappa}}
$$




$$
\int_{C_{i}}\left(\nu^{2} \nabla u \cdot \hat{\mathbf{n}}\right) d s \approx \sum_{\kappa=1}^{N f_{i}}\left\|\mathbf{x}_{\mathbf{i} \kappa+\mathbf{1}}-\mathbf{x}_{\mathbf{i} \kappa}\right\|\left[\nu^{2} \nabla u \cdot \hat{\mathbf{n}}\right]_{\mathbf{m}_{\mathbf{i} \kappa}},
$$

where

$$
\mathbf{m}_{\mathbf{i} \kappa}=\frac{1}{2}\left(\mathbf{x}_{\mathbf{i} \kappa}+\mathbf{x}_{\mathbf{i} \kappa+1}\right)
$$

represents the midpoint of the face.

Apropos the diffusive flux and source term components, they may be approximated as cell averages over the control volume, which involves estimating the volume $\Delta V_{i}$. This is achieved by evaluating these components at the cell centers and multiplying them by the volume of the cells. Thus, adding up the volume of every $V_{i}$ 's sub-control volumes. Since each sub-control volume (SCV) is a quadrilateral, then their volumes can be evaluated using the given formula

$$
S C V=\frac{1}{2}\left\|\left(\mathbf{b}_{\mathbf{1}}+\mathbf{a}_{\mathbf{1}}\right) \times\left(\mathbf{a}_{\mathbf{2}}-\mathbf{a}_{\mathbf{1}}\right)\right\|,
$$

with the cross product norm in two dimension in equation 4.10 given by

$$
\|\mathbf{p} \times \mathbf{q}\|=\left\|\left(p_{x}, p_{y}\right)^{\top} \times\left(q_{x}, q_{y}\right)^{\top}\right\|=\left|p_{x} q_{y}-p_{y} q_{x}\right|,
$$

where $\mathbf{p}$ and $\mathbf{q}$ are both arbitrary two-dimensional vectors.

\subsubsection{Gaussian quadrature}

In Section 3.2.2, Gaussian quadrature was reviewed as a method of evaluating more accurate approximations to the diffusive flux, viscous flux, velocity flux and source term components of 3.12

To begin with, the viscous and velocity flux components are considered respectively. In this instance, each integral for the viscous and velocity flux components

$$
\int_{C_{i \kappa}}(\eta \nabla v) \cdot \hat{\mathbf{n}} d s=\int_{\mathbf{x}_{\mathbf{i} \kappa}}^{\mathbf{x}_{\mathbf{i} \kappa+1}}(\eta \nabla v) \cdot \hat{\mathbf{n}} d s
$$


and

$$
\int_{C_{i \kappa}}\left(\nu^{2} \nabla u\right) \cdot \hat{\mathbf{n}} d s=\int_{\mathbf{x}_{\mathbf{i} \kappa}}^{\mathbf{x}_{\mathbf{i} \kappa+1}}\left(\nu^{2} \nabla u\right) \cdot \hat{\mathbf{n}} d s,
$$

respectively, should be evaluated with the Gaussian quadrature formula (equation 3.27). In order to do that, the following configuration for $C_{i \kappa}$ is introduced and given as

$$
\mathbf{x}(\gamma)=\frac{1}{2}(1-\gamma) \mathbf{x}_{\mathbf{i} \kappa}+\frac{1}{2}(1+\gamma) \mathbf{x}_{\mathbf{i} \kappa+\mathbf{1}}, \quad-1 \leq \gamma \leq 1
$$

such that

$$
\|\dot{\mathbf{x}}(\gamma)\|=\frac{1}{2}\left\|\mathbf{x}_{\mathbf{i} \kappa+\mathbf{1}}-\mathbf{x}_{\mathbf{i} \kappa}\right\|
$$

Applying equations 4.14 and 4.15 to equations 4.12 and 4.13 yields

$$
\begin{aligned}
\int_{C_{i \kappa}}(\eta \nabla v \cdot \hat{\mathbf{n}}) d s & =\int_{-1}^{1}(\eta \nabla v \cdot \hat{\mathbf{n}})\|\dot{\mathbf{x}}(\gamma)\| d \gamma \\
& =\frac{1}{2}\left\|\mathbf{x}_{\mathbf{i} \kappa+\mathbf{1}}-\mathbf{x}_{\mathbf{i} \kappa}\right\| \int_{-1}^{1}(\eta \nabla v \cdot \hat{\mathbf{n}}) d \gamma
\end{aligned}
$$

and

$$
\begin{aligned}
\int_{C_{i \kappa}}\left(\nu^{2} \nabla u \cdot \hat{\mathbf{n}}\right) d s & =\int_{-1}^{1}\left(\nu^{2} \nabla u \cdot \hat{\mathbf{n}}\right)\|\dot{\mathbf{x}}(\gamma)\| d \gamma \\
& =\frac{1}{2}\left\|\mathbf{x}_{\mathbf{i} \kappa+\mathbf{1}}-\mathbf{x}_{\mathbf{i} \kappa}\right\| \int_{-1}^{1}\left(\nu^{2} \nabla u \cdot \hat{\mathbf{n}}\right) d \gamma
\end{aligned}
$$

respectively.

The controversy surrounding the number of Gaussian quadrature points to use is dealt with by a decision to either apply the cell-centered or vertex-centered method from Section 4.2 to form the control volumes. Generally, a four-point or nine-point Gaussian quadrature can be applied to compute each integral in a control volume (see figure 4.9). The abscissas and weights up to a five-point Gaussian quadrature are provided in (Davis \& Rabinowitz, 1956; Love, 1966), but higher points for Gaussian quadrature are available in various literature. For a four-point Gaussian quadrature (figure 4.9a), the abscissas and weights 


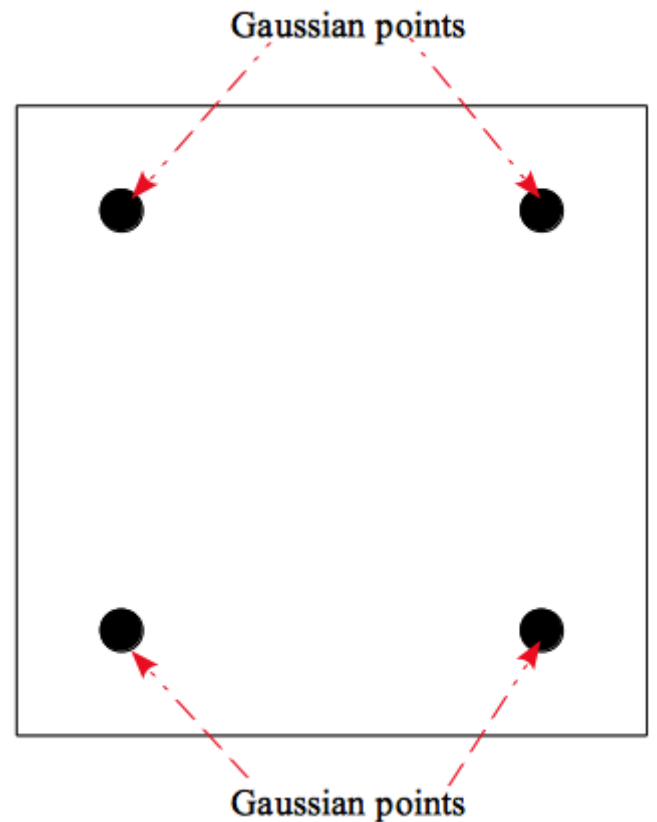

(a)

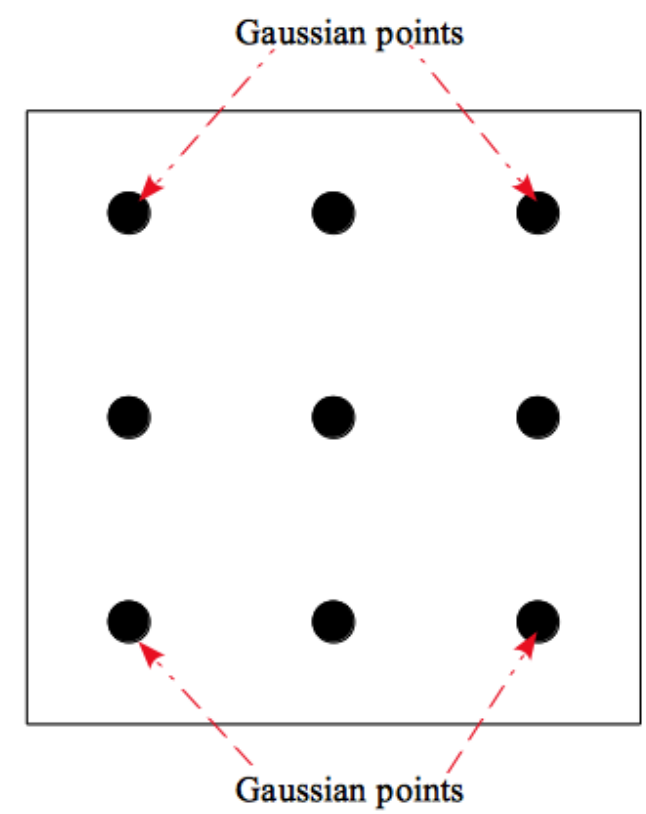

(b)

Figure 4.9: Gaussian quadrature points in a control volume: (a) four-point Gaussian quadrature; (b) nine-point Gaussian quadrature.

are such that

$$
\begin{aligned}
\int_{-1}^{1} f(\gamma) \mathrm{d} \gamma \approx & \frac{1}{36}(18+\sqrt{30}) f\left(-\frac{1}{35} \sqrt{525-70 \sqrt{30}}\right) \\
+ & \frac{1}{36}(18-\sqrt{30}) f\left(-\frac{1}{35} \sqrt{525+70 \sqrt{30}}\right) \\
& +\frac{1}{36}(18+\sqrt{30}) f\left(\frac{1}{35} \sqrt{525-70 \sqrt{30}}\right) \\
& +\frac{1}{36}(18-\sqrt{30}) f\left(\frac{1}{35} \sqrt{525+70 \sqrt{30}}\right)
\end{aligned}
$$

Applying equation 4.18 to equations 4.16 and 4.17 yields

$$
\begin{aligned}
\int_{C_{i \kappa}}(\eta \nabla v \cdot \hat{\mathbf{n}}) d s & \approx \frac{1}{2}\left\|\mathbf{x}_{\mathbf{i} \kappa+\mathbf{1}}-\mathbf{x}_{\mathbf{i} \kappa}\right\| \\
& \times\left\{[\eta \nabla v \cdot \hat{\mathbf{n}}]_{\mathbf{x}(\gamma 1)}+\ldots+[\eta \nabla v \cdot \hat{\mathbf{n}}]_{\mathbf{x}(\gamma 4)}\right\}
\end{aligned}
$$


and

$$
\begin{aligned}
\int_{C_{i \kappa}}\left(\nu^{2} \nabla u \cdot \hat{\mathbf{n}}\right) d s & \approx \frac{1}{2}\left\|\mathbf{x}_{\mathbf{i} \kappa+\mathbf{1}}-\mathbf{x}_{\mathbf{i} \kappa}\right\| \\
& \times\left\{\left[\nu^{2} \nabla u \cdot \hat{\mathbf{n}}\right]_{\mathbf{x}(\gamma 1)}+\ldots+\left[\nu^{2} \nabla u \cdot \hat{\mathbf{n}}\right]_{\mathbf{x}(\gamma 4)}\right\}
\end{aligned}
$$

respectively, where

$$
\begin{aligned}
\mathbf{x}(\gamma 1) & =\mathbf{x}\left(-\frac{1}{35} \sqrt{525-70 \sqrt{30}}\right) \\
& =\frac{1}{2}\left(1+\left(\frac{1}{35} \sqrt{525-70 \sqrt{30}}\right)\right) \mathbf{x}_{\mathbf{i}}+\frac{1}{2}\left(1-\left(\frac{1}{35} \sqrt{525-70 \sqrt{30}}\right)\right) \mathbf{x}_{\mathbf{i} \kappa+\mathbf{1}} \\
& =\frac{1}{2}\left[\left(\mathbf{x}_{\mathbf{i}}+\mathbf{x}_{\mathbf{i} \kappa+\mathbf{1}}\right)-\left(\frac{1}{35} \sqrt{525-70 \sqrt{30}}\right)\left(\mathbf{x}_{\mathbf{i} \kappa+\mathbf{1}}-\mathbf{x}_{\mathbf{i}}\right)\right]
\end{aligned}
$$

$$
\begin{aligned}
\mathbf{x}(\gamma 2) & =\mathbf{x}\left(-\frac{1}{35} \sqrt{525+70 \sqrt{30}}\right) \\
& =\frac{1}{2}\left(1+\left(\frac{1}{35} \sqrt{525+70 \sqrt{30}}\right)\right) \mathbf{x}_{\mathbf{i}}+\frac{1}{2}\left(1-\left(\frac{1}{35} \sqrt{525+70 \sqrt{30}}\right)\right) \mathbf{x}_{\mathbf{i} \kappa+\mathbf{1}} \\
& =\frac{1}{2}\left[\left(\mathbf{x}_{\mathbf{i}}+\mathbf{x}_{\mathbf{i} \kappa+\mathbf{1}}\right)-\left(\frac{1}{35} \sqrt{525+70 \sqrt{30}}\right)\left(\mathbf{x}_{\mathbf{i} \kappa+\mathbf{1}}-\mathbf{x}_{\mathbf{i}}\right)\right]
\end{aligned}
$$

$$
\begin{aligned}
\mathbf{x}(\gamma 3) & =\mathbf{x}\left(\frac{1}{35} \sqrt{525-70 \sqrt{30}}\right) \\
& =\frac{1}{2}\left(1-\left(\frac{1}{35} \sqrt{525-70 \sqrt{30}}\right)\right) \mathbf{x}_{\mathbf{i}}+\frac{1}{2}\left(1+\left(\frac{1}{35} \sqrt{525-70 \sqrt{30}}\right)\right) \mathbf{x}_{\mathbf{i} \kappa+\mathbf{1}} \\
& =\frac{1}{2}\left[\left(\mathbf{x}_{\mathbf{i}}+\mathbf{x}_{\mathbf{i} \kappa+\mathbf{1}}\right)+\left(\frac{1}{35} \sqrt{525-70 \sqrt{30}}\right)\left(\mathbf{x}_{\mathbf{i} \kappa+\mathbf{1}}-\mathbf{x}_{\mathbf{i}}\right)\right],
\end{aligned}
$$


and likewise

$$
\begin{aligned}
\mathbf{x}(\gamma 4) & =\mathbf{x}\left(\frac{1}{35} \sqrt{525-70 \sqrt{30}}\right) \\
& =\frac{1}{2}\left(1-\left(\frac{1}{35} \sqrt{525-70 \sqrt{30}}\right)\right) \mathbf{x}_{\mathbf{i}}+\frac{1}{2}\left(1+\left(\frac{1}{35} \sqrt{525-70 \sqrt{30}}\right)\right) \mathbf{x}_{\mathbf{i} \kappa+\mathbf{1}} \\
& =\frac{1}{2}\left[\left(\mathbf{x}_{\mathbf{i}}+\mathbf{x}_{\mathbf{i} \kappa+\mathbf{1}}\right)+\left(\frac{1}{35} \sqrt{525-70 \sqrt{30}}\right)\left(\mathbf{x}_{\mathbf{i} \kappa+\mathbf{1}}-\mathbf{x}_{\mathbf{i}}\right)\right]
\end{aligned}
$$

In the case of a nine-point Gaussian quadrature, Figure 4.9b illustrates how line integrals are needed to estimate the flux between adjacent control volumes. Nine-point Gaussian quadrature is used to compute these integrals. The abscissas and weights for the nine-point Gaussian quadrature provided in Davis and Rabinowitz (1956); Love (1966) is computed with the expression

$$
\begin{aligned}
\int_{-1}^{1} f(\gamma) \mathrm{d} \gamma & \approx \frac{25}{81}(\gamma 1+\gamma 3+\gamma 7+\gamma 9) \\
& +\frac{40}{81}(\gamma 2+\gamma 4+\gamma 6+\gamma 8)+\frac{64}{81} \gamma 5
\end{aligned}
$$

It is worthy of mentioning that the notation for the Gaussian abscissas used in Davis and Rabinowitz (1956); Love (1966) are used interchangeable with $\gamma$, but in effect, they mean the same thing. Refer to Davis and Rabinowitz (1956); Love (1966) for the values of the Gaussian abscissas for $\gamma 9$ and higher. Applying equation 4.25 to equations 4.16 and 4.17 yields

$$
\begin{aligned}
\int_{C_{i \kappa}}(\eta \nabla v \cdot \hat{\mathbf{n}}) d s & \approx \frac{1}{2}\left\|\mathbf{x}_{\mathbf{i} \kappa+\mathbf{1}}-\mathbf{x}_{\mathbf{i} \kappa}\right\| \\
& \times\left\{[\eta \nabla v \cdot \hat{\mathbf{n}}]_{\mathbf{x}(\gamma 1)}+\ldots+[\eta \nabla v \cdot \hat{\mathbf{n}}]_{\mathbf{x}(\gamma 9)}\right\}
\end{aligned}
$$

and

$$
\begin{aligned}
& \int_{C_{i \kappa}}\left(\nu^{2} \nabla u \cdot \hat{\mathbf{n}}\right) d s \approx \frac{1}{2}\left\|\mathbf{x}_{\mathbf{i} \kappa+\mathbf{1}}-\mathbf{x}_{\mathbf{i} \kappa}\right\| \\
& \times\left\{\left[\nu^{2} \nabla u \cdot \hat{\mathbf{n}}\right]_{\mathbf{x}(\gamma 1)}+\ldots+\left[\nu^{2} \nabla u \cdot \hat{\mathbf{n}}\right]_{\mathbf{x}(\gamma 9)}\right\}
\end{aligned}
$$


respectively, where $\mathbf{x}(\gamma 1), \mathbf{x}(\gamma 2), \ldots, \mathbf{x}(\gamma 9)$ are computed in the same processes as was done with the four-point Gaussian quadrature.

It is compelling to compare the accuracy of the Gaussian quadrature integration schemes with that of the midpoint integration scheme for the control volumes. Figure 4.10 compares the accuracy provided by the Gaussian quadrature scheme, including the midpoint rule, for meshes of various refinements. The result was produced with the analytic solution given by

$$
f(x)=\sin (x) \times \cos (x), \quad 20 \leq n \leq 0 .
$$

where $n$ represents the mesh intervals. The plot compares the integration methods on a vertical log scale and it is obvious that the errors of the midpoint rule, which is sometimes referred to as the one-point Gaussian quadrature, decreases really slowly due to its lower order of accuracy. In comparison with the four-point and nine-point Gaussian quadrature, their errors decrease rapidly, approaching zero. The nine-point Gaussian quadrature is the fastest to approach zero. This demonstrates that the higher-order nine-point Gaussian is the most accurate line integral method (see figure 4.10). In this thesis, the nine-point Gaussian quadrature is implemented, mainly because it is more accurate. The oscillations in the error when using the six-point and nine-point Gaussian quadrature is because we approach machine precision.

The diffusive flux and source term components of equation 3.12 may be evaluated with the two dimensional Gaussian quadrature. Equation 3.27 can be extended to two dimensions as provided in Burden and Faires (2001) as

$$
\int_{-1}^{1} \int_{-1}^{1} g(x, \gamma) d \alpha d \beta \approx \sum_{j=1}^{n} \sum_{k=1}^{n} w_{j} w_{k} g\left(x_{j}, \gamma_{k}\right)
$$

with the weights, $w_{i}$ and abscissas, $x_{i}$ still provided in Davis and Rabinowitz (1956); Love (1966). A mapping of each sub-control volume to $[-1,1] \times[-1,1]$ is required in order to apply equation 4.29 to the diffusive flux and source term components. The following configuration which involves the sub-control vertices, say $\mathbf{G}_{\mathbf{i}}$, can be used to achieve this 


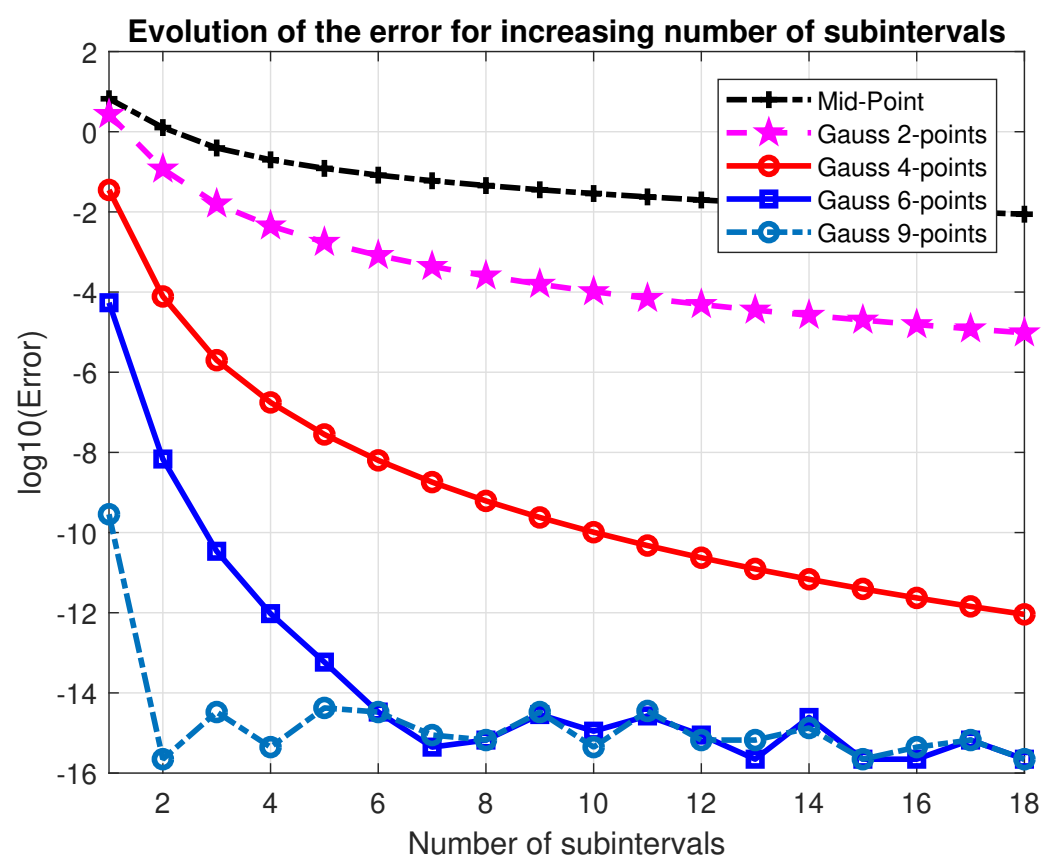

Figure 4.10: Accuracy of line integral methods.

(refer to Figure 4.5b)

$$
\begin{aligned}
\mathbf{x}(\alpha, \beta) & =\frac{1}{4}(1-\alpha)(1-\beta) \mathbf{G}_{\mathbf{1}}+\frac{1}{4}(1+\alpha)(1-\beta) \mathbf{G}_{\mathbf{2}} \\
& +\frac{1}{4}(1+\alpha)(1+\beta) \mathbf{G}_{\mathbf{3}}+\frac{1}{4}(1-\alpha)(1+\beta) \mathbf{G}_{\mathbf{4}} \\
& -1 \leq \alpha \leq 1, \quad-1 \leq \beta \leq 1 .
\end{aligned}
$$

The Jacobian of the transformation is also given as

$$
\left|\frac{\partial \mathbf{x}}{\partial(\alpha, \beta)}\right|=\left\|\frac{\partial \mathbf{x}}{\partial \alpha} \times \frac{\partial \mathbf{x}}{\partial \beta}\right\| .
$$

Applying equations 4.30 and 4.31 to the diffusive flux and source term components yield

$$
\begin{aligned}
\iint_{V_{i}} \zeta \cdot v d A & =\int_{-1}^{1} \int_{-1}^{1} \zeta(v(\mathbf{x}(\alpha, \beta)))\left\|\frac{\partial \mathbf{x}}{\partial \alpha} \times \frac{\partial \mathbf{x}}{\partial \beta}\right\| d \alpha d \beta \\
& \approx \sum_{j=1}^{n} \sum_{k=1}^{n} w_{j} w_{k} \zeta\left(v\left(\mathbf{x}\left(x_{j}, \gamma_{k}\right)\right)\right)\left\|\frac{\partial \mathbf{x}}{\partial \alpha} \times \frac{\partial \mathbf{x}}{\partial \beta}\right\|_{\left(x_{j}, \gamma_{k}\right)},
\end{aligned}
$$


and

$$
\begin{aligned}
\iint_{V_{i}} S d A & =\int_{-1}^{1} \int_{-1}^{1} S(u, v(\mathbf{x}(\alpha, \beta)))\left\|\frac{\partial \mathbf{x}}{\partial \alpha} \times \frac{\partial \mathbf{x}}{\partial \beta}\right\| d \alpha d \beta \\
& \approx \sum_{j=1}^{n} \sum_{k=1}^{n} w_{j} w_{k} S\left(u, v\left(\mathbf{x}\left(x_{j}, \gamma_{k}\right)\right)\right)\left\|\frac{\partial \mathbf{x}}{\partial \alpha} \times \frac{\partial \mathbf{x}}{\partial \beta}\right\|_{\left(x_{j}, \gamma_{k}\right)},
\end{aligned}
$$

for the diffusive flux and source components, respectively. It is worthy of mentioning that, equation 4.33 is only valid if the source term component depends on space.

\subsection{Interpolation method}

\subsubsection{Piecewise constant reconstruction}

In two dimensions, the piecewise constant reconstruction scheme can be applied to compute the fluxes through the cell faces, and based on this function reconstruction model, the variable values, $u$ and $v$, at the cell faces are interpolated.

The diffusive flux and source term components of equation 3.12 can be evaluated as simply the cell averages within each cell. In particular, the piecewise constant reconstruction may be applied to compute these flux components. This produces

$$
\int_{j-\frac{1}{2}}^{j+\frac{1}{2}} \int_{i-\frac{1}{2}}^{i+\frac{1}{2}}[\zeta \cdot v] \mathrm{d} x \mathrm{~d} y \approx \zeta \cdot V_{i, j}
$$

and

$$
\int_{j-\frac{1}{2}}^{j+\frac{1}{2}} \int_{i-\frac{1}{2}}^{i+\frac{1}{2}}(s) \mathrm{d} x \mathrm{~d} y \approx S_{i, j},
$$

respectively, where $S_{i, j}$ and $V_{i, j}$ are the cell averages of $s$ and $v$ at the control volume, $i, j$, respectively.

\subsubsection{Piecewise linear reconstruction}

In two dimensions, the piecewise linear reconstruction scheme can be applied to compute the fluxes through the cell faces, and based on this function reconstruction model, the 
variable values, $u$ and $v$, and their gradients, $\nabla u$ and $\nabla v$ at the cell faces are interpolated. Figure 4.11 shows the evolution of the fluxes in time. The integration is carried out in both



Figure 4.11: Evolution of fluxes in time at control volume faces.

the $x-$ and $y-$ directions over each cell volume. Figure 4.12 illustrates the piecewise linear reconstruction procedure for the variable gradients, $u$ and $v$, in one-dimension, however this idea can easily be transformed to suit the two-dimensional case. Here, $p$, $e$, and $w$, represent the cell faces, $i, i+\frac{1}{2}$, and $i-\frac{1}{2}$ respectively, while $P, E$, and $W$, represent the cell centres, $i, i+1$, and $i-1$, respectively.

The viscous flux and velocity flux components of equation 3.12 may be computed with the piecewise linear reconstruction in two-dimensions. This gives

$$
\begin{aligned}
& \int_{j-\frac{1}{2}}^{j+\frac{1}{2}} \int_{i-\frac{1}{2}}^{i+\frac{1}{2}}\left[\eta \nabla\left(\nabla v_{x}+\nabla v_{y}\right)\right] \mathrm{d} x \mathrm{~d} y \\
& \approx \eta\left[\left(\left(\frac{V_{i+1}-V_{i}}{\Delta x^{2}}\right)-\left(\frac{V_{i}-V_{i-1}}{\Delta x^{2}}\right)\right)+\left(\left(\frac{V_{j+1}-V_{j}}{\Delta y^{2}}\right)-\left(\frac{V_{j}-V_{j-1}}{\Delta y^{2}}\right)\right)\right],
\end{aligned}
$$




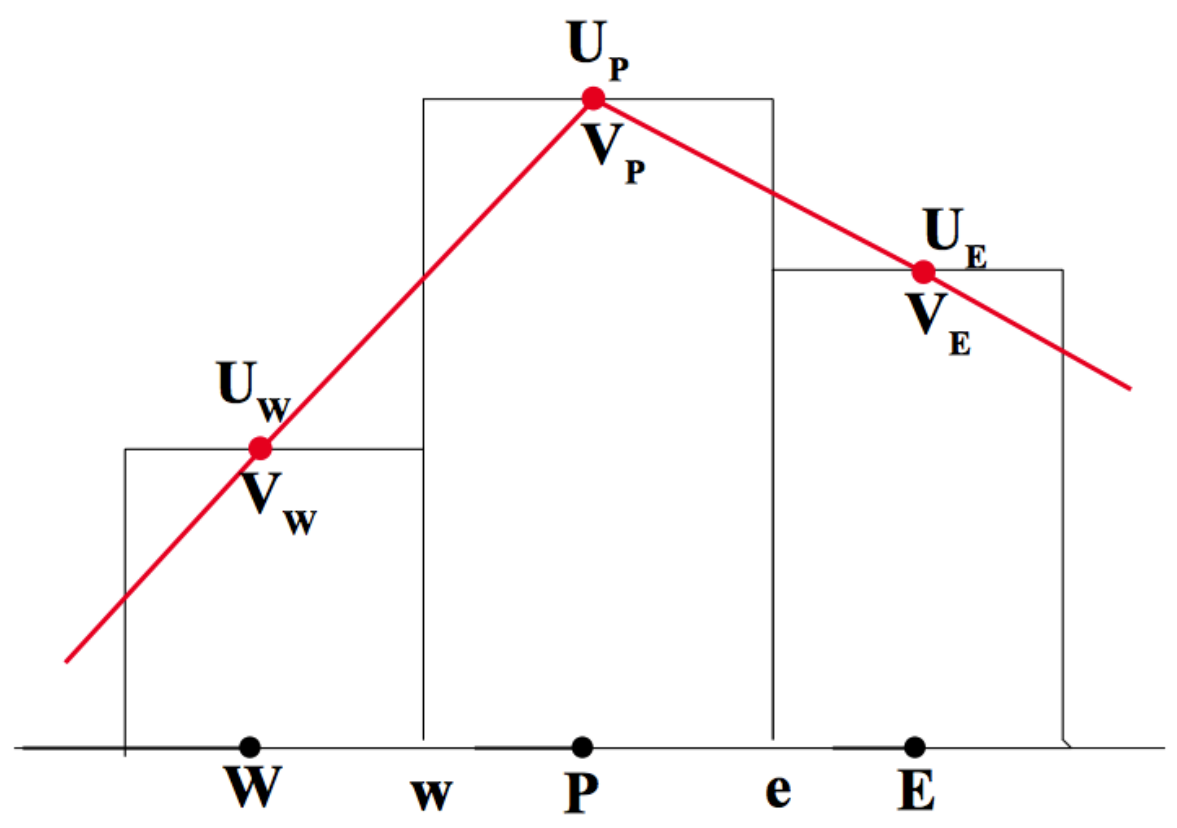

Figure 4.12: Piecewise Linear Reconstruction method applied to variable values, $u$ and $v$, adopted from Mensah et al. (2019).

and

$$
\begin{aligned}
& \int_{j-\frac{1}{2}}^{j+\frac{1}{2}} \int_{i-\frac{1}{2}}^{i+\frac{1}{2}}\left[\nu^{2} \nabla\left(\nabla u_{x}+\nabla u_{y}\right)\right] \mathrm{d} x \mathrm{~d} y \\
& \approx \nu^{2}\left[\left(\left(\frac{U_{i+1}-U_{i}}{\Delta x^{2}}\right)-\left(\frac{U_{i}-U_{i-1}}{\Delta x^{2}}\right)\right)+\left(\left(\frac{U_{j+1}-U_{j}}{\Delta y^{2}}\right)-\left(\frac{U_{j}-U_{j-1}}{\Delta y^{2}}\right)\right)\right],
\end{aligned}
$$

where $V_{i, j}$ and $U_{i, j}$ are cell averages of $v$ and $u$ at the control volume, $i, j$, respectively. The indexes $i$ and $j$ denote the $x-$ and $y-$ directions, accordingly.

\subsubsection{Shape functions}

The shape functions method in two dimensions, generates linear interpolants $u_{i}(x, y)$ and $v_{i}(x, y)$ over each control volume (quadrilateral element) (see Figure 4.6). If the control volume vertices are $\left(x_{1}, y_{1}\right),\left(x_{2}, y_{2}\right),\left(x_{3}, y_{3}\right)$ and $\left(x_{4}, y_{4}\right)$, then the shape function interpolant is described by

$$
u_{i}(x, y) \approx \sum_{i=1}^{4} N_{j}(x, y) u_{j}
$$


and

$$
v_{i}(x, y) \approx \sum_{i=1}^{4} N_{j}(x, y) v_{j},
$$

where the shape functions $N_{j}(x, y)$ are given by

$$
\begin{aligned}
& N_{1}=\frac{1}{4 x y}\left(x-x_{2}\right)\left(y-y_{4}\right), \\
& N_{2}=\frac{1}{4 x y}\left(x-x_{1}\right)\left(y-y_{3}\right), \\
& N_{3}=\frac{1}{4 x y}\left(x-x_{4}\right)\left(y-y_{2}\right),
\end{aligned}
$$

and similarly,

$$
N_{4}=\frac{1}{4 x y}\left(x-x_{3}\right)\left(y-y_{1}\right)
$$

With $u_{i}$ and $v_{i}$ given by equations 4.38 and $4.39, \nabla u_{i}$ and $\nabla v_{i}$ are thus, computed as

$$
\nabla u_{i}(x, y) \approx \sum_{i=1}^{4} \nabla N_{j}(x, y) u_{j}
$$

and

$$
\nabla v_{i}(x, y) \approx \sum_{i=1}^{4} \nabla N_{j}(x, y) v_{j},
$$

respectively, and are constant all through the control volume.

\subsection{Complete finite volume discretization}

The solution to the non-linear part of the finite volume discretization (equation 3.16) in two dimensions can now be achieved, with the schemes for integration and interpolation established (Ferguson, 1995; Ferguson \& Turner, 1996; Jayantha \& Turner, 2001, 2003; Turner et al., 2003). In the case of function reconstruction, $f_{i}$ is given as

$$
\begin{aligned}
f_{i}(\mathbf{u}, \mathbf{v}) & =-\zeta \cdot V_{i, j}+\eta\left[\left(\frac{V_{i+1}-2 V_{i}+V_{i-1}}{\Delta x^{2}}\right)+\left(\frac{V_{j+1}-2 V_{j}+V_{j-1}}{\Delta y^{2}}\right)\right] \\
& +\nu^{2}\left[\left(\frac{U_{i+1}-2 U_{i}+U_{i-1}}{\Delta x^{2}}\right)+\left(\frac{U_{j+1}-2 U_{j}-U_{j-1}}{\Delta y^{2}}\right)\right]+S_{i, j}
\end{aligned}
$$


In the case of the shape function method, $f_{i}$, is given by

$$
\begin{aligned}
f_{i}(\mathbf{u}, \mathbf{v}) & =\sum_{\kappa=1}^{N f_{i}}\left\|\mathbf{x}_{\mathbf{i} \kappa+\mathbf{1}}-\mathbf{x}_{\mathbf{i} \kappa}\right\|\left[-\zeta \cdot V\left(\mathbf{x}_{\mathbf{i}}\right) \Delta V_{i}+\eta\left(v_{i}\left(\mathbf{m}_{\mathbf{i} \kappa}\right)\right) \nabla v_{i}\left(\mathbf{m}_{\mathbf{i} \kappa}\right) \cdot \hat{\mathbf{n}}\right] \\
& +\left[\nu^{2}\left(u_{i}\left(\mathbf{m}_{\mathbf{i} \kappa}\right)\right) \nabla u_{i}\left(\mathbf{m}_{\mathbf{i} \kappa}\right) \cdot \hat{\mathbf{n}}\right]+S\left(\mathbf{x}_{\mathbf{i}}\right) \Delta V_{i},
\end{aligned}
$$

where $u_{i}$ and $v_{i}$ are the shape function interpolants (equation 4.47) and $\Delta V_{i}$ is the volume of the control volume, $V_{i}$, described by figure 4.8 .

\subsection{Summary}

In this Chapter, we have reviewed the two-dimensional considerations of the diffusiveviscous wave equation. We have extended the various spatial discretization techniques discussed in Chapter three to two-dimensions. Time integration schemes have not been discussed in this Chapter. In the next Chapter, we implement the two-dimensional considerations reviewed in this Chapter to a test case and we include a time integration technique. 


\section{Chapter 5}

\section{Diffusive-viscous wave modelling in two- dimensions with the simple Cartesian scheme}

In this Chapter, we investigate the effectiveness of the two-dimensional methods, in particular, the function reconstruction schemes and time integration scheme proposed in the previous Chapter by applying it to the two-dimensional diffusive-viscous wave equation. The first section of this chapter outlines the numerical model implemented and the meshing system applied. Subsequent sections deal with the numerical results and the order of accuracy of the numerical method.

\section{$5.1 \quad$ Numerical Model}

In order to conclude on the effectiveness of the function reconstruction schemes earlier reviewed, a test is carried out exclusively on the diffusive-viscous wave equation. The diffusive-viscous wave equation contains both space and temporal differential terms, and it is not possible to achieve a general analytical solution. Therefore, a numerical approach is imperative to approximate the solution. The transformed governing system of equations, equation 3.11 and 3.12, are expressed in vector components as provided below, that is, in velocity-displacement formulation (Mensah et al., 2019)

$$
\frac{\partial}{\partial t} \mathbf{Q}=\mathbf{L}(\mathbf{u}, \mathbf{v})+\mathbf{S}
$$


where the vector components,

$\mathbf{Q}=\left(\begin{array}{l}u \\ v\end{array}\right) ; \mathbf{L}=\left(\begin{array}{c}v \\ -\zeta \cdot v+\eta\left[\frac{\partial^{2} v}{\partial x^{2}}+\frac{\partial^{2} v}{\partial z^{2}}\right]+\nu^{2}\left[\frac{\partial^{2} u}{\partial x^{2}}+\frac{\partial^{2} u}{\partial z^{2}}\right]\end{array}\right) ; \mathbf{S}=\left(\begin{array}{l}0 \\ s\end{array}\right)$,

where we have implemented the source term, $s(t)=\left(1-2 \pi^{2} f^{2} t^{2}\right) \exp \left(-\pi^{2} f^{2} t^{2}\right)$, which is the so-called Ricker wavelet (where $f$ is referred to as the dominant frequency in Hertz and $t$ is the time in seconds).

\subsubsection{Meshing and function reconstruction}

The domain over which the system of equations are solved is divided up into a number of discrete volumes, as presented in figure 5.1, with each volume having a representative value situated at its center applying a finite volume scheme upon a Cartesian mesh in 2D. The finite volume scheme is the scheme employed to compute the spatial derivatives that appear in the governing equations. In previous chapters, the finite volume method was described as an integral method based on the integration of the equations over a finite volume. For a better insight, these integrals are later transformed applying the Gauss' divergence theorem where necessary. This physically implies that, fluxes flow through the control volume faces while flux balance over the control volume itself is satisfied. The conserved variable values are defined at each cell center and represents the average value over each cell. The fluxes are also computed at the interfaces between the cells. The second-order Runge-Kutta TVD time integration method is used to evolve the solution in time. Although certain methods such as the flux corrected transport finite difference method (Zhao, Gao, \& Zhao, 2014), reflectivity method (Zhao, Gao, Peng, \& Zhang, 2019) and the extended reflectivity method (Zhao, Gao, \& Peng, 2017) have been applied to the diffusive-viscous wave equation, the finite volume method can duly be appropriate for this problem, considering its straightforward physical interpretation, mass conservation and steep front approximations, especially in multi-dimensional problems (Manzini \& Ferraris, 2004). For a two-dimensional domain, the cells are enumerated with $(i, j)$ but for convenience, the compass notation, notable to many, which was invented by Imperial College (Patankar, 1980; Ferziger \& Peric, 2012) will be used when indicating 


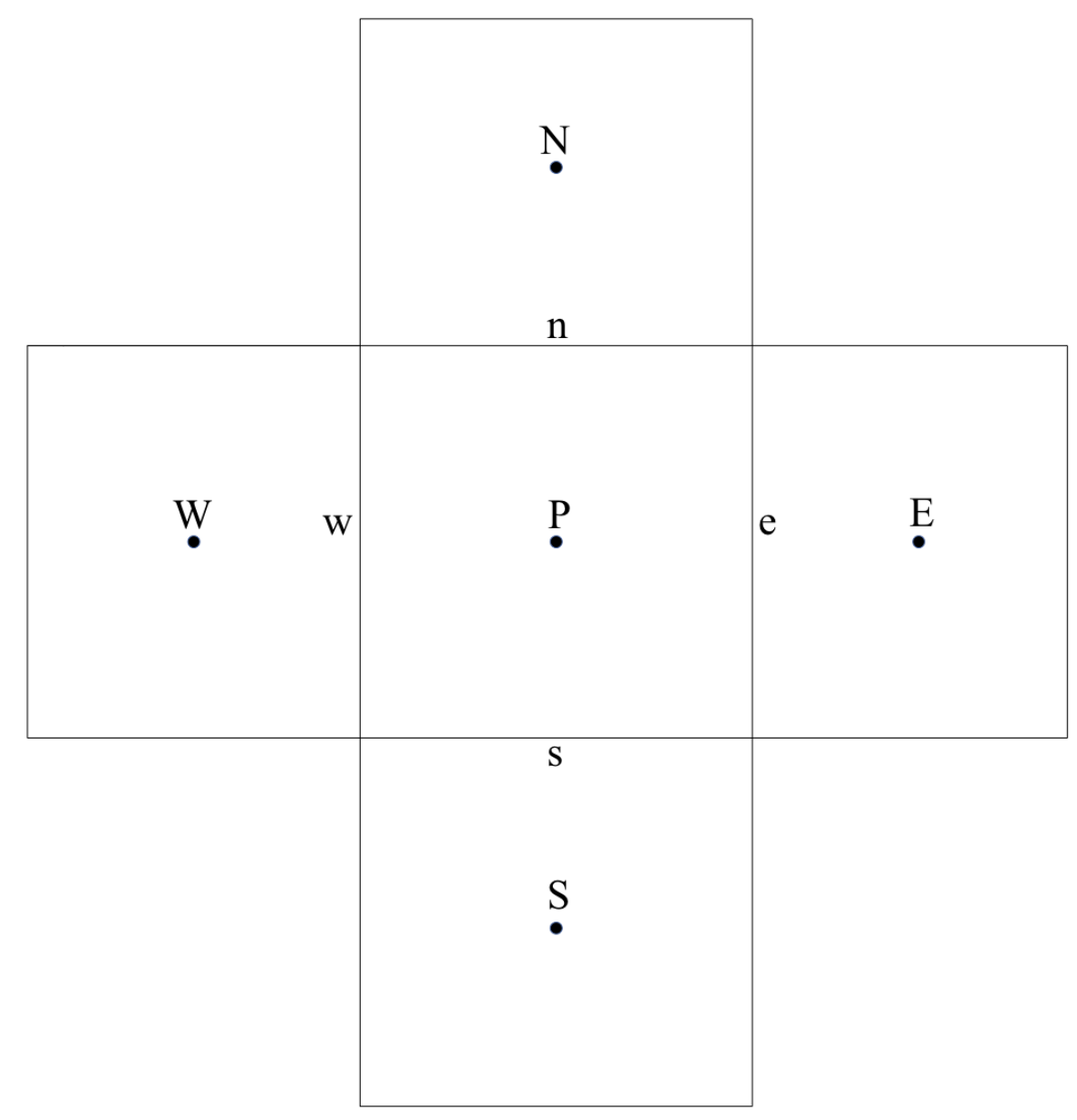

Figure 5.1: The Discretisation of a two-dimensional domain into Cartesian finite volumes adopted from Mensah et al. (2019).

the cell neighbours (refer to figure 5.1). The subscript $P$ denotes the cell upon which the equations are being discretized, with $E$ and $W$ (representing East and West) denoting its immediate neighbours in the $x$ direction and $N$ and $S$ (North and South) in the $y-$ direction. A capital letter denotes a value at the neighbouring cell center, that is the dependent variables, whilst a lower case denotes a value at the cell faces. Equation 5.1 is integrated term by term for the sake of clarity and readability.

We construct a semi-discrete finite volume scheme based on the function reconstruction method for the system of equations formulated in equation 5.1. To develop a finite volume discretization of equation 5.1, it is integrated using the piecewise linear reconstruction scheme, for all spatial components on a two-dimensional Cartesian mesh, in the $x-$ and $y$ - directions over the volume of the cell. Figure 4.12 describes the flux reconstruction 
procedure for the variable values and their gradients in one-dimension, however this principle can easily be transformed to suit the two-dimensional case and it is based on the above figures that the integration is developed. The semi-discrete form of equation 5.1 is given as

$$
\frac{d}{d t} \mathbf{Q}_{P}=\mathbf{L}_{P}(u(t), v(t))+\Gamma(t)
$$

where $\mathbf{Q}_{P}$ represents the cell average of the vector of unknowns $\mathbf{Q}$ in the control volume $P$ and $\mathbf{L}_{P}(u(t), v(t))$ represents the cell average of the operator appearing on the RHS of equation 5.1 over the control volume $P$. This operator is a vector with two components, say $\mathbf{L}_{P}=\left(\left(L_{P}\right)_{1},\left(L_{P}\right)_{2}\right)^{\top}$, the first of them given as the simple expression

$$
\left(L_{P}\right)_{1}=V_{P}(t)
$$

where $V_{P}(t)$ is the cell average of the function $v$ over the control volume $P$, which is computed using the piecewise constant reconstruction scheme. The second component of the operator is obtained as

$$
\begin{aligned}
\left(L_{P}\right)_{2} & =-\frac{\zeta}{\Delta x \Delta y} \int_{s}^{n} \int_{w}^{e} v d x d y+\frac{\eta}{\Delta x \Delta z} \int_{s}^{n} \int_{w}^{e}\left[\frac{\partial}{\partial x}\left(\frac{\partial v}{\partial x}\right)+\frac{\partial}{\partial y}\left(\frac{\partial v}{\partial y}\right)\right] d x d y \\
& +\frac{\nu^{2}}{\Delta x \Delta y} \int_{s}^{n} \int_{w}^{e}\left[\frac{\partial}{\partial x}\left(\frac{\partial u}{\partial x}\right)+\frac{\partial}{\partial y}\left(\frac{\partial u}{\partial y}\right)\right] d x d y .
\end{aligned}
$$

\subsubsection{Flux approximations and time integration}

The viscous flux and velocity flux components can be approximated with a piecewise linear reconstruction. This function approximation for the second order derivatives is equivalent to two nested central difference approximation. The diffusive flux component 
is evaluated as simply the cell average of the control volume. This is given as

$$
\begin{aligned}
\left(L_{P}\right)_{2} & =-\zeta \cdot V_{P}(t) \\
& +\eta\left[\left(\left(\frac{V_{E}(t)-V_{P}(t)}{\Delta x^{2}}\right)-\left(\frac{V_{P}(t)-V_{W}(t)}{\Delta x^{2}}\right)\right)\right] \\
& +\left[\left(\left(\frac{V_{N}(t)-V_{P}(t)}{\Delta y^{2}}\right)-\left(\frac{V_{P}(t)-V_{S}(t)}{\Delta y^{2}}\right)\right)\right] \\
& +\nu^{2}\left[\left(\left(\frac{U_{E}(t)-U_{P}(t)}{\Delta x^{2}}\right)-\left(\frac{U_{P}(t)-U_{W}(t)}{\Delta x^{2}}\right)\right)\right] \\
& +\left[\left(\left(\frac{U_{N}(t)-U_{P}(t)}{\Delta y^{2}}\right)-\left(\frac{U_{P}(t)-U_{S}(t)}{\Delta y^{2}}\right)\right)\right]
\end{aligned}
$$

The method of lines, usually applied in solving PDEs is employed. This involves initially discretizing only the spatial derivatives of equation 3.11 and 3.12 and leaving the time variable continuous, leading to a system of ordinary differential equations (ODEs) (equations 5.2, 5.3, and 5.4), for which an appropriate ODE solver must be employed. Owing to the second-order of accuracy of the spatial approximation developed using piecewise linear reconstruction, a second order solver should be employed for time integration. In this thesis, a second-order Runge-Kutta TVD scheme is applied (see Shu \& Osher, 1988; Gottlieb \& Shu, 1998, for details on Runge-Kutta TVD schemes), even though there are other possible alternatives, such as the MUSCL-Hancock scheme which has second-order of accuracy both in space and time. The second-order Runge-Kutta TVD scheme is expressed as (see Shu \& Osher, 1988);

$$
\begin{aligned}
Q_{P}^{(1)} & =Q_{P}^{n}+\Delta t \cdot \mathbf{L}_{P}\left(Q_{P}^{n}\right), \\
Q_{P}^{n+1} & =\frac{1}{2} Q_{P}^{n}+\frac{1}{2} Q_{P}^{(1)}+\frac{1}{2} \Delta t \cdot \mathbf{L}_{P}\left(Q_{P}^{(1)}\right) .
\end{aligned}
$$

The third component in equation 5.2 is the source term and it is evaluated as simply the cell average of the control volume as

$$
\Gamma(t)=\frac{1}{\Delta x \Delta y} \int_{s}^{n} \int_{w}^{e} s(t) d x d y=s(t)
$$

where $\Gamma(t)$ represents the cell average of the source term. Here, reference is made to 
equation 5.1.

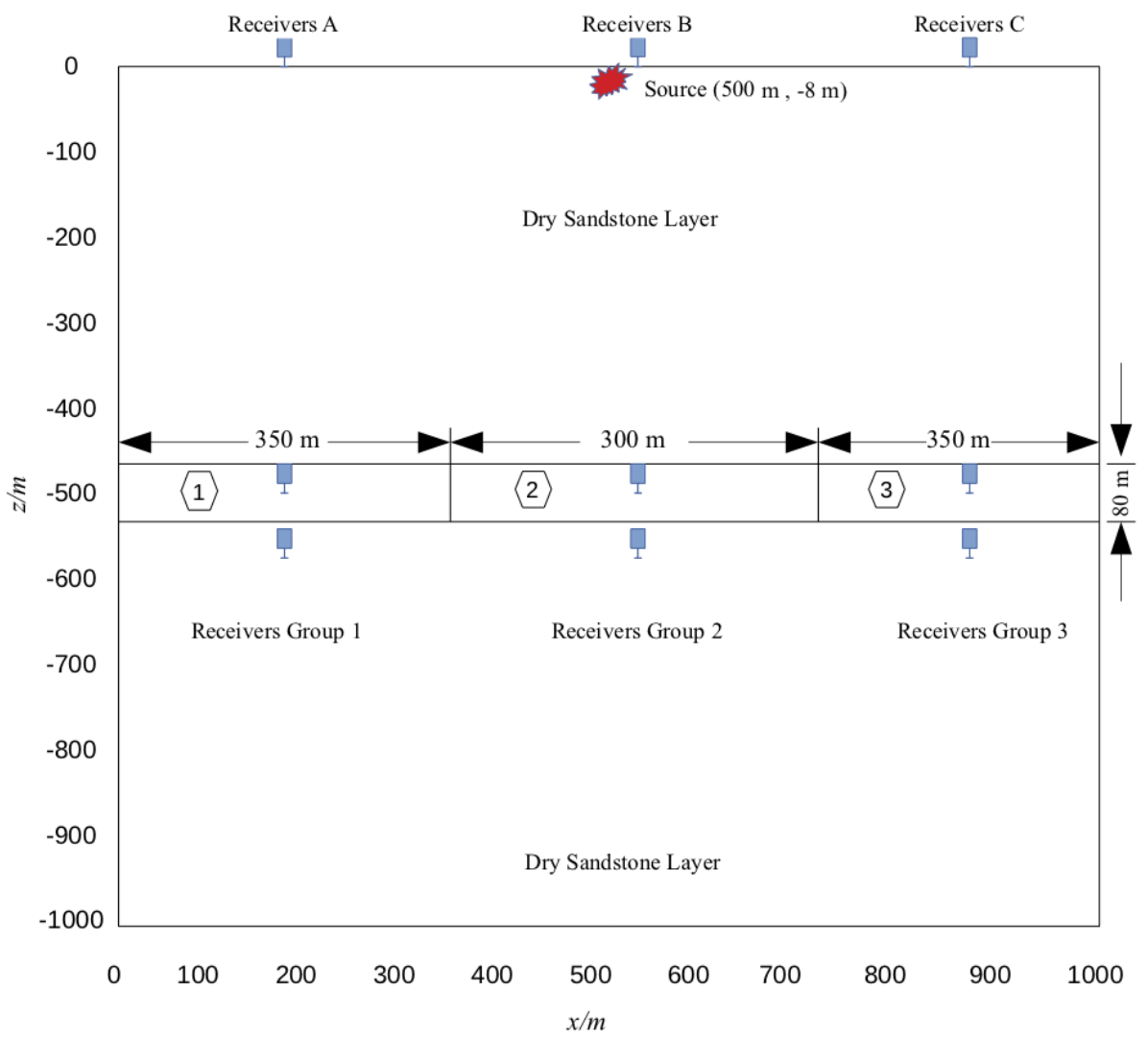

Figure 5.2: The fluid-saturated model with an $80 \mathrm{~m}$ thick (1)oil-, (2)water- and (3)gassaturated layer embedded in a homogeneous half space, adopted from Mensah et al. (2019).

\subsection{Error norm computation}

Let us consider a rectangular domain $[a, b] \times[c, d]$ discretized with a regular mesh of control volumes, each of them with area $\Delta x \times \Delta y$. We assume that the exact solution of our problem is $u(x, y, t)$ and we have obtained an approximated solution applying the finite volume method. Let us denote this approximate solution as $\tilde{u}(x, y, t)$, which is piecewise constant. We can define the $L_{1}$ norm of the error in the domain $[a, b] \times[c, d]$ as

$$
\|\epsilon\|_{1}=\int_{a}^{b} \int_{c}^{d}|u(x, y, t)-\tilde{u}(x, y, t)| d x d y \approx \Delta x \Delta y \sum_{k=1}^{n}\left|u_{k}-\tilde{u}_{k}\right|,
$$


where $u_{k}$ are the cell averages of the exact solution for each control volume, $\tilde{u}_{k}$ are the cell averages of the approximate solution for each control volume. We can also define the $L_{\infty}$ norm or maximum norm as

$$
\|\epsilon\|_{\infty}=\max _{1 \leq k \leq n}\left|u_{k}-\tilde{u}_{k}\right|
$$

There is also the $L_{2}$ norm which is defined as

$$
\|\epsilon\|_{2}=+\sqrt{\int_{a}^{b} \int_{c}^{d}|u(x, y, t)-\tilde{u}(x, y, t)|^{2} d x d y} \approx+\sqrt{\Delta x \Delta y \sum_{k=1}^{n}\left|u_{k}-\tilde{u}_{k}\right|^{2}}
$$

where $n$ is the number of control volumes.

If we wish to obtain the order of the method, we refine the mesh several times and obtain the error norm each time. Let us consider that we use $\Delta x_{1}=\Delta y_{1}=h_{1}$ and compute an error norm: $\left|\epsilon_{1}\right|$, then it is verified:

$$
\left|\epsilon_{1}\right| \leq C h_{1}^{p}
$$

where $\mathrm{C}$ is a constant and $\mathrm{p}$ is the sought order. Now we reduce the size of the discretization, that is $\Delta x_{2}=\Delta y_{2}=h_{2}$, usually we take $h_{2}=\frac{h_{1}}{2}$, then it is verified

$$
\left|\epsilon_{2}\right| \leq C h_{2}^{p}
$$

Now we can obtain the order $p$ as

$$
p=\frac{\log \left(\frac{\left|\epsilon_{1}\right|}{\left|\epsilon_{2}\right|}\right)}{\log \left(\frac{h_{1}}{h_{2}}\right)}
$$

This process is repeated several times until we observe the tendency of $p$ towards an integer value, which is the sought order of accuracy. 
Remark: if we take $h_{2}=\frac{h_{1}}{2}$ then (5.13) is replaced by

$$
p=\frac{\log \left(\frac{\left|\epsilon_{1}\right|}{\left|\epsilon_{2}\right|}\right)}{\log (2)}
$$

\subsection{Accuracy of the numerical scheme}

The following auxilliary problem, with know exact solution, is introduced for the purpose of testing the accuracy of the numerical scheme used in this work

$$
\left\{\begin{array}{c}
\frac{\partial u(x, z, t)}{\partial t}=v(x, z, t) \\
\frac{\partial v(x, z, t)}{\partial t}+\zeta v(x, z, t)-\eta \Delta v(x, z, t)-\nu^{2} \Delta u(x, z, t)=\Psi(x, z, t) \\
u(x, z, 0)=v(x, z, 0)=0 \\
\frac{\partial u(x,-\pi / 2, t)}{\partial z}=\frac{\partial v(x, \pi / 2, t)}{\partial z}=0 \\
\frac{\partial u(-\pi / 2, z, t)}{\partial x}=\frac{\partial u(\pi / 2, z, t)}{\partial x}=0
\end{array}\right.
$$

with $-\pi / 2 \leq(x, z) \leq \pi / 2, \quad \forall t>0$. The forcing term is $\Psi(x, z, t)=2 \lambda_{1}\left(\cos ^{2}(x)+\right.$ $\left.\cos ^{2}(z)-\sin (x) \sin (z)\right)+2(1+\zeta t) \sin (x) \sin (z)+4 \lambda_{2}$ where we have denoted $\lambda_{1}:=$ $-1-\zeta t-2 \nu^{2} t^{2}-4 \eta t$ and $\lambda_{2}:=1+\zeta t+\nu^{2} t^{2}+2 \eta t$.

The exact solution of equation 5.15 is provided as

$$
\left\{\begin{array}{l}
u(x, z, t)=t^{2}(\sin (x)+\sin (z))^{2} \\
v(x, z, t)=2 t(\sin (x)+\sin (z))^{2}
\end{array}\right.
$$

The numerical solution is obtained through the numerical scheme applied in this thesis, which is based on the finite volume method, with piecewise linear reconstruction of all flux components in space and second order Runge-Kutta TVD (Total Variation Diminishing) scheme in time. The values of the physical parameters considered are $\zeta=65.4 \mathrm{~Hz}$, $\eta=0.0147 \mathrm{~m}^{2} / \mathrm{s}$ and $\nu=10 \mathrm{~m} / \mathrm{s}$. It is worthy of noting that the values implemented for the diffusive attenuation parameter $(\zeta)$ and the viscosity attenuation parameter $(\nu)$ correspond to the oil saturated layer. The final output time applied is $t=0.25$ and the time 
Table 5.1: Convergence rates for problem (Eq. 5.15). Using $\zeta=65.4 \mathrm{~Hz}, \eta=$ $0.0147 \mathrm{~m}^{2} / \mathrm{s}$ and $\nu=10 \mathrm{~m} / \mathrm{s}$. Output time $t=0.25$. Adopted from Mensah et al. (2019).

\begin{tabular}{ccccc}
\hline Cells & $\|$ Error $\|_{2}$ & $\|$ Order $\|_{2}$ & $\|$ Error $\|_{2}$ & $\|$ Order $\|_{2}$ \\
\hline \multicolumn{3}{c}{ Velocity $(\mathrm{v})$} & \multicolumn{3}{c}{ Displacement $(\mathrm{u})$} \\
\hline $10 \times 10$ & $7.86 \times 10^{-3}$ & & $2.90 \times 10^{-3}$ \\
$20 \times 20$ & $3.11 \times 10^{-3}$ & 1.34 & $7.16 \times 10^{-4}$ & 2.02 \\
$40 \times 40$ & $8.35 \times 10^{-4}$ & 1.90 & $1.78 \times 10^{-4}$ & 2.01 \\
$60 \times 60$ & $3.78 \times 10^{-4}$ & 1.99 & $7.93 \times 10^{-5}$ & 2.00 \\
$80 \times 80$ & $2.13 \times 10^{-4}$ & 1.97 & $4.46 \times 10^{-5}$ & 2.00 \\
$120 \times 120$ & $9.52 \times 10^{-5}$ & 2.00 & $1.98 \times 10^{-5}$ & 2.01 \\
$160 \times 160$ & $5.36 \times 10^{-5}$ & 1.99 & $1.11 \times 10^{-5}$ & 2.01 \\
$200 \times 200$ & $3.43 \times 10^{-5}$ & 2.00 & $7.13 \times 10^{-6}$ & 1.99 \\
\hline
\end{tabular}

step is computed according to the formula given by

$$
\Delta t=\min \left(\alpha \Delta x^{2} / \eta, \alpha \Delta y^{2} / \eta, \alpha \Delta x^{2} / \nu, \alpha \Delta y^{2} / \nu\right)
$$

where the value $\alpha=0.75$ has been chosen on the account of stability reasons, even though no theoretical analysis on this value has been carried out.

The numerical convergence rates are illustated in Table 5.1 where it can be verified that the expected second order of accuracy is obtained in practice, for both variable values: velocity and displacement.

In figure 5.3, a comparison of the exact solution (equation 5.16) of the auxiliary problem (equation 5.15) with the numerical solutions obtained on three different meshes $(30 \times 30$, $60 \times 60$ and $120 \times 120)$ for the output time $t=0.25$ is illustrated. For the sake of comparison, the solutions are demonstrated for a constant value of $x$, that is, $x=0.5$. The top frame depicts the displacement (variable $u$ ), while the bottom frame shows the velocity (variable $v$ ).

\subsection{Numerical Results}

In this thesis, we investigate a $[0,1000] \times[0,1000] \mathrm{m}$ fluid-saturated model in twodimensions (see figure 5.2). A finite volume cartesian regular mesh of dimension, $500 \times$ 


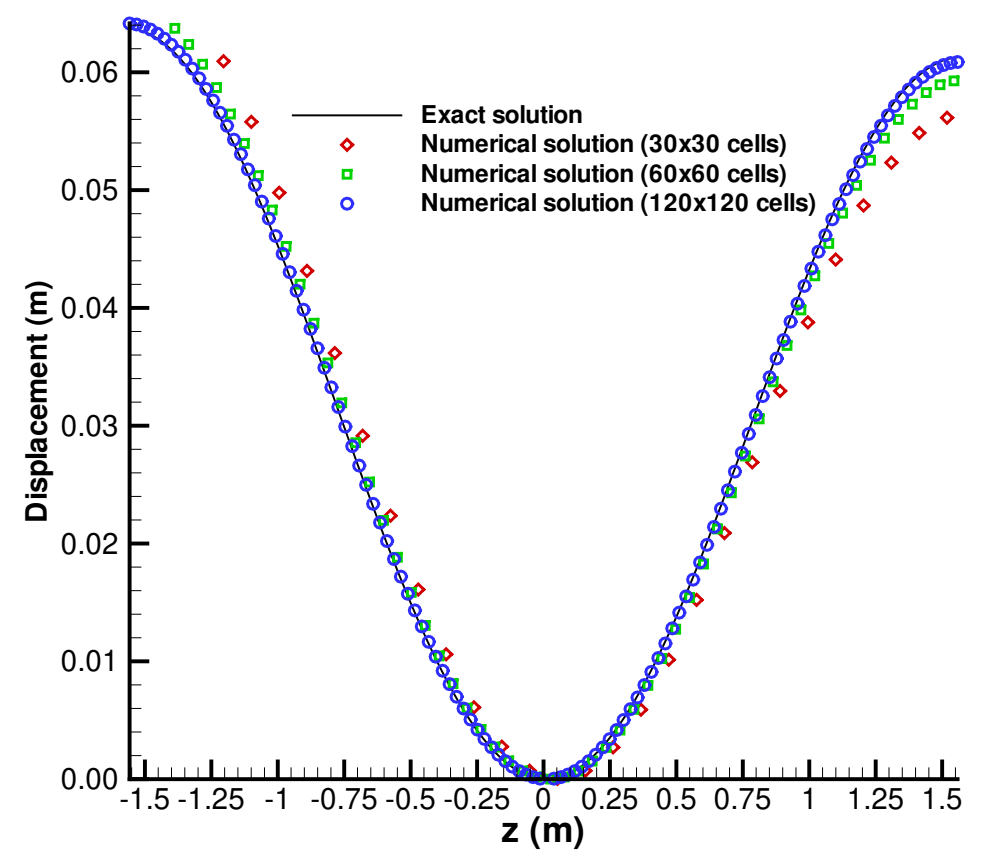

(a)

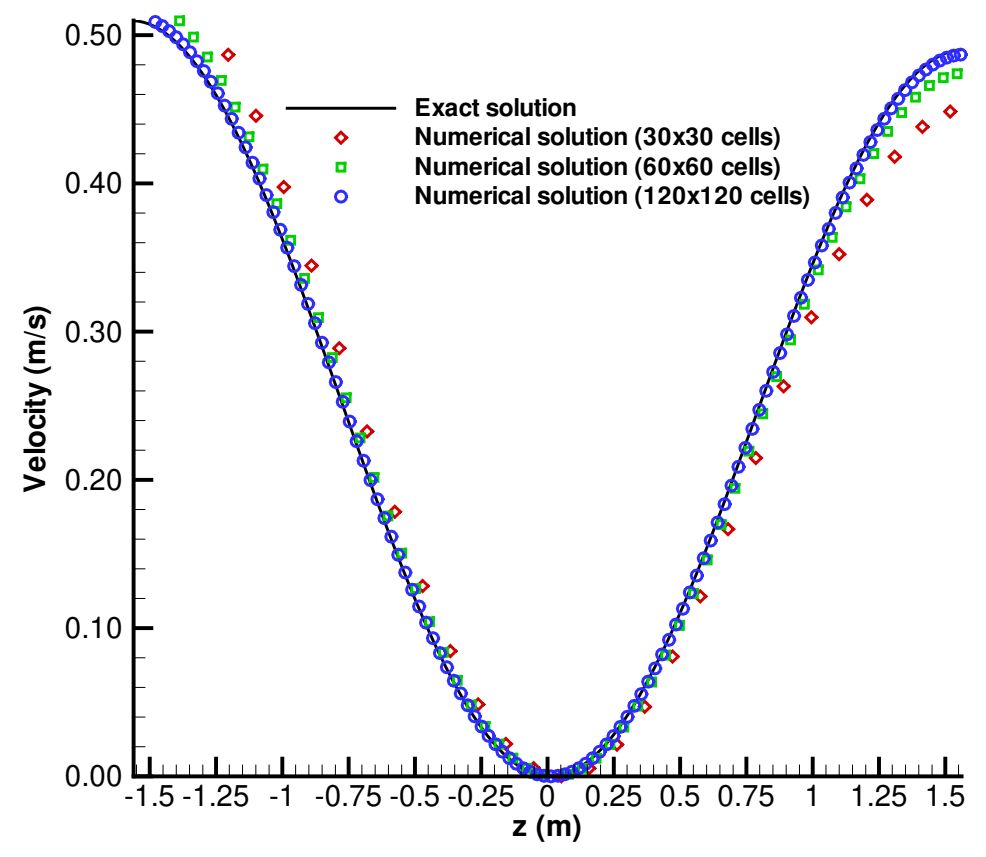

(b)

Figure 5.3: Comparison of the exact solution (5.16) of the problem (5.15) with the numerical solutions obtained on three different meshes for output time $t=0.25$ cutting the surface with the plane $x=0.5$. Top: Displacement. Bottom: Velocity. Adopted from Mensah et al. (2019). 
500 control volumes, with an $80 \mathrm{~m}$ thick oil-, water-, and gas-saturated layer embedded within two dry sandstone layers is generated. Situated at the center of the fluid saturated layer, as shown in figure 5.2 above, is the water-saturated layer which is $300 \mathrm{~m}$ in length with the two other layers (oil and gas) having lengths of $350 \mathrm{~m}$ each. The physical parameters applied to the model are the same as those used by Mensah et al. (2019) and are presented in Table 5.2.

Table 5.2: Physical Parameters of the Model, adopted from Mensah et al. (2019)

\begin{tabular}{|l|c|c|c|}
\hline Medium & $\nu(\mathrm{m} / \mathrm{s})$ & $\zeta(\mathrm{Hz})$ & $\eta\left(\mathrm{m}^{2} / \mathrm{s}\right)$ \\
\hline Dry Sandstone Layer & 1600.0 & 0.0 & 0.0 \\
Oil-Saturated Layer & 1015.0 & 65.4 & 0.0147 \\
Gas-Saturated Layer & 630.0 & 42.68 & 0.0112 \\
Water-Saturated Layer & 1470.0 & 90.0 & 0.02 \\
\hline
\end{tabular}

\subsubsection{Source and receiver implementation}

There are various seismic sources used in seismic numerical simulations as well as for both land and marine acquisition surveys. An important aspect of evaluating the efficiency of the seismic source is the measurement of its spectral characteristics, such as phase, frequency, amplitude, among others, and it is quite tedious to estimate these spectral characteristics accurately. The generation of an impulse or a Dirac spike is the overall attempt to produce a better seismogram.

The source implemented is the so-called Ricker wavelet with an amplitude spectrum peak of $30 \mathrm{~Hz}$. Figure 2.1 illustrate a Ricker wavelet with a peak amplitude spectrum of 30 $\mathrm{Hz}$, and its corresponding frequency spectrum. The source is integrated over the timestep and then distributed over the cells. Empirical studies have typically demonstrated that the Ricker wavelet better approximates the seismic spectra than other families of approximating functions and it, at the same time, requires fewer parameters than other possible approximations. It is for these reasons that the Ricker wavelet is implemented in this thesis. The source is positioned at the center of the surface (refer to figure 5.2). The source delay is $40 \mathrm{~ms}$ and the step-sizes are $2.0 \mathrm{~m}$ for both $x$ - and $z$-directions.

The time-step is controlled by the von Neumann Stability Criteria for the diffusive-viscous 


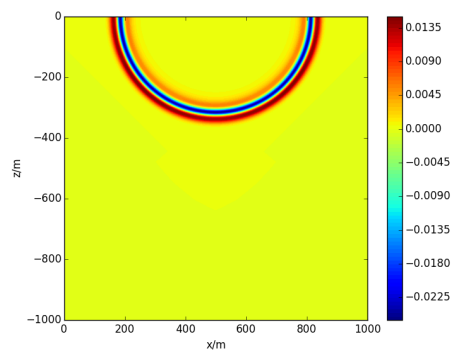

(a) $239 \mathrm{~ms}$



(d) $239 \mathrm{~ms}$

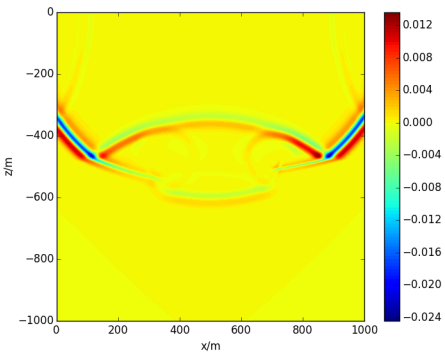

(b) $419 \mathrm{~ms}$

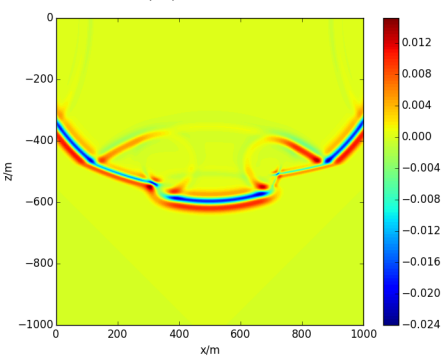

(e) $419 \mathrm{~ms}$

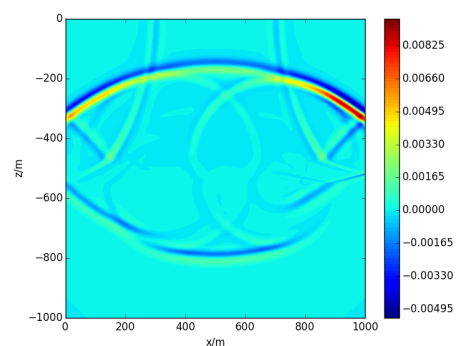

(c) $539 \mathrm{~ms}$

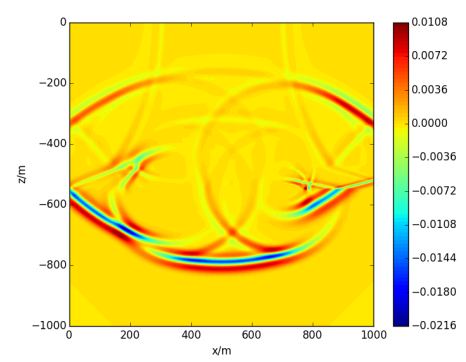

(f) $539 \mathrm{~ms}$

Figure 5.4: Snapshots of diffusive-viscous wavefields (a), (b), (c) and acoustic wavefields (d), (e), (f) in a lateral heterogeneous medium for Particle Displacement (notice varying color scaling), adopted from Mensah et al. (2019).

wave equation discretised with the finite difference method, as proposed by Zhao, Gao, and Zhao (2014), and which is given as

$$
0 \leq d t \leq \min \left(\frac{\sqrt{6}}{4} \frac{h}{\nu}, \frac{h^{2}}{\zeta h^{2}+8 \eta}\right)
$$

where "min" describes the minimum value of the quantities and $h$ depicts $\Delta x$ and $\Delta y$. The minimum value of the quantities in our case is $0.765 \mathrm{~ms}$ but in our computations, a larger time-step is implemented and the scheme is still stable. The time-step used in this case is $0.8 \mathrm{~ms}$. The total time for the simulation is $700 \mathrm{~ms}$. To better visualize the attenuation effects occurring within the fluid saturated layer, three receivers are placed at the surface with two other receivers placed below and parallel to each of the receivers at surface (refer to figure 5.2) but within the fluid saturated layer. Results are presented for both the particle motions (velocity and displacement) since a system of equation has been developed, i.e., velocity-displacement formulations.

Snapshots are presented for particle motions (displacement and velocity) in figures 5.4 


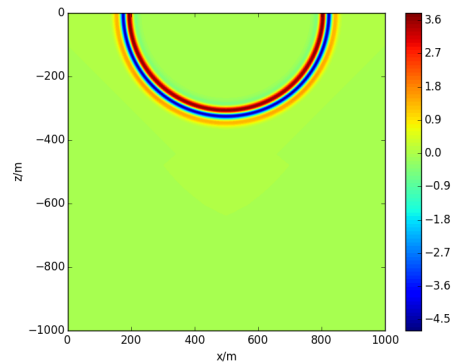

(a) $239 \mathrm{~ms}$



(d) $239 \mathrm{~ms}$

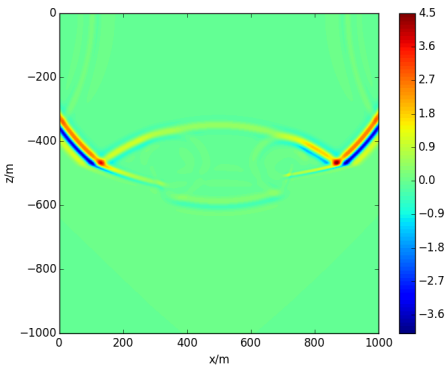

(b) $419 \mathrm{~ms}$



(e) $419 \mathrm{~ms}$

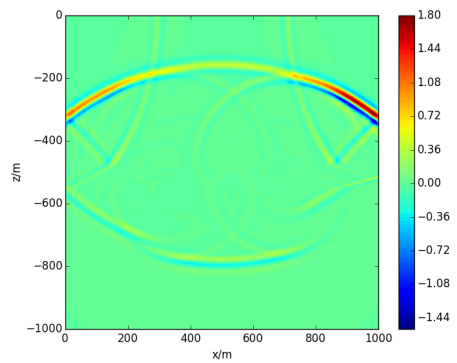

(c) $539 \mathrm{~ms}$



(f) $539 \mathrm{~ms}$

Figure 5.5: Snapshots of diffusive-viscous wavefields (a), (b), (c) and acoustic wavefields (d), (e), (f) in a lateral heterogeneous medium for Particle Velocity (notice varying color scaling), adopted from Mensah et al. (2019).

and 5.5 respectively at randomly chosen times. In the layout of the presentation of both cases, snapshots for the diffusive-viscous waves are presented on top, in each case, with snapshots of the acoustic waves arranged beneath snapshots of the former.

Seismograms resulting from receivers A, B, C, and receiver groups 1, 2 and 3 (refer to figure 5.2) are presented and comparisons are made between those generated for both the acoustic and diffusive-viscous waves. It is worthy of mentioning that the information from the oil-saturated region is recorded by receiver group 1, receiver group 2 also records for the water-saturated region, and receiver group 3 records for the gas-saturated region. Multiple reflections generated from the layer are as well indicated on the seismograms and it is realized that whilst the multiples from the water-, oil- and gas-saturated layers are clearly visible in the case of the acoustic waves, they are nearly lurking in the case of the diffusive and viscous waves(figures 5.6, 5.7 and 5.8). In comparison with seismograms from the acoustic case, seismograms from the diffusive-viscous case suggests an obvious amplitude attenuation which appears sharply within the model but most essentially, within the fluid saturated layer. It worthy of note that seismic simulation of 
wavefields without "inter-bed" multiple reflections before imaging is often required in practice since the presence of these "inter-bed" multiple reflections have a strong impact on the interpretation which distorts the wavelet of true reflections and also reduces the quality of final images. The relative time positions, for particle velocity motions and particle displacement motions for both the acoustic and diffusive-viscous scenarios, of the amplitude attenuation of receiver $\mathrm{A}$ is about $0.24 \mathrm{~s}$, with receiver B occuring at about 0.05 $\mathrm{s}$, and ultimately receiver $\mathrm{C}$ resulting at about $0.25 \mathrm{~s}$ (refer to figure 5.6).

From the numerical results, the same snapshots are produced for both the acoustic and diffusive-viscous cases, $239 \mathrm{~ms}$ after the source has propagated from the source location within the first dry sandstone layer. It is obvious from figures 5.4 and 5.5 that there is a significant attenuation as the diffusive and viscous waves propagate through the fluidsaturated layer for both displacement and velocity results. It is worth noting that the results obtained for the oil- and water-saturated media are similar to those obtained by Mensah et al. (2019); Zhao, Gao, and Zhao (2014). The gas-saturated layer displays a lower attenuating effect when compared to both the oil- and water-saturated media. This low attenuating effect is apparent, not only on the snapshots, but on the seismograms as well. The low attenuating characteristics of the gas-saturated layer can be associated to the low values of the diffusive and viscous attenuation parameters, $\zeta$ and $\eta$ accordingly, as compared to diffusive and viscous attenuation parameters of the oil- and water-saturated layers. The layer with higher attenuating parameters, i.e. water-saturated layer, demonstrates a stronger attenuating effect. Thus, it can be concluded that the attenuation parameters significantly affect numerical results, and these parameters which can be obtained from laboratory analysis or through seismic inversion from seismic field data, depend primarily on permeability, stiffness, porosity and other fluid-saturated rock parameters (Goloshubin \& Korneev, 2005; V. Korneev, 2007; V. A. Korneev et al., 2004).

\subsection{Discussion}

An attempt is made to numerically represent the frequency-dependent seismic reflections from a fluid-saturated model. The scheme is expected to evaluate the fluid-related effects 
possibly caused by fluid flow in the pores of reservoirs in terms of the diffusive-viscous theory. However, on the inference that the wave propagate in vertical or nearly vertical directions in the media with lateral homogeneity and neglecting S-wave, the diffusiveviscous wave equation can be considered as an extended acoustic wave equation instead of an elastic one which can model the reflections of both P-wave and S-wave, or even the transformed wave numerically. By virtue of the anelastic properties of the media through which the waves propagate, the propagating waves will decay. Goloshubin and Korneev (2000, 2005); Goloshubin et al. (2002); V. A. Korneev et al. (2004); V. Korneev (2007) researched into and obtained from laboratory experiments the diffusive attenuation parameter $(\zeta)$ and viscous attenuation parameter $(\eta)$ used in this thesis. The parameters previously mentioned, which absolutely, have a considerable effect on snapshots and seismograms, can be analytically acquired from the basic laws of physics or, effectively, through Amplitude Versus Offset/Angle (AVO/AVA) analysis (Goloshubin \& Korneev, 2000, 2005; Goloshubin et al., 2002; V. A. Korneev et al., 2004; V. Korneev, 2007). Particularly, the low-frequency effects related to hydrocarbon accumulations stem from a range of effects akin to the various physical characteristics and mechanisms of reservoirs. It is rather laborious (if not impracticable) to represent the seismic low-frequency anomalies by concurrently considering all the potentially associated factors in the reservoir (Goloshubin \& Korneev, 2000, 2005; Goloshubin et al., 2002; V. A. Korneev et al., 2004; V. Korneev, 2007).

The primary objective of this numerical method is to develop a finite volume scheme with the aim of understanding wave propagation in fluid-saturated media related to the diffusive and viscous effects of the pore fluid in reservoirs. The rationale for implementing the finite volume methods is by reason of its adaptability and straightforward approach when dealing with irregular grids (control volumes). Further, finite volume methods assure conservation of the variables involved. When dealing with source terms, finite volume methods generally perform better than other methods. The numerical results produced with this finite volume scheme can be applied in interpreting field data, thus, contributing to seismic imaging and seismic inversion. 


\subsection{Conclusion}

A two-dimensional finite volume scheme has been developed for modeling the propagation of seismic waves based on the diffusive-viscous theory. The diffusive-viscous wave equation-based simulation reviewed in this thesis presents waves propagating in a fluidsaturated medium applying finite volume method on a Cartesian grid. To our knowledge, it is the first time finite volume method is applied to the diffusive viscous wave equation.

Snapshots and seismograms, when correlated with the acoustic case, suggest a notable attenuation of the diffusive-viscous waves as they propagate through the fluid-saturated layer. The gas-saturated medium depicts a lower attenuating effect when correlated with the oil- and water-saturated media, thus, implying that the diffusive and viscous attenuation parameters $\zeta$ and $\eta$ have a considerable effect on the numerical results. The designed finite volume scheme has exhibited its adaptability and justifies its stability even for a larger time step than that applied in Zhao, Gao, and Zhao (2014) for the flux-correctedtransport finite difference scheme. This finite volume scheme remarkable provides an enhancement for the schemes previously developed such as the flux corrected transport finite difference method (Zhao, Gao, \& Zhao, 2014), reflectivity method (Zhao et al., 2019) and the extended reflectivity method (Zhao et al., 2017). Furthermore, the diffusive-viscous wave simulations apropos to this finite volume scheme can be adopted as a manual in frequency-dependent fluid delineation methods, in particular, LFS and fluid mobility attributes (which is not within the scope of this thesis). Consequently, the numerical scheme implemented in the modeling of seismic wave propagation must be significantly investigated with the objective of obtaining most appropriate results. Additionally, explicitly incorporating diffusive and viscous attenuation components in seismic wave numerical modeling is rudimentary for a practicable seismic imaging and data interpretation. Various comprehensive theoretical investigation and detailed experiments are required in the future as a means to broaden the understanding of frequency-dependent processes, considering that these mechanisms are not absolutely understood. 


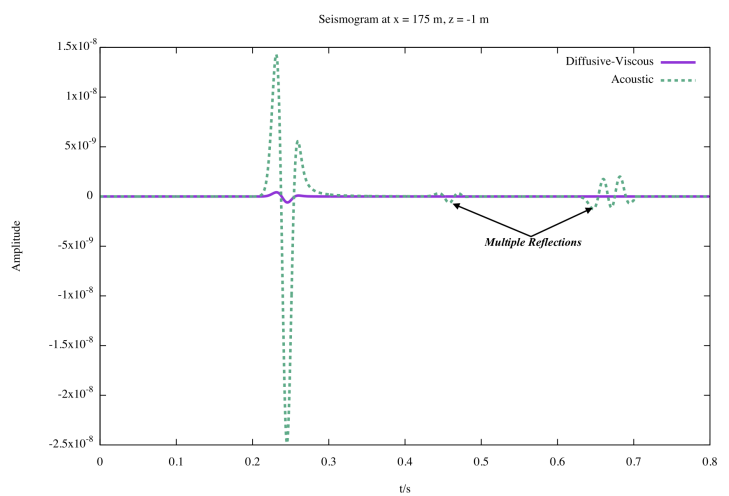

(a) Receiver A for Particle Displacement

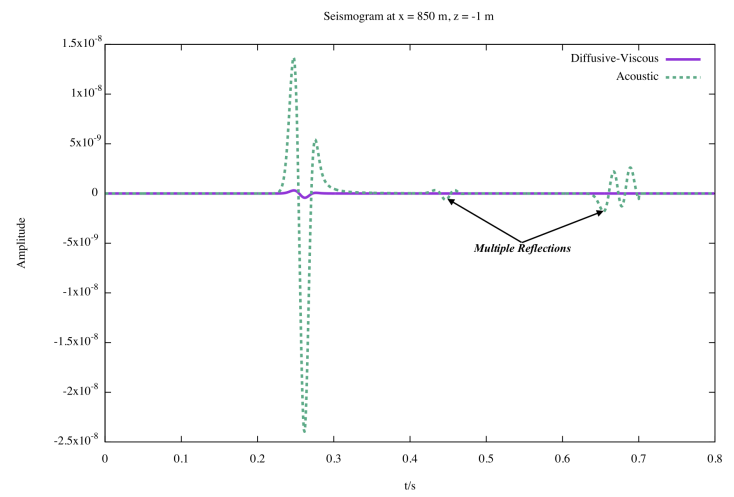

(c) Receiver C for Particle Displacement



(e) Receiver B for Particle Velocity

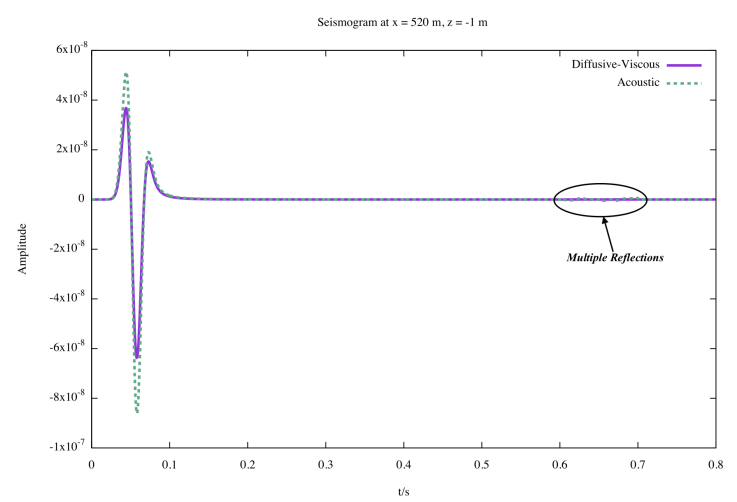

(b) Receiver B for Particle Displacement

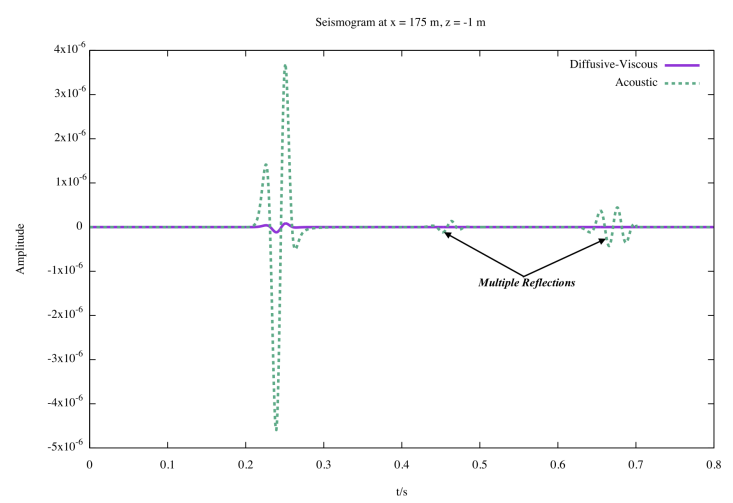

(d) Receiver A for Particle Velocity



(f) Receiver C for Particle Velocity

Figure 5.6: Seismograms generated from Particle motions (Displacement and Velocity) for diffusive-viscous wave (continuous line) compared with acoustic wave (dashed-line) for three receivers placed on the surface of the model with multiple reflections indicated, adopted from Mensah et al. (2019). 

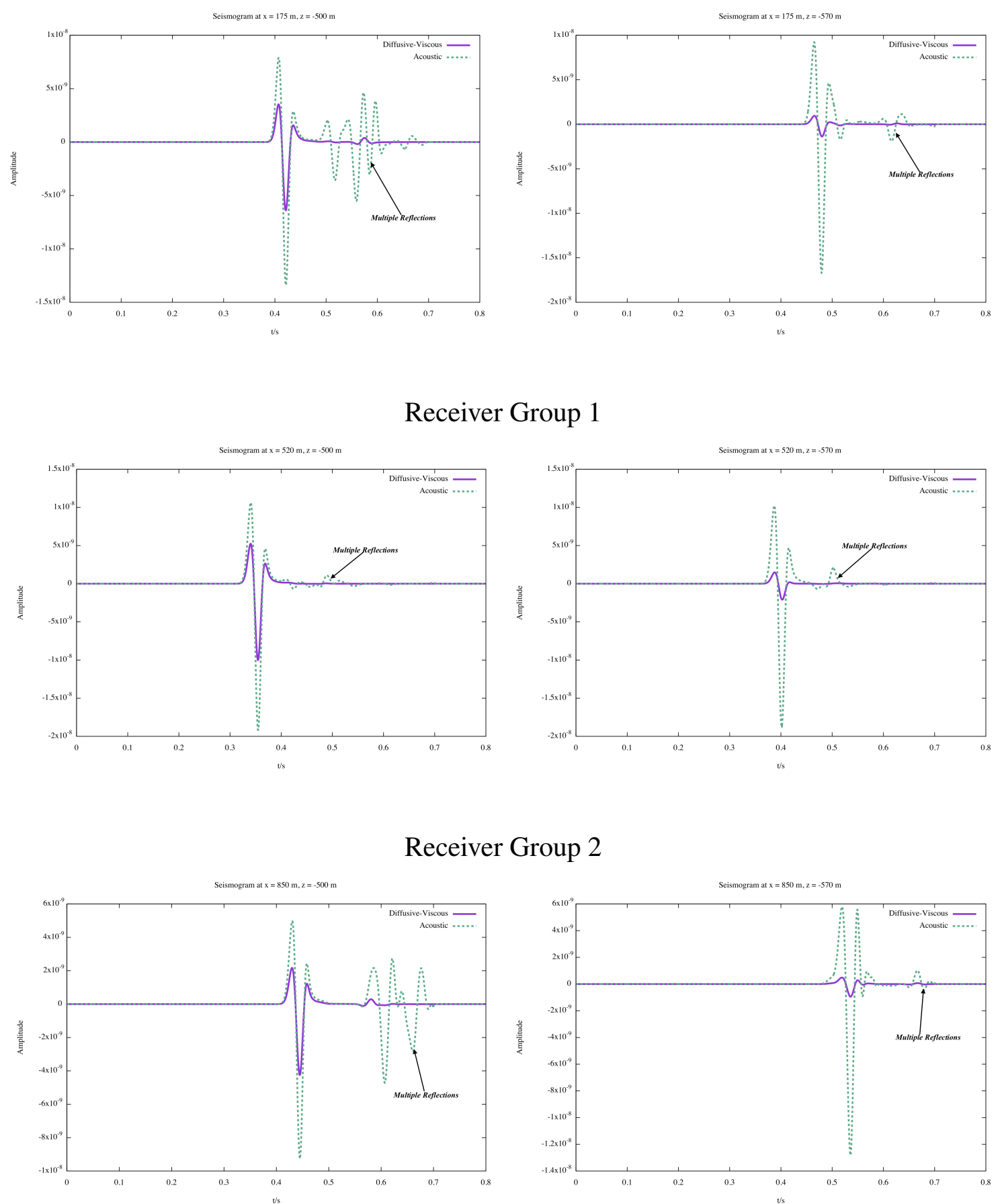

\section{Receiver Group 3}

Figure 5.7: Seismograms generated from Particle Displacement for diffusive-viscous wave (continuous line) compared with acoustic wave (dashed-line) for the set receivers with multiple reflections indicated, adopted from Mensah et al. (2019). 

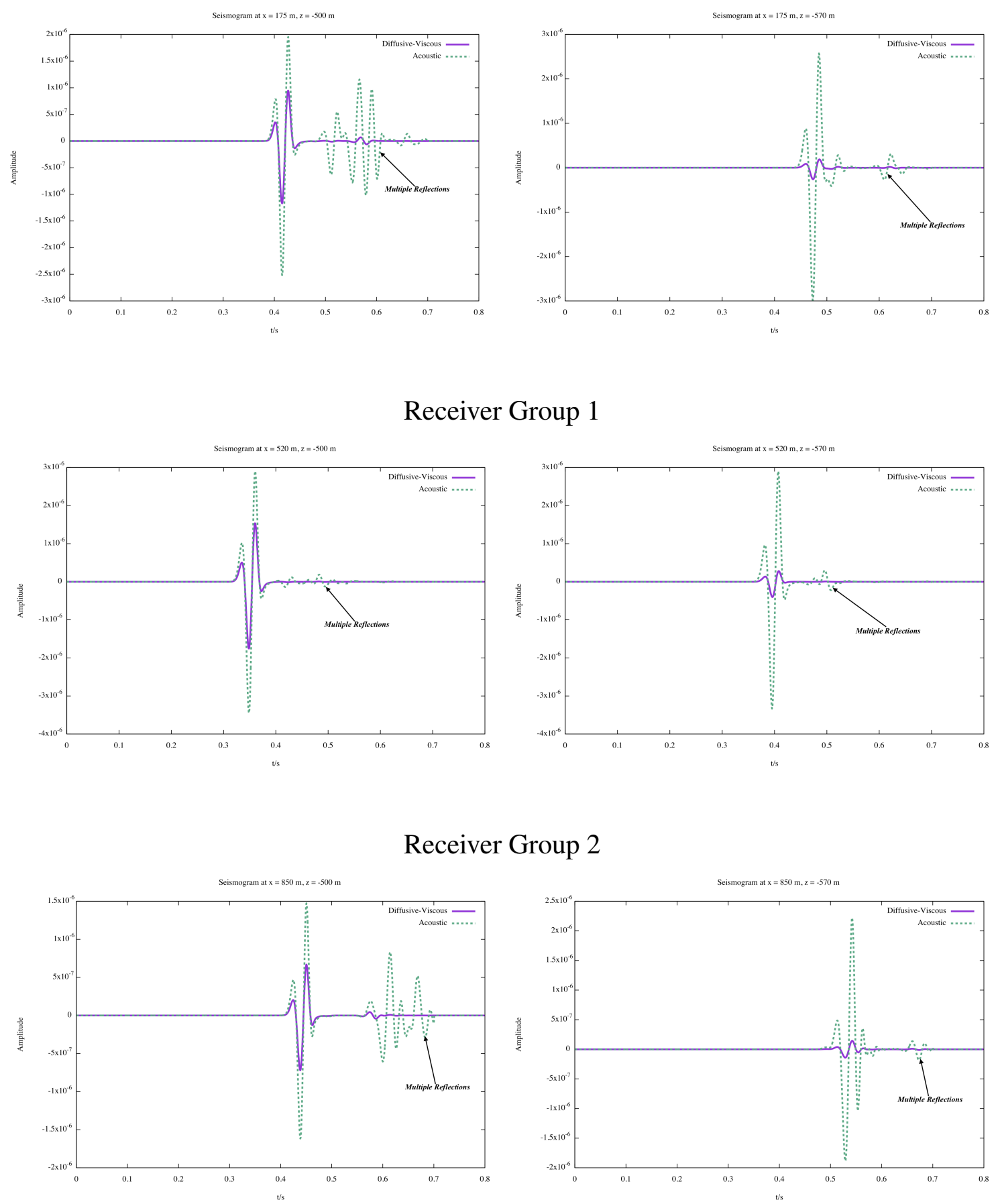

Receiver Group 3

Figure 5.8: Seismograms generated from Particle Velocity for diffusive-viscous wave (continuous line) compared with acoustic wave (dashed-line) for the set receivers with multiple reflections indicated, adopted from Mensah et al. (2019). 


\subsection{Summary}

In this Chapter, we have solved the two-dimensional diffusive and viscous wave equation (equation 2.3) applying the second-order Runge-Kutta finite volume method in a twodimensional Cartesian coordinate system $(x, y)$. Further, the so-called Mur Absorbing Boundary Condition (Clayton \& Engquist, 1977), which are absorbing-type, have also been implemented on all sides of the model with the exception of the surface, $(0,0)$, $(1000,0)$, which is a free surface, hence, free surface boundary condition has been applied. The elevations $(y)$ have been assumed to be increasing below mean sea level, that is, negatively downwards as in a real case. The grid spacing are equal in both directions, i.e., $x$ - and $y$-directions, and the time-step is regulated by the stability criteria (see Zhao, Gao, \& Zhao, 2014). In the next chapter, we extend the two-dimensional considerations of the diffusive-viscous wave equation in chapter four to three-dimension. 


\section{Chapter 6}

\section{Three-dimensional considerations}

In this Chapter, we present the details of applying the finite volume method in threedimensions. The structure of this chapter closely adopts to that of chapter four, which addressed two-dimensional problems.

\subsection{Meshing}

In this section, we review the characteristics of three-dimensional Cartesian meshes as applied to finite volume methods, complementing the discussion with that provided for the two-dimensional meshes in section 4.1

According to $\mathrm{Ma}, \mathrm{Li}$, and $\mathrm{Ma}$ (2020), Cartesian mesh is often applied as an approximate discretization for three-dimensional geometry. This type of mesh is filled with different orthogonal hexahedrons, and the boundary of meshes which part of the different substances is ladder-shaped as illustrated in Figure 4.1a. Three dimensional finite volume Cartesian mesh generators usually generate meshes of hexahedral elements. The generation of hybrid (mixed) meshes are as well possible by certain advanced mesh generators, that combine hexahedral elements with even, tetrahedral element types. In this thesis, all three-dimensional meshes produced are hexahedral. The comparison between structured and unstructured meshes demonstrated in Section 4.1 equally applies well to three dimensions. An advantage of using a structured grid is that it is tremendously space efficient, because the neighbourhood connections are determined by storage arrangement. Another merit the structured grid has over the unstructured grid is that it has an improved con- 
vergence and higher resolution (Castillo, 1991; George, 1992). For this thesis, structured hexahedral meshes are generated.

\subsection{Designing control volumes}

Finite volume discretization banks on the construction of control volumes around every node in the mesh. The control volume design process is more complex in three dimensions than in two dimensions, both in terms of implementation and visualization. The procedure can possibly be best represented by taking into consideration how sub-control volumes are formed. It is worth recalling from Section 4.2 that sub-control volumes are a pile up of elements to form control volumes around element nodes. A single control volume is made up of a number of sub-control volumes, each of which is assisted by one of the elements contributing to the central node of that control volume. In two dimensions, every rectangular or square grid is made up of exactly four sub-control volumes. In three dimensions, every hexahedral element is constituted by exactly eight sub-control volumes.

Figure 6.1 presents a schematic of a single sub-control volume within a control volume of hexahedral element, as well as its three-dimensional construction. The procedure serves as an extension of Section 4.2 for the two-dimensional case. It is obvious from Figure 6.1 that every sub-control volume as well has a hexahedral shape, with three "inner" control volume faces and three "outer" control volume faces. Those that are within the control volume and do not correspond to any boundary are referred to as the "inner" faces, whilst those that separate adjacent control volumes and are bounded by lines that connect the centroid of the element, the centroid of the face and the midpoints of the edges are referred to as the "outer" faces.

Every sub-control volume has a quadrilateral shape. In three dimensions, the derived control volumes are likely to be much more complicated than in two-dimensions. This is due to the fact that, firstly, each sub-control volume within every control volume has three control volume faces in three-dimensions and secondly, in three-dimensions, a given node might be shared by several other elements. 


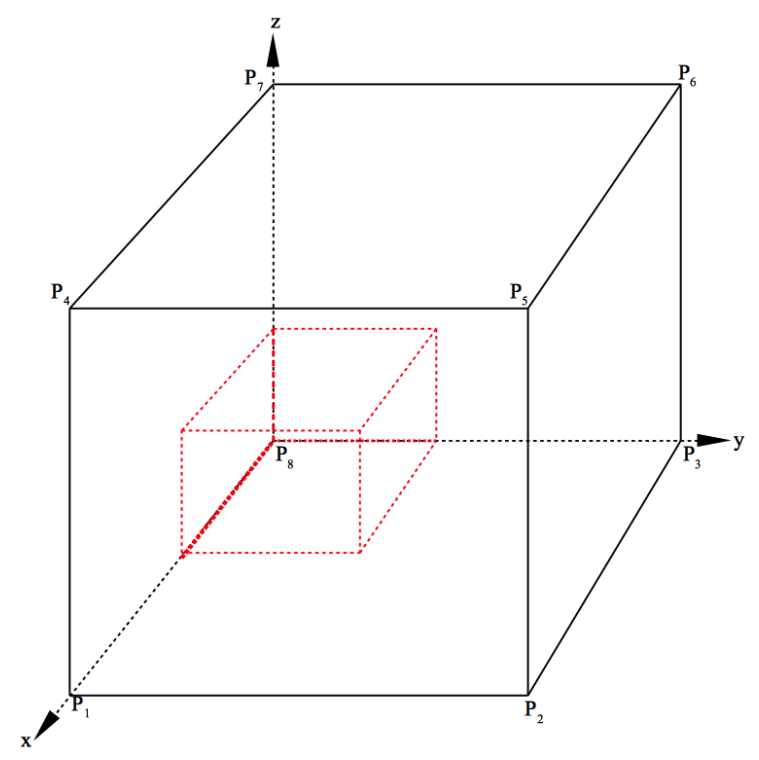

Figure 6.1: A three-dimensional sub-control volume within a control volume.

Figure 6.2 depicts several control volumes, with a few boundary control volumes fitting together. Each of the control volume's face either shares a face with another control volume in a mesh, or rests on a boundary. The finite volume technique requires evaluating fluxes through each of the control volume faces, thus, evaluating the net flow throughout each and every control volume within the mesh.

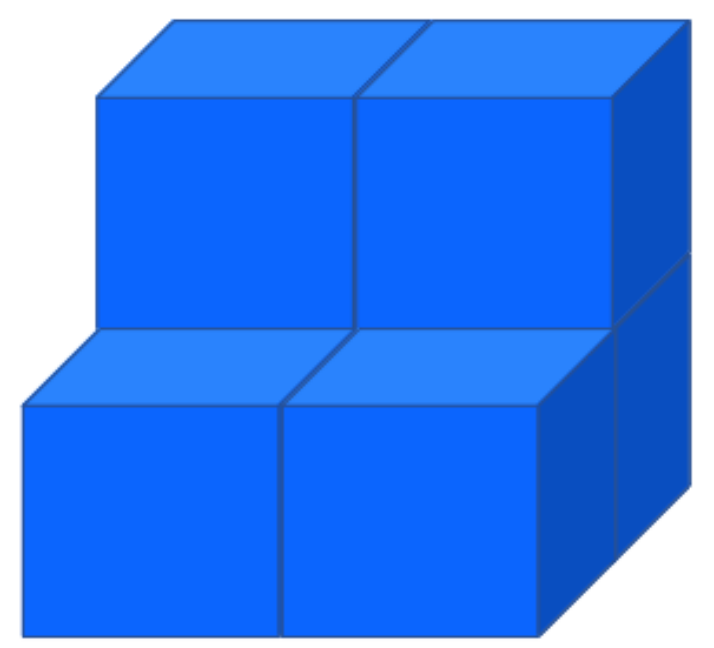

Figure 6.2: Three-dimensional control volumes. 


\subsection{Conventions}

Again, it is worthy of recalling from Section 3.3.2 that to guarantee the finite volume laws of conservation is maintained at the discrete level, there must be consistency in the fluxes evaluated at the control volume faces in the context of adjacent control volumes. In this section, the application in guaranteeing that this consistency is satisfied in threedimensions are reviewed.

A hexahedral control volume consists of eight node, six faces and eight sub-control volumes. To ensure an ease with correlating nodes, faces, and sub-control volumes with each other, a schematic of a simple notation scheme is illustrated in Figure 6.3. Control volume nodes (vertexes) and faces are denoted " $P_{1}$ " to " $P_{8}$ ", with the sub-control volume nodes and faces labelled "A" to "G". We remark here that one control volume and one sub-control volume are displayed for the sake of readability.

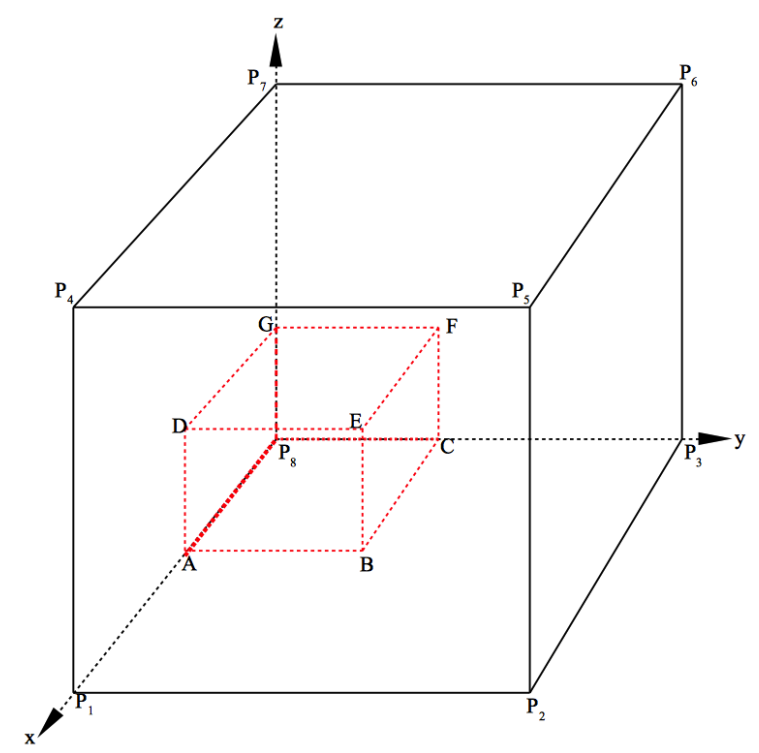

Figure 6.3: Notation convention for nodes, faces, and sub-control volumes.

In three-dimensions, there is no straightforward convention that guarantees a singular processing of every control volume face. Recall that applying an anticlockwise arrangement in two dimensions (see Figure 4.8) guarantees the flux responsibility of each sub-control volume through a single control volume face. However, such a convention is impossible in three-dimensions, since every control volume has a total of six control volume faces, 
with just four sub-control volumes. It is worthwhile to critically distribute the obligations of these six control volume faces amongst the sub-control volumes to guarantee that each and every face is treated exactly once.

The boundary faces are treated in the same manner as in two-dimensions: every subcontrol volume has a responsibility to verify if any of its "inner" faces rests on a boundary, and if it does, evaluate the fluxes through that boundary also. This verification is virtually carried out only once, during the control volume construction period. At this period, the inner faces are allocated to boundary control volumes, including whatsoever has been allocated to the outer faces applying the above discussed convention.

\subsection{Integration}

In Section 3.2, the approximation of the diffusive flux, viscous flux, velocity flux, and source term components of equation 3.12 applying numerical integration method were reviewed. The technicalities relating to these integration scheme are now reviewed for three-dimensional cases.

Figure 6.4 illustrates a single hexahedral control volume, and as was implemented in equations 3.19 and 3.20, the integral on all sides of the control volume's surface may be disintegrated into integrals over each of its faces.

$$
\begin{gathered}
\iint_{\varsigma_{i}}(\eta \nabla v \cdot \hat{\mathbf{n}}) d \varsigma=\sum_{\kappa=1}^{N f_{i}} \iint_{\varsigma_{i \kappa}}(\eta \nabla v \cdot \hat{\mathbf{n}}) d \varsigma, \\
\iint_{\varsigma_{i}}\left(\nu^{2} \nabla u \cdot \hat{\mathbf{n}}\right) d \varsigma=\sum_{\kappa=1}^{N f_{i}} \iint_{\varsigma_{i \kappa}}\left(\nu^{2} \nabla u \cdot \hat{\mathbf{n}}\right) d \varsigma,
\end{gathered}
$$






Figure 6.4: A single hexahedral control volumes.

\subsubsection{Midpoint rule (one-point Gaussian quadrature)}

These surface integrals of the viscous and velocity fluxes may be evaluated anew applying the midpoint rule

$$
\begin{gathered}
\iint_{\varsigma_{i \kappa}}(\eta \nabla v \cdot \hat{\mathbf{n}}) d \varsigma \approx A\left(\varsigma_{i \kappa}\right)[\eta \nabla v \cdot \hat{\mathbf{n}}]_{\mathbf{m}_{\mathbf{i} \kappa}}, \\
\iint_{\varsigma_{i \kappa}}\left(\nu^{2} \nabla u \cdot \hat{\mathbf{n}}\right) d \varsigma \approx A\left(\varsigma_{i \kappa}\right)\left[\nu^{2} \nabla u \cdot \hat{\mathbf{n}}\right]_{\mathbf{m}_{\mathbf{i} \kappa}},
\end{gathered}
$$

where $A\left(\varsigma_{i \kappa}\right)$ represents the area of the face, $\varsigma_{i \kappa}$. This results in the approximation

$$
\begin{gathered}
\iint_{\varsigma_{i \kappa}}(\eta \nabla v \cdot \hat{\mathbf{n}}) d \varsigma \approx \sum_{\kappa=1}^{N f_{i}} A\left(\varsigma_{i \kappa}\right)[\eta \nabla v \cdot \hat{\mathbf{n}}]_{\mathbf{m}_{\mathbf{i} \kappa}}, \\
\iint_{\varsigma_{i \kappa}}\left(\nu^{2} \nabla u \cdot \hat{\mathbf{n}}\right) d \varsigma \approx \sum_{\kappa=1}^{N f_{i}} A\left(\varsigma_{i \kappa}\right)\left[\nu^{2} \nabla u \cdot \hat{\mathbf{n}}\right]_{\mathbf{m}_{\mathbf{i} \kappa}} .
\end{gathered}
$$

Approximating the diffusive flux and source term components of equation 3.12 requires evaluating the volume $\Delta V_{i}$. To achieve this, the volumes of each of the sub-control volumes of $V_{i}$ must be summed up. Every sub-control volume is hexahedral in shape and the volume of each of these sub-control volumes may be evaluated by degenerating it into six pyramids with a common arbitrary vertex situated within the hexahedron. Davies and 
Salmond (1985) provides the formula for the computation of the volume of this pyramid as

$$
S C V=\frac{1}{12}\left(s_{1}+s_{2}\right) \cdot\left[\left(a_{1}+b_{1}\right) \times\left(b_{2}-b_{1}\right)\right]
$$

\subsubsection{Gaussian quadrature}

Gaussian quadrature can be employed in evaluating the diffusive flux, viscous flux, velocity flux, and source components across a control volume face, however a two-dimensional Gaussian quadrature is required. This method was employed in Section 4.4.2 to evaluate two dimensional volume integrals, and it is employed in three dimensions to evaluate surface integrals. Equation 4.29 is still valid, and once more the configuration

$$
\begin{aligned}
\mathbf{x}(\alpha, \beta) & =\frac{1}{4}(1-\alpha)(1-\beta) \mathbf{G}_{\mathbf{1}}+\frac{1}{4}(1+\alpha)(1-\beta) \mathbf{G}_{\mathbf{2}} \\
& +\frac{1}{4}(1+\alpha)(1+\beta) \mathbf{G}_{\mathbf{3}}+\frac{1}{4}(1-\alpha)(1+\beta) \mathbf{G}_{\mathbf{4}} \\
& -1 \leq \alpha \leq 1, \quad-1 \leq \beta \leq 1 .
\end{aligned}
$$

is implemented, with $\mathbf{G}_{\mathbf{i}}$ representing the vertices, and the Jacobian of the transformation described by

$$
\left|\frac{\partial \mathbf{x}}{\partial(\alpha, \beta)}\right|=\left\|\frac{\partial \mathbf{x}}{\partial \alpha} \times \frac{\partial \mathbf{x}}{\partial \beta}\right\| .
$$

The application of the four-point Gaussian quadrature (see Davis \& Rabinowitz, 1956; Love, 1966) in two dimensions for the viscous and velocity fluxes yields

$$
\begin{aligned}
\iint_{\varsigma_{i \kappa}}(\eta \nabla v \cdot \hat{\mathbf{n}}) d \varsigma & \approx[\eta \nabla v \cdot \hat{\mathbf{n}}]_{\mathbf{x}_{\left(\mathbf{x}_{\mathbf{1}} \gamma_{\mathbf{1}}\right)}}\left\|\frac{\partial \mathbf{x}}{\partial \alpha} \times \frac{\partial \mathbf{x}}{\partial \beta}\right\|_{\left(x_{1} \gamma_{1}\right)} \\
& +[\eta \nabla v \cdot \hat{\mathbf{n}}]_{\mathbf{x}_{\left(\mathbf{x}_{\mathbf{1}} \gamma_{\mathbf{2}}\right)}}\left\|\frac{\partial \mathbf{x}}{\partial \alpha} \times \frac{\partial \mathbf{x}}{\partial \beta}\right\|_{\left(x_{1} \gamma_{2}\right)} \\
& +[\eta \nabla v \cdot \hat{\mathbf{n}}]_{\mathbf{x}_{\left(\mathbf{x}_{\mathbf{2}} \gamma_{\mathbf{1}}\right)}}\left\|\frac{\partial \mathbf{x}}{\partial \alpha} \times \frac{\partial \mathbf{x}}{\partial \beta}\right\|_{\left(x_{2} \gamma_{1}\right)} \\
& +[\eta \nabla v \cdot \hat{\mathbf{n}}]_{\mathbf{x}_{\left(\mathbf{x}_{\mathbf{2}} \gamma_{2}\right)}}\left\|\frac{\partial \mathbf{x}}{\partial \alpha} \times \frac{\partial \mathbf{x}}{\partial \beta}\right\|_{\left(x_{2} \gamma_{2}\right)}
\end{aligned}
$$




$$
\begin{aligned}
\iint_{\varsigma_{i \kappa}}\left(\nu^{2} \nabla u \cdot \hat{\mathbf{n}}\right) d \varsigma & \approx\left[\nu^{2} \nabla u \cdot \hat{\mathbf{n}}\right]_{\mathbf{x}_{\left(\mathbf{x}_{\mathbf{1}} \gamma_{\mathbf{1}}\right)}}\left\|\frac{\partial \mathbf{x}}{\partial \alpha} \times \frac{\partial \mathbf{x}}{\partial \beta}\right\|_{\left(x_{1} \gamma_{1}\right)} \\
& +\left[\nu^{2} \nabla u \cdot \hat{\mathbf{n}}\right]_{\mathbf{x}_{\left(\mathbf{x}_{\mathbf{1}} \gamma_{\mathbf{2}}\right)}}\left\|\frac{\partial \mathbf{x}}{\partial \alpha} \times \frac{\partial \mathbf{x}}{\partial \beta}\right\| \|_{\left(x_{1} \gamma_{2}\right)} \\
& +\left[\nu^{2} \nabla u \cdot \hat{\mathbf{n}}\right]_{\mathbf{x}_{\left(\mathbf{x}_{\mathbf{2}} \gamma_{\mathbf{1}}\right)}}\left\|\frac{\partial \mathbf{x}}{\partial \alpha} \times \frac{\partial \mathbf{x}}{\partial \beta}\right\| \|_{\left(x_{2} \gamma_{1}\right)} \\
& +\left[\nu^{2} \nabla u \cdot \hat{\mathbf{n}}\right]_{\mathbf{x}_{\left(\mathbf{x}_{\mathbf{2}} \gamma_{\mathbf{2}}\right)}}\left\|\frac{\partial \mathbf{x}}{\partial \alpha} \times \frac{\partial \mathbf{x}}{\partial \beta}\right\| \|_{\left(x_{2} \gamma_{2}\right)} .
\end{aligned}
$$

The diffusive flux and source term of equation 3.12 is evaluated by applying the Gaussian quadrature in three dimensions, where a total of eight function computations per control volume is required. Nevertheless, the source term is limited by the once-off cost per sub-control volume attribute, since the source is not regarded as dependent on $u$ and $v$. If indeed the source term was regarded as dependent on $u$ and $v$, then the Gaussian quadrature method would still be implementable, but its cost would now be approximately equal to the cost for the viscous and velocity fluxes.

An extension of equation 3.27 to three dimensions is described by (Burden \& Faires, 2001)

$$
\int_{-1}^{1} \int_{-1}^{1} \int_{-1}^{1} g(x, \gamma, \chi) d \alpha d \beta \approx \sum_{j=1}^{n} \sum_{k=1}^{n} \sum_{l=1}^{n} w_{j} w_{k} w_{l} g\left(x_{j}, \gamma_{k}, \chi_{l}\right)
$$

with the weights, $w_{i}$ and abscissas, $x_{i}$ still provided by Davis and Rabinowitz (1956); Love (1966). A mapping of each sub-control volume to $[-1,1] \times[-1,1] \times[-1,1]$ is required in order to apply equation 6.12 to the diffusive flux and source term components. The following configuration which involves the sub-control vertices, say $\mathbf{P}_{\mathbf{i}}$, can be used 
to achieve this (refer to Figure 6.3)

$$
\begin{aligned}
\mathbf{x}(\alpha, \beta) & =\frac{1}{4}(1-\alpha)(1-\beta)(1-\tau) \mathbf{P}_{\mathbf{1}}+\frac{1}{4}(1+\alpha)(1-\beta)(1-\tau) \mathbf{P}_{\mathbf{2}} \\
& +\frac{1}{8}(1-\alpha)(1+\beta)(1-\tau) \mathbf{P}_{\mathbf{3}}+\frac{1}{8}(1+\alpha)(1+\beta)(1-\tau) \mathbf{P}_{\mathbf{4}}, \\
& +\frac{1}{8}(1-\alpha)(1-\beta)(1+\tau) \mathbf{P}_{\mathbf{5}}+\frac{1}{8}(1+\alpha)(1-\beta)(1+\tau) \mathbf{P}_{\mathbf{6}} \\
& +\frac{1}{8}(1-\alpha)(1+\beta)(1+\tau) \mathbf{P}_{\mathbf{7}}+\frac{1}{8}(1+\alpha)(1+\beta)(1+\tau) \mathbf{P}_{\mathbf{8}}, \\
& -1 \leq \alpha \leq 1, \quad-1 \leq \beta \leq 1, \quad-1 \leq \tau \leq 1 .
\end{aligned}
$$

with the Jacobian of the transformation described by

$$
\left|\frac{\partial \mathbf{x}}{\partial(\alpha, \beta, \tau)}\right|=\frac{\partial \mathbf{x}}{\partial \alpha} \cdot \frac{\partial \mathbf{x}}{\partial \beta} \times \frac{\partial \mathbf{x}}{\partial \tau} .
$$

Applying equations 6.13 and 6.14 to equations the diffusive flux and source term components yield

$$
\begin{aligned}
\iiint_{V_{i}} \zeta \cdot v d V & =\int_{-1}^{1} \int_{-1}^{1} \int_{-1}^{1} \zeta(v(\mathbf{x}(\alpha, \beta, \tau))) \frac{\partial \mathbf{x}}{\partial \alpha} \cdot \frac{\partial \mathbf{x}}{\partial \beta} \times \frac{\partial \mathbf{x}}{\partial \tau} d \alpha d \beta d \tau \\
& \approx \sum_{j=1}^{n} \sum_{k=1}^{n} \sum_{l=1}^{n} w_{j} w_{k} w_{l} \zeta\left(v\left(\mathbf{x}\left(x_{j}, \gamma_{k}, \chi_{l}\right)\right)\right) \frac{\partial \mathbf{x}}{\partial \alpha} \cdot \frac{\partial \mathbf{x}}{\partial \beta} \times \frac{\partial \mathbf{x}}{\partial \tau}\left(x_{j}, \gamma_{k}, \chi_{l}\right)
\end{aligned}
$$

and

$$
\begin{aligned}
\iiint_{V_{i}} S d V & =\int_{-1}^{1} \int_{-1}^{1} \int_{-1}^{1} S(\mathbf{x}(\alpha, \beta, \tau)) \frac{\partial \mathbf{x}}{\partial \alpha} \cdot \frac{\partial \mathbf{x}}{\partial \beta} \times \frac{\partial \mathbf{x}}{\partial \tau} d \alpha d \beta d \tau \\
& \approx \sum_{j=1}^{n} \sum_{k=1}^{n} \sum_{l=1}^{n} w_{j} w_{k} w_{l} S\left(\mathbf{x}\left(x_{j}, \gamma_{k}, \chi_{l}\right)\right) \frac{\partial \mathbf{x}}{\partial \alpha} \cdot \frac{\partial \mathbf{x}}{\partial \beta} \times \frac{\partial \mathbf{x}}{\partial \tau}\left(x_{j}, \gamma_{k}, \chi_{l}\right)
\end{aligned}
$$

for the diffusive flux and source components, respectively. It is worthy of mentioning that, equation 4.33 is only valid if the source term component depends on space. 


\subsection{Interpolation}

\subsubsection{Piecewise constant reconstruction}

In three dimensions, the piecewise constant reconstruction scheme is closely related to the two-dimensional scheme provided in Section 4.5.1, and can be applied to compute the fluxes through the cell faces, and based on this function reconstruction model, the variable values, $u$ and $v$, at the cell faces are interpolated. The only difference is that, in Section 4.5.1, this reconstruction scheme was used in computing two-dimensional "volume" integrals, but in three dimensions, it is applied in computing "surface" integrals.

The diffusive flux and source term components of equation 3.12 can be evaluated as simply the cell averages within each cell. In particular, the piecewise constant reconstruction may be applied to compute these flux components. Equations 4.34 and 4.35 are still applied, except that they are extended to three dimensions. This produces

$$
\int_{k-\frac{1}{2}}^{k+\frac{1}{2}} \int_{j-\frac{1}{2}}^{j+\frac{1}{2}} \int_{i-\frac{1}{2}}^{i+\frac{1}{2}}[\zeta \cdot v] \mathrm{d} x \mathrm{~d} y \mathrm{~d} z \approx \zeta \cdot V_{i, j, k}
$$

and

$$
\int_{k-\frac{1}{2}}^{k+\frac{1}{2}} \int_{j-\frac{1}{2}}^{j+\frac{1}{2}} \int_{i-\frac{1}{2}}^{i+\frac{1}{2}}(s) \mathrm{d} x \mathrm{~d} y \mathrm{~d} z \approx S_{i, j, k},
$$

respectively, where $S_{i, j, k}$ and $V_{i, j, k}$ are the cell averages of $s$ and $v$ at the control volume, $i, j, k$, respectively.

\subsubsection{Piecewise linear reconstruction}

In Section 4.5.2, the piecewise linear reconstruction was discussed for the two-dimensional problem. This two-dimensional concept of equations 4.36 and 4.37 can be extended to three dimensions. In three dimensions, the piecewise linear reconstruction scheme can be applied to compute the fluxes through the cell faces, and based on this function reconstruction model, the variable values, $u$ and $v$, and their gradients, $\nabla u$ and $\nabla v$ at the cell faces are interpolated. Figure 4.11 shows the evolution of the fluxes in time and can be 
applied for the three-dimensional extension.

The integration is carried out in the $x-, y-$, and $z-$ directions over each cell volume. Figure 4.12 illustrates the piecewise linear reconstruction procedure for the variable gradients, $u$ and $v$, in one-dimension, however this idea can easily be transformed to suit the three-dimensional problem. Note that all notations in Figure 4.12 remains the same and applies to the three dimensional case as well.

The viscous flux and velocity flux components of equation 3.12 may be computed with the piecewise linear reconstruction in three dimensions. This gives

$$
\begin{aligned}
\int_{k-\frac{1}{2}}^{k+\frac{1}{2}} \int_{j-\frac{1}{2}}^{j+\frac{1}{2}} \int_{i-\frac{1}{2}}^{i+\frac{1}{2}} & {\left[\eta \nabla\left(\nabla v_{x}+\nabla v_{y}+\nabla v_{z}\right)\right] \mathrm{d} x \mathrm{~d} y \mathrm{~d} z } \\
& \approx \eta\left[\left(\frac{V_{i+1}-V_{i}}{\Delta x^{2}}\right)-\left(\frac{V_{i}-V_{i-1}}{\Delta x^{2}}\right)\right] \\
& +\eta\left[\left(\frac{V_{j+1}-V_{j}}{\Delta y^{2}}\right)-\left(\frac{V_{j}-V_{j-1}}{\Delta y^{2}}\right)\right] \\
& +\eta\left[\left(\frac{V_{k+1}-V_{k}}{\Delta z^{2}}\right)-\left(\frac{V_{k}-V_{k-1}}{\Delta z^{2}}\right)\right],
\end{aligned}
$$

and

$$
\begin{aligned}
\int_{k-\frac{1}{2}}^{k+\frac{1}{2}} \int_{j-\frac{1}{2}}^{j+\frac{1}{2}} \int_{i-\frac{1}{2}}^{i+\frac{1}{2}} & {\left[\nu^{2} \nabla\left(\nabla u_{x}+\nabla u_{y}+\nabla u_{z}\right)\right] \mathrm{d} x \mathrm{~d} y \mathrm{~d} z } \\
& \approx \nu^{2}\left[\left(\frac{U_{i+1}-U_{i}}{\Delta x^{2}}\right)-\left(\frac{U_{i}-U_{i-1}}{\Delta x^{2}}\right)\right] \\
& +\nu^{2}\left[\left(\frac{U_{j+1}-U_{j}}{\Delta y^{2}}\right)-\left(\frac{U_{j}-U_{j-1}}{\Delta y^{2}}\right)\right] \\
& +\nu^{2}\left[\left(\frac{U_{k+1}-U_{k}}{\Delta z^{2}}\right)-\left(\frac{U_{k}-U_{k-1}}{\Delta z^{2}}\right)\right],
\end{aligned}
$$

where $V_{i, j, k}$ and $U_{i, j, k}$ are cell averages of $v$ and $u$ at the control volume, $i, j, k$, respectively. The indexes $i, j$, and $k$ denote the $x-, y-$, and $z$ - directions, respectively. The velocity flux and source term components in equation 3.12 may be computed with the piecewise linear reconstruction in three dimensions as simply the cell averages over the control volume to obtain similar results as in equations (6.17) and (6.18) 


\subsubsection{Shape function}

In three dimensions, the shape function method gives rise to linear interpolants $u_{i}(x, y, z)$ and $v_{i}(x, y, z)$ over each hexahedral control volume. If the vertices of the control volume are numbered 1 to 8 in all three directions, then the shape function interpolants are described by

$$
u(x, y, z) \approx \sum_{i=1}^{8} N_{i}(x, y, z) u_{i}
$$

and

$$
v(x, y, z) \approx \sum_{i=1}^{8} N_{i}(x, y, z) v_{i} .
$$

Refer to de Souza Araujo et al. (2013, for the shape functions, $N_{i}(x, y, z)$, for a hexahedral control volumes.).

\subsection{Complete finite volume discretization}

The solution to the non-linear part of the finite volume discretization (equation 3.16) in three dimensions can now be achieved, with the methods for integration and interpolation established. In the case of function reconstruction, $f_{i}$ is given as

$$
\begin{aligned}
& f(\mathbf{u}, \mathbf{v})=-\zeta \cdot V_{i, j, k}+S_{i, j, k} \\
& +\eta\left[\left(\frac{V_{i+1}-2 V_{i}+V_{i-1}}{\Delta x^{2}}\right)+\left(\frac{V_{j+1}-2 V_{j}+V_{j-1}}{\Delta y^{2}}\right)+\left(\frac{V_{k+1}-2 V_{k}+V_{k-1}}{\Delta z^{2}}\right)\right] \\
& +\nu^{2}\left[\left(\frac{U_{i+1}-2 U_{i}+U_{i-1}}{\Delta x^{2}}\right)+\left(\frac{U_{j+1}-2 U_{j}-U_{j-1}}{\Delta y^{2}}\right)+\left(\frac{U_{k+1}-2 U_{k}-U_{k-1}}{\Delta z^{2}}\right)\right] .
\end{aligned}
$$

In the case of the shape function method, $f_{i}$, is given by

$$
\begin{aligned}
f_{i}(\mathbf{u}, \mathbf{v}) & =\sum_{\kappa=1}^{N f_{i}} A\left(\varsigma_{i \kappa}\right)\left[-\zeta \cdot V\left(\mathbf{x}_{\mathbf{i}}\right) \Delta V_{i}+\eta\left(v_{i}\left(\mathbf{m}_{\mathbf{i} \kappa}\right)\right) \nabla v_{i}\left(\mathbf{m}_{\mathbf{i} \kappa}\right) \cdot \hat{\mathbf{n}}\right] \\
& +\left[\nu^{2}\left(u_{i}\left(\mathbf{m}_{\mathbf{i} \kappa}\right)\right) \nabla u_{i}\left(\mathbf{m}_{\mathbf{i} \kappa}\right) \cdot \hat{\mathbf{n}}\right]+S\left(\mathbf{x}_{\mathbf{i}}\right) \Delta V_{i},
\end{aligned}
$$

where $u_{i}$ and $v_{i}$ are the shape function interpolants (equation 6.24), $A\left(\varsigma_{i \kappa}\right)$ represents the 
area of the face, $\varsigma_{i \kappa}$, and $\Delta V_{i}$ is the volume of the control volume, $V_{i}$, described by figure 4.8 .

\subsection{Summary}

In this Chapter, we have reviewed the three-dimensional considerations of the diffusiveviscous wave equation. We have extended the various spatial discretization techniques discussed in Chapters three and four to three dimensions. Time integration schemes have not been discussed in this Chapter. In the next Chapter, we implement the threedimensional considerations reviewed in this Chapter to a test case and we include a time integration technique. 


\section{Chapter 7}

\section{Diffusive-viscous wave modelling in three- dimensions with the simple Cartesian scheme}

In this Chapter, we investigate the effectiveness of the three-dimensional methods, in particular, the function reconstruction schemes proposed in the previous Chapter by applying it to the three-dimensional diffusive-viscous wave equation. The first section of this chapter outlines the numerical model implemented and the meshing system applied. Subsequent sections deal with the numerical results and the order of accuracy of the numerical method.

\subsection{Numerical Model}

In order to conclude on the effectiveness of the function reconstruction schemes earlier reviewed, a test is carried out exclusively on the diffusive-viscous wave equation (DVWE). It is strenuous, if not impossible, to obtain a general analytical solution for the diffusiveviscous wave equation since it contains spatial and temporal differential terms. Hence, a numerical approach is required to approximate the solution. The governing equation, Eq.(2.3), is transformed into a system of equation, written in vector components (Eq.7.1), that is, velocity-displacement formulations (Mensah et al., 2019)

$$
\frac{\partial}{\partial t} \mathbf{Q}=\mathbf{L}(\mathbf{u}, \mathbf{v})+\mathbf{S}
$$


where the vector components,

$\mathbf{Q}=\left(\begin{array}{l}u \\ v\end{array}\right) ; \mathbf{S}=\left(\begin{array}{l}0 \\ s\end{array}\right) ; \mathbf{L}=\left(\begin{array}{c}v \\ -\zeta \cdot v+\eta\left[\frac{\partial^{2} v}{\partial x^{2}}+\frac{\partial^{2} v}{\partial y^{2}}+\frac{\partial^{2} v}{\partial z^{2}}\right]+\nu^{2}\left[\frac{\partial^{2} u}{\partial x^{2}}+\frac{\partial^{2} u}{\partial y^{2}}+\frac{\partial^{2} u}{\partial z^{2}}\right]\end{array}\right)$

where we have implemented the so-called Ricker wavelet as the source term which is given by the expression,

$s(t)=\left(1-2 \pi^{2} f^{2} t^{2}\right) \exp \left(-\pi^{2} f^{2} t^{2}\right)$ just as was done in Chapter five; $f$ and $t$ are the dominant frequency and time in Hertz and seconds respectively.

We solve the system of equation over a domain which is divided into several discrete volumes, as shown in 7.1, with a representative value of each volume located at its centre using a finite volume approach upon a Cartesian mesh in three dimensions. The finite volume method (FVM) can be defined as an integral technique used to solve the spatial derivatives in the governing equations by the integration of the equations over a finite volume. With the help of Gauss' divergence theorem, the integrals are, transformed where necessary. The physical meaning is that fluxes flow through the finite volume faces while satisfying flux balance over the volume. The conserved variables, which represents the average value over each cell, are defined at each cell centre. The interfaces between the cells are also used to compute the fluxes. We use the second-order Runge-Kutta TVD (total variational diminishing) method to integrate the temporal derivative. The FVM can be duly appropriate for this problem, considering its straightforward physical interpretation, mass conservation and steep front approximations, especially in multidimensional challenges (Manzini \& Ferraris, 2004; Mensah et al., 2019).

For a three dimensional domain, the cells are numbered $(i, j, k)$ but for the sake of convenience, we employ the compass notation notable to many, which was invented by Imperial College (Patankar, 1980; Ferziger \& Peric, 2012; Mensah et al., 2019), when referring to the neighbours of a cell (see figure 7.1). The cell upon which we discretize the equations is denoted by subscript $P$, with the immediate neighbours in the $x$-axis indicated by $E$ and $W$ (for East and West), $N$ and $S$ (for North and South) in the $y-$ axis and $T$ and $B$ (Top and Bottom) in the $z$-axis. The neighbouring cell center is denoted by a capital letter, while a lower case denotes a value at the cell interface. 


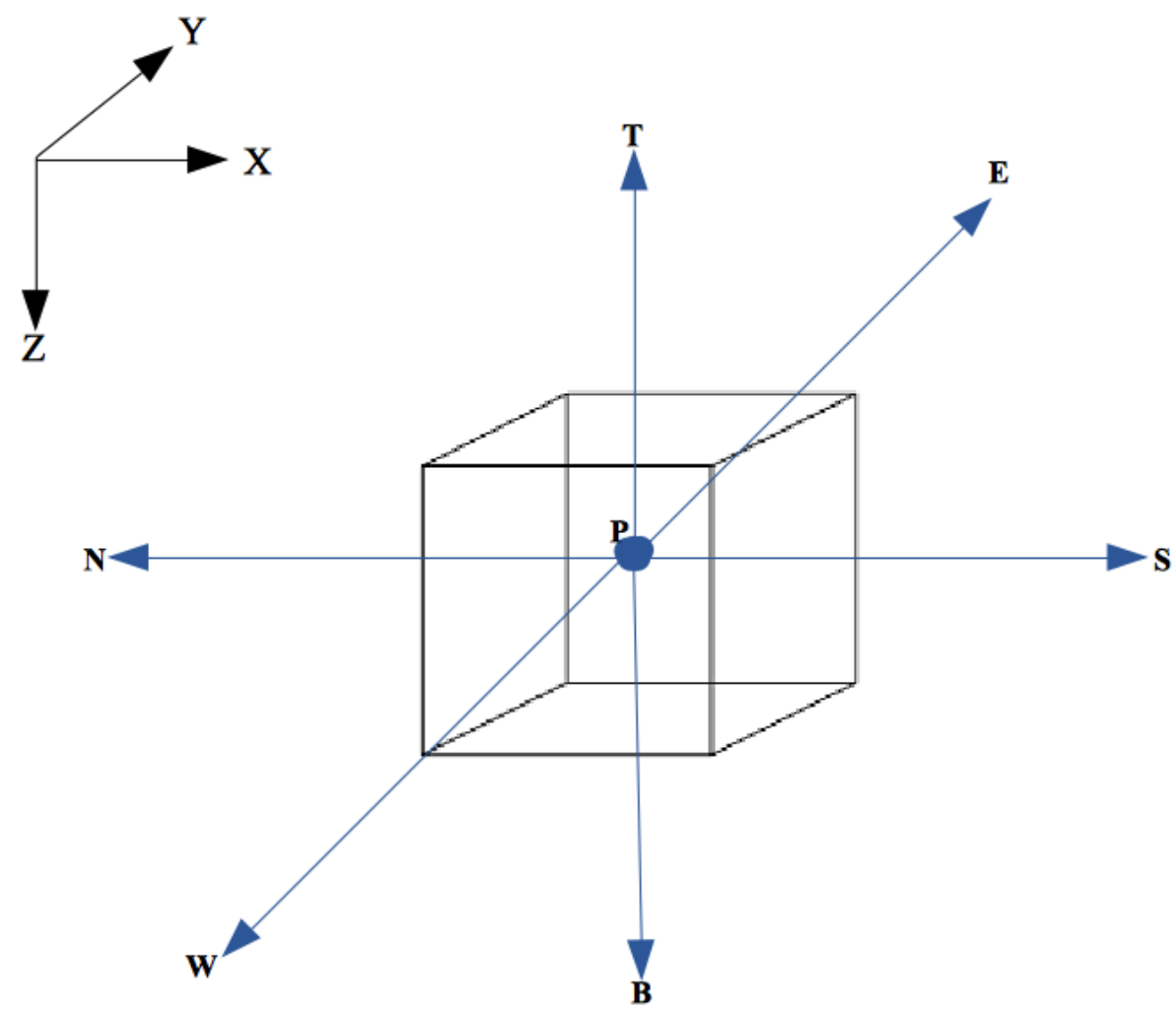

Figure 7.1: The discretisation of a 3-D domain into Cartesian finite volumes.

We construct a semi-discrete finite volume (FV) scheme for the system of equation in equation (7.1). To obtain an FV discretisation to the equation (7.1), equation (7.1) is integrated on a three-dimensional mesh using cell averages in the $x-, y-$ and $z$-axis over the cell volume. We describe the flux reconstruction procedure for both solutions in Figure (4.12) for a one-dimensional case. Still, this idea can be, transformed easily to suit the three-dimensional problem, and it is according to the above figures that the integration is carried out. The semi-discrete form of equation (7.1) is given as (Mensah et al., 2019)

$$
\frac{d}{d t} \mathbf{Q}_{P}=\mathbf{L}_{P}(u(t), v(t))+\boldsymbol{\Gamma}(t)
$$

where $\mathrm{Q}_{P}$ represents the cell average of the vector of unknowns, $\mathrm{Q}$ in the control volume, $P$ and $\mathbf{L}_{P}(u(t), v(t))$ is the cell average of the operator appearing on the RHS of equation (7.1) over the control volume, $P$. This operator is a vector with two components, say 
$\mathbf{L}_{P}=\left(\left(L_{P}\right)_{1},\left(L_{P}\right)_{2}\right)^{\top}$, the first of them given as the simple expression

$$
\left(L_{P}\right)_{1}=V_{P}(t)
$$

where $V_{P}(t)$ is the cell average of the function $v$ over the control volume $P$. The second component of the operator is obtained as

$$
\begin{aligned}
& \left(L_{P}\right)_{2}=-\frac{\zeta}{\Delta x \Delta y \Delta z} \int_{b}^{t} \int_{s}^{n} \int_{w}^{e} v d x d y d z \\
& +\frac{\eta}{\Delta x \Delta y \Delta z} \int_{b}^{t} \int_{s}^{n} \int_{w}^{e}\left[\frac{\partial}{\partial x}\left(\frac{\partial v}{\partial x}\right)+\frac{\partial}{\partial y}\left(\frac{\partial v}{\partial y}\right)+\frac{\partial}{\partial z}\left(\frac{\partial v}{\partial z}\right)\right] d x d y d z \\
& +\frac{\nu^{2}}{\Delta x \Delta z} \int_{b}^{t} \int_{s}^{n} \int_{w}^{e}\left[\frac{\partial}{\partial x}\left(\frac{\partial u}{\partial x}\right)+\frac{\partial}{\partial y}\left(\frac{\partial u}{\partial y}\right)+\frac{\partial}{\partial z}\left(\frac{\partial u}{\partial z}\right)\right] d x d y d z
\end{aligned}
$$

We approximate the second derivative terms, that is, the viscous and velocity fluxes, with a piecewise linear reconstruction as three nested central difference approximations.

$$
\begin{aligned}
& \left(L_{P}\right)_{2}=-\zeta \cdot V_{P}(t)+\eta\left[\left(\left(\frac{V_{E}(t)-V_{P}(t)}{\Delta x^{2}}\right)-\left(\frac{V_{P}(t)-V_{W}(t)}{\Delta x^{2}}\right)\right)\right. \\
+ & \left.\left(\left(\frac{V_{N}(t)-V_{P}(t)}{\Delta y^{2}}\right)-\left(\frac{V_{P}(t)-V_{S}(t)}{\Delta y^{2}}\right)\right)+\left(\left(\frac{V_{T}(t)-V_{P}(t)}{\Delta z^{2}}\right)-\left(\frac{V_{P}(t)-V_{B}(t)}{\Delta z^{2}}\right)\right)\right] \\
+ & \nu^{2}\left[\left(\left(\frac{U_{E}(t)-U_{P}(t)}{\Delta x^{2}}\right)-\left(\frac{U_{P}(t)-U_{W}(t)}{\Delta x^{2}}\right)\right)+\left(\left(\frac{U_{N}(t)-U_{P}(t)}{\Delta y^{2}}\right)-\left(\frac{U_{P}(t)-U_{S}(t)}{\Delta y^{2}}\right)\right)\right. \\
+ & \left.\left(\left(\frac{U_{T}(t)-U_{P}(t)}{\Delta z^{2}}\right)-\left(\frac{U_{P}(t)-U_{B}(t)}{\Delta z^{2}}\right)\right)\right]
\end{aligned}
$$

We apply the commonly used method of lines in solving PDEs, which has to do with initially discretising only the spatial derivatives that result in a system of ODEs, namely equation (7.2) with equations (7.3) and (7.5), for which an appropriate ODE solver must be implemented. A second-order solver for time integration should be applied since the space approximation is built using the piecewise linear reconstruction of second-order accuracy. In this thesis, we apply a second-order Runge-Kutta TVD scheme (refer to 
Shu \& Osher, 1988; Gottlieb \& Shu, 1998, for details on Runge-Kutta TVD schemes). Even though other methods can be applied such as the MUSCL-Hancock scheme that has second-order of accuracy both in space and time. The expressions for the second-order Runge-Kutta TVD scheme (refer to Shu \& Osher, 1988; Mensah et al., 2019) are

$$
\begin{aligned}
Q_{P}^{(1)} & =Q_{P}^{n}+\Delta t \cdot \mathbf{L}_{P}\left(Q_{P}^{n}\right), \\
Q_{P}^{n+1} & =\frac{1}{2} Q_{P}^{n}+\frac{1}{2} Q_{P}^{(1)}+\frac{1}{2} \Delta t \cdot \mathbf{L}_{P}\left(Q_{P}^{(1)}\right) .
\end{aligned}
$$

We integrate the source term, which is the third term in Eq.(2.20) as

$$
\boldsymbol{\Gamma}(t)=\frac{1}{\Delta x \Delta y \Delta z} \int_{b}^{t} \int_{s}^{n} \int_{w}^{e} s(t) d x d y d z=s(t)
$$

where $\Gamma(t)$ represents the cell average of the source term. Reference is made here to the second equation in Eq.(5.18) since the source term is zero in the first equation.

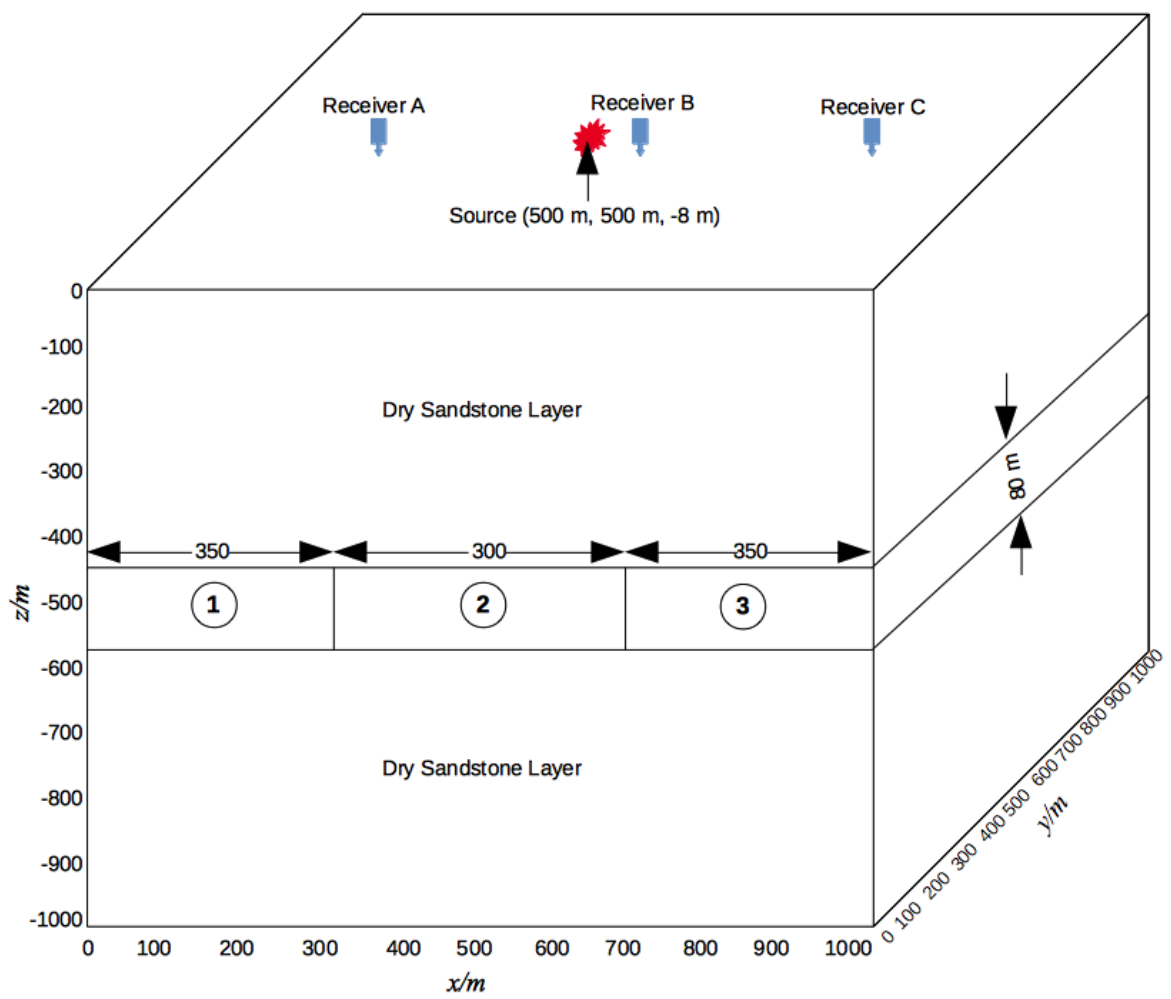

Figure 7.2: The fluid-saturated model with an $80 \mathrm{~m}$ thick (1)oil-, (2)water- and (3)gassaturated layer embedded in a homogeneous half space. 


\subsection{Validation of the numerical scheme}

The aim of this section is to assess the accuracy of the numerical scheme used in this work, which is based on a finite volume scheme with piecewise linear reconstruction with a second order RK-TVD scheme for time integration. In order to proceed, we propose the following problem, with known analytical solution

$$
\left\{\begin{array}{l}
\frac{\partial v}{\partial t}=-\zeta v+\eta \Delta v+\nu^{2} \Delta u+S, \forall(x, y, z) \in\left(-\frac{\pi}{2}, \frac{\pi}{2}\right), \forall t>0 \\
\frac{\partial u}{\partial t}=v, \forall(x, y, z) \in\left(-\frac{\pi}{2}, \frac{\pi}{2}\right), \forall t>0 \\
\left.\frac{\partial u}{\partial \mathbf{n}}\right|_{\Gamma}=\left.\frac{\partial v}{\partial \mathbf{n}}\right|_{\Gamma}=0, \forall t>0 \\
u(x, y, z, 0)=v(x, y, z, 0)=0, \forall(x, y, z) \in\left(-\frac{\pi}{2}, \frac{\pi}{2}\right),
\end{array}\right.
$$

where $\Gamma$ is the boundary, $u=u(x, y, z, t), v=v(x, y, z, t)$, and $S=S(x, y, z, t)$ being

$$
S=S(x, y, z, t)=2 P^{2}+\eta v(x, y, z, t)-2 t\left(Q-P^{2}\right)\left(2 \eta+t \nu^{2}\right)
$$

where we have denoted as $P=\sin (x)+\sin (y)+\sin (z), Q=\cos ^{2}(x)+\cos ^{2}(y)+\cos ^{2}(z)$. Since we are using Cartesian coordinates the laplacians can be written as $\Delta v=\frac{\partial v}{\partial x^{2}}+$ $\frac{\partial v}{\partial y^{2}}+\frac{\partial v}{\partial z^{2}}$ and $\Delta u=\frac{\partial u}{\partial x^{2}}+\frac{\partial u}{\partial y^{2}}+\frac{\partial u}{\partial z^{2}}$.

The exact solution of (7.8) is given by

$$
\begin{aligned}
& u(x, y, z, t)=t^{2}(\sin (x)+\sin (y)+\sin (z))^{2}, \\
& v(x, y, z, t)=2 t(\sin (x)+\sin (y)+\sin (z))^{2} .
\end{aligned}
$$

We remark that the problem, equation (7.8), has the main features of the diffusive-viscous wave model studied in this work, but with an additional source term $S$ in order to have an exact solution which allows to perform the validation of the numerical scheme. We show, in figure 7.3, that the displacement is obtained by solving the problem, equation (7.8), by means of the proposed numerical scheme. The result depicted in this figure represents the displacement, $u(x, y, z, t)$, when $y=0$ and for an output time $t=0.1$, that is $u(x, 0, z, 0.1)$. The mesh used is composed of $50 \times 50 \times 50$ cells. 


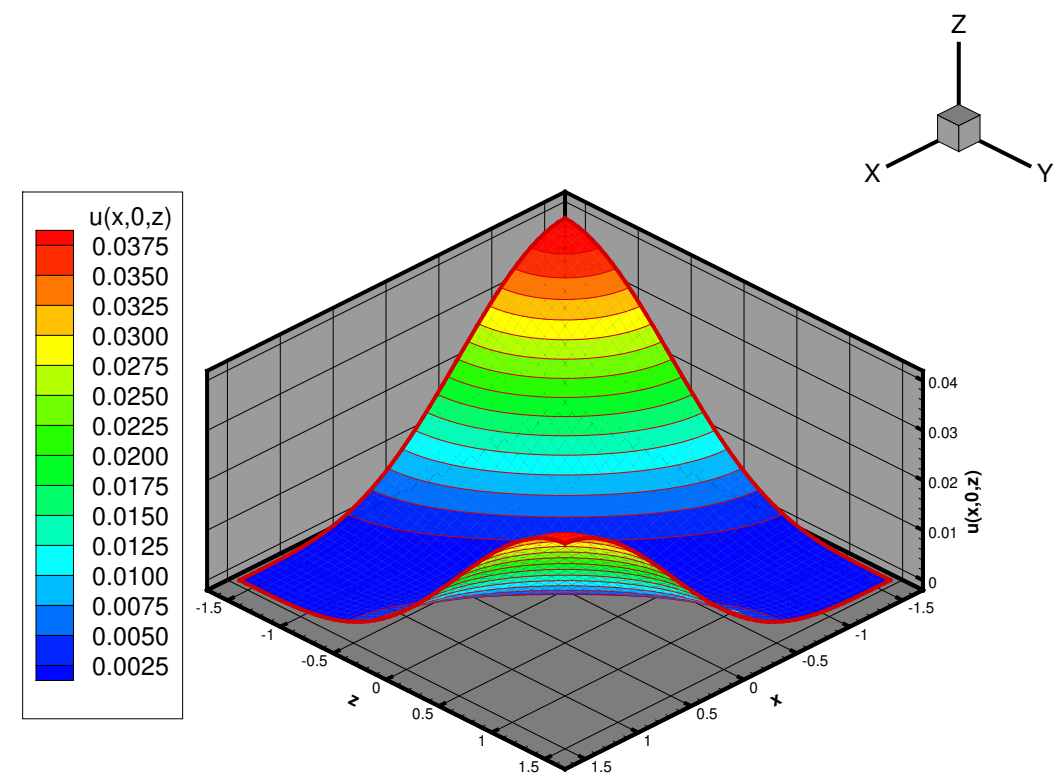

Figure 7.3: Representation of the displacement, $u(x, 0, z, 0.1)$. Cut with the plane $\mathrm{y}=0$. Output time $=0.1$ 


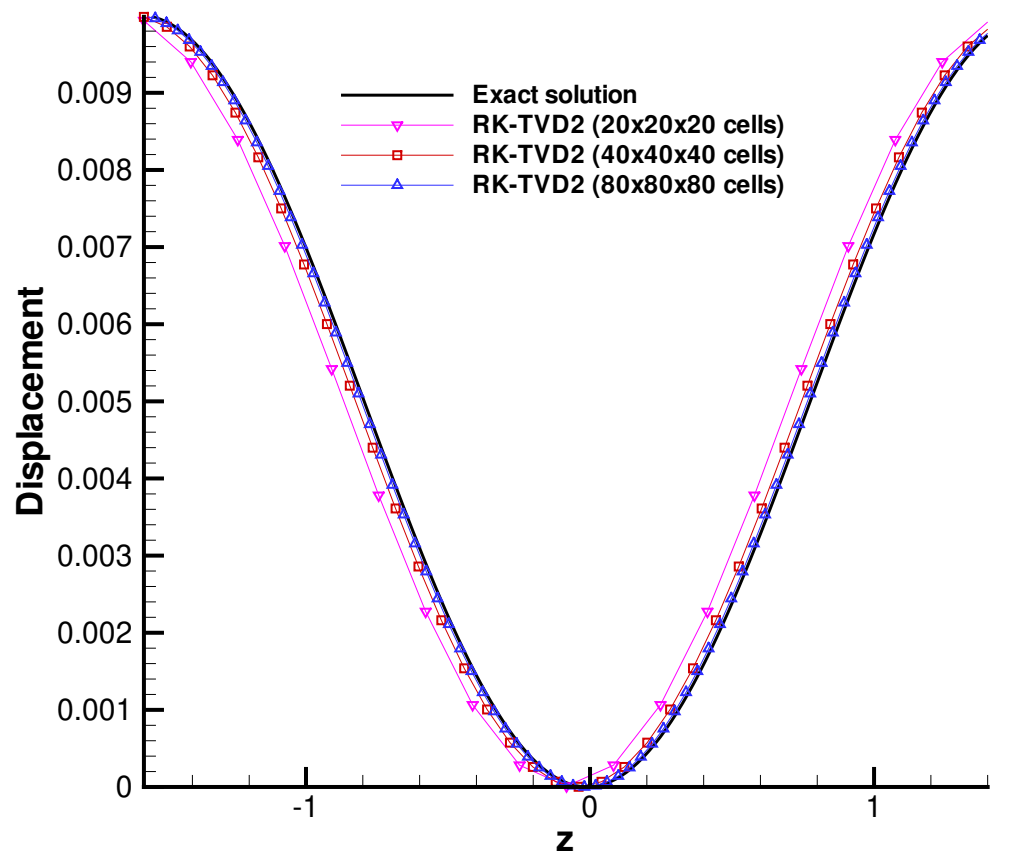

Figure 7.4: Comparison of the numerical solution of the problem Eq.(7.8) for the displacement $u(0,0, z, 0.1)$ with the exact solution given by Eq.(7.10) for different meshes. Cut with the planes $\mathrm{x}=0$., $\mathrm{y}=0$.

In order to show the convergence of the proposed numerical scheme we display, in figures 7.4 and 7.5, the numerical results obtained by taking several meshes for the problem equation (7.8) and the exact solution given by equation (7.10), for the output time $t_{\max }=0.1$. The $3-D$ solution is depicted cutting with the planes $x=0$ and $y=0$.

In figure 7.5, we carry out the comparison of the numerical solution, using several meshes, with the exact one, always referred to the problem equation (7.8) and cutting the $3-D$ plot with the planes $x=0$ and $y=0$.

Both figures clearly show that the numerical solution converge to the exact solution. The next step is to verify that the order of accuracy coincides with the theoretically expected (namely second order). In order to do so, we consider several meshes, with $10 \times 10 \times 10$; $20 \times 20 \times 20 ; 40 \times 40 \times 40 ; 80 \times 80 \times 80$ and $160 \times 160 \times 160$ cells respectively 


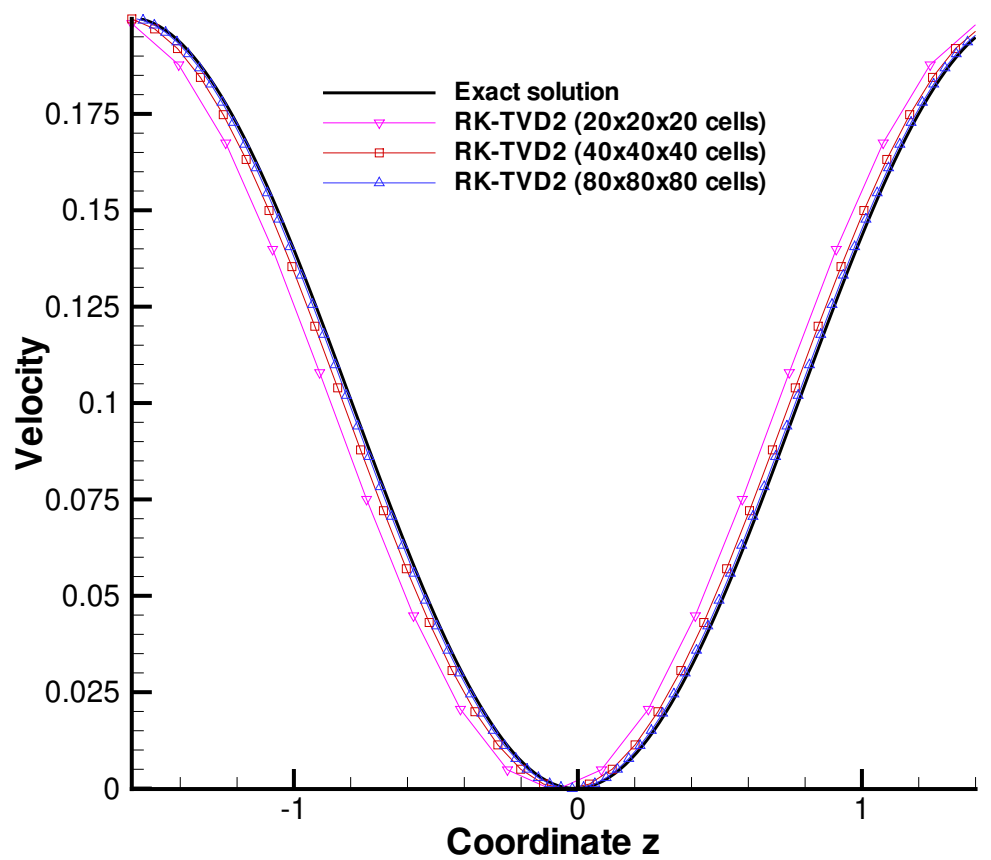

Figure 7.5: Comparison of the numerical solution of the problem equation (7.8) for the velocity $v(0,0, z, 0.1)$ with the exact solution given by equation (7.10) for different meshes. Cut with the planes $\mathrm{x}=0 ., \mathrm{y}=0$. 


\begin{tabular}{|c|c|c|}
\hline Cells & $\|$ Error $\|_{2}$ & Order \\
\hline $10 \times 10 \times 10$ & $1.08 \times 10^{-4}$ & 1.88 \\
\hline $20 \times 20 \times 20$ & $2.65 \times 10^{-5}$ & 1.94 \\
\hline $40 \times 40 \times 40$ & $6.56 \times 10^{-6}$ & 1.97 \\
\hline $80 \times 80 \times 80$ & $1.63 \times 10^{-6}$ & 1.99 \\
\hline $160 \times 160 \times 160$ & $4.06 \times 10^{-7}$ & \\
\hline
\end{tabular}

Table 7.1: $L_{2}$ norm of the error and order of accuracy for the displacement (unknown $u$ ).

\begin{tabular}{|c|c|c|}
\hline Cells & $\|$ Error $\|_{2}$ & Order \\
\hline $10 \times 10 \times 10$ & $3.56 \times 10^{-3}$ & 1.87 \\
\hline $20 \times 20 \times 20$ & $8.80 \times 10^{-4}$ & 1.94 \\
\hline $40 \times 40 \times 40$ & $2.18 \times 10^{-4}$ & 1.97 \\
\hline $80 \times 80 \times 80$ & $5.42 \times 10^{-5}$ & 1.98 \\
\hline $160 \times 160 \times 160$ & $1.35 \times 10^{-5}$ & \\
\hline
\end{tabular}

Table 7.2: $L_{2}$ norm of the error and order of accuracy for the velocity (unknown $v$ ).

and compute the $L_{2}$ norm of the error for each case, obtaining numerically the order of accuracy of the scheme. Table 7.1 illustrates the error and order of accuracy for the unknown $u$ (particle displacement) for all the meshes considered, whereas table 7.2 shows the error and the order of accuracy for the unknown $v$ (particle velocity) for the different meshes. The output time is $t_{\max }=0.1$ while the size of the time step used verifies the stability constraints. Other values of the parameters of the model taken here are $\zeta=65.4$, $\eta=0.0147$ and $\nu=10$. The results obtained reveal that the theoretical second order of accuracy is attained in practice.

\subsection{Von Neumann Stability Analysis}

In this section, we conduct a stability analysis of the numerical scheme used in the present work, that is FV-RK2-TVD, applied to the 3D diffusive-viscous-wave (3D-DVW) model. A very useful technique to analyze the stability of a numerical scheme is the so called von Neumann's method, which is of particular interest in the context of equations, or systems of equations, with constant coefficients, like the one we are dealing with in this work. There are many references in which this method is described such as Durran (2013), applied to wave equations and geophysical problems; Godlewski and Raviart (1996), LeVeque (2002), Toro (2013) or Toro et al. (2009) mainly focused on hyper- 
bolic problems. Moreover, in Regan (2002) there is an interesting application of the von Neumann's technique to symplectic integration methods in the context of Hamiltonian equations based on systems of ODEs.

We start by writing the DVW model as

$$
\left(\begin{array}{l}
u_{t} \\
v_{t}
\end{array}\right)=\left(\begin{array}{cc}
0 & 1 \\
\nu^{2} \Delta & \eta \Delta-\zeta
\end{array}\right)\left(\begin{array}{l}
u \\
v
\end{array}\right)
$$

where $u_{t}=\frac{\partial u}{\partial t}$ and $v_{t}=\frac{\partial v}{\partial t}$ and we have used the differential operator $\Delta$. The proposed numerical scheme, based on a FV technique with RK2-TVD, requires, at each time step, reconstructing fluxes and values, where needed, using piecewise reconstruction from cell averages. We have used here piecewise linear reconstruction. The evolution step is usually carried out via an appropriate ODEs solver. Since we are using second order spatial reconstruction we resort to a second order ODEs solver, such as the RK2-TVD scheme. This method verifies the Total Variation Diminishing (TVD) property, which is especially efficient to achieve solutions free of non-physical oscillations.

The RK2-TVD method is constructed in two stages. The first stage, written in matrix form, reads as

$$
\left(\begin{array}{c}
u_{i, j, k}^{(1)} \\
v_{i, j, k}^{(1)}
\end{array}\right)=\left(\begin{array}{c}
u_{i, j, k}^{n} \\
v_{i, j, k}^{n}
\end{array}\right)+\delta t\left(\begin{array}{cc}
0 & 1 \\
\nu^{2} \Delta & \eta \Delta-\zeta
\end{array}\right)\left(\begin{array}{c}
u_{i, j, k}^{n} \\
v_{i, j, k}^{n}
\end{array}\right) .
$$

If we introduce now in Eq.(7.12) the approximation of the laplacians given by

$$
\begin{aligned}
& \Delta u_{i, j, k}^{n} \approx \frac{1}{\Delta x^{2}}\left(u_{i+1, j, k}^{n}-2 u_{i, j, k}^{n}+u_{i-1, j, k}^{n}\right)+ \\
& \frac{1}{\Delta y^{2}}\left(u_{i, j+1, k}^{n}-2 u_{i, j, k}^{n}+u_{i, j-1, k}^{n}\right)+ \\
& \frac{1}{\Delta z^{2}}\left(u_{i, j, k+1}^{n}-2 u_{i, j, k}^{n}+u_{i, j, k-1}^{n}\right)
\end{aligned}
$$

and consider the simplified case in which $\Delta x=\Delta y=\Delta z=h$ we get the following expression

$$
\left(\begin{array}{c}
u_{i, j, k}^{(1)} \\
v_{i, j, k}^{(1)}
\end{array}\right)=\left(\begin{array}{c}
u_{i, j, k}^{n} \\
v_{i, j, k}^{n}
\end{array}\right)+\left(\begin{array}{c}
\delta t v_{i, j, k}^{n} \\
-\beta v_{i, j, k}^{n}+P+Q
\end{array}\right)
$$


with

$$
\begin{gathered}
P=\alpha\left(v_{i+1, j, k}^{n}+v_{i-1, j, k}^{n}+v_{i, j+1, k}^{n}+v_{i, j-1, k}^{n}+v_{i, j, k+1}^{n}+v_{i, j, k-1}^{n}-6 v_{i, j, k}^{n}\right) \\
Q=\gamma\left(u_{i+1, j, k}^{n}+v_{i-1, j, k}^{n}+u_{i, j+1, k}^{n}+u_{i, j-1, k}^{n}+v_{i, j, k+1}^{n}+u_{i, j, k-1}^{n}-6 u_{i, j, k}^{n}\right),
\end{gathered}
$$

and we have introduced the notation $\beta=\delta t \zeta, \gamma=\frac{\nu^{2} \delta t}{h^{2}}$ and $\alpha=\frac{\eta \delta t}{h}$, $\delta t$ being the size of the time step. We remark that the spatial reconstruction has already been performed and we are using cell averages of the functions $u$ and $v$.

In this first stage the values $u_{i, j, k}^{(1)}$ and $v_{i, j, k}^{(1)}$ are computed.

\section{The second stage reads}

$$
\left(\begin{array}{c}
u_{i, j, k}^{n+1} \\
v_{i, j, k}^{n+1}
\end{array}\right)=\frac{1}{2}\left[\left(\begin{array}{c}
u_{i, j, k}^{n} \\
v_{i, j, k}^{n}
\end{array}\right)+\left(\begin{array}{c}
u_{i, j, k}^{(1)} \\
v_{i, j, k}^{(1)}
\end{array}\right)+\delta t\left(\begin{array}{cc}
0 & 1 \\
\nu^{2} \Delta & \eta \Delta-\zeta
\end{array}\right)\left(\begin{array}{c}
u_{i, j, k}^{(1)} \\
v_{i, j, k}^{(1)}
\end{array}\right)\right] .
$$

After some algebra we obtain the updated values

$$
\left(\begin{array}{c}
u_{i, j, k}^{n+1} \\
v_{i, j, k}^{n+1}
\end{array}\right)=\left(\begin{array}{c}
\delta t\left(1-\frac{\beta}{2}-3 \alpha\right) v_{i, j, k}^{n}+(1-3 \delta t \gamma) u_{i, j, k}^{n}+\frac{\delta t}{2}\left(\alpha \tilde{V}_{1}+\gamma \tilde{U}_{1}\right) \\
c_{1} v_{i, j, k}^{n}+c_{2} u_{i, j, k}^{n}+c_{3} \tilde{V}_{1}+c_{4} \tilde{U}_{1}+\frac{\alpha^{2}}{2}\left(\tilde{V}_{2}+2 \tilde{V}_{3}\right)+\frac{\alpha \gamma}{2}\left(\tilde{U}_{2}+2 \tilde{U}_{3}\right)
\end{array}\right),
$$

where

$$
\begin{aligned}
\tilde{V}_{1}= & v_{i+1, j, k}^{n}+v_{i-1, j, k}^{n}+v_{i, j+1, k}^{n}+v_{i, j-1, k}^{n}+v_{i, j, k+1}^{n}+v_{i, j, k-1}^{n}, \\
\tilde{U}_{1}= & u_{i+1, j, k}^{n}+u_{i-1, j, k}^{n}+u_{i, j+1, k}^{n}+u_{i, j-1, k}^{n}+u_{i, j, k+1}^{n}+u_{i, j, k-1}^{n}, \\
\tilde{V}_{2}= & v_{i+2, j, k}^{n}+v_{i-2, j, k}^{n}+v_{i, j+2, k}^{n}+v_{i, j-2, k}^{n}+v_{i, j, k+2}^{n}+v_{i, j, k-2}^{n}, \\
\tilde{U}_{2}= & u_{i+2, j, k}^{n}+u_{i-2, j, k}^{n}+u_{i, j+2, k}^{n}+u_{i, j-2, k}^{n}+u_{i, j, k+2}^{n}+u_{i, j, k-2}^{n}, \\
\tilde{V}_{3}= & v_{i+1, j+1, k}^{n}+v_{i+1, j-1, k}^{n}+v_{i-1, j+1, k}^{n}+v_{i-1, j-1, k}^{n}+v_{i, j+1, k+1}^{n}+ \\
& v_{i, j+1, k-1}^{n}+v_{i, j-1, k+1}^{n}+v_{i, j-1, k-1}^{n}, \\
\tilde{U}_{3}= & u_{i+1, j+1, k}^{n}+u_{i+1, j-1, k}^{n}+u_{i-1, j+1, k}^{n}+u_{i-1, j-1, k}^{n}+u_{i, j+1, k+1}^{n}+ \\
& u_{i, j+1, k-1}^{n}+u_{i, j-1, k+1}^{n}+u_{i, j-1, k-1}^{n},
\end{aligned}
$$


and the coefficients are

$$
\begin{aligned}
& c_{1}=1-\beta+3 \alpha(\beta+7 \alpha-2)-3 \gamma \delta t, \\
& c_{2}=3 \gamma(7 \alpha-2) \\
& c_{3}=\alpha\left(1-6 \alpha-\frac{\beta}{2}\right)+\frac{\gamma \delta t}{2} \\
& c_{4}=\gamma(1-6 \alpha) .
\end{aligned}
$$

In order to apply von Neumann's technique we establish the ansatz

$$
\begin{array}{ll}
u_{i \pm 1, j, k}^{n}=e^{ \pm I i \theta} u_{i, j, k}^{n} ; & v_{i \pm 1, j, k}^{n}=e^{ \pm I i \theta} v_{i, j, k}^{n}, \\
u_{i, j \pm 1, k}^{n}=e^{ \pm I j \phi} u_{i, j, k}^{n} ; & v_{i, j \pm 1, k}^{n}=e^{ \pm I j \phi} v_{i, j, k}^{n}, \\
u_{i, j, k \pm 1}^{n}=e^{ \pm I k \psi} u_{i, j, k}^{n} ; & v_{i, j, k \pm 1}^{n}=e^{ \pm I k \psi} v_{i, j, k}^{n}, \\
u_{i \pm 2, j, k}^{n}=e^{ \pm 2 I i \theta} u_{i, j, k}^{n} ; & v_{i \pm 2, j, k}^{n}=e^{ \pm 2 I i \theta} v_{i, j, k}^{n}, \\
u_{i, j \pm 2, k}^{n}=e^{ \pm 2 I j \phi} u_{i, j, k}^{n} ; & v_{i, j \pm 2, k}^{n}=e^{ \pm 2 I j \phi} v_{i, j, k}^{n}, \\
u_{i, j, k \pm 2}^{n}=e^{ \pm 2 I k \psi} u_{i, j, k}^{n} ; & v_{i, j, k \pm 2}^{n}=e^{ \pm 2 I k \psi} v_{i, j, k}^{n},
\end{array}
$$

where $I=\sqrt{-1}$ is the imaginary unit and $\theta, \phi, \psi$ are the phase angles. We introduce the expressions (7.20) in (7.17-7.19) to yield

$$
\left(\begin{array}{c}
u_{i, j, k}^{n+1} \\
v_{i, j, k}^{n+1}
\end{array}\right)=\mathbf{A}\left(\begin{array}{c}
u_{i, j, k}^{n} \\
v_{i, j, k}^{n}
\end{array}\right)
$$

where $\mathbf{A}=\left(\begin{array}{cc}A_{1,1} & A_{1,2} \\ A_{2,1} & A_{2,2}\end{array}\right)$ is the amplification matrix.

In order to get a stable solution a necessary condition is $|\rho(\mathbf{A})| \leq 1$, see for instance Godlewski and Raviart (1996) or Durran (2013), where $\rho(\mathbf{A})$ is the spectral radius of the matrix A. As an alternative, some matrix norm could also be used. When obtaining the 
elements of $\mathbf{A}$ it is useful to apply trigonometric relations, such as

$$
\begin{aligned}
& e^{a}=\cos a+I \sin a \\
& \cos a=\frac{e^{I a}+e^{-I a}}{2} \\
& \cos a=1-2 \sin ^{2} a \\
& \cos (a \pm b)=\cos a \cos b \mp \sin a \sin b .
\end{aligned}
$$

Using the above relations and with the aid of algebraic manipulators we can finally obtain the elements of the matrix $\mathbf{A}$, which read

$$
\begin{aligned}
& A_{1,1}=1-2 \omega \mu \\
& A_{1,2}=\frac{\delta t}{2}(2-\beta-4 \omega \alpha) \\
& A_{2,1}=-\gamma(-\alpha(3-2 \xi)+(-2+12 \alpha)(3-2 \omega)-4 \alpha \kappa-21 \alpha+6) \\
& A_{2,2}=1+\left(\mu+2 \alpha-\beta \alpha-12 \alpha^{2}\right)(3-2 \omega)+\alpha^{2}(3-2 \xi)+4 \alpha^{2} \kappa+\sigma,
\end{aligned}
$$

where $\omega=\sin ^{2} \frac{\theta}{2}+\sin ^{2} \frac{\phi}{2}+\sin ^{2} \frac{\psi}{2}, \xi=\sin ^{2} \theta+\sin ^{2} \phi+\sin ^{2} \psi, \kappa=\cos \theta \cos \psi+$ $\cos \theta \cos \phi+\cos \phi \cos \psi, \sigma=-\beta-6 \alpha-3 \mu+3 \alpha \beta+21 \alpha^{2}$ and $\mu=\gamma \delta t=\frac{\nu^{2} \delta t^{2}}{h^{2}}$.

Let us recall that, in order to analyze the stability, we need to compute the spectral radius of the matrix A. The eigenvalues of this matrix are obtained as

$$
\begin{aligned}
& \lambda_{1}=\frac{\left(A_{1,1}+A_{2,2}\right)+\sqrt{\left(A_{1,1}+A_{2,2}\right)^{2}-4\left(A_{1,1} A_{2,2}-A_{1,2} A_{2,1}\right)}}{2} \\
& \lambda_{2}=\frac{\left(A_{1,1}+A_{2,2}\right)-\sqrt{\left(A_{1,1}+A_{2,2}\right)^{2}-4\left(A_{1,1} A_{2,2}-A_{1,2} A_{2,1}\right)}}{2} .
\end{aligned}
$$

The spectral radius of the matrix is calculated according to $\rho(\mathbf{A})=\max \left(\left|\lambda_{1}\right|,\left|\lambda_{2}\right|\right)$. We note that if the discriminant: $d=\left(A_{1,1}+A_{2,2}\right)^{2}-4\left(A_{1,1} A_{2,2}-A_{1,2} A_{2,1}\right)<0$, the eigenvalues are complex and in that case the modulus of both eigenvalues is the same, reading in this case $\left|\lambda_{1}\right|=\left|\lambda_{2}\right|=\sqrt{\Re^{2}+\Im^{2}}$ where $\Re=\frac{A_{1,1}+A_{2,2}}{2}$ and $\Im=\frac{\sqrt{-d}}{2}$.

We display in plots $\mu-\alpha$ the regions where the spectral radius is less or equal one (white zone). The results are shown in figure 7.6. We notice that the full white region is not valid for stability since, as stated before, the condition based on the spectral radius is necessary, 
but not sufficient to guarantee stability of the numerical scheme, see for instance Durran (2013) or Sousa (2003) for more details on necessary and sufficient conditions in von Neumann analysis. Due to this fact, we identify in the plots the zones where stability is assured. Furthermore, in the stability region, the value of the amplification factor, which is equivalent to the spectral radius, is equal, or very close to 1.0 , which is an interesting feature in terms of accuracy.

With the aim of verifying the stability region previously obtained, we consider the following problem

$$
\left\{\begin{array}{l}
\frac{\partial v}{\partial t}=-100 v+10 \Delta v+10 \Delta u, \forall(x, y, z) \in(-10,10), \forall t>0 \\
\frac{\partial u}{\partial t}=v, \forall(x, y, z) \in(-10,10), \forall t>0 \\
\left.\frac{\partial u}{\partial \mathbf{n}}\right|_{\Gamma}=\left.\frac{\partial v}{\partial \mathbf{n}}\right|_{\Gamma}=0, \forall t>0 \\
u(x, y, z, 0)=\left\{\begin{array}{cc}
10.0 & \text { if } 3.0 \leq(x, y, z) \leq 7.0 \\
0.1 & \text { otherwise }
\end{array}\right. \\
v(x, y, z, 0)=\left\{\begin{array}{cc}
5.0 & \text { if } 3.0 \leq(x, y, z) \leq 7.0 \\
0.1 & \text { otherwise }
\end{array}\right.
\end{array}\right.
$$

We solve the problem equation (7.25) inside the stability region, using the values $\alpha=0.1$, $\mu=0.1$ and $\beta=1.5$. The solution achieved is displayed in figure 7.7 for an output time $t_{\max }=60.0$. The mesh used has $30 \times 30 \times 30$ cells.

\subsection{Numerical Results}

We study a $[0,1000] \times[0,1000] \times[0,1000] \mathrm{m}, 3$-D fluid-saturated model, where we have generated a finite volume cartesian regular mesh with $500 \times 500 \times 50$ control volumes, with an $80 \mathrm{~m}$ thick oil-, water- and gas-saturated layer ingrained between two dry sandstone layers. The water-saturated layer, $300 \mathrm{~m}$ in length, is located at the centre of the fluid-saturated layer, as shown in figure 7.2 above, with the other two (oil and gas) layers 

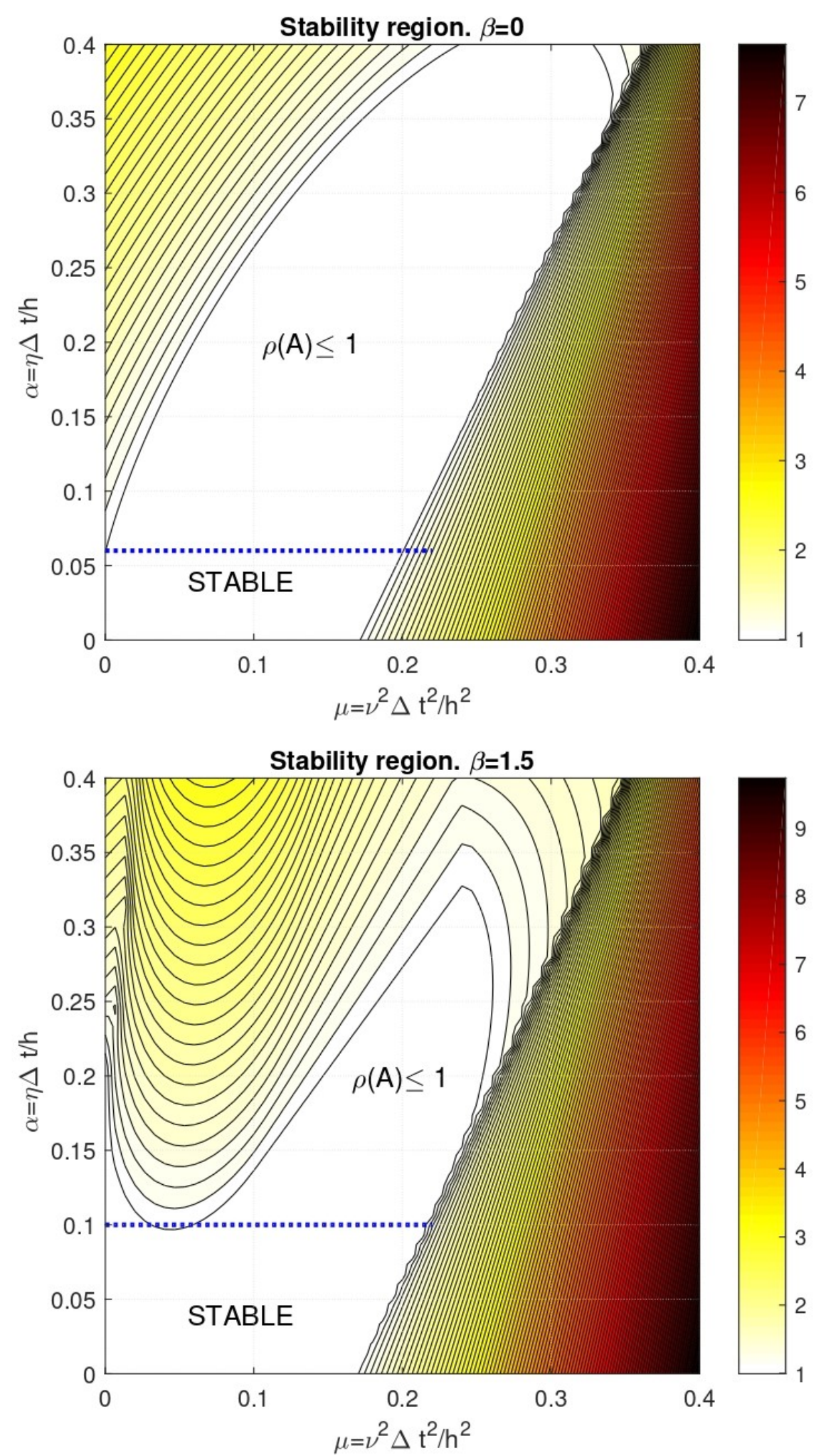

Figure 7.6: Region in the plane $\mu-\alpha$ where the spectral radius satisfy $\rho(\mathbf{A}) \leq 1$. The stability region is also identified. The results have been obtained using $\beta=0$. (top frame) and $\beta=1.5$ (bottom frame). 




Figure 7.7: Numerical solution of the problem Eq.(7.25) using $\alpha=0.1, \mu=0.1, \beta=1.5$, output time $t_{\max }=60.0$. The plot is a cut with the plane $y=5.0$. 
having lengths of $350 \mathrm{~m}$ each. The physical parameters applied to the model are similar to that used by (Mensah et al., 2019) and are presented in Table 5.2. The source involved is a Ricker wavelet with an amplitude spectrum peak of $30 \mathrm{~Hz}$ (see Figure 2.1 for an illustration of a Ricker wavelet with a peak amplitude spectrum of $30 \mathrm{~Hz}$, and its corresponding frequency spectrum), which is integrated over the time step and spread over the cells (Mensah et al., 2019). Empirical studies have, in most cases, proved that the Ricker wavelet approximates the seismic spectra better than other families of approximating functions and it at the same time requires fewer parameters compared to other possible approximations. It is for these reasons that we use the Ricker wavelet. The source is located at the centre of the surface (refer to figure 7.2). The source delay is $40 \mathrm{~ms}$, and the step-sizes are $2.0 \mathrm{~m}$ for all directions. The von Neumann Stability Criteria that control the time step for the diffusive-viscous wave equation discretised with the finite volume method is given in Section 7.3. The total time for the simulation is $1400 \mathrm{~ms}$. Three receivers are placed at the surface (refer to fig. 7.2). We present results for both particle motions (velocity and displacement) since a system of equation has been developed, that is, velocity-displacement formulations.

We present snapshots for particle displacements and particle velocities in figures 7.8 and 7.9 , respectively, at randomly chosen times for both the diffusive-viscous waves and the visco-acoustic waves. Seismograms from receivers A, B, C (refer to figure 7.2) are produced and comparisons are made between those generated for both the visco-acoustic and diffusive-viscous waves. It is worth noting that receiver A records the information from the oil-saturated region, receiver B also records for the water-saturated part and receiver $\mathrm{C}$ records for the gas-saturated area. Multiple reflections generated from the layer are as well indicated on the seismograms. From figure 7.10, multiples from the water-, oil- and gas-saturated layers are visible in the case of the visco-acoustic waves, but they are nearly unseen in the case of the diffusive-viscous waves.

In comparison with seismograms from the visco-acoustic case, seismograms from the diffusive-viscous case show an apparent amplitude attenuation that appears distinctly within the model but most importantly, within the fluid-saturated layer. The relative time locations for particle velocity and particle displacements for both the visco-acoustic and 


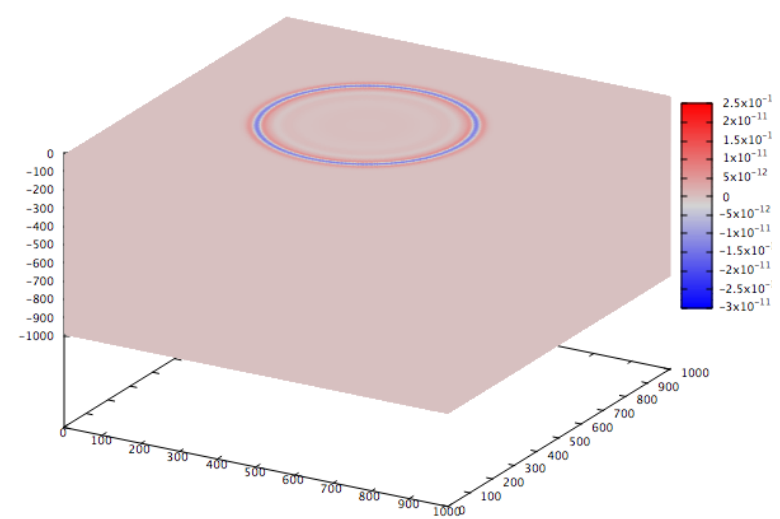

(a) $250 \mathrm{~ms}$

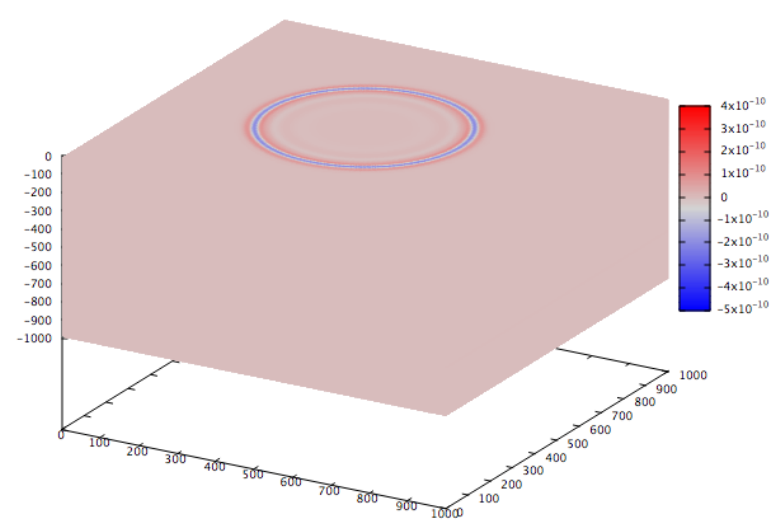

(c) $250 \mathrm{~ms}$

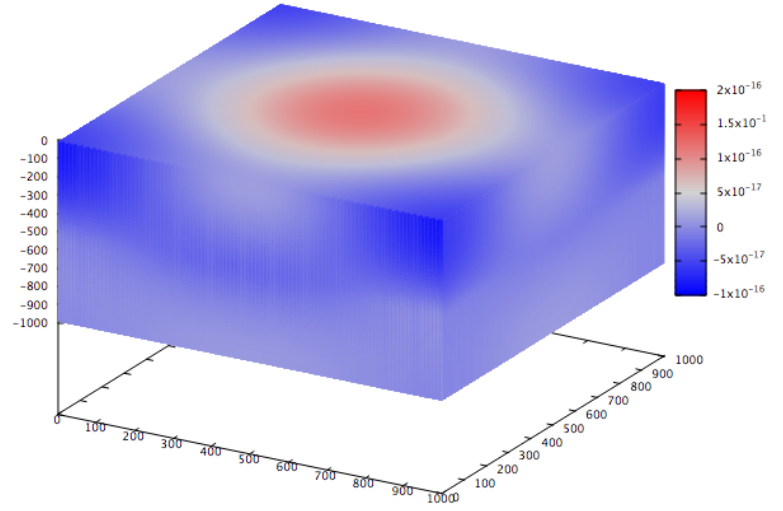

(b) $1400 \mathrm{~ms}$

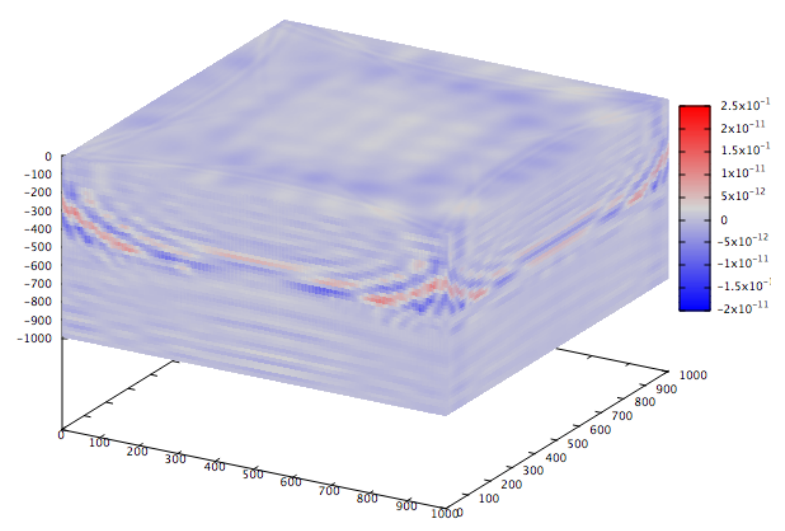

(d) $1400 \mathrm{~ms}$

Figure 7.8: Snapshots of diffusive-viscous wave-fields (a), (b) and visco-acoustic wavefields (c), (d) in a 3-D fluid-saturated medium for Particle Displacement (notice varying color scaling) 


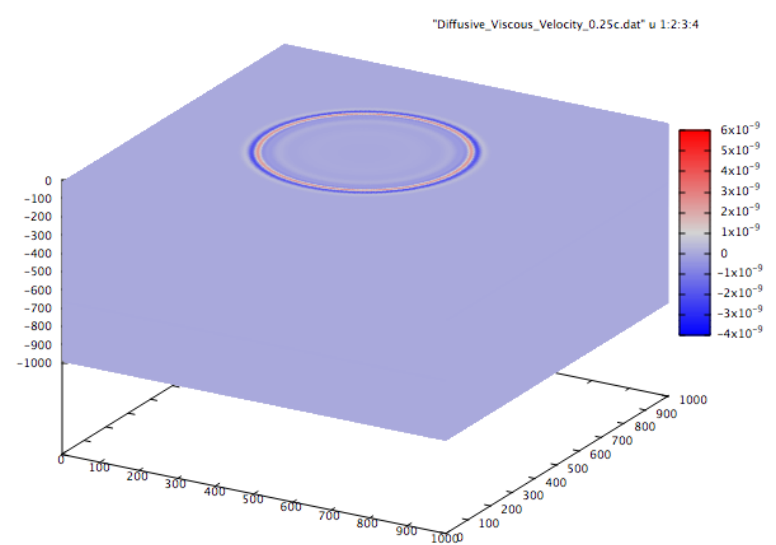

(a) $250 \mathrm{~ms}$



(c) $250 \mathrm{~ms}$



(b) $1400 \mathrm{~ms}$

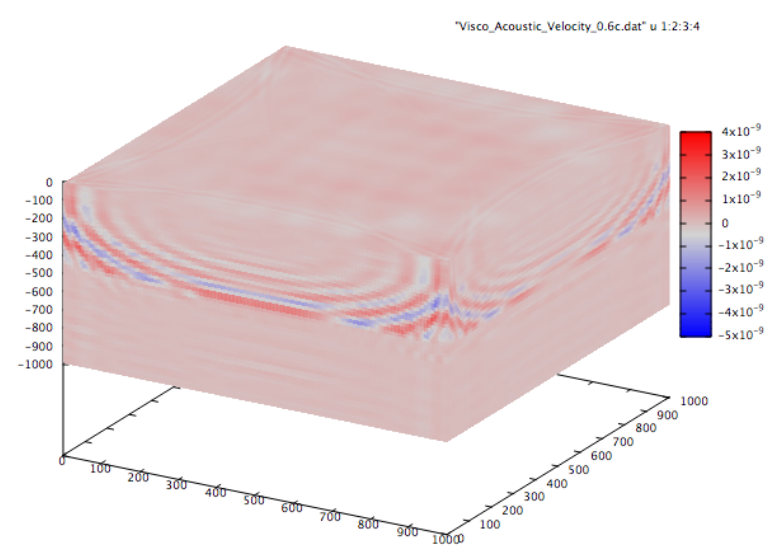

(d) $1400 \mathrm{~ms}$

Figure 7.9: Snapshots of diffusive-viscous wave-fields (a), (b) and visco-acoustic wavefields (c), (d) in a 3-D fluid-saturated medium for Particle Velocity (notice varying color scaling) 
diffusive-viscous scenarios of the amplitude attenuation of receiver $\mathrm{A}$ is about $0.4 \mathrm{~s}$, with receiver B taking place at about $0.02 \mathrm{~s}$, and finally, receiver $\mathrm{C}$ occurring at about $0.4 \mathrm{~s}$ (refer to figure 7.10). From the numerical results, the same snapshots are produced for both the visco-acoustic and diffusive-viscous cases at $250 \mathrm{~ms}$ after the source has propagated from the source location within the first dry sandstone layer. It is evident from figures 7.8 and 7.9 that there is a significant attenuation as the diffusive and viscous waves propagate through the fluid-saturated layer for both displacement and velocity results. The gas-saturated layer presents a lower attenuating effect when compared to both the oil- and water-saturated media which is evident on seismograms. The low-attenuating effects of the gas-saturated layer can be associated with the low values of the diffusive attenuation parameter, $\zeta$, as compared to oil- and water-saturated layers. The layer with higher attenuating parameters, that is the water-saturated layer, present a stronger attenuating effect. It can thus be concluded that the attenuation parameters significantly affect numerical results. These parameters that can be obtained from laboratory analysis or through seismic inversion from seismic field data rely primarily upon permeability, stiffness, porosity and other fluid-saturated rock parameters (Goloshubin \& Korneev, 2005; V. Korneev, 2007; V. A. Korneev et al., 2004; Mensah et al., 2019).

\subsection{Discussion}

We attempt to describe the frequency-dependent seismic reflections from a fluid-saturated model numerically. The method can be expected to evaluate the fluid-related effects likely produced by fluid flow in the pores of reservoirs according to the diffusive-viscous theory. Nevertheless, assuming that the wave propagates in vertical or nearly vertical directions in the media with lateral homogeneity, and neglecting the S-wave, the diffusive-viscous wave equation can be considered as an extended acoustic wave equation rather than an elastic one, and it can numerically model the reflections of both $\mathrm{P}$ and $\mathrm{S}$ waves or even the converted wave. Due to the anelastic properties of the media through which the waves propagate, the propagating waves will decay. Goloshubin and Korneev (2000, 2005); Goloshubin et al. (2002); V. A. Korneev et al. (2004); V. Korneev (2007) studied and ob- 


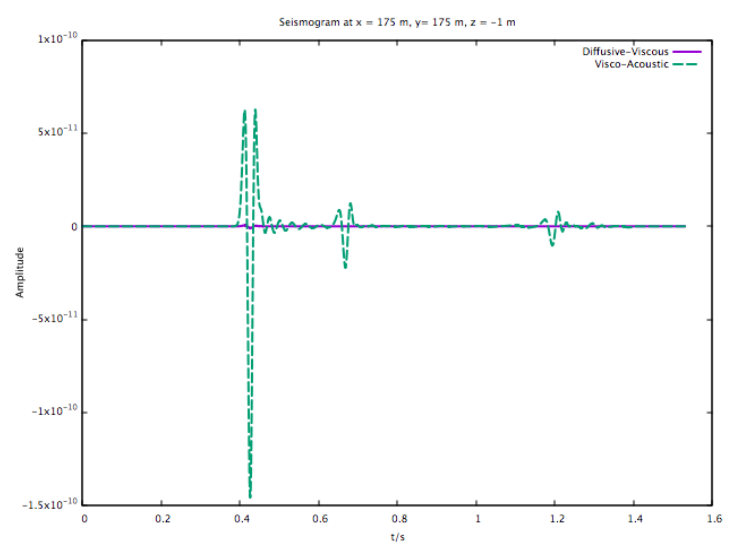

(a) Receiver A for Particle Displacement

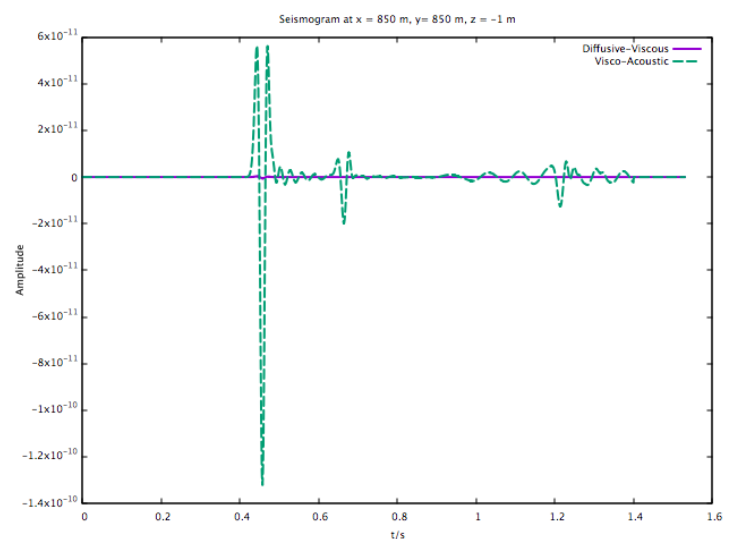

(c) Receiver C for Particle Displacement

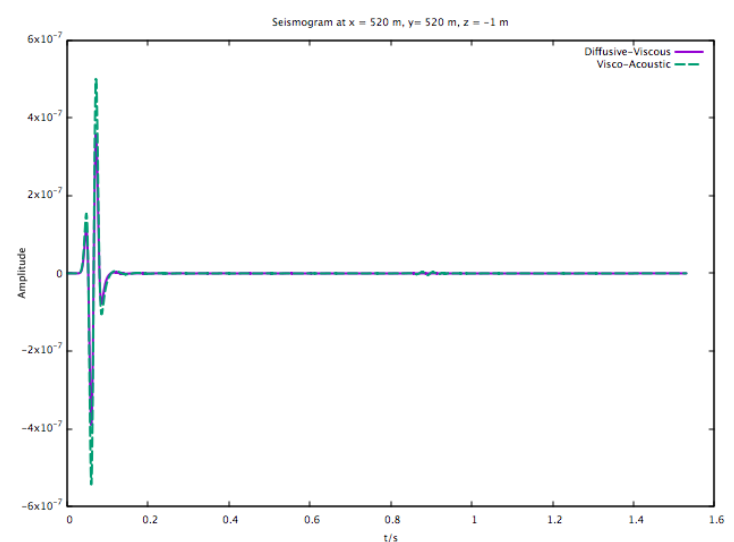

(e) Receiver B for Particle Velocity

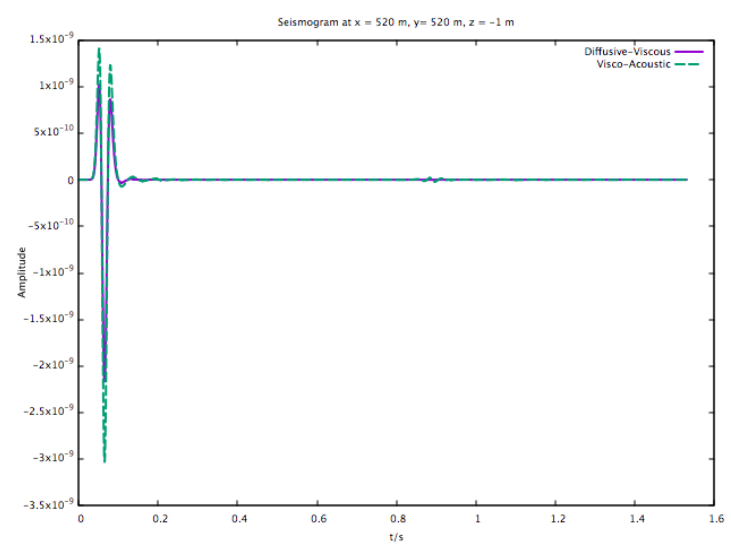

(b) Receiver B for Particle Displacement

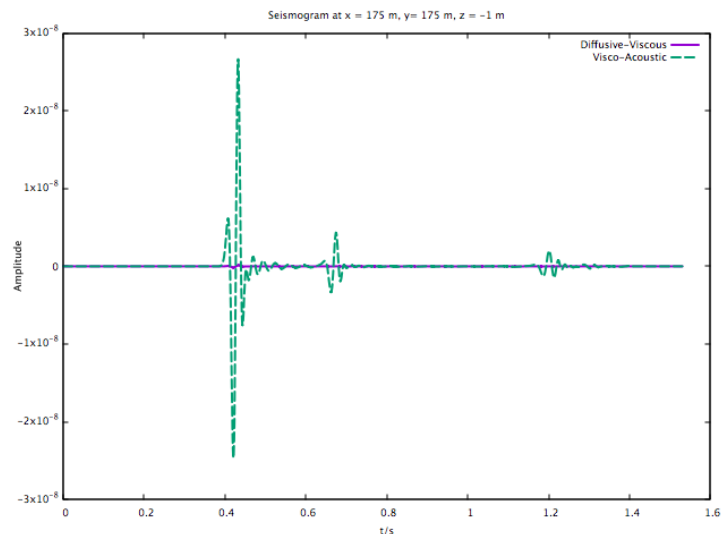

(d) Receiver A for Particle Velocity

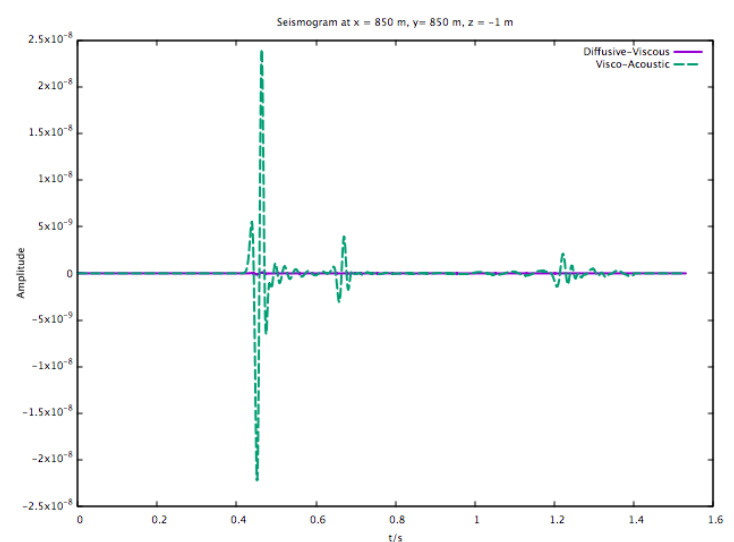

(f) Receiver C for Particle Velocity

Figure 7.10: Seismograms generated from Particle Displacement and Particle Velocity for diffusive-viscous wave (solid line) compared with visco-acoustic wave (dashed-line) for three receivers (A, B and C) placed on the surface of the model with multiple reflections indicated 
tained the diffusive attenuation parameters, $(\zeta)$ and viscous attenuation parameters, $(\eta)$ applied in this paper from laboratory experiments. The parameters mentioned above which undeniably have a significant effect on snapshots and seismograms, can be acquired analytically from the fundamental laws of physics or, in practice, through Amplitude Versus Offset/Angle (AVO/AVA) analysis. Notably, the low-frequency phenomenon related to hydrocarbon accumulations emerges from a range of effects associated with the different physical properties and mechanisms of reservoirs. It is rather strenuous (if not impossible) to describe the seismic low-frequency anomalies by simultaneously taking into consideration all the possible related factors in the reservoir (Goloshubin \& Korneev, 2000, 2005; Goloshubin et al., 2002; V. A. Korneev et al., 2004; V. Korneev, 2007).

The main aim of our numerical method is to develop a finite-volume scheme to understand the wave propagation in an almost realistic three-dimensional fluid-saturated media based on the diffusive and viscous effects of the pore fluid in reservoirs. The reason for implementing FV methods is due to its versatility and direct approach when dealing with irregular grids, and also FV methods ensure the conservation of the variables involved. FV methods often do a better job when dealing with source terms than FD methods. The numerical results generated with this FV scheme can be used to interpret field data and therefore contribute to seismic imaging and seismic inversion.

\subsection{Conclusion}

We have developed a three-dimensional finite volume scheme for modelling the seismic wave propagation based on the diffusive-viscous theory. The DVWE-based simulation presented in this thesis displays waves propagating in a fluid-saturated medium using FVM on a Cartesian grid. To our knowledge, it is the first time the FVM is applied to the DVWE in three dimensions.

Snapshots and seismograms, when compared with the visco-acoustic case, present a significant attenuation of the diffusive-viscous waves as they travel through the fluidsaturated layer. The gas-saturated medium shows a lower attenuating effect when compared to the oil- and water-saturated media and therefore, it can be said that the diffusive 
attenuation parameter $\zeta$ has a remarkable impact on the numerical results. Our finite volume scheme has exhibited its flexibility and proves to be stable even for a time step larger than that given in Zhao, Gao, and Zhao (2014) for the finite difference scheme. This FV scheme significantly serves as an improvement upon the scheme previously developed, and the DVWE simulations based on this FV scheme can be, adopted as a guide in frequency-dependent fluid delineation methods. That being said, the numerical scheme applied to the modelling of seismic wave propagation should be much considered to obtain optimum results. Besides, explicitly including diffusive and viscous attenuation terms in seismic wave numerical modelling is fundamental for realistic seismic imaging and data interpretation. The three-dimensional technique will permit to study the wave field due to laterally heterogeneous structures.

\subsection{Summary}

In this Chapter, we have applied the second-order Runge-Kutta FVM to solve the threedimensional DVWE (equation 2.3) in a three-dimensional Cartesian coordinate system $(x, y, z)$. We have also applied the Mur Absorbing Boundary Condition (Clayton \& Engquist, 1977), which are of absorbing type, on all sides of the model. The elevations $(z)$ have been assumed to be increasing below mean sea level, that is, negatively downwards as in a real case. The grid spacing is equal in all directions, that is, $x-, y-$ and $z$-directions, and the time step is, regulated by the stability criteria. 


\section{Chapter 8}

\section{Conclusions and final remarks}

\subsection{Summary}

The objectives of this thesis have been to develop an accurate and stable numerical model for the diffusive-viscous wave equation over a Cartesian domain with a boundary condition of absorbing type while maintaining computational efficiency. What we have presented in this thesis using time-domain finite volume method, has to some extent achieved these objectives.

As a concise summary of this thesis, in Chapter two we commenced with comprehensive PDE models of the diffusive-viscous wave equation, in Chapter three we presented the discretization of those equations along with other methodologies for the scheme which can be implemented on unstructured grids as well. In Chapter four, we discussed the considerations for simulating the scheme in two dimensions and delved deeper into the methodologies. In Chapter five, the diffusive-viscous wave equation was modelled in two-dimensions on a simple Cartesian mesh, with the goal of tackling the accuracy of the numerical scheme. In Chapter six, we presented a three-dimensional consideration of the scheme which extended the methods in Chapter four. In Chapter seven, we used the finite volume method to model the diffusive-viscous wave equation on a three-dimensional Cartesian mesh with the aim of tackling the accuracy of the numerical scheme using the von Neumann stability analysis.

In the numerical examples in Chapters five and seven, we presented a two-dimensional 
and three-dimensional finite volume scheme for modelling the low-frequency effects based on the diffusive-viscous theory. The DVWE-based simulation presented in this thesis not only displayed waves propagating in a fluid-saturated medium using FVM on a Cartesian grid, but it has also delineated the frequency-dependent effects. To our knowledge, it is the first time the FVM is applied to the DVWE in both two and three dimensions. Snapshots and seismograms, when compared with the acoustic and visco-acoustic cases, have presented a significant attenuation of the diffusive-viscous waves as they propagated through the fluid-saturated layer. The gas-saturated medium have depicted a lower attenuating effect when compared to the oil- and water-saturated media and therefore, it can be said that the diffusive attenuation parameter $\zeta$ had a remarkable impact on the numerical results. The finite volume scheme presented in this thesis exhibits its flexibility and proves to be stable even for a time step larger than that given in (Zhao, Gao, \& Zhao, 2014) for the finite difference scheme. This FV scheme significantly serves as an improvement upon the scheme previously developed, and the DVWE simulations based on this FV scheme can be adopted as a guide in frequency-dependent fluid delineation methods. That being said, the numerical scheme applied to the modelling of seismic wave propagation should be much considered to obtain optimum results. Besides, explicitly including diffusive and viscous attenuation terms in seismic wave numerical modelling is fundamental for realistic seismic imaging and data interpretation. The two and three-dimensional techniques will allow for the study of wavefield due to laterally heterogeneous structures.

\subsection{Recommendations for future research}

Based on the results of this research, the subsequent recommendations are outlined for further research in this field:

- Implement a higher order time integration method so as to improve upon the accuracy of the already existing scheme in time and space as well. Caution must be exercised so that the spatial discretization, if maintained, will not be nullified by an inaccurate time-stepping scheme since the DVWE is an equation of special kind.

- Implement a higher order spatial discretization using the piecewise parabolic re- 
construction or higher flux reconstruction methods such as the WENO-family of reconstruction.

- Apply parametric statistics to attempt to extract the characteristic parameters of the anisotropy heterogeneity which can be generated using a stochastic method such as the Von Karmán method for the characterisation of random velocity fields, and based on the random velocity fields generated, deduce random fields as well for the diffusive and viscous parameters.

- Apply the numerical schemes generated in this research on real observations in order to evaluate its performance. 


\section{References}

Aagaard, B. T., Hall, J. F., \& Heaton, T. H. (2001). Characterization of near-source ground motions with earthquake simulations. Earthquake Spectra, 17(2), 177-207.

Agreste, S., \& Ricciardello, A. (2011). Simulation of seismic wave propagation in 3d heterogeneous media: a parallel computing approach. Communications in Applied and Industrial Mathematics, 2(1).

Aguirre, M., Gil, A. J., Bonet, J., \& Carreño, A. A. (2014). A vertex centred finite volume jameson-schmidt-turkel (jst) algorithm for a mixed conservation formulation in solid dynamics. Journal of Computational Physics, 259, 672-699.

Aguirre, M., Gil, A. J., Bonet, J., \& Lee, C. H. (2015). An upwind vertex centred finite volume solver for lagrangian solid dynamics. Journal of Computational Physics, $300,387-422$.

A. Jayantha, P., IW Turner. (2001). A comparison of gradient approximations for use in finite-volume computational models for two-dimensional diffusion equations. $\mathrm{Nu}$ merical Heat Transfer, Part B: Fundamentals, 40(5), 367-390.

Al-Athel, K., \& Gadala, M. (2011). Eulerian volume of solid (vos) approach in solid mechanics and metal forming. Computer methods in applied mechanics and engineering, 200(25-28), 2145-2159.

Allen, J. B., \& Berkley, D. A. (1979). Image method for efficiently simulating small-room acoustics. The Journal of the Acoustical Society of America, 65(4), 943-950.

Alterman, Z., \& Karal Jr, F. (1968). Propagation of elastic waves in layered media by finite difference methods. Bulletin of the Seismological Society of America, 58(1), $367-398$.

Anné, L., Tran, Q. H., \& Symes, W. W. (1997). Dispersion and cost analysis of some finite difference schemes in one-parameter acoustic wave modeling. Computational Geosciences, 1(1), 1-33. 
Aretz, M. (2012). Combined wave and ray based room acoustic simulations of small rooms (Vol. 12). Logos Verlag Berlin GmbH.

Asadollahi, M. (2017). Finite volume method for poroelasticity.

Bailey, C., Taylor, G., Cross, M., \& Chow, P. (1999). Discretisation procedures for multiphysics phenomena. Journal of Computational and Applied Mathematics, 103(1), $3-17$.

Baliga, B., \& Patankar, S. (1980). A new finite-element formulation for convectiondiffusion problems. Numerical Heat Transfer, 3(4), 393-409.

Bao, H., Bielak, J., Ghattas, O., Kallivokas, L. F., O’Hallaron, D. R., Shewchuk, J. R., \& Xu, J. (1998). Large-scale simulation of elastic wave propagation in heterogeneous media on parallel computers. Computer methods in applied mechanics and engineering, 152(1-2), 85-102.

Bašić, H., Demirdžić, I., \& Muzaferija, S. (2005). Finite volume method for simulation of extrusion processes. International journal for numerical methods in engineering, 62(4), 475-494.

Batzle, M. L., Han, D.-H., \& Hofmann, R. (2006). Fluid mobility and frequencydependent seismic velocity—direct measurements. Geophysics, 71(1), N1-N9.

Benjemaa, M., Glinsky-Olivier, N., Cruz-Atienza, V., Virieux, J., \& Piperno, S. (2007). Dynamic non-planar crack rupture by a finite volume method. Geophysical Journal International, 171(1), 271-285.

Berezovski, A., \& Maugin, G. (2001). Simulation of thermoelastic wave propagation by means of a composite wave-propagation algorithm. Journal of Computational Physics, 168(1), 249-264.

Berezovski, A., \& Maugin, G. A. (2003). Simulation of wave and front propagation in thermoelastic materials with phase transformation. Computational materials science, $28(3-4), 478-485$.

Binns, K. J., \& Lawrenson, P. J. (2013). Analysis and computation of electric and magnetic field problems: Pergamon international library of science, technology, engineering and social studies. Elsevier.

Biot, M. A. (1956). Theory of propagation of elastic waves in a fluid-saturated porous 
solid. ii. higher frequency range. The Journal of the acoustical Society of america, 28(2), 179-191.

Biot, M. A. (1962). Generalized theory of acoustic propagation in porous dissipative media. The Journal of the Acoustical Society of America, 34(9A), 1254-1264.

Boole, G. (1880). A treatise on the calculus of finite differences. Macmillan and company.

Borish, J. (1984). Extension of the image model to arbitrary polyhedra. The Journal of the Acoustical Society of America, 75(6), 1827-1836.

Boscheri, W., Dumbser, M., \& Loubère, R. (2016). Cell centered direct arbitrarylagrangian-eulerian ader-weno finite volume schemes for nonlinear hyperelasticity. Computers \& Fluids, 134, 111-129.

Botts, J., \& Savioja, L. (2013). Integrating finite difference schemes for scalar and vector wave equations. In 2013 ieee international conference on acoustics, speech and signal processing (pp. 171-175).

Bressan, J., Martins, M., \& Vaz Jr, M. (2010). Stress evolution and thermal shock computation using the finite volume method. Journal of Thermal Stresses, 33(6), 533558.

Bressan, J. D., Martins, M. M., \& Button, S. T. (2017). Analysis of metal extrusion by the finite volume method. Procedia Engineering, 207, 425-430.

Brossier, R., Virieux, J., \& Operto, S. (2008). Parsimonious finite-volume frequencydomain method for 2-dp-sv-wave modelling. Geophysical Journal International, 175(2), 541-559.

Brown, R. L. (2009). Anomalous dispersion due to hydrocarbons: The secret of reservoir geophysics? The Leading Edge, 28(4), 420-425.

Bryant, E. C., Hwang, J., Sharma, M. M., et al. (2015). Arbitrary fracture propagation in heterogeneous poroelastic formations using a finite volume-based cohesive zone model. In Spe hydraulic fracturing technology conference.

Buchmüller, P., Dreher, J., \& Helzel, C. (2016). Improved accuracy of high-order weno finite volume methods on cartesian grids with adaptive mesh refinement. In $X v i$ international conference on hyperbolic problems: Theory, numerics, applications (pp. 263-272). 
Burden, R. L., \& Faires, J. D. (2001). Numerical analysis 7th edition. Thomson Learning ISBN 0-534-38216-9.

Carcione, J. M. (2007). Wave fields in real media: Wave propagation in anisotropic, anelastic, porous and electromagnetic media (Vol. 38). Elsevier.

Cardiff, P. (2012). Development of the finite volume method for hip joint stress analysis.

Cardiff, P., \& Demirdžić, I. (2018). Thirty years of the finite volume method for solid mechanics. arXiv preprint arXiv:1810.02105.

Cardiff, P., Karač, A., \& Ivanković, A. (2014). A large strain finite volume method for orthotropic bodies with general material orientations. Computer Methods in Applied Mechanics and Engineering, 268, 318-335.

Cardiff, P., Manchanda, R., Bryant, E. C., Lee, D., Ivankovic, A., \& Sharma, M. M. (2015). Simulation of fractures in openfoam: from adhesive joints to hydraulic fractures. In 10th openfoam workshop.

Cardiff, P., Tang, T., Tukovic, Z., Jasak, H., Ivankovic, A., \& De Jaeger, P. (2017). An eulerian-inspired lagrangian finite volume method for wire drawing simulations. In Iutam symposium on multi-scale fatigue, fracture and damage of materials in harsh environments. galway, ireland: National university of ireland galway.

Cardiff, P., Tuković, Ž., Jasak, H., \& Ivanković, A. (2016). A block-coupled finite volume methodology for linear elasticity and unstructured meshes. Computers \& structures, $175,100-122$.

Carolan, D., Tuković, Ž., Murphy, N., \& Ivanković, A. (2013). Arbitrary crack propagation in multi-phase materials using the finite volume method. Computational materials science, 69, 153-159.

Castañar, I., Baiges, J., \& Codina, R. (2019). A stabilized mixed finite element approximation for incompressible finite strain solid dynamics using a total lagrangian formulation.

Castillo, J. E. (1991). Mathematical aspects of numerical grid generation. SIAM.

Chaljub, E., Komatitsch, D., Vilotte, J.-P., Capdeville, Y., Valette, B., \& Festa, G. (2007). Spectral-element analysis in seismology. Advances in geophysics, 48, 365-419.

Chapman, M., Chesnokov, E. M., Sandhya Devi, K., \& Grechka, V. (2009). Rainbow in 
the earth-introduction. Society of Exploration Geophysicists.

Chapman, M., Liu, E., \& Li, X.-Y. (2006). The influence of fluid sensitive dispersion and attenuation on avo analysis. Geophysical Journal International, 167(1), 89-105.

Chen, Z., Lou, Z., \& Ruan, X. (2007). Finite volume simulation and mould optimization of aluminum profile extrusion. Journal of Materials Processing Technology, 190(13), 382-386.

Cheng, J.-B., Huang, W., Jiang, S., \& Tian, B. (2017). A third-order moving mesh cell-centered scheme for one-dimensional elastic-plastic flows. Journal of Computational Physics, 349, 137-153.

Cheng, J.-B., Jia, Y., Jiang, S., Toro, E. F., \& Yu, M. (2017). A second-order cell-centered lagrangian method for two-dimensional elastic-plastic flows. Communications in Computational Physics, 22(5), 1224-1257.

Cheng, J.-B., Toro, E. F., Jiang, S., Yu, M., \& Tang, W. (2015). A high-order cellcentered lagrangian scheme for one-dimensional elastic-plastic problems. Computers \& Fluids, 122, 136-152.

Clayton, R., \& Engquist, B. (1977). Absorbing boundary conditions for acoustic and elastic wave equations. Bulletin of the seismological society of America, 67(6), 1529-1540.

Cohen, G. (2013). Higher-order numerical methods for transient wave equations. Springer Science \& Business Media.

Cook, R. D., et al. (2007). Concepts and applications of finite element analysis. John Wiley \& Sons.

Coralic, V., \& Colonius, T. (2014). Finite-volume weno scheme for viscous compressible multicomponent flows. Journal of computational physics, 274, 95-121.

Courant, R. (1928). On the partial difference equations of mathematical physics. Mathematische Annalen, 100, 32-74.

Courant, R., et al. (1943). Variational methods for the solution of problems of equilibrium and vibrations. Verlag nicht ermittelbar.

Craggs, A. (1994). A finite element method for the free vibration of air in ducts and rooms with absorbing walls. Journal of Sound Vibration, 173, 568-576. 
Das, S., Mathur, S. R., \& Murthy, J. Y. (2012). Finite-volume method for creep analysis of thin rf mems devices using the theory of plates. Numerical Heat Transfer, Part B: Fundamentals, 61(2), 71-90.

Davies, D., \& Salmond, D. J. (1985). Calculation of the volume of a general hexahedron for flow predictions. AIAA journal, 23(6), 954-956.

Davis, P., \& Rabinowitz, P. (1956). Abscissas and weights for gaussian quadratures of high order. J. Res. Nat. Bur. Standards, 56(1), 35-37.

De Brauer, A., Iollo, A., \& Milcent, T. (2016). A cartesian scheme for compressible multimaterial models in 3d. Journal of Computational Physics, 313, 121-143.

De Brauer, A., Iollo, A., \& Milcent, T. (2017). A cartesian scheme for compressible multimaterial hyperelastic models with plasticity. Communications in Computational Physics, 22(5), 1362-1384.

de la Puente, J., Ampuero, J.-P., \& Käser, M. (2009). Dynamic rupture modeling on unstructured meshes using a discontinuous galerkin method. Journal of Geophysical Research: Solid Earth, 114(B10).

de la Puente, J., Dumbser, M., Käser, M., \& Igel, H. (2008). Discontinuous galerkin methods for wave propagation in poroelastic media. Geophysics, 73(5), T77-T97.

de la Puente, J., Käser, M., Dumbser, M., \& Igel, H. (2007). An arbitrary highorder discontinuous galerkin method for elastic waves on unstructured meshes-iv. anisotropy. Geophysical Journal International, 169(3), 1210-1228.

Demirdžić, I., Džaferović, E., \& Ivanković, A. (2005). Finite-volume approach to thermoviscoelasticity. Numerical heat transfer, Part B, 47(3), 213-237.

Demirdžić, I., Horman, I., \& Martinović, D. (2000). Finite volume analysis of stress and deformation in hygro-thermo-elastic orthotropic body. Computer methods in applied mechanics and engineering, 190(8-10), 1221-1232.

Demirdžić, I., \& Martinović, D. (1993). Finite volume method for thermo-elasto-plastic stress analysis. Computer methods in applied mechanics and engineering, 109(3-4), $331-349$.

Demirdžic, I., Martinovic, D., \& Ivankovic, A. (1988). Numerical simulation of thermal deformation in welded workpiece. Zavarivanje. 
Demirdžić, I., \& Muzaferija, S. (1994). Finite volume method for stress analysis in complex domains. International journal for numerical methods in engineering, 37(21), 3751-3766.

Demirdžić, I., \& Muzaferija, S. (1995). Numerical method for coupled fluid flow, heat transfer and stress analysis using unstructured moving meshes with cells of arbitrary topology. Computer methods in applied mechanics and engineering, 125(1-4), 235255.

de Souza Araujo, A. L., Fernandes, B. R. B., Araujo, R. M., Drumond Filho, E. P., Lima, I. d. C. M., Marcondes, F., \& Sepehrnoori, K. (2013). 3d compositional reservoir simulation using unstructured grids in homogeneous reservoirs.

Després, B., \& Labourasse, E. (2015). Angular momentum preserving cell-centered lagrangian and eulerian schemes on arbitrary grids. Journal of Computational Physics, 290, 28-54.

Dioh, N., Ivankovic, A., Leevers, P., \& Williams, J. (1994). The high strain rate behavior of polymers. Les Editions de Physique Les Ulis, J. Phys.(France) IV(France), 4, $119-124$.

Dormy, E., \& Tarantola, A. (1995). Numerical simulation of elastic wave propagation using a finite volume method. Journal of Geophysical Research: Solid Earth, 100(B2), 2123-2133.

Duffy, D. G. (2015). Green's functions with applications. Chapman and Hall/CRC.

Dumbser, M., Hidalgo, A., Castro, M., Parés, C., \& Toro, E. F. (2010). Force schemes on unstructured meshes ii: Non-conservative hyperbolic systems. Computer Methods in Applied Mechanics and Engineering, 199(9-12), 625-647.

Dumbser, M., Hidalgo, A., \& Zanotti, O. (2014). High order space-time adaptive aderweno finite volume schemes for non-conservative hyperbolic systems. Computer Methods in Applied Mechanics and Engineering, 268, 359-387.

Dumbser, M., \& Käser, M. (2006). An arbitrary high-order discontinuous galerkin method for elastic waves on unstructured meshes-ii. the three-dimensional isotropic case. Geophysical Journal International, 167(1), 319-336.

Dumbser, M., Zanotti, O., Hidalgo, A., \& Balsara, D. S. (2013). Ader-weno finite volume 
schemes with space-time adaptive mesh refinement. Journal of Computational Physics, 248, 257-286.

Durran, D. R. (2013). Numerical methods for wave equations in geophysical fluid dynamics (Vol. 32). Springer Science \& Business Media.

Dvorkin, J., Mavko, G., \& Nur, A. (1995). Squirt flow in fully saturated rocks. Geophysics, 60(1), 97-107.

Ebrom, D. (2004). The low-frequency gas shadow on seismic sections. The Leading Edge, 23(8), 772-772.

Epperson, J. F. (2013). An introduction to numerical methods and analysis. John Wiley \& Sons.

Etgen, J. T. (2007). A tutorial on optimizing time domain finite-difference schemes:"beyond holberg". Stanford Exploration Project Report, 129, 33-43.

Etienne, V., Chaljub, E., Virieux, J., \& Glinsky, N. (2010). An hp-adaptive discontinuous galerkin finite-element method for 3-d elastic wave modelling. Geophysical Journal International, 183(2), 941-962.

Faccioli, E., Maggio, F., Paolucci, R., \& Quarteroni, A. (1997). 2d and 3d elastic wave propagation by a pseudo-spectral domain decomposition method. Journal of seismology, 1(3), 237-251.

Fainberg, J., \& Leister, H.-J. (1996). Finite volume multigrid solver for thermo-elastic stress analysis in anisotropic materials. Computer Methods in Applied Mechanics and Engineering, 137(2), 167-174.

Ferguson, W. J. (1995). A control volume finite element numerical simulation of the high temperature drying of spruce. Drying Technology, 13(3), 607-634.

Ferguson, W. J., \& Turner, I. (1996). A control volume finite element numerical simulation of the drying of spruce. Journal of Computational Physics, 125(1), 59-70.

Ferziger, J., \& Peric, M. (2012). Computational methods for fluid dynamics: Springer science \& business media.

Filippini, G., Maliska, C., \& Vaz Jr, M. (2014). A physical perspective of the elementbased finite volume method and fem-galerkin methods within the framework of the space of finite elements. International Journal for Numerical Methods in Engineer- 
ing, 98(1), 24-43.

Forsythe, G. E., Wasow, W. R., et al. (1960). Finite-difference methods for partial differential equations.

Fridrich, D., Liska, R., \& Wendroff, B. (2017). Cell-centered lagrangian lax-wendroff hll hybrid method for elasto-plastic flows. Computers \& Fluids, 157, 164-174.

Fryer, Y., Bailey, C., Cross, M., \& Lai, C.-H. (1991). A control volume procedure for solving the elastic stress-strain equations on an unstructured mesh. Applied mathematical modelling, 15(11-12), 639-645.

Futterman, W. I. (1962). Dispersive body waves. Journal of Geophysical research, 67(13), 5279-5291.

Ganley, D. (1981). A method for calculating synthetic seismograms which include the effects of absorption and dispersion. Geophysics, 46(8), 1100-1107.

Geertsma, J., \& Smit, D. (1961). Some aspects of elastic wave propagation in fluidsaturated porous solids. Geophysics, 26(2), 169-181.

George, P. L. (1992). Automatic mesh generation: applications to finite element methods. John Wiley \& Sons, Inc.

Georges, G., Breil, J., \& Maire, P.-H. (2017). A 3d finite volume scheme for solving the updated lagrangian form of hyperelasticity. International Journal for Numerical Methods in Fluids, 84(1), 41-54.

Godlewski, E., \& Raviart, P.-A. (1996). Applied mathematical science. Numerical approximation of hyperbolic systems of conservation laws, 118.

Godunov, S. K. (1959). A difference method for numerical calculation of discontinuous solutions of the equations of hydrodynamics. Matematicheskii Sbornik, 89(3), 271306.

Godunov, S. K. (1962). The problem of a generalized solution in the theory of quasilinear equations and in gas dynamics. Russian Mathematical Surveys, 17(3), 145.

Goloshubin, G. M., \& Bakulin, A. V. (1998). Seismic reflectivity of a thin porous fluidsaturated layer versus frequency. In Seg technical program expanded abstracts 1998 (pp. 976-979). Society of Exploration Geophysicists.

Goloshubin, G. M., \& Korneev, V. A. (2000). Seismic low-frequency effects from fluid- 
saturated reservoir. In Seg technical program expanded abstracts 2000 (pp. 16711674). Society of Exploration Geophysicists.

Goloshubin, G. M., \& Korneev, V. A. (2005, September 6). Frequency-dependent processing and interpretation (fdpi) of seismic data for identifying, imaging and monitoring fluid-saturated underground reservoirs. Google Patents. (US Patent $6,941,227)$

Goloshubin, G. M., Korneev, V. A., \& Vingalov, V. M. (2002). Seismic low-frequency effects from oil-saturated reservoir zones. In Seg technical program expanded abstracts 2002 (pp. 1813-1816). Society of Exploration Geophysicists.

Goloshubin, G. M., Verkhovsky, A. M., \& Kaurov, V. V. (1996). Laboratory experiments of seismic monitoring. In 58th eage conference and exhibition.

Gottlieb, S., \& Shu, C.-W. (1998). Total variation diminishing runge-kutta schemes. Mathematics of computation of the American Mathematical Society, 67(221), 7385.

Greenshields, C., Venizelos, G., \& Ivanković, A. (2000). A fluid-structure model for fast brittle fracture in plastic pipes. Journal of Fluids and Structures, 14(2), 221-234.

Greenshields, C., \& Weller, H. (2005). A unified formulation for continuum mechanics applied to fluid-structure interaction in flexible tubes. International Journal for Numerical Methods in Engineering, 64(12), 1575-1593.

Greenshields, C., Weller, H., \& Ivanković, A. (1999a). A finite volume formulation for fluid structure interaction. In Second international symposium on finites volumes for complex applications: Problems and perspectives, duisburg, germany.

Greenshields, C., Weller, H., \& Ivanković, A. (1999b). The finite volume method for coupled fluid flow and stress analysis. Computer Modeling and Simulation in Engineering, 4(3), 213-218.

Habchi, C., Russeil, S., Bougeard, D., Harion, J.-L., Lemenand, T., Ghanem, A., ... Peerhossaini, H. (2013). Partitioned solver for strongly coupled fluid-structure interaction. Computers \& Fluids, 71, 306-319.

Haider, J., Lee, C. H., Gil, A. J., Huerta, A., \& Bonet, J. (2018). An upwind cell centred total lagrangian finite volume algorithm for nearly incompressible explicit fast solid 
dynamic applications. Computer Methods in Applied Mechanics and Engineering, $340,684-727$.

Harlow, F. H., \& Welch, J. E. (1965). Numerical calculation of time-dependent viscous incompressible flow of fluid with free surface. The physics of fluids, 8(12), 21822189.

Harten, A., Lax, P. D., \& Leer, B. v. (1983). On upstream differencing and godunov-type schemes for hyperbolic conservation laws. SIAM review, 25(1), 35-61.

Hattel, J. (1993a). Control volume based finite difference method,-numerical simulation of thermal and mechanical conditions in casting and heat treatment processes.

Hattel, J. (1993b). Stress calculations using a control volume based finite difference method. Revnedannelse og Brudmekanik, DMS Vintermфdeårbog, 1993.

Hattel, J., Hansen, P., \& Andersen, S. (1993). Modelling of thermally induced stresses in high pressure die casting dies. In 19th international nadca die casting congress.

Hattel, J., Hansen, P., \& Hansen, L. F. (1993). Analysis of thermally induced stresses in die casting using a novel control volume technique. In Mcwasp vi.

Hattel, J., \& Pryds, N. (2001). Modelling rapid solidification with the control volume method. In 22nd ris $\phi$ international symposium on materials science.

Hattel, J. H. (1997). Numerical modelling of stresses and deformations in casting processes. In Casting 1997, int. adi and simulation conference.

Hattel, J. H., \& Thorborg, J. (2003). A numerical model for predicting the thermomechanical conditions during hydration of early-age concrete. Applied Mathematical Modelling, 27(1), 1-26.

Hattel, J. H., Thorborg, J., \& Andersen, S. (1998). Stress/strain modelling of casting processes in the framework of the control-volume method. In Modeling of casting and advanced solidification processes viii.

Hattet, J., \& Hansen, P. (1990). Fdm solutions of the thermoe-lastic equations using a staggered grid. In Danish-german-polish workshop on application of computer methods in practice.

He, Z., Xiong, X., \& Bian, L. (2008). Numerical simulation of seismic low-frequency shadows and its application. Applied Geophysics, 5(4), 301-306. 
Heinrich, B. (1987). Boundary value problems and irregular networks. In Finite difference methods on irregular networks (pp. 17-39). Springer.

Hejranfar, K., \& Azampour, M.-H. (2015). Assessment of cell-centered and cell-vertex finite volume approaches for computation of $2 \mathrm{~d}$ structural dynamics on arbitrary quadrilateral grids. CMES: Computer Modeling in Engineering \& Sciences, 106(6), $395-439$.

Henry, F., \& Collins, M. (1970). Prediction of transient wall movement of an incompressible elastic tube using a finite volume procedure. WIT Transactions on Biomedicine and Health, 1.

Henry, F., \& Collins, M. (1993). A novel predictive model with compliance for arterial flows. ASME-PUBLICATIONS-BED, 26, 131-131.

Hermeline, F. (1993). Two coupled particle-finite volume methods using delaunayvorono1 meshes for the approximation of vlasov-poisson and vlasov-maxwell equations. Journal of Computational Physics, 106(1), 1-18.

Hernández, J. (2002). High-order finite volume schemes for the advection-diffusion equation. International Journal for Numerical Methods in Engineering, 53(5), 12111234.

Heuzé, T. (2017). Lax-wendroff and tvd finite volume methods for unidimensional thermomechanical numerical simulations of impacts on elastic-plastic solids. Journal of Computational Physics, 346, 369-388.

Heuzé, T. (2018). Simulation of impacts on elastic-viscoplastic solids with the fluxdifference splitting finite volume method applied to non-uniform quadrilateral meshes. Advanced Modeling and Simulation in Engineering Sciences, 5(1), 9.

Hidalgo, A., \& Dumbser, M. (2011). Ader schemes for nonlinear systems of stiff advection-diffusion-reaction equations. Journal of Scientific Computing, 48(1-3), $173-189$.

Horman, I., Hajdarević, S., Martinović, S., \& Vukas, N. (2010). Numerical analysis of stress and strain in a wooden chair. Drvna industrija: Znanstveni časopis za pitanja drvne tehnologije, 61(3), 151-158.

Horman, I., Martinović, D., Bijelonja, I., \& Hajdarević, S. (2012). Wood subjected to 
hygro-thermal and/or mechanical loads. Finite Volume Method: Powerful Means of Engineering Design, 327.

Hosken, J. (1988). Ricker wavelets in their various guises. First Break, 6(1), 24-33.

Howell, B., \& Ball, G. (2002). A free-lagrange augmented godunov method for the simulation of elastic-plastic solids. Journal of Computational Physics, 175(1), 128167.

Hyman, J. M., Knapp, R. J., \& Scovel, J. C. (1992). High order finite volume approximations of differential operators on nonuniform grids. Physica D: Nonlinear Phenomena, 60(1-4), 112-138.

Igel, H., Nissen-Meyer, T., \& Jahnke, G. (2002). Wave propagation in 3d spherical sections: effects of subduction zones. Physics of the Earth and Planetary Interiors, 132(1-3), 219-234.

Ihlenburg, F. (2006). Finite element analysis of acoustic scattering (Vol. 132). Springer Science \& Business Media.

Ivanković, A., Karač, A., Dendrinos, E., \& Parker, K. (2001). Blood flow in deformable arteries: the finite volume method for fluid-structure interaction problem. In Proc. 9th acme conference on computational mechanics in engineering.

Ivanković, A., Karač, A., Dendrinos, E., \& Parker, K. (2002). Towards early diagnosis of atherosclerosis: the finite volume method for fluid-structure interaction. Biorheology, 39(3, 4), 401-407.

Jafari, M. R., Zebarjad, S. M., \& Kolahan, F. (2007). Simulation of thixoformability of a356 aluminum alloy using finite volume method. Materials Science and Engineering: A, 454, 558-563.

Jagad, P. (2016). A numerical procedure for elastic solids. GIT-Journal of Engineering and Technology, 9, 113-124.

Jagad, P., Puranik, B., \& Date, A. (2011). A finite volume procedure on unstructured meshes for fluid-structure interaction problems. World Academy of Science, Engineering and Technology, Paris, France, 639-645.

Jagad, P., Puranik, B., \& Date, A. (2017). A numerical analysis of fluid-structure interaction problem with a flow channel embedded in a structural material. In Proc indian 
natn sci acad (Vol. 83, pp. 655-667).

Jagad, P., Puranik, B., \& Date, A. (2018). A finite volume procedure for fluid flow, heat transfer and solid-body stress analysis. International Journal for Computational Methods in Engineering Science and Mechanics, 19(3), 171-184.

Jasak, H., Jemcov, A., Tukovic, Z., et al. (2007). Openfoam: A c++ library for complex physics simulations. In International workshop on coupled methods in numerical dynamics (Vol. 1000, pp. 1-20).

Jayantha, P. A., \& Turner, I. W. (2001). A comparison of gradient approximations for use in finite-volume computational models for two-dimensional diffusion equations. Numerical Heat Transfer, Part B: Fundamentals, 40(5), 367-390.

Jayantha, P. A., \& Turner, I. W. (2003). On the use of surface interpolation techniques in generalised finite volume strategies for simulating transport in highly anisotropic porous media. Journal of computational and applied mathematics, 152(1-2), 199216.

Kanyanta, V., Ivankovic, A., \& Karac, A. (2009). Validation of a fluid-structure interaction numerical model for predicting flow transients in arteries. Journal of Biomechanics, 42(11), 1705-1712.

Käser, M., \& Dumbser, M. (2006). An arbitrary high-order discontinuous galerkin method for elastic waves on unstructured meshes- $\mathrm{i}$. the two-dimensional isotropic case with external source terms. Geophysical Journal International, 166(2), 855-877.

Käser, M., Igel, H., Sambridge, M., \& Braun, J. (2001). A comparative study of explicit differential operators on arbitrary grids. Journal of Computational Acoustics, 9(03), $1111-1125$.

Kelly, K. R., Ward, R. W., Treitel, S., \& Alford, R. M. (1976). Synthetic seismograms: A finite-difference approach. Geophysics, 41(1), 2-27.

Kluth, G., \& Després, B. (2008). Fv schemes for hyperelastic-plastic models in finite deformations. Finite volumes for complex applications $V, 511-518$.

Kluth, G., \& Després, B. (2010). Discretization of hyperelasticity on unstructured mesh with a cell-centered lagrangian scheme. Journal of Computational Physics, 229(24), 9092-9118. 
Komatitsch, D., Barnes, C., \& Tromp, J. (2000). Wave propagation near a fluid-solid interface: A spectral-element approach. Geophysics, 65(2), 623-631.

Komatitsch, D., \& Tromp, J. (1999). Introduction to the spectral element method for three-dimensional seismic wave propagation. Geophysical journal international, $139(3), 806-822$.

Komatitsch, D., \& Vilotte, J.-P. (1998). The spectral element method: an efficient tool to simulate the seismic response of $2 \mathrm{~d}$ and $3 \mathrm{~d}$ geological structures. Bulletin of the seismological society of America, 88(2), 368-392.

Korneev, V. (2007). Slow waves in fractures filled with viscous fluid. Geophysics, 73(1), N1-N7.

Korneev, V. A., Goloshubin, G. M., Daley, T. M., \& Silin, D. B. (2004). Seismic lowfrequency effects in monitoring fluid-saturated reservoirs. Geophysics, 69(2), 522532.

Kovacevic, A., Stosic, N., Mujic, E., \& Smith, I. K. (2007). Cfd integrated design of screw compressors. Engineering Applications of Computational Fluid Mechanics, 1(2), 96-108.

Kovacevic, A., Stosic, N., \& Smith, I. (2007). Screw compressors: three dimensional computational fluid dynamics and solid fluid interaction (Vol. 46). Springer Science \& Business Media.

Kovacevic, A., Stosic, N., \& Smith, I. K. (2004). A numerical study of fluid-solid interaction in screw compressors. International Journal on Computer Application in Technology, 21(4), 148-158.

Kovačević, A., Stošić, N., \& Smith, I. K. (2006). Numerical simulation of combined screw compressor-expander machines for use in high pressure refrigeration systems. Simulation Modelling Practice and Theory, 14(8), 1143-1154.

Krokstad, A., Strom, S., \& Sørsdal, S. (1968). Calculating the acoustical room response by the use of a ray tracing technique. Journal of Sound and Vibration, 8(1), 118125.

Kuttruff, H. (2016). Room acoustics. Crc Press.

Laine, S., Siltanen, S., Lokki, T., \& Savioja, L. (2009). Accelerated beam tracing algo- 
rithm. Applied Acoustics, 70(1), 172-181.

Lee, C. H., Gil, A. J., \& Bonet, J. (2013). Development of a cell centred upwind finite volume algorithm for a new conservation law formulation in structural dynamics. Computers \& Structures, 118, 13-38.

Lee, D., Cardiff, P., Bryant, E. C., Manchanda, R., Wang, H., Sharma, M. M., et al. (2015). A new model for hydraulic fracture growth in unconsolidated sands with plasticity and leak-off. In Spe annual technical conference and exhibition.

Leonard, M., Murphy, N., Karač, A., \& Ivanković, A. (2012). A numerical investigation of spherical void growth in an elastic-plastic continuum. Computational Materials Science, 64, 38-40.

LeVeque, R. J. (2002). Finite volume methods for hyperbolic problems (Vol. 31). Cambridge university press.

Lilek, Ž., Muzaferija, S., Perić, M., \& Seidl, V. (1997). Computation of unsteady flows using nonmatching blocks of structured grid. Numerical Heat Transfer, 32(4), 403418.

Liu, F., Turner, I., \& Anh, V. (2002). An unstructured mesh finite volume method for modelling saltwater intrusion into coastal aquifers. Korean Journal of Computational \& Applied Mathematics, 9(2), 391-407.

Liu, X.-D., Osher, S., Chan, T., et al. (1994). Weighted essentially non-oscillatory schemes. Journal of computational physics, 115(1), 200-212.

Liu, Y. (2004). Seismic "low frequency shadows" for gas sand reflection. In Seg technical program expanded abstracts 2004 (pp. 1563-1566). Society of Exploration Geophysicists.

Lou, S., Zhao, G., Wang, R., \& Wu, X. (2008). Modeling of aluminum alloy profile extrusion process using finite volume method. Journal of Materials Processing Technology, 206(1-3), 481-490.

Loubère, R., Maire, P.-H., \& Rebourcet, B. (2016). Staggered and colocated finite volume schemes for lagrangian hydrodynamics. In Handbook of numerical analysis (Vol. 17, pp. 319-352). Elsevier.

Love, C. H. (1966). Abscissas and weights for gaussian quadrature. 
Ma, T., Li, P., \& Ma, T. (2020). A three-dimensional cartesian mesh generation algorithm based on the gpu parallel ray casting method. Applied Sciences, 10(1), 58.

MacCormack, R., \& Paullay, A. (1972). Computational efficiency achieved by time splitting of finite difference operators. In 10th aerospace sciences meeting (p. 154).

Maire, P.-H., Abgrall, R., Breil, J., Loubère, R., \& Rebourcet, B. (2013). A nominally second-order cell-centered lagrangian scheme for simulating elastic-plastic flows on two-dimensional unstructured grids. Journal of Computational Physics, 235, $626-665$.

Manchanda, R., Bryant, E. C., Bhardwaj, P., Sharma, M. M., et al. (2016). Strategies for effective stimulation of multiple perforation clusters in horizontal wells. In Spe hydraulic fracturing technology conference.

Maneeratana, K. (2000). Development of the finite volume method for non-linear structural applications.

Manzini, G., \& Ferraris, S. (2004). Mass-conservative finite volume methods on 2-d unstructured grids for the richards' equation. Advances in Water Resources, 27(12), 1199-1215.

Martinović, D., Horman, I., \& Demirdžić, I. (2001). Numerical and experimental analysis of a wood drying process. Wood science and technology, 35(1-2), 143-156.

Martinović, D., Horman, I., \& Hajdarević, S. (2008). Stress distribution in wooden corner joints (raspodjela naprezanja u drvenim kutnim spojevima). Strojarstvo, 50, 193-204.

Martins, M. M., Bressan, J. D., \& Button, S. T. (2017). Finite volume analysis with the maccormack method applied to metal flow in forward extrusion. Universal Journal of Mechanical Engineering, 5(1), 1-8.

Martins, M. M., Bressan, J. D., Button, S. T., \& Ivankovic, A. (2011). Extrusion process by finite volume method using openfoam software. In Aip conference proceedings (Vol. 1315, pp. 1461-1466).

Matos Martins, M., Button, S. T., \& Bressan, J. D. (2016). Analysis of aluminum extrusion in a 90o die by finite volume method. In Advanced materials research (Vol. 1135, pp. 153-160). 
Mavko, G., \& Mukerji, T. (1998). Bounds on low-frequency seismic velocities in partially saturated rocks. Geophysics, 63(3), 918-924.

McDonald, P. (1971). The computation of transonic flow through two-dimensional gas turbine cascades. In Asme 1971 international gas turbine conference and products show.

Mensah, V., Hidalgo, A., \& Ferro, R. M. (2019). Numerical modelling of the propagation of diffusive-viscous waves in a fluid-saturated reservoir using finite volume method. Geophysical Journal International, 218(1), 33-44.

Miller, G. H., \& Puckett, E. G. (1996). A high-order godunov method for multiple condensed phases. Journal of Computational Physics, 128(1), 134-164.

Minkowycz, W., Sparrow, E. M., Schneider, G., \& Pletcher, R. (1988). Handbook of numerical heat transfer. New York, Wiley-Interscience, 1988, 1035 p. No individual items are abstracted in this volume..

Mitchell, A., \& WAIT, R. (1977). The finite element method in partial differential equations(book). London and New York, Wiley-Interscience, 1977. 205 p.

Ndanou, S., Favrie, N., \& Gavrilyuk, S. (2015). Multi-solid and multi-fluid diffuse interface model: Applications to dynamic fracture and fragmentation. Journal of Computational Physics, 295, 523-555.

Niceno, B. (2003). An unstructured parallel algorithm for large eddy and conjugate heat transfer simulations.

Noelle, S., Xing, Y., \& Shu, C.-W. (2007). High-order well-balanced finite volume weno schemes for shallow water equation with moving water. Journal of Computational Physics, 226(1), 29-58.

Noisternig, M., Katz, B. F., Siltanen, S., \& Savioja, L. (2008). Framework for real-time auralization in architectural acoustics. Acta Acustica United with Acustica, 94(6), 1000-1015.

Okuzono, T., Yoshida, T., Sakagami, K., \& Otsuru, T. (2016). An explicit time-domain finite element method for room acoustics simulations: Comparison of the performance with implicit methods. Applied Acoustics, 104, 76-84.

Ollivier-Gooch, C., \& Van Altena, M. (2002). A high-order-accurate unstructured mesh 
finite-volume scheme for the advection-diffusion equation. Journal of Computational Physics, 181(2), 729-752.

Papadakis, G. (2008). A novel pressure-velocity formulation and solution method for fluid-structure interaction problems. Journal of computational physics, 227(6), 3383-3404.

Papadakis, G., \& Giannopapa, C. (2006). Towards a unified solution method for fluidstructure interaction problems: progress and challenges. In Proceedings of 2006 asme pressure vessels and piping division conference.

Pasdunkorale Arachchige, J. (2003). Accurate finite volume methods for the numerical simulation of transport in highly anistropic media (Unpublished doctoral dissertation). Queensland University of Technology.

Patankar, S. (1980). Numerical heat transfer and fluid flow: Crc press.

Peiró, J., \& Sherwin, S. (2005). Finite difference, finite element and finite volume methods for partial differential equations. In Handbook of materials modeling (pp. 24152446). Springer.

Perre, p., \& Passard, J. (1995). A control-volume procedure compared with the finiteelement method for calculating stress and strain during wood drying. Drying Technology, 13(3), 635-660.

Pierce, A. D., \& Beyer, R. T. (1990). Acoustics: An introduction to its physical principles and applications. 1989 edition. ASA.

Pryds, N., \& Hattel, J. H. (1997). Numerical modelling of rapid solidification. Modelling and Simulation in Materials Science and Engineering, 5(5), 451.

Rajagopal, K., Srinivasa, A., \& Ponnalagu, A. (2014). Thermo-inelastic response of polymeric solids (Tech. Rep.). TEXAS ENGINEERING EXPERIMENT STATION COLLEGE STATION.

Regan, H. M. (2002). Von neumann stability analysis of symplectic integrators applied to hamiltonian pdes. Journal of Computational Mathematics, 611-618.

Rente, C., \& Oliveira, P. (2000). Extension of a finite volume method in solid stress analysis to cater for non-linear elasto-plastic effects. In Proceedings of em 14th engineering mechanics conference, university of texas at austin, usa. 
Richardson, L. F. (1911). Ix. the approximate arithmetical solution by finite differences of physical problems involving differential equations, with an application to the stresses in a masonry dam. Philosophical Transactions of the Royal Society of London. Series A, Containing Papers of a Mathematical or Physical Character, 210(459-470), 307-357.

Ricker, N. (1943). Further developments in the wavelet theory of seismogram structure. Bulletin of the Seismological Society of America, 33(3), 197-228.

Ricker, N. (1944). Wavelet functions and their polynomials. Geophysics, 9(3), 314-323.

Ricker, N. (1953). The form and laws of propagation of seismic wavelets. Geophysics, $18(1), 10-40$.

Ruiz, P. M. (1970). A technique for simulating the vibration of strings with a digital computer (Unpublished doctoral dissertation). University of Illinois at UrbanaChampaign.

Ryff, P. F., Biringer, P. P., \& Burke, P. E. (1970). Calculation methods for current distribution in single-turn coils of arbitrary cross section. IEEE Transactions on Power Apparatus and Systems(2), 228-232.

Saarelma, J., \& Savioja, L. (2014). An open source finite-difference time-domain solver for room acoustics using graphics processing units.

Safari, A., Tukovic, Z., Cardiff, P., Walter, M., Casey, E., \& Ivankovic, A. (2016). Interfacial separation of a mature biofilm from a glass surface-a combined experimental and cohesive zone modelling approach. Journal of the mechanical behavior of biomedical materials, 54, 205-218.

Sambasivan, S. K., Shashkov, M. J., \& Burton, D. E. (2013). A finite volume cell-centered lagrangian hydrodynamics approach for solids in general unstructured grids. International Journal for Numerical Methods in Fluids, 72(7), 770-810.

Sarma, M. (1970). Potential functions in electromagnetic field problems. IEEE Transactions on Magnetics, 6(3), 513-518.

Savioja, L., \& Svensson, U. P. (2015). Overview of geometrical room acoustic modeling techniques. The Journal of the Acoustical Society of America, 138(2), 708-730.

Schäfer, M., \& Teschauer, I. (2001). Numerical simulation of coupled fluid-solid prob- 
lems. Computer Methods in Applied Mechanics and Engineering, 190(28), 36453667.

Schäfer, M., Teschauer, I., Kadinski, L., \& Selder, M. (2002). A numerical approach for the solution of coupled fluid-solid and thermal stress problems in crystal growth processes. Computational materials science, 24(3), 409-419.

Schweig, E., \& Bridges, W. B. (1984). Computer analysis of dielectric waveguides: A finite-difference method. IEEE transactions on microwave theory and techniques, 32(5), 531-541.

Šekutkovski, B., Kostić, I., Simonović, A., Cardiff, P., \& Jazarević, V. (2016). Threedimensional fluid-structure interaction simulation with a hybrid rans-les turbulence model for applications in transonic flow domain. Aerospace Science and Technology, 49, 1-16.

Selmin, V. (1993). The node-centred finite volume approach: bridge between finite differences and finite elements. Computer Methods in Applied Mechanics and Engineering, 102(1), 107-138.

Sevilla, R., Giacomini, M., \& Huerta, A. (2018a). A face-centred finite volume method for second-order elliptic problems. International Journal for Numerical Methods in Engineering, 115(8), 986-1014.

Sevilla, R., Giacomini, M., \& Huerta, A. (2018b). A locking-free face-centred finite volume (fcfv) method for linear elasticity. arXiv preprint arXiv:1806.07500.

Shaw, G., Stone, T., et al. (2005). Finite volume methods for coupled stress/fluid flow in a commercial reservoir simulator. In Spe reservoir simulation symposium.

Sheaffer, J., Fazenda, B., et al. (2014). Wavecloud: an open source room acoustics simulator using the finite difference time domain method. In Forum acusticum.

Sheppard, W. F. (1899). Central-difference formulæ. Proceedings of the London Mathematical Society, 1(1), 449-488.

Shu, C.-W. (2003). High-order finite difference and finite volume weno schemes and discontinuous galerkin methods for cfd. International Journal of Computational Fluid Dynamics, 17(2), 107-118.

Shu, C.-W., \& Osher, S. (1988). Efficient implementation of essentially non-oscillatory 
shock-capturing schemes. Journal of computational physics, 77(2), 439-471.

Shuku, T., \& Ishihara, K. (1973). The analysis of the acoustic field in irregularly shaped rooms by the finite element method. Journal of Sound and Vibration, 29(1), 67IN1.

SICOT, F. (2006). High order schemes on non-uniform structured meshes in a finitevolume formulation (Unpublished doctoral dissertation). Thesis for Master of Science, Department of Mathematics, Chalmers University ....

Sijoy, C., \& Chaturvedi, S. (2015). An eulerian multi-material scheme for elastic-plastic impact and penetration problems involving large material deformations. European Journal of Mechanics-B/Fluids, 53, 85-100.

Smith, G. D., Smith, G. D., \& Smith, G. D. S. (1985). Numerical solution of partial differential equations: finite difference methods. Oxford university press.

Smith, I. K., Stosic, N., \& Kovacevic, A. (2014). Power recovery from low grade heat by means of screw expanders. Elsevier.

Song, S.-H., \& Chen, M.-Z. (2002). Third order accurate large-particle finite volume method on unstructured triangular meshes. SIAM Journal on Scientific Computing, 23(5), 1456-1463.

Sousa, E. (2003). The controversial stability analysis. Applied Mathematics and Computation, 145(2-3), 777-794.

Spanos, T. J. (2001). The thermophysics of porous media. Chapman and Hall/CRC.

Stephen, R. (1983). A comparison of finite difference and reflectivity seismograms for marine models. Geophysical Journal International, 72(1), 39-57.

Stoll, R. (1967). Numerical method of calculating eddy currents in nonmagnetic conductors. In Proceedings of the institution of electrical engineers (Vol. 114, pp. 775-780).

Stone, J. M., Gardiner, T. A., Teuben, P., Hawley, J. F., \& Simon, J. B. (2008). Athena: a new code for astrophysical mhd. The Astrophysical Journal Supplement Series, $178(1), 137$.

Stosic, N., Smith, I., \& Kovacevic, A. (2005). Screw compressors: mathematical modelling and performance calculation. Springer Science \& Business Media. 
Svensson, P., \& Kristiansen, U. R. (2002). Computational modelling and simulation of acoutic spaces. In Audio engineering society conference: 22nd international conference: Virtual, synthetic, and entertainment audio.

Tai, S., Puryear, C., \& Castagna, J. P. (2009). Local frequency as a direct hydrocarbon indicator. In Seg technical program expanded abstracts 2009 (pp. 2160-2164). Society of Exploration Geophysicists.

Taner, M. T., Koehler, F., \& Sheriff, R. (1979). Complex seismic trace analysis. Geophysics, 44(6), 1041-1063.

Tang, H., \& Sotiropoulos, F. (1999). A second-order godunov method for wave problems in coupled solid-water-gas systems. Journal of Computational Physics, 151(2), $790-815$.

Tang, T., Hededal, O., \& Cardiff, P. (2015). On finite volume method implementation of poro-elasto-plasticity soil model. International journal for numerical and analytical methods in geomechanics, 39(13), 1410-1430.

Thomée, V. (2001). From finite differences to finite elements a short history of numerical analysis of partial differential equations. In Numerical analysis: Historical developments in the 20th century (pp. 361-414). Elsevier.

Thorborg, J. (2001). Constitutive modelling in thermomechanical processes, using the control volume method on staggered grid.

Thorborg, J., \& Hattel, J. (2003). Thermo-elasto-plasticity in solidification processes using the control volume method on staggered grid. In Modelling of casting, welding and advanced solidification processes.

Tisato, N., \& Madonna, C. (2012). Attenuation at low seismic frequencies in partially saturated rocks: Measurements and description of a new apparatus. Journal of Applied Geophysics, 86, 44-53.

Titarev, V. A., \& Toro, E. F. (2004). Finite-volume weno schemes for three-dimensional conservation laws. Journal of Computational Physics, 201(1), 238-260.

Torlak, M. (2006). A finite-volume method for coupled numerical analysis of incompressible fluid flow and linear deformation of elastic structures. Techn. Univ., Arbeitsbereiche Schiffbau. 
Torlak, M., Muzaferija, S., \& Peric, M. (2002). Application of a finite volume method to the computation of interaction between thin linearly elastic structures and incompressible fluid flows. VDI BERICHTE, 1682, 205-220.

Toro, E. F. (2012). Godunov methods: Theory and applications. Springer Science \& Business Media.

Toro, E. F. (2013). Riemann solvers and numerical methods for fluid dynamics: a practical introduction. Springer Science \& Business Media.

Toro, E. F., Hidalgo, A., \& Dumbser, M. (2009). Force schemes on unstructured meshes i: Conservative hyperbolic systems. Journal of Computational Physics, 228(9), $3368-3389$.

Trangenstein, J. A. (1994). A second-order godunov algorithm for two-dimensional solid mechanics. Computational mechanics, 13(5), 343-359.

Trangenstein, J. A., \& Colella, P. (1991). A higher-order godunov method for modeling finite deformation in elastic-plastic solids. Communications on Pure and Applied Mathematics, 44(1), 41-100.

Trangenstein, J. A., \& Pember, R. B. (1992). Numerical algorithms for strong discontinuities in elastic_-plastic solids. Journal of Computational Physics, 103(1), 63-89.

Tukovic, Z., Cardiff, P., Karac, A., Jasak, H., \& Ivankovic, A. (2014). Openfoam library for fluid structure interaction. In 9th openfoam workshop (Vol. 2014).

Tuković, Ž., Ivanković, A., \& Karač, A. (2013). Finite-volume stress analysis in multimaterial linear elastic body. International journal for numerical methods in engineering, 93(4), 400-419.

Turkel, E. (1986). Accuracy of schemes with nonuniform meshes for compressible fluid flows. Applied Numerical Mathematics, 2(6), 529-550.

Turner, I. W., et al. (2003). A second order finite volume technique for simulating transport in anisotropic media. International Journal of Numerical Methods for Heat \& Fluid Flow.

Van Leer, B. (2006). Upwind and high-resolution methods for compressible flow: From donor cell to residual-distribution schemes. In 16th aiaa computational fluid $d y$ namics conference (p. 3559). 
Varga, R. S. (1962). Iterative analysis. Springer.

Vaz Jr, M., Muñoz-Rojas, P. A., \& Filippini, G. (2009). On the accuracy of nodal stress computation in plane elasticity using finite volumes and finite elements. Computers \& Structures, 87(17-18), 1044-1057.

Vázquez-Cendón, E., Hidalgo, A., Navarro, P. G., \& Cea, L. (2012). Numerical methods for hyperbolic equations. CRC Press.

Virieux, J. (1986). P-sv wave propagation in heterogeneous media: Velocity-stress finitedifference method. Geophysics, 51(4), 889-901.

Voller, V. R. (2009). Basic control volume finite element methods for fluids and solids (Vol. 1). World Scientific.

Vorländer, M. (2007). Auralization: fundamentals of acoustics, modelling, simulation, algorithms and acoustic virtual reality. Springer Science \& Business Media.

Vorländer, M. (2013). Computer simulations in room acoustics: Concepts and uncertainties. The Journal of the Acoustical Society of America, 133(3), 1203-1213.

Wang, L. X., \& Melnik, R. V. N. (2007). Finite volume analysis of nonlinear thermomechanical dynamics of shape memory alloys. Heat and mass transfer, 43(6), $535-546$.

Wang, R. (2012). Body fitted grids based fvm simulation of aluminum extrusion process. In Advanced materials research (Vol. 418, pp. 2102-2105).

Wang, R., \& Li, H. Z. (2011). Modeling of aluminum extrusion process using nonorthogonal block structured grids based fvm. In Advanced materials research (Vol. 189, pp. 1749-1752).

Webb, C. J. (2014). Parallel computation techniques for virtual acoustics and physical modelling synthesis.

White, L., \& Adcroft, A. (2008). A high-order finite volume remapping scheme for nonuniform grids: The piecewise quartic method (pqm). Journal of Computational Physics, 227(15), 7394-7422.

Wiedemair, W., Tuković, Ž., Jasak, H., Poulikakos, D., \& Kurtcuoglu, V. (2012). On ultrasound-induced microbubble oscillation in a capillary blood vessel and its implications for the blood-brain barrier. Physics in Medicine \& Biology, 57(4), 1019. 
Williams, A., Croft, T., \& Cross, M. (2002). Computational modelling of metal extrusion and forging processes. Journal of Materials Processing Technology, 125, 573-582.

Williams, A., Slone, A., Croft, T., \& Cross, M. (2010). A mixed eulerian-lagrangian method for modelling metal extrusion processes. Computer Methods in Applied Mechanics and Engineering, 199(33-36), 2123-2134.

Xing, Y., \& Shu, C.-W. (2006). High order well-balanced finite volume weno schemes and discontinuous galerkin methods for a class of hyperbolic systems with source terms. Journal of Computational Physics, 214(2), 567-598.

Xiong, X., He, Z., \& Huang, D. (2008). Seismic attenuation modeling of fluid-filled porous medium. In Seg technical program expanded abstracts 2008 (pp. 21522155). Society of Exploration Geophysicists.

Yee, K. (1966). Numerical solution of initial boundary value problems involving maxwell's equations in isotropic media. IEEE Transactions on antennas and propagation, 14(3), 302-307.

Zarrabi, K., \& Basu, A. (1999). An axisymmetric finite volume formulation for creep analysis. Journal of the Mechanical Behavior of Materials, 10(5-6), 325-340.

Zarrabi, K., \& Basu, A. (2000). A finite volume element formulation for solution of elastic axisymmetric pressurized components. International journal of pressure vessels and piping, 77(8), 479-484.

Zhang, C., Chen, H., Zhao, G., Zhang, L., \& Lou, S. (2016). Optimization of porthole extrusion dies with the developed algorithm based on finite volume method. The International Journal of Advanced Manufacturing Technology, 85(5-8), 1901-1913.

Zhang, H., Liu, M., Shi, Y., Yuen, D. A., Yan, Z., \& Liang, G. (2007). Toward an automated parallel computing environment for geosciences. Physics of the Earth and Planetary Interiors, 163(1-4), 2-22.

Zhao, H., Gao, J., \& Liu, F. (2014). Frequency-dependent reflection coefficients in diffusive-viscous mediafrequency-dependent reflection coefficients. Geophysics, 79(3), T143-T155.

Zhao, H., Gao, J., \& Peng, J. (2017). Extended reflectivity method for modelling the propagation of diffusive-viscous wave in dip-layered media. Geophysical Prospecting, 
$65,246-258$.

Zhao, H., Gao, J., Peng, J., \& Zhang, G. (2019). Modeling attenuation of diffusiveviscous wave using reflectivity method. Journal of Theoretical and Computational Acoustics, 27(03), 1850030.

Zhao, H., Gao, J., \& Zhao, J. (2014). Modeling the propagation of diffusive-viscous waves using flux-corrected transport-finite-difference method. IEEE Journal of Selected Topics in Applied Earth Observations and Remote Sensing, 7(3), 838-844.

Zheng, G., Kishk, A. A., Glisson, A. W., \& Yakovlev, A. B. (2006). Implementation of mur's absorbing boundaries with periodic structures to speed up the design process using finite-difference time-domain method. Progress In Electromagnetics Research, 58, 101-114.

Zhou, T., Li, Y., \& Shu, C.-W. (2001). Numerical comparison of weno finite volume and runge-kutta discontinuous galerkin methods. Journal of Scientific Computing, $16(2), 145-171$.

Zienkiewicz, O. C. (1977). The finite element method (mcgrawhill, london). Chap, 6, 96.

Zienkiewicz, O. C., \& Oñate, E. (1992). Finite elements versus finite volumes. is there really a choice? 